\title{
Obesity prevention using the Internet : development and evaluation of a video- and text-based version of a computer-tailored intervention
}

Citation for published version (APA):

Walthouwer, M. J. L. (2015). Obesity prevention using the Internet : development and evaluation of a video- and text-based version of a computer-tailored intervention. [Doctoral Thesis, Maastricht University]. Maastricht University. https://doi.org/10.26481/dis.20151022mw

Document status and date:

Published: 01/01/2015

DOI:

10.26481/dis.20151022mw

Document Version:

Publisher's PDF, also known as Version of record

Please check the document version of this publication:

- A submitted manuscript is the version of the article upon submission and before peer-review. There can be important differences between the submitted version and the official published version of record.

People interested in the research are advised to contact the author for the final version of the publication, or visit the DOI to the publisher's website.

- The final author version and the galley proof are versions of the publication after peer review.

- The final published version features the final layout of the paper including the volume, issue and page numbers.

Link to publication

\footnotetext{
General rights rights.

- You may freely distribute the URL identifying the publication in the public portal. please follow below link for the End User Agreement:

www.umlib.nl/taverne-license

Take down policy

If you believe that this document breaches copyright please contact us at:

repository@maastrichtuniversity.nl

providing details and we will investigate your claim.
}

Copyright and moral rights for the publications made accessible in the public portal are retained by the authors and/or other copyright owners and it is a condition of accessing publications that users recognise and abide by the legal requirements associated with these

- Users may download and print one copy of any publication from the public portal for the purpose of private study or research.

- You may not further distribute the material or use it for any profit-making activity or commercial gain

If the publication is distributed under the terms of Article $25 \mathrm{fa}$ of the Dutch Copyright Act, indicated by the "Taverne" license above, 


\section{Obesity prevention using the Internet}

Development and evaluation of a video- and text-based version of a computer-tailored intervention 


\section{Colophon}

Cover: Ridderprint BV, the Netherlands

Printing: Ridderprint BV, the Netherlands

(C) Michel Walthouwer, 2015

ISBN: 978-94-6299-149-1

The research presented in this dissertation was conducted at the School for Public Health and Primary Care (CAPHRI), Department of Health Promotion, Maastricht University. CAPHRI participates in the Netherlands School of Primary Care Research $(\mathrm{CaRe})$. The research presented in this dissertation was funded by the Netherlands Organization for Health Research and Development (ZonMw, grant number 200110001).

All rights are reserved. No part of this book may be reproduced or transmitted in any form or by any means, without the written permission from the author or, where appropriate, the publisher of the article. 


\title{
Obesity prevention using the Internet
}

Development and evaluation of a video- and text-based version of a computer-tailored intervention

\author{
Proefschrift \\ ter verkrijging van de graad van doctor aan de Universiteit Maastricht, \\ op gezag van de Rector Magnificus, prof. dr. L.L.G. Soete \\ volgens het besluit van het College van Decanen, \\ in het openbaar te verdedigen \\ op donderdag 22 oktober 2015 om 14:00 uur \\ door \\ Michel Jean Louis Walthouwer \\ geboren op 14 juli 1987 te Maastricht
}




\section{Promotores}

Prof. dr. Hein de Vries

Prof. dr. Lilian Lechner

\section{Copromotor}

Dr. Anke Oenema

\section{Beoordelingscommissie}

Prof. dr. Stef Kremers (voorzitter)

Dr. Catherine Bolman, Open Universiteit

Dr. Rik Crutzen

Prof. dr. Lisette van Gemert-Pijnen, Universiteit Twente

Prof. dr. Luc de Witte 


\section{Content}

\section{Chapter 1}

General introduction

\section{Part 1: Exploratory studies}

\section{Chapter 2}

Eating in moderation and the essential role of awareness: a Dutch longitudinal study identifying psychosocial predictors

\section{Chapter 3}

Are clusters of dietary patterns and cluster membership stable over time? Results of a longitudinal cluster analysis study

\section{Part 2: Intervention development and evaluation}

\section{Chapter 4}

Systematic development of a video and text version of a web-based computertailored obesity prevention intervention

\section{Chapter 5}

Are there differences in effects and appreciation between a video and text version of a web-based computer-tailored obesity prevention intervention?

\section{Chapter 6}

Use and effectiveness of a video and text version of a web-based computer-tailored obesity prevention intervention: does delivery format matter?

\section{Chapter 7}

A qualitative study on barriers and facilitators to the implementation of a web-based computer-tailored obesity prevention intervention by occupational health centers

\section{Chapter 8}

General discussion

Valorization addendum 



\section{CHAPTER 1}

General introduction 
Approximately 2 billion people worldwide are overweight or obese today, which is nearly $30 \%$ of the global population (Finucane et al., 2011; Stevens et al., 2012; WHO, 2015a, 2015b). These people have an increased risk of developing health problems, such as coronary heart disease, type 2 diabetes mellitus, and musculoskeletal disorders (Visscher, van Bakel, \& Zantinge, 2014; WHO, 2015b; Wyatt, Winters, \& Dubbert, 2006). Hence, there is a need for interventions that can contribute to the prevention of obesity in a costeffective manner. This dissertation aims to contribute to this requisite by examining the appreciation, use, and efficacy of a video and text version of a web-based computer-tailored obesity prevention intervention among lower and higher educated Dutch adults.

This chapter will first provide a detailed overview of the prevalence, consequences, and causes of overweight and obesity. Subsequently, the potential to prevent weight gain via small changes will be discussed, followed by an overview of the rationale for why the delivery of intervention content via videos may improve the effects of webbased computer-tailored obesity prevention interventions. The chapter will end with an overview of the aims and outline of the dissertation.

\subsection{Overweight and obesity}

Overweight and obesity have been defined as abnormal or excessive fat accumulation that can represent a risk to health (WHO, 2015a). The Body Mass Index (BMI) is the most used index to classify overweight and obesity in adults. An adult's BMI is calculated by dividing the body weight in kilograms by the square of the height in meters (WHO, 2015a). Based on expert committee recommendations, a person is considered to be overweight when the $\mathrm{BMI}$ is equal to or more than 25 , whereas a $\mathrm{BMI}$ of 30 or more indicates that a person is obese (WHO, 2015a; Wyatt et al., 2006).

Globally, the prevalence of obesity among adults has increased from $6.4 \%$ in 1980 to $13.0 \%$ in 2014 , while adult overweight increased from $24.6 \%$ to $39.0 \%$ during this period (Finucane et al., 2011; Stevens et al., 2012; WHO, 2015b). This means that the worldwide prevalence of overweight and obesity has more than doubled between 1980 and 2014 (WHO, 2015b). Currently, about 1.4 billion adults are overweight and over 600 million adults are obese (WHO, 2015b). The rise in BMI has further accelerated in the last decade and it is expected that by $203042 \%$ of the adult population worldwide will be obese (Finkelstein, Ruhm, \& Kosa, 2005). There further is an important socioeconomic gradient in overweight and obesity. In high-income countries in Europe and the Americas the prevalence of overweight and obesity is, for example, more than double that of low-income countries (WHO, 2015b). Within many high-income countries, this gradient is just the opposite and overweight and obesity rates are significantly higher among people with a low educational level (Devaux \& Sassi, 2011; McLaren, 2007; Roskam et al., 2010). In the Netherlands, for example, the prevalence of overweight is $64.4 \%$ among adults with a low educational level compared to $40.1 \%$ among adults with a high educational level. A similar pattern is seen for obesity with $23.6 \%$ of the people with a low educational level being 
obese compared to only $7.9 \%$ of those with a high educational level (Savelkoul \& Uiters, 2014). Hence, there is a need for weight management interventions that are effective and available for large numbers of people. In addition, these interventions should be in particular effective for the high-risk group of people with a low educational level.

Many studies have shown that overweight and obesity increase the risk of coronary heart disease, type 2 diabetes mellitus, ischemic stroke, and cancer of the breast, colon, prostate, kidney, endometrium, and gall bladder ( $\mathrm{Ng}$ et al., 2014; Visscher et al., 2014; WHO, 2015b; Wyatt et al., 2006). In addition, (severe) overweight can also lead to musculoskeletal disorders (e.g. osteoarthritis), respiratory problems, and infertility ( $\mathrm{Ng}$ et al., 2014; Visscher et al., 2014; Wyatt et al., 2006). Moreover, overweight and obese people have a significantly higher chance of developing mental disorders (e.g. depression or anxiety disorders) and experience stigmatization and discrimination compared to people with a healthy weight (Carr \& Friedman, 2005; Visscher et al., 2014; Wyatt et al., 2006). Worldwide, annually around 3.4 million adults die as a result of being overweight or obese (Ng et al., 2014). The life expectancy of overweight persons is about three years less compared to people without (severe) overweight (Visscher et al., 2014). In addition, overweight and obesity also have an adverse effect on the economy (Wyatt et al., 2006). For example, the annual medical costs for obese adults are $36 \%$ higher than the medical expenditures for normal-weight adults (Finkelstein et al., 2005). In addition to these direct medical costs, overweight and obesity also have high indirect costs related to, for example, decreased productivity, restricted activity, and absenteeism (CDC, 2012; Finkelstein et al., 2005; Neovius, Neovius, Kark, \& Rasmussen, 2012; Wyatt et al., 2006). Successful obesity prevention efforts, therefore, have the potential to result in substantial savings for health care and society (Finkelstein et al., 2012).

\subsection{Energy balance}

Overweight and obesity are the result of a long-term imbalance between energy intake and energy expenditure. Hence, to prevent weight gain it is important to achieve an energy balance between calories consumed and calories expended (Hall et al., 2012; WHO, 2013b). When there is a balance between energy intake and energy expenditure, the body weight will remain stable. However, when a person's energy intake is higher than the amount of energy that he or she expends, a positive energy balance will occur and the person will gain weight. Conversely, a negative energy balance means that the energy intake is lower than the energy expenditure and this will result in weight loss (Hill, Wyatt, \& Peters, 2012). Physical activity is the most influenceable source of energy expenditure, whereas energy intake is determined by dietary intake. Since these two energy-balance related behaviors are highly interrelated, strategies to address the obesity epidemic are likely to be most effective when both dietary intake and physical activity are taken into account (Hall et al., 2012; Hall et al., 2011; Hill, 2006; Hill, Wyatt, Reed, \& Peters, 2003; Sparling, Franklin, \& Hill, 2013). 


\subsection{Energy intake}

Weight gain is partially caused by excessive energy intake, which is the result of unhealthy dietary patterns characterized by a high consumption of non-core energy-dense foods and drinks such as fast food, snacks, and sugar-sweetened beverages (Ello-Martin, Ledikwe, \& Rolls, 2005; Grafenauer, Tapsell, Beck, \& Batterham, 2013; Kral, Roe, \& Rolls, 2004; Newby et al., 2003; Rolls, Roe, \& Meengs, 2006). In addition, the increase in body weight can also be explained by the increased consumption of large food portions (Ello-Martin et al., 2005; Gretebeck, Ferraro, Black, Holland, \& Gretebeck, 2012; Kral et al., 2004; Rolls et al., 2006; Rolls, Roe, \& Meengs, 2007). Many food products have increased in portion size during the last 20 years and this promotes the overconsumption of energy (Ello-Martin et al., 2005; Gretebeck et al., 2012; Steenhuis, Leeuwis, \& Vermeer, 2010; Steenhuis \& Vermeer, 2009). Moreover, it has been suggested that the increased eating frequency (i.e. number of times per day one eats) also contributes to excessive energy intake and an increased overweight and obesity prevalence (McCrory, Howarth, Roberts, \& Huang, 2011). It should further be noted that unhealthy dietary patterns and a high average daily energy intake are in particular more prevalent among persons with a low educational level (Darmon \& Drewnowski, 2008; Giskes, Avendaňo, Brug, \& Kunst, 2010; Konttinen, Sarlio-Lähteenkorva, Silventoinen, Männistö, \& Haukkala, 2013).

In conclusion, to prevent a positive energy balance and accordingly weight gain people should adopt a moderate eating pattern (Gretebeck et al., 2012; Hill, 2006; Rolls et al., 2006). This recently identified dietary pattern that has the potential to prevent weight gain includes the consumption of small portion sizes, limiting the frequency of eating occasions, reducing the intake of energy-dense foods, and increasing the consumption of low-energy-dense foods such as fruit and vegetables (Rolls et al., 2006; Swinburn, Caterson, Seidell, \& James, 2004). Yet, there currently is a lack of insight into the determinants of eating in moderation, which makes it very difficult to develop evidencebased interventions that can target this complex dietary behavior efficiently. In line with determinant studies into complex behaviors (Bogers, Brug, van Assema, \& Dagnelie, 2004; Brug, Conner, et al., 2005; Greene et al., 1999; Lechner, Bolman, \& Van Dijke, 2006; Ronda, van Assema, \& Brug, 2001), it has been hypothesized that lack of awareness may be a key factor hindering eating in moderation: people who incorrectly believe that they eat in moderation may not perceive a need to change and accordingly will have no motivation to change their behavior. One of the aims of this dissertation is identifying the psychosocial determinants of eating in moderation and examining the possible moderating role of awareness.

\subsection{Energy expenditure}

Another cause of the high worldwide overweight and obesity prevalence rates is decreased energy expenditure (Hill et al., 2012). Physical activity is at least as important as dietary intake in the etiology of weight gain (CDC, 2001; Dipietro, 1994). It is defined as any 
bodily movement produced by skeletal muscles that requires energy expenditure (WHO, 2015c). Worldwide at least $31 \%$ of individuals aged 15 and older (1.5 billion people) do not get sufficient physical activity (Dumith, Hallal, Reis, \& Kohl, 2011; Hallal et al., 2012). In particular having a low level of education has been associated with low physical activity levels (Droomers, Schrijvers, \& Mackenbach, 2001; Shaw \& Spokane, 2008). In the Netherlands, about half of the adult population does not meet the Dutch physical activity standard of at least half an hour of moderate- or vigorous-intensity physical activity on at least five days a week, but preferably every day (Wendel-Vos, 2014a, 2014b). Examples of moderate-intensity physical activity include brisk walking or cycling, whereas sports activities such as jogging, swimming, and playing football are examples of vigorousintensity physical activity.

Low physical activity levels are partly due to increased inactivity during leisure time and work (Tremblay, Colley, Saunders, Healy, \& Owen, 2010; WHO, 2015c). Occupational physical activity has, for example, decreased by 140 kilocalories per day since 1960 and currently $41.5 \%$ of adults spend 4 hours or more of sitting per day (Church et al., 2011; Hallal et al., 2012). This implies that people's energy expenditure can be increased by increasing the frequency and/or duration of physical activity. Twenty minutes of walking per day in addition to people's current physical activity level is, for example, sufficient to prevent weight gain (Hill, 2009).

\subsection{Weight gain prevention}

Prevention of weight gain is assumed to be the best way to counteract the worldwide rise in body weight since treatments and pharmacotherapies to attain weight loss frequently have proven to be ineffective (Hill, 2009; Hill et al., 2012). Treatments are able to achieve weight loss, but weight regain over time is very high (Hill, 2009; Weiss, Galuska, Khan, Gillespie, \& Serdula, 2007). In general, people are not able to achieve and sustain the large behavioral changes that are required for long-term weight loss maintenance (Hill et al., 2012). It therefore has been suggested that it may be more feasible for overweight people to try to prevent further weight gain or achieve modest weight loss (Hill et al., 2012). In addition, people with a healthy weight should aim to prevent future weight gain as well (Hill et al., 2012). Prevention of weight gain or achieving modest weight loss is more likely to be successful than following strict treatments since the human body shows a stronger opposition to large weight loss than weight maintenance. It is, therefore, easier to maintain weight than to produce large and sustained reductions in body weight (Hill et al., 2012).

The prevention of weight gain can already be achieved with small and relatively easy to achieve behavior changes of approximately 100 kilocalories per day (Hill, 2009; Hill et al., 2012). This is equal to, for example, walking 2,000 more steps (i.e. about 20 minutes) per day (Hill, 2009). For dietary intake, a reduction of 100 kilocalories can be achieved easily by the consumption of products with a lower energy density or consuming smaller portion sizes. Drinking a diet soda instead of a regular soda is, for example, already 
sufficient to prevent weight gain (Hill, 2009). To achieve modest weight loss (i.e. about 5 kilograms per year), small daily changes of about 200 kilocalories in dietary or physical activity behavior would be required (Hill, 2009). Research has shown that interventions advocating small changes can be effective in increasing physical activity and reducing energy intake as well as in the prevention and reduction of weight gain (Hill, 2009; Lutes et al., 2008; Rodearmel et al., 2007; Stroebele et al., 2009). Small changes seem to be more feasible to achieve and maintain than large changes (Hill, 2009). Moreover, achieving small lifestyle changes can lead to an increase in self-efficacy, which can accordingly stimulate people to make additional small changes (Hill, 2009). Small reductions in energy density and portion size can further be made without increased feelings of hunger (Hill, 2009). Hence, interventions aimed at weight gain prevention should stimulate and help people make small changes in dietary intake and physical activity (Hill, 2009). However, despite the potential efficacy of the small changes approach to prevent weight gain or achieve modest weight loss in a relatively easy way, thus far only few interventions are framed around this approach. Hence, more intervention studies are required in order to improve and broaden the scientific evidence about the usefulness and efficacy of the small changes approach. Future research should further also assess if it is more effective to achieve small changes by targeting interventions at whole dietary patterns or at separate (energy-dense) food products. Currently much is unknown about this, while this insight is of crucial importance for the potential efficacy of weight management interventions. Hence, one of the aims of this dissertation is to provide insight into whether or not it is efficient to target interventions at clusters of dietary patterns.

\subsection{Computer-tailoring}

Weight management interventions should take into account that obesity is a complex and multifactorial condition that affects many people (Malterud \& Tonstad, 2009). These interventions cannot rely on a one-size-fits-all approach because people will have a different preference about how they want to manage their body weight (Malterud \& Tonstad, 2009). The causes of obesity are different for each person and consequently some people will try to improve their dietary intake, while others will focus on their physical activity level. This implies that weight management interventions need to be highly individualized. In addition, because many people are overweight, these interventions should also have the possibility to reach many people in an efficacious yet cost-effective manner (Marcus, Nigg, Riebe, \& Forsyth, 2000). It has been suggested that web-based computertailored interventions meet all these requirements (Brug, Campbell, \& van Assema, 1999; van Genugten, van Empelen, Flink, \& Oenema, 2010). Web-based computer-tailored interventions aim to improve people's health behavior by providing tailored information and feedback via the Internet about, for example, their dietary intake, physical activity level, and motivational beliefs. To be able to provide tailored feedback, an online questionnaire should be completed consisting of questions about, for example, individual 
behavior (e.g. dietary intake or physical activity level), socio-demographic variables (e.g. gender, age, or educational level), and psychosocial factors (e.g. attitude or self-efficacy). Answers to this questionnaire are analyzed and processed by a software package that links up answers to (a combination of) questions with a message library. Based on tailoring algorithms, a message will be selected from this library that fits with the specific answers and characteristics of the respondent. In this way, individualized intervention material is created and the process of personal counseling is partially mimicked (Brug et al., 1999; Brug, Oenema, \& Campbell, 2003; de Vries \& Brug, 1999; Kreuter \& Skinner, 2000; van Gemert-Pijnen, Peters, \& Ossebaard, 2013). It has been suggested that individualized messages enhance cognitive preconditions for information processing and acceptance, such as attention and self-referential thinking (Hawkins, Kreuter, Resnicow, Fishbein, \& Dijkstra, 2008; Ruiter, Kessels, Jansma, \& Brug, 2006). Research has, for example, shown that tailored information is more likely to be read and remembered than non-tailored information (Campbell et al., 1994; Skinner, Strecher, \& Hospers, 1994). Tailored messages further contain less redundant information and are experienced as more personally relevant (Brug et al., 2003). Because the information processing capacity of people is limited, especially among those with a low educational level, it is important to provide only relevant information (Brug et al., 1999).

In the Netherlands, $94.0 \%$ of the population has access to the Internet and people are increasingly using the Internet as source for searching health-related information (Internet World Stats, 2014; The World Bank, 2015). Although access to the Internet is a little lower among people with a low educational level (i.e. 90\%) compared to higher educated people (i.e. 99\%), this still is sufficient to reach large numbers of people (van Deursen \& van Dijk, 2012). Hence, in principle web-based computer-tailored interventions have the potential to fit with people's current lifestyle since Internet use has become a major activity in daily life (Internet World Stats, 2014; Moen et al., 2012; The World Bank, 2015). Another significant advantage of these interventions is that they can reach many people at relatively low costs. Once a web-based computer-tailored intervention is developed, it can be disseminated easily via the Internet among a large target group, while personal counseling is expensive and time-consuming and therefore inappropriate for reaching large numbers of people (Brug et al., 1999; Brug et al., 2003; Marcus et al., 2000). By using computer-tailored interventions large segments of the population can thus receive information that is highly individualized (de Vries \& Brug, 1999). In addition, web-based computer-tailored interventions can be used in privacy (i.e. anonymous) and at an own preferred time, which makes them convenient in use (Bennett \& Glasgow, 2009). Hence, web-based computer-tailored interventions can be a promising approach for improving complex behaviors such as dietary intake and physical activity and eventually preventing weight gain. Yet, evidence suggests that the impact of web-based computer-tailored weight management interventions thus far has been limited (Arem \& Irwin, 2011; Bennett \& Glasgow, 2009; Broekhuizen, Kroeze, van Poppel, Oenema, \& Brug, 2012; de Nooijer et 
al., 2005; Enwald \& Huotari, 2010; Eysenbach, 2005; Krebs, Prochaska, \& Rossi, 2010; Marcus et al., 2000; Neville, O'Hara, \& Milat, 2009a, 2009b; Portnoy, Scott-Sheldon, Johnson, \& Carey, 2008; van Genugten, van Empelen, Boon, et al., 2012; Vandelanotte, Spathonis, Eakin, \& Owen, 2007; Wangberg, Bergmo, \& Johnsen, 2008).

\subsection{Intervention impact}

The impact of an intervention is determined by the number of people recruited to participate, actual use of the intervention, and the efficacy of the intervention itself (Marcus et al., 2000).

Unfortunately, despite the high level of reach and accessibility of the Internet, many studies evaluating web-based computer-tailored interventions have reported problems with recruiting a large and representative population (Bennett \& Glasgow, 2009; Brouwer et al., 2009; Eysenbach, 2005; Glasgow et al., 2007; Portnoy et al., 2008; Schneider et al., 2011). In particular important target groups, such as people with a low educational level, seem to be difficult to recruit (Schneider, Schulz, Pouwels, de Vries, \& van Osch, 2013). This indicates that there is huge potential for improving the recruitment rates for web-based computer-tailored interventions (Bennett \& Glasgow, 2009). Future research, for example, needs to examine which factors should be targeted in implementation strategies in order to improve the recruitment of users for these interventions (Bennett \& Glasgow, 2009; Schneider et al., 2013; Schneider et al., 2011; Spittaels \& de Bourdeaudhuij, 2006).

Moreover, many studies have also reported problems regarding the use of webbased computer-tailored interventions (Crutzen, 2009; de Nooijer et al., 2005; Glasgow, Vogt, \& Boles, 1999; Spittaels, de Bourdeaudhuij, Brug, \& Vandelanotte, 2007; Spittaels, de Bourdeaudhuij, \& Vandelanotte, 2007; Vandelanotte et al., 2007). For an intervention to have any impact the target population must be exposed to and involved in the intervention, preferably over an extended period of time (Brug et al., 2003). Unfortunately, first as well as sustained use of web-based computer-tailored interventions generally is low (Bennett \& Glasgow, 2009; Spittaels, de Bourdeaudhuij, \& Vandelanotte, 2007; Vandelanotte et al., 2007; Wang, Schwartz, Luta, Maxwell, \& Mandelblatt, 2012). Despite the fact that most participants are volunteers and thus must have some motivation to use the intervention, attrition rates of $75 \%$ are not uncommon (Bennett \& Glasgow, 2009; Spittaels, de Bourdeaudhuij, \& Vandelanotte, 2007; J. Wang et al., 2012; Wangberg et al., 2008). Discontinued use causes that participants will not be exposed to all essential intervention content. Moreover, web-based computer-tailored interventions have in particular problems with engaging and retaining people with a low educational level (Schneider, van Osch, Schulz, Kremers, \& de Vries, 2012). Hence, the efficacy of future web-based computertailored interventions can potentially be improved by gaining more insight into how (re)visits to these interventions can be stimulated (Arem \& Irwin, 2011; Bennett \& Glasgow, 2009; Eysenbach, 2005; Spittaels, de Bourdeaudhuij, \& Vandelanotte, 2007). 
Finally, the evidence for the effects of web-based computer-tailored interventions targeting weight management and energy balance-related behaviors is inconsistent. While some studies have reported positive outcomes, others did not (Arem \& Irwin, 2011; Broekhuizen et al., 2012; Enwald \& Huotari, 2010; Krebs et al., 2010; Lustria, Cortese, Noar, \& Glueckauf, 2009; Neville et al., 2009a; Spittaels \& de Bourdeaudhuij, 2006; van Genugten, van Empelen, Boon, et al., 2012; Vandelanotte et al., 2007). These interventions have proven to be effective in achieving dietary behavioral change, but the results for physical activity and BMI are mixed (Arem \& Irwin, 2011; Broekhuizen et al., 2012; Enwald \& Huotari, 2010; Neville et al., 2009b). Whereas some studies have demonstrated positive effects on physical activity, many studies have not found significant effects on this behavior (Broekhuizen et al., 2012; Enwald \& Huotari, 2010; Neville et al., 2009b; van Stralen, de Vries, Mudde, Bolman, \& Lechner, 2011; Vandelanotte et al., 2007). Moreover, the magnitude of the effects of these interventions generally is small and less than found for individual or group treatment approaches (Arem \& Irwin, 2011; Broekhuizen et al., 2012). Effects are further mostly found within the first six months of the intervention among small study samples and there is little evidence that effects are generalizable and sustained on the long-term (Bennett \& Glasgow, 2009; Broekhuizen et al., 2012; Enwald \& Huotari, 2010; Neville et al., 2009a; Vandelanotte et al., 2007). Moreover, most interventions have been targeted at physical activity or dietary intake without an explicit focus on weight management. In addition, the effectiveness of weight management interventions generally is lower among people with a low educational level (Magnée et al., 2013). Hence, future research is required in order to examine how the efficacy of web-based computer-tailored weight management interventions can be improved.

In conclusion, to increase the impact of web-based computer-tailored weight management interventions, future research needs to develop and examine strategies that can improve the recruitment, use, and efficacy of these interventions, particularly among people with a low educational level (Arem \& Irwin, 2011; Bennett \& Glasgow, 2009; Magnée et al., 2013; Neville et al., 2009b; Schneider et al., 2011; Vandelanotte et al., 2007).

\subsection{Improving the impact of web-based computer-tailored interventions through video delivery}

Most web-based computer-tailored weight management interventions have merely consisted of plain text-based information and this can possibly explain the limited impact of these interventions. Tailoring is an effective communication technique, but the way in which tailored messages are communicated may be even more important for achieving a significant impact (Brouwer et al., 2011; Wilson et al., 2010). If the delivery format does not fit with the receiver's preferences, the chance is high that a person will stop using an intervention or not start with it at all. On the contrary, receiving information via an appropriate and suitable delivery format may improve participation, reduce attrition, and increase effectiveness of an intervention (Floyd \& Moyer, 2010; King, 2000; King et al., 
2005; Marcus, Owen, Forsyth, Cavill, \& Fridinger, 1998; Vandelanotte, Duncan, Plotnikoff, \& Mummery, 2012). It has been hypothesized that the delivery of intervention content via videos has the potential to solve the previously mentioned problems with the impact of web-based computer-tailored weight management interventions.

The use of videos as delivery format is an innovative strategy that may fit better with people's current preferences. In videos, text-based information can be read out loud by one or more actors, whereas it is also possible to actually demonstrate skills or share learning experiences. Hence, videos can deliver intervention content livelier and more interactive. Accordingly, the use of videos may improve the appreciation of interventions and consequently lead to more intervention use (Alley et al., 2014; Lee, 2011; Marcus et al., 1998; Stanczyk, Bolman, Muris, \& de Vries, 2011; Vandelanotte \& Mummery, 2011; Wilson et al., 2010). It has further also been hypothesized that the use of videos to deliver intervention content is in particular suitable for persons with a low educational level since these people generally have lower literacy skills and more difficulties with the translation of abstract text into concrete actions (Gerber et al., 2005; Jacobson et al., 1999; Kandula et al., 2009; Meade, 1996; Paasche-Orlow, Parker, Gazmararian, Nielsen-Bohlman, \& Rudd, 2005; Stanczyk et al., 2011). The fact that people with a low educational level generally are less text-oriented can possibly also explain why the impact of many previous text-driven web-based computer-tailored interventions has been lower among these people. Information processing among people with a low educational level might be more influenced by videos since this activates both visual and verbal channels in the human mind (Campbell, Honess-Morreale, Farrell, Carbone, \& Brasure, 1999; Danaher, Jazdzewski, McKay, \& Hudson, 2005; Houts, Doak, Doak, \& Loscalzo, 2006; Mayer \& Massa, 2003; Petty \& Cacioppo, 1986a). It is assumed that videos reduce the cognitive effort needed to process information, since abstract text does not have to be translated into concrete action. This will free resources to process the main message and potentially lead to a better understanding (Salomon, 1984; Salomon \& Gardner, 1986; Sweller, 1994). Recent studies have further shown that video-tailored feedback can lead to greater attention and better appreciation compared to text-tailored feedback (Alley et al., 2014). Moreover, when people receive intervention content via videos they are more apt to be active in decision making processes (Krouse, 2001). In addition, previous research has shown that participants spend significantly more time with a video version of a web-based computertailored physical activity intervention compared to a similar text intervention (Alley et al., 2014; Soetens, Vandelanotte, de Vries, \& Mummery, 2014). More time spend with an intervention generally equals more exposure to intervention content and this makes it more likely that positive health behavior changes will be achieved (Frenn et al., 2005; Soetens et al., 2014). Several studies have also demonstrated that videos can be (cost-)effective in improving psychosocial beliefs as well as various health behaviors (Armstrong, Idriss, \& Kim, 2011; Frenn et al., 2005; Krawczyk et al., 2012; Krouse, 2001; Stanczyk, Bolman, et al., 2014; Stanczyk, Smit, et al., 2014; Tuong, Larsen, \& Armstrong, 2014; J. Wang et al., 
2012). The main aim of this dissertation is to examine the appreciation, use, and efficacy of a video and text version of a web-based computer-tailored obesity prevention intervention among lower and higher educated Dutch adults with a healthy weight or limited overweight. It is hypothesized that the video version will have the best effects, in particular among people with a low educational level.

\subsection{Potential working mechanisms of tailored videos}

Various theories of persuasion, including the Elaboration Likelihood Model (ELM) (Petty $\&$ Cacioppo, 1986b) and the Communication-Persuasion Model (CPM) (McGuire, 1981), provide support for the hypothesis that the use of a better fitting information delivery format (i.e. videos) can potentially improve the impact of web-based computer-tailored interventions.

According to the ELM, persuasion occurs along an elaboration likelihood continuum ranging from central to peripheral information processing (Braverman, 2008; Petty \& Cacioppo, 1986b). Central route processing occurs when people are relatively thoughtful in their consideration of the message. On the contrary, when people are little thoughtful in their consideration peripheral route processing will occur. People then rely on simple cues available in the persuasion context, such as a rule of thumb or number of arguments, rather than evaluating the message on its content. Information that is processed via the central route will have a more long lasting persuasive effect on the receiver, result in stronger attitudes, and is more predictive of behavior (Petty \& Cacioppo, 1986a). Via the peripheral route only part of the information will be processed and it is less likely that longterm changes will be achieved. Hence, it is important that interventions stimulate central route processing. Motivation and ability are two important factors that influence the route of information processing. Central route processing can, for example, be stimulated by matching the delivery format of information to an individual's personal characteristics since this will increase a recipient's ability and motivation to process information (Tam \& Ho, 2005). As it has been hypothesized that videos are a better fitting information delivery format for persons with a low educational level in particular, interventions that use this mode of delivery are more likely to stimulate central route processing (Braverman, 2008; Petty \& Cacioppo, 1986b).

The CPM is a step-based model of persuasion, which assumes that people need to transit through several steps or phases before being persuaded. The model consists of a matrix of inputs and outputs applied to persuasion (McGuire, 1981, 2000). The inputs are divided into five different aspects of the persuasive message that together influence its success, including source, message, channel, receiver, and context aspects. The model also makes a distinction between twelve different outputs, which are considered to be the consecutive stages of persuasion. The model, for example, assumes that a persuasive message should first lead to attention and comprehension before a certain behavior will be elicited. According to the model, the chance of positive outputs is higher when the input 
aspects of the persuasive attempt are dealt with satisfactory. Based on the assumptions of the CPM, it can therefore also be reasoned that the use of videos as delivery format (i.e. channel) will yield better effects (i.e. outcomes) as it fits more adequately with the characteristics of the receiver (i.e. particularly people with a low educational level).

\subsection{Theories of behavior change}

To have any impact, an intervention should also target the most important psychosocial factors associated with the behavior of interest. Many theories and models assume that health behaviors can be predicted and changed by understanding and intervening on persons' psychosocial beliefs. The most familiar ones are the Social Learning Theory (Bandura, 1997), the Health Belief Model (Janz \& Becker, 1984), the Protection Motivation Theory (Rogers \& Prentice-Dunn, 1997), the Theory of Planned Behavior (Ajzen, 1991), and the Transtheoretical Model (Prochaska \& Velicer, 1997). In particular the I-Change Model (ICM) (de Vries et al., 2003), which is an integration of these theories, and theories of self-regulation (Leventhal, Leventhal, \& Contrada, 1998; Zimmerman, 2000) are considered to be appropriate theoretical frameworks for understanding and changing complex behaviors such as dietary intake and physical activity.

Self-regulation can be defined as systematic efforts to direct thoughts, feelings, and actions toward the attainment of one's goals (Leventhal et al., 1998; Zimmerman, 2000). The essential elements of achieving behavior change have been represented in various self-regulation theories using different labels. In general, self-regulation theories assume that it is essential that people first become aware of their own health risk behavior. Next, a behavior change goal should be set followed by the selection and implementation of strategies to achieve the specified goal. Self-regulation theories further assume that it is essential that people monitor their goal progress and adjust strategies to ensure success by, for example, coping with barriers to one's goal. Eventually, people have to evaluate whether or not the implemented strategies have resulted in a successful behavior change and achievement of their goal. This evaluation can result in new goals and accordingly a repetition of the previous self-regulation stages, which is indicated with a feedback loop in many self-regulation theories (Leventhal et al., 1998; Zimmerman, 2000).

In line with self-regulation theories, the ICM demonstrates that people go through three different phases before a successful behavior change will be achieved: a premotivational, motivational, and post-motivational phase (de Vries et al., 2003). The premotivational phase consists of three factors: awareness of engaging in the risk behavior, knowledge, and risk perception (i.e. beliefs about the susceptibility to and severity of developing a certain disease). These factors subsequently influence three motivational factors, including attitude, self-efficacy, and social influence (i.e. modelling, social support, and social norm). Together, these factors determine a person's intention to perform a certain health behavior. In the post-motivational phase, this intention is translated into actual behavior by planning specific actions, skills development, and reducing potential 
barriers. The ICM further demonstrates that predisposing factors (i.e. behavioral, demographic, psychological, and biological factors) as well as environmental factors have an influence during all three phases. In relation to behavior change, the ICM also highlights the importance of various information factors when selecting intervention materials and behavior change techniques (BCT's), including personal, source, channel, and message factors (de Vries et al., 2003).

Because of the assumed usefulness of self-regulation models and the ICM to improve complex behaviors such as dietary intake and physical activity, these theories were used as theoretical underpinning of the web-based computer-tailored obesity prevention intervention.

\subsection{Aim and outline of dissertation}

The main aim of this dissertation is to examine the appreciation, use, and efficacy of a video and text version of a web-based computer-tailored obesity prevention intervention in comparison to a waiting list control condition on dietary intake, physical activity level, and BMI among lower and higher educated Dutch adults with a healthy weight or limited overweight. It is hypothesized that the video version will result in better effects among people with a low educational level in particular. The dissertation is divided into two parts, which both consist of several studies and research questions.

The aim of the first part is to describe the results of two exploratory studies that can be used as input for the development of web-based computer-tailored weight management interventions. The main research question of this part is: How can eating in moderation be stimulated in weight management interventions? This part consists of two chapters. Chapter 2 describes the results of a longitudinal study that identified the psychosocial determinants of eating in moderation. Chapter 3 presents the results of a longitudinal study that examined whether or not it is efficient to target interventions at clusters of dietary patterns.

The second part of this dissertation concerns the development and evaluation of the video and text version of the web-based computer-tailored intervention that aims to achieve small changes in dietary intake and physical activity in order to prevent weight gain (i.e. achieve weight maintenance or modest weight loss) among Dutch adults. The main research question of this part is: What are the effects of a video and text version of a webbased computer-tailored obesity prevention intervention at 6-month follow-up and do the effects differ per educational level? This part of the dissertation consists of four chapters. Chapter 4 includes a detailed description of the development process and content of the intervention. Chapter 5 describes the results of a randomized controlled trial (RCT) examining the efficacy and appreciation of the video and text version of the intervention at 6-month follow-up. Chapter 6 is based on the same RCT and provides detailed insight into the use of the two intervention versions and the influence of intervention use on its efficacy. Chapter 7 describes the results of a qualitative study into the barriers and facilitators to the 
adoption and particularly the implementation of the intervention by Dutch occupational health centers.

Finally, in chapter 8 the main findings of all previous chapters are discussed, including methodological considerations and recommendations for practice and future research. 


\section{PART 1}

Exploratory studies 


\section{CHAPTER 2}

Eating in moderation and the essential role of awareness: a Dutch longitudinal study identifying psychosocial predictors

Published as: Walthouwer, M., Oenema, A., Candel, M., Lechner, L., \& de Vries, H. (2015). Eating in moderation and the essential role of awareness. A Dutch longitudinal study identifying psychosocial predictors. Appetite, 87, 152-159. 


\begin{abstract}
Background: Eating in moderation, i.e. the attempt to monitor and limit the intake of energy-dense foods, is a promising strategy in the prevention of weight gain. The purpose of this study was to examine which psychosocial factors derived from the I-Change Model (ICM) were associated with eating in moderation, and whether these factors differed between adults with a correct (aware) or incorrect (unaware) perception of their dietary behavior.
\end{abstract}

Methods: This study used a longitudinal design with measurements at baseline $(\mathrm{N}=483)$ and 6-month follow-up ( $\mathrm{N}=379)$. Eating in moderation was defined as the average daily energy intake from energy-dense food products and was measured by a validated food frequency questionnaire. Linear regression analyses were used to assess the associations between the ICM factors and eating in moderation. The moderating role of awareness was examined by including interactions between awareness and the ICM factors in the regression analyses using the pick-a-point approach to further examine the associations for aware and unaware participants.

Results: Participants who were aware of their dietary behavior had a significantly lower average daily energy intake compared to those who were unaware. Eating in moderation was predicted by awareness, risk perception, social influence, and intention. Among the aware participants, eating in moderation was predicted by risk perception, attitude, social influence, and intention. Among the unaware participants, only risk perception and selfefficacy were significantly associated with eating in moderation.

Conclusions: Our findings show that psychosocial factors may only predict eating in moderation when people are aware of their risk behavior. Therefore, interventions aimed at promoting complex behaviors, such as eating in moderation, should first focus on improving individuals' awareness of their risk behavior before targeting motivational factors.

Keywords: eating in moderation, awareness, I-Change Model, obesity, determinants 


\section{Introduction}

In recent decades, the prevalence of overweight and obesity has increased dramatically (WHO, 2013a). In 2008, more than 1.4 billion adults worldwide were overweight, and of those, 500 million were obese (WHO, 2013a). In the Netherlands in 2012, about $35 \%$ of the adult population was overweight and 13\% were obese (Van den Brink \& Savelkoul, 2013). Overweight and obesity are caused by an imbalance between energy intake and energy expenditure (NHLBI, 2012; Van den Brink \& Savelkoul, 2013). The consumption of energy-dense foods in combination with the increased consumption of large portion sizes of especially energy-dense foods are an important cause of this excessive energy intake (ElloMartin et al., 2005; Hill, Wyatt, \& Melanson, 2000; Rolls et al., 2007; Steenhuis et al., 2010; Swinburn et al., 2004). Moreover, several studies have shown that the increase in eating frequency may be an important contributor as well (McCrory et al., 2011).

Eating in moderation is the reverse of consuming too much energy-dense food and too many large portions. It can be defined as the attempt to monitor and limit the intake of energy-dense foods in order to maintain or achieve a certain body weight (Haines, SiegaRiz, \& Popkin, 1999). This includes, for example, eating small portion sizes, limiting eating frequency, and eating low-energy-dense foods. A positive effect of eating in moderation is that it does not require following a strict diet (Rolls, 2009). Adopting a moderate eating pattern can result in sustained decreases in energy intake without increased feelings of hunger (Kral et al., 2004; Rolls et al., 2006). Hence, to prevent weight gain, adoption of a moderate eating pattern could be a promising strategy to promote in interventions aimed at the prevention of weight gain.

In order to be able to promote eating in moderation through interventions, a clear understanding of the most important determinants of eating in moderation is required (Bartholomew, Parcel, Kok, Gottlieb, \& Fernandez, 2011). However, to date no studies have been carried out to identify the determinants of this behavior. Hence, the aim of this study was to identify which psychosocial factors of the I-Change Model (ICM) (de Vries et al., 2003) are associated with eating in moderation. The ICM (see Figure 1) firstly postulates that various distal factors, such as demographic and biological factors, have an indirect effect on behavior via socio-cognitive variables. The most distal socio-cognitive variables are the pre-motivational factors, including knowledge, cues to action, risk perception (i.e. beliefs about the susceptibility to and severity of developing a certain disease), and awareness of engaging in the risk behavior (de Vries et al., 2003). These factors subsequently influence various motivational factors such as attitude (pros and cons), self-efficacy, and social influence (modelling, social support, and social norm). These motivational factors together influence an individual's intention to perform a certain health behavior. Of all these variables, in particular, awareness has been found to be an important factor in the explanation of complex health behaviors such as physical activity and dietary intake (Adams \& White, 2005; Brug, Conner, et al., 2005; Kloek, van Lenthe, van Nierop, \& Mackenbach, 2004; Ronda et al., 2001). It has been argued that an adequate awareness of 
one's own risk behavior is a precondition for becoming motivated to change complex behaviors (Bogers et al., 2004; Brug, Conner, et al., 2005; Greene et al., 1999; Lechner et al., 2006; Lechner, Brug, de Vries, van Assema, \& Mudde, 1998; Ronda et al., 2001; van Sluijs, Griffin, \& van Poppel, 2007; Weinstein, 1988). People who are aware of their (complex) risk behavior are more likely to consider change compared to people who are not aware that they are engaging in risk behavior (Bogers et al., 2004; Brug, van Assema, Kok, Lenderink, \& Glanz, 1994; Lechner et al., 2006; Lechner, Brug, \& de Vries, 1997; Ronda et al., 2001; van Sluijs et al., 2007; Weinstein, 1988). Awareness can thus be considered as a moderating variable within complex behaviors. We hypothesize that awareness of one's own risk behavior may also be an essential moderating factor for determinants associated with eating in moderation since this is a complex behavior as well. This would imply that people who incorrectly believe that they eat in moderation may not perceive a need to change and accordingly have no motivation to change their behavior. Correspondingly, we expect that the motivational factors of the ICM do not predict eating in moderation among respondents who are unaware of their behavior.

To examine this hypothesis, the aim of this study was to assess which psychosocial factors of the ICM are associated with eating in moderation and whether these factors differ between Dutch adults with a correct (aware) or incorrect (unaware) perception of their dietary behavior.

Figure 1. The I-Change Model

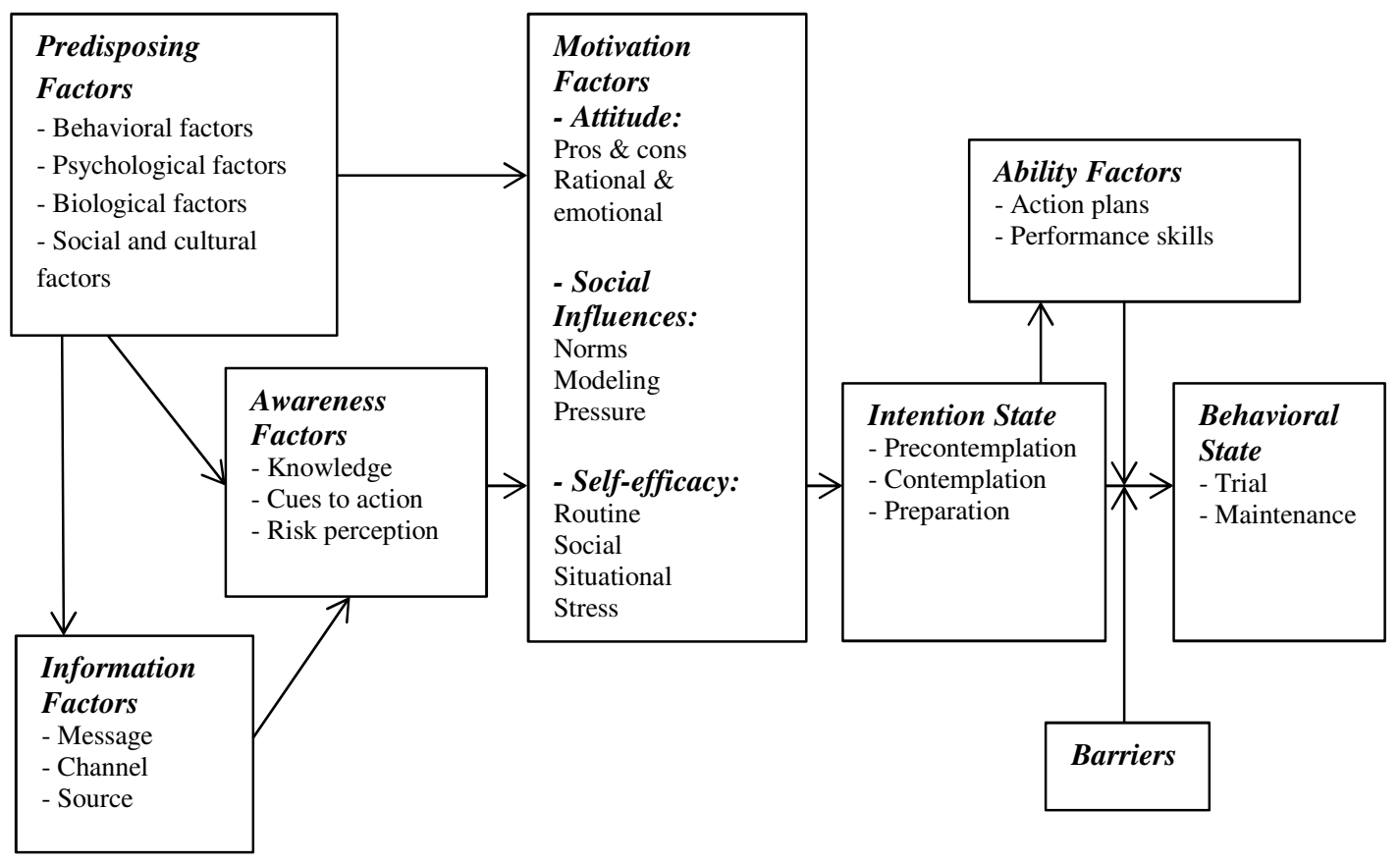




\section{Materials and methods}

\section{Study design and participants}

This study used a longitudinal design with a baseline measurement (T0) and a 6-month follow-up measurement (T1). Based on a power calculation suggested by Tabachnick and Fidell (2001), we needed to recruit at least 300 participants. For this purpose, members of an Internet research panel (Flycatcher, the Netherlands) were invited to participate in the study and to complete an online questionnaire. At baseline, 942 members of the online panel were approached, of which 483 participated (response=51\%) and 379 completed the follow-up questionnaire (response $=78 \%$ of those who started). Individuals were eligible to participate when they (a) were between 18 and 65 years old, (b) had a job, (c) had access to the Internet, and (d) were a member of the online panel.

\section{Questionnaire}

\section{Eating in moderation}

Eating in moderation was defined as the average daily intake of energy from energy-dense food products. A low score means that a participant eats in moderation, whereas a high score indicates that a participant is not eating in moderation. The average daily intake of energy-dense food products was assessed by means of a food frequency questionnaire consisting of 66 items, which was based on a validated questionnaire to assess fat intake (van Assema, Brug, Ronda, \& Steenhuis, 2001) and expanded with additional food categories (e.g. alcohol and sugar-sweetened beverages) in order to measure the intake of energy-dense food products that are common in the Dutch diet. The following food categories were included: dairy products, sandwiches and fillings, food eaten during dinner, sweet and savory snacks, hot and cold beverages, and alcohol. A high intake of foods from these categories has been associated with weight gain (Mozaffarian, Hao, Rimm, Willett, \& $\mathrm{Hu}, 2011$; Newby et al., 2003). For each food product, the frequency, i.e. 'On how many days do you generally eat/drink product x?', and quantity, i.e. 'On a day you eat/drink product $\mathrm{x}$, how much do you consume on average on a single day?', were assessed. For some food products, e.g. meat consumption, the portion size was assessed as well, i.e. 'On a day you eat/drink product $\mathrm{x}$, how many grams do you consume on average each time?' For some of the products, such as dairy, the type of product (low, medium or high fat) was also measured. A score for the average daily intake of the energy-dense products was calculated by multiplying the energy value of each product by the frequency and the quantity in which each food product was consumed. Further, we also made a dichotomous variable of eating in moderation using a median split. A score below the median was coded as eating in moderation, while a score above the median was coded as not eating in moderation. A complete overview of the measured food products, examples of questions and mean scores can be found in Table 1 . 


\section{Psychosocial determinants of eating in moderation}

The ICM was used as framework for including determinants concerning eating in moderation in the questionnaire. These determinants were only measured at baseline, with a total of 49 questions. All answering categories of the psychosocial variables consisted of a five-point Likert scale, ranging from 1 (=low) to 5 (=high). For each determinant except awareness, a scale was computed by calculating a mean score of all corresponding questions. The questionnaire also consisted of several explorative concepts which were not used for the present study, including anticipated regret, response efficacy, planning, and process evaluation questions about the questionnaire. Details on the assessed determinants, including number and examples of questions per determinant, answer categories, mean scores, and Cronbach's alphas, are presented in Table 1.

Awareness of eating in moderation was assessed by combining the dichotomous variable of eating in moderation with self-perceived eating in moderation. To assess the participants' own perceptions of eating in moderation, they were asked if they thought that they usually ate in moderation, i.e. 'I usually (never - always) eat in moderation'. This variable was dichotomized to 0 for not or not always eating in moderation and 1 for always eating in moderation. By combining this variable with the dichotomous variable concerning eating in moderation, four groups of participants could be distinguished: (1) participants who correctly indicated that they usually ate in moderation (aware); (2) participants who correctly indicated that they usually did not eat in moderation (aware); (3) participants who incorrectly indicated that they usually ate in moderation (unaware); and (4) participants who incorrectly indicated that they usually did not eat in moderation (unaware). For the analyses, these four groups were combined into a dichotomous variable, distinguishing between participants who were aware of their dietary behavior and participants who were unaware of their actual dietary behavior.

Risk perception was measured using seven questions for perceived severity, e.g. gaining weight is (not serious at all - very serious), and five questions for perceived susceptibility, e.g. 'If I do not eat in moderation, my body weight will rise'.

Participants' attitude toward eating in moderation was assessed by asking about their perception of ten pros, e.g. improved fitness, beneficial for health, maintaining weight, and ten cons, e.g. unhealthy, hunger, spoiling food. Social influence was measured with four questions for subjective norm, e.g. 'Most people who are important to me, think that eating in moderation is important', three questions for modelling, e.g. 'Most people who are important to me, eat in moderation', and six questions for social support, e.g. 'Most people who are important to me, encourage me to eat in moderation'. Self-efficacy was measured by two questions concerning the perceived difficulty to eat in moderation, e.g. 'I find it (very difficult - very easy) to eat in moderation'.

Finally, the participants' intention to eat in moderation was assessed by one question, i.e. 'Do you intend to eat in moderation within the next six months?' 


\section{Demographics}

The baseline questionnaire also assessed several self-reported demographic characteristics, including gender, age, educational level, household composition as well as height and body weight to calculate body mass index (BMI). Educational level, i.e. the highest level of education completed, was recoded into three categories: low (primary or basic vocational school), medium (secondary vocational school or high school), and high (higher vocational school or university) (Verweij, 2013). Household composition was dichotomized into living alone versus living together.

\section{Statistical analyses}

All statistical analyses were conducted using SPSS 19.0, applying a significance level of 0.05 for two-tailed tests. Descriptive statistics and frequencies were used to describe the study population. A logistic regression analysis was performed to examine possible selective attrition of participants between baseline and follow-up regarding demographics. Descriptive statistics, independent samples t-tests, and crosstab analyses were performed to describe the aware and unaware groups and to examine differences in demographic characteristics between these two subgroups. To identify determinants of eating in moderation, multiple linear regression analysis was performed by regressing the variables of the ICM on eating in moderation. To examine if level of awareness moderated the associations between the ICM variables and eating in moderation, interactions between awareness and ICM variables were included, which were further examined using the picka-point approach of Hayes and Matthes (2009) for probing interactions. The latter was done by performing two separate regression analyses with interaction terms between awareness and the ICM variables in the model and using a different reference category for awareness. In one analysis, awareness was categorized as 1 , while unawareness was categorized as 0 . In the second analysis, awareness was categorized as 0 and unawareness as 1 . By changing the coding, we were able to further examine the interaction terms with the full sample and get separate regression coefficients for each of the determinants according to the ICM for both awareness levels. In all regression analyses, the independent variables were entered in five consecutive steps as required by the theoretical model (ICM) that underpinned our study: (1) demographic factors, (2) pre-motivational factors, (3) motivational factors, (4) intention, and (5) eating in moderation measured at baseline, which was entered as a last step to examine its additional value. 
Table 1. Overview of number and examples of questions, answering categories, mean scores and Cronbach's alphas

\begin{tabular}{|c|c|c|c|c|}
\hline Concept & $\begin{array}{c}\text { Number of } \\
\text { questions }\end{array}$ & Examples of questions, answering options, and range & Mean (SD) & $\begin{array}{c}\text { Cronbach's } \\
\text { alpha }\end{array}$ \\
\hline \multicolumn{5}{|l|}{ Behavior } \\
\hline $\begin{array}{l}\text { Eating in moderation (i.e. average } \\
\text { daily energy intake from energy- }\end{array}$ & 66 & $\begin{array}{l}\text { - Frequency: On how many days do you generally eat/drink } \\
\text { product } x ?^{d}\end{array}$ & $906.0(393.3)^{\mathrm{b}}$ & - \\
\hline dense food products) & & $\begin{array}{l}\text { - Quantity: On a day you eat/drink product } \mathrm{x} \text {, how much do you } \\
\text { consume on average on a single day? } \\
\text { - Portion size: On a day you eat/drink product } \mathrm{x} \text {, how many grams } \\
\text { do you consume on average each time? } \\
\text { - Type of product: On a day you eat/drink product } \mathrm{x} \text {, what kind of } \\
\text { product do you usually consume? }\end{array}$ & & \\
\hline Dairy products & 13 & $\begin{array}{l}\text { Products: milk, chocolate milk, yogurt, sugar-sweetened dairy } \\
\text { beverages, pudding }\end{array}$ & $108.2(87.8)^{\mathrm{b}}$ & - \\
\hline Sandwiches and fillings & 13 & Products: bread, cheese, meat, chocolate spread, butter & $247.4(162.4)^{\mathrm{b}}$ & - \\
\hline Food eaten during dinner & 10 & Products: sausage, minced meat, bacon, cheese, gravy & $95.7(77.4)^{b}$ & - \\
\hline Sweet and savory snacks & 21 & $\begin{array}{l}\text { Products: fried snacks, peanuts, potato chips, cheese, cake, } \\
\text { chocolate, candy bars, biscuits, cookies }\end{array}$ & $262.0(214.2)^{\mathrm{b}}$ & - \\
\hline Hot and cold beverages & 5 & Products: coffee, tea, sugar-sweetened drinks & $140.8(146.9)^{\mathrm{b}}$ & - \\
\hline Alcohol & 4 & Products: beer, wine, spirits & $83.9(117.5)^{b}$ & - \\
\hline \multicolumn{5}{|l|}{ Determinants } \\
\hline Awareness of eating in moderation ${ }^{a}$ & 1 & $\begin{array}{l}\text { I usually: Never eat in moderation (1) - always eat in moderation } \\
\text { (5) }\end{array}$ & - & - \\
\hline Risk perception: susceptibility & 5 & $\begin{array}{l}\text { If I do not eat in moderation, my body weight will rise. } \\
\text { Completely disagree (1) - completely agree (5) }\end{array}$ & $3.7(0.6)^{\mathrm{c}}$ & 0.81 \\
\hline Risk perception: severity & 7 & Gaining weight is: Not serious at all (1) - very serious (5) & $3.3(0.6)^{\mathrm{c}}$ & 0.83 \\
\hline Attitude: pros & 10 & $\begin{array}{l}\text { If I eat in moderation, my health will improve. } \\
\text { Completely disagree (1) - completely agree (5) }\end{array}$ & $3.8(0.5)^{\mathrm{c}}$ & 0.94 \\
\hline Attitude: cons & 10 & $\begin{array}{l}\text { If I eat in moderation, I cannot eat everything I want to eat. } \\
\text { Completely disagree (1) - completely agree (5) }\end{array}$ & $2.6(0.6)^{\mathrm{c}}$ & 0.91 \\
\hline Social influence: subjective norm & 4 & $\begin{array}{l}\text { Most people who are important to me, think eating in moderation } \\
\text { is important. Completely disagree (1) - completely agree (5) }\end{array}$ & $3.0(0.7)^{\mathrm{c}}$ & 0.73 \\
\hline
\end{tabular}


Social influence: modelling

Social influence: social support

Self-efficacy

Intention

2

Most people who are important to me, eat in moderation.

Completely disagree (1) - completely agree (5)

moderation. Completely disagree (1) - completely agree (5)

I find it: Very difficult to eat in moderation (1) - very easy to eat in

moderation (5)

1 Do you intend to eat in moderation within the next six months?

Certainly not (1) - certainly (5) $3.3(0.9)^{\mathrm{c}}$

$2.6(0.6)^{\mathrm{c}}$

0.85

${ }^{a}$ This factor was computed in combination with individuals' reported average daily energy intake

${ }^{\mathrm{b}}$ Average daily energy intake in kilocalories

${ }^{\mathrm{c}}$ Mean score on five-point Likert scale

d In the questionnaire, 'product x' was replaced by the food products of the different food categories 


\section{Results \\ Participants}

The study population consisted of 204 men (53.8\%) and 175 women (46.2\%). The mean age was 44.0 years $(\mathrm{SD}=13.5)$ and a majority of the participants $(82.8 \%)$ were living together with a partner, spouse, or child. Further, $29.0 \%$ of the participants had a low level of education, $38.3 \%$ had a medium educational level, and $32.7 \%$ had a high level of education. The mean BMI was $26.7(\mathrm{SD}=5.0)$ and $19.8 \%$ were obese, $39.8 \%$ were overweight, and $40.4 \%$ had a healthy weight. The attrition analysis showed that participants with a low BMI were more likely to drop-out than those with a higher BMI $(\mathrm{OR}=0.93,95 \%$ $\mathrm{CI}=0.88-0.98, P=.012$ ).

\section{Eating in moderation}

The average daily intake of the energy-dense food products was 946.4 kilocalories (kcal) at baseline $(\mathrm{SD}=413.3$, range=170.3-2,272.3) and $906.0 \mathrm{kcal}$ at 6-month follow-up $(\mathrm{SD}=393.3$, range $=110.1-2,175.1)$. The categories of sweet and savory snacks, sandwiches and fillings, and hot and cold beverages were the most important sources for high energy intake. Dairy products, food at dinner, and alcohol contributed the least to the energy intake from energy-dense foods (see Table 1).

\section{Awareness of eating in moderation}

About half of the study population $(51.9 \%)$ were categorized as correctly aware of whether or not they eat in moderation, while $48.1 \%$ were considered to have a wrong perception, i.e. were unaware. Of the aware participants, the majority correctly indicated that they usually ate in moderation $(68.0 \%$ ), while $32.0 \%$ correctly indicated that they usually did not eat in moderation. Of the unaware participants, $75.3 \%$ incorrectly thought that they usually ate in moderation, while $24.7 \%$ incorrectly indicated that they usually did not eat in moderation. Participants who were aware of their intake had a significantly $(\mathrm{t}=-3.68, P=.000)$ lower average daily energy intake (mean=835.1, $\mathrm{SD}=404.1$ ) than the unaware participants (mean=983.4, SD=367.9). Table 2 further shows that there were no significant differences in demographics between the aware and unaware participants.

\section{Correlations between demographics, ICM variables, and eating in moderation}

The Pearson's correlations between the demographic variables, ICM variables, and eating in moderation are shown in Table 3. All demographic variables had a significant negative correlation with baseline eating in moderation. At follow-up, age was the only demographic variable that was not significantly correlated with eating in moderation. Further, being aware of one's own dietary behavior was significantly correlated with a high level of eating in moderation at both baseline as follow-up. Having a high perceived severity and perceived susceptibility correlated significantly with a high level of eating in moderation at baseline and follow-up. Of the motivational factors, attitude pro, subjective norm, and 
social support correlated significantly with eating in moderation at both baseline and follow-up. Intention correlated with nearly all motivational variables and having a high intention was significantly associated with a high level of eating in moderation at baseline and follow-up.

Table 2. Differences in demographics between aware and unaware participants

\begin{tabular}{lccc}
\hline & N Aware (\%) & N Unaware (\%) & Pearson's $\boldsymbol{\chi}^{\mathbf{2}}$ \\
\hline Gender & $103(27.2)$ & $101(26.6)$ & 0.21 \\
Men & $93(24.6)$ & $82(21.6)$ & 2.99 \\
Women & & & \\
Age & $56(14.8)$ & $67(17.7)$ & 5.72 \\
$18-36$ & $67(17.7)$ & $62(16.4)$ & \\
$37-50$ & $73(19.3)$ & $77(20.4)$ & 0.80 \\
$51-66$ & $76(20.1)$ & $70(18.3)$ & \\
BMI & $81(21.4)$ & $36(9.5)$ & \\
Healthy weight & $39(10.3)$ & $51(13.5)$ & \\
Overweight & & $74(19.6)$ & \\
Obese & $58(15.3)$ & $58(15.1)$ & \\
Educational level & $71(18.8)$ & $152(39.9)$ & \\
Low & $67(17.7)$ & $31(8.2)$ & \\
Middle & & & \\
High & $162(42.9)$ & $34(9.0)$ & \\
Household composition & & & \\
Together & &
\end{tabular}


Table 3. Pearson's correlations between the demographic variables, ICM variables, and eating in moderation

\begin{tabular}{|c|c|c|c|c|c|c|c|c|c|c|c|c|c|c|c|c|}
\hline & 1 & 2 & 3 & 4 & 5 & 6 & 7 & 8 & 9 & 10 & 11 & 12 & 13 & 14 & 15 & 16 \\
\hline 1. Gender & 1.00 & & & & & & & & & & & & & & & \\
\hline 2. Educational level & $-.22 * *$ & 1.00 & & & & & & & & & & & & & & \\
\hline 3. Age & $-.24 * *$ & $-.15 * *$ & 1.00 & & & & & & & & & & & & & \\
\hline 4. BMI & $-.10 *$ & $-.16 * *$ & $.32 * *$ & 1.00 & & & & & & & & & & & & \\
\hline $\begin{array}{l}\text { 5. Awareness of own } \\
\text { dietary behavior }\end{array}$ & -.02 & -.01 & -.08 & -.02 & 1.00 & & & & & & & & & & & \\
\hline $\begin{array}{l}\text { 6. Risk perception: } \\
\text { susceptibility }\end{array}$ & $.13 * *$ & -.03 & $.32 * *$ & $.36^{* *}$ & $-.14 * *$ & 1.00 & & & & & & & & & & \\
\hline 7. Risk perception: severity & $.10^{*}$ & .03 & $.09 *$ & $.22 * *$ & $-.11 *$ & $.43 * *$ & 1.00 & & & & & & & & & \\
\hline 8. Attitude: pro & $.22 * *$ & -.02 & .05 & .02 & -.09 & $.45^{* *}$ & $.39 * *$ & 1.00 & & & & & & & & \\
\hline 9. Attitude: con & -.01 & -.06 & $-.15^{* *}$ & $.09 *$ & -.03 & -.01 & $-.12 * *$ & $-.16^{* *}$ & 1.00 & & & & & & & \\
\hline $\begin{array}{l}\text { 10. Social influence: } \\
\text { subjective norm }\end{array}$ & $-.10^{*}$ & $.14 * *$ & $.10^{*}$ & $.11^{*}$ & -.07 & $.27 * *$ & $.38 * *$ & $.29 * *$ & -.06 & 1.00 & & & & & & \\
\hline $\begin{array}{l}\text { 11. Social influence: } \\
\text { modelling }\end{array}$ & -.01 & $.18^{* *}$ & .08 & -.09 & .01 & .09 & $.18 * *$ & $.23 * *$ & $-.29 * *$ & $.48 * *$ & 1.00 & & & & & \\
\hline $\begin{array}{l}\text { 12. Social influence: social } \\
\text { support }\end{array}$ & $-.12 * *$ & $.09^{*}$ & .09 & $.27 * *$ & -.02 & $.24 * *$ & $.29 * *$ & $.17 * *$ & .05 & $.60 * *$ & $.32 * *$ & 1.00 & & & & \\
\hline 13. Self-efficacy & .01 & .05 & .01 & $-.31 * *$ & .02 & $-.21 * *$ & -.05 & -.03 & $-.55^{* *}$ & -.07 & $.30 * *$ & $-.15 * *$ & 1.00 & & & \\
\hline 14. Intention & $.15^{* *}$ & -.05 & $.11 *$ & $.13 * *$ & -.02 & $.40 * *$ & $.28 * *$ & $.38 * *$ & -.08 & $.25 * *$ & $.11 *$ & $.24 * *$ & -.02 & 1.00 & & \\
\hline $\begin{array}{l}15 . \text { Eating in moderation at } \\
\text { baseline }\end{array}$ & $-.21 * *$ & $-.14 * *$ & $-.11^{*}$ & $-.13 * *$ & $.13^{*}$ & $-.20 * *$ & $-.14 * *$ & $-.11^{*}$ & .07 & $-.12 * *$ & $-.14 * *$ & $-.20 * *$ & -.01 & $-.23 * *$ & 1.00 & \\
\hline $\begin{array}{l}\text { 16. Eating in moderation at } \\
\text { six-month follow-up }\end{array}$ & $-.30 * *$ & $-.17 * *$ & -.04 & $-.16^{* *}$ & $.19 * *$ & $-.16^{* *}$ & $-.22 * *$ & $-.13 *$ & .10 & $-.10^{*}$ & -.06 & $-.13 *$ & .03 & $-.21 * *$ & $.72 * *$ & 1.00 \\
\hline
\end{tabular}




\section{Determinants of eating in moderation}

Table 4 shows the results of the multiple linear regression analyses. Note that a lower average daily energy implies a higher level of eating in moderation and vice versa. A negative association in the regression analysis thus implies a positive finding.

Results from the regression analysis with only main effects demonstrate that in step 1 the level of eating in moderation was significantly higher among female participants, participants with a high level of education, older participants, and participants with a high BMI. Results from step 2 show that aware participants and those with a high perceived severity were significantly more likely to eat in moderation. In step 3, these variables remained significant, while social support was found to be the only significant motivational factor. After adding intention in step 4, the significant association of social support disappeared while awareness and perceived severity remained significant predictors. Participants with a high intention to eat in moderation were also significantly more likely to eat in moderation. In the final model, the significant association of intention disappeared, while baseline behavior significantly predicted eating in moderation at follow-up. The explained variance increased from $27 \%$ to $57 \%$ in this last step.

We subsequently performed regression analyses with interaction terms between the dichotomous awareness variable and the ICM variables to examine the moderating role of awareness. Using the pick-a-point approach we examined the associations for aware and unaware participants by switching the $0-1$ coding for the awareness variable. As can be seen in Table 4, among the participants who were aware of their dietary intake, results from step 1 demonstrate that the level of eating in moderation was significantly higher among female participants, participants with a high level of education, older participants, and participants with a high BMI. Results from step 2 show that participants with a high perceived severity were more likely to eat in moderation. The significant association of BMI disappeared in this step. In step 3, the significant association of perceived severity disappeared, while the level of eating in moderation was significantly higher among participants who perceived few cons, a lot of social support, and a negative subjective norm toward eating in moderation. In step 4, all previous associations remained significant while modelling and intention also became significant predictors. After adding baseline behavior in step 5, several significant associations disappeared.

Among the unaware participants, the level of eating in moderation was higher among female participants, participants with a high level of education and participants with a high BMI in step 1. The results from step 2 show no additional or disappearing significant associations. In step 3, perceived susceptibility and age became significant predictors. Of the motivational factors, only self-efficacy had a significant association with eating in moderation. Remarkably, participants with a high self-efficacy were less likely to eat in moderation. The results of step 4 were identical to step 3. In the last step, the significant associations of self-efficacy, perceived susceptibility, and age disappeared, while baseline behavior significantly predicted eating in moderation at follow-up. 
Table 4. Determinants of eating in moderation at 6-month follow-up

\begin{tabular}{|c|c|c|c|c|c|c|c|c|c|c|c|c|c|c|c|}
\hline & \multicolumn{5}{|c|}{ Regression without interaction terms } & \multicolumn{5}{|c|}{ Aware participants } & \multicolumn{5}{|c|}{ Unaware participants } \\
\hline & $\begin{array}{l}\text { Step } 1 \\
\beta\end{array}$ & $\begin{array}{l}\text { Step } 2 \\
\beta\end{array}$ & $\begin{array}{l}\text { Step } 3 \\
\beta\end{array}$ & $\begin{array}{l}\text { Step } 4 \\
\beta\end{array}$ & $\begin{array}{l}\text { Step } 5 \\
\beta\end{array}$ & $\begin{array}{l}\text { Step 1 } \\
\beta\end{array}$ & $\begin{array}{l}\text { Step } 2 \\
\beta\end{array}$ & $\begin{array}{l}\text { Step 3 } \\
\beta\end{array}$ & $\begin{array}{l}\text { Step } 4 \\
\beta\end{array}$ & $\begin{array}{l}\text { Step } 5 \\
\beta\end{array}$ & $\begin{array}{l}\text { Step 1 } \\
\beta\end{array}$ & $\begin{array}{l}\text { Step 2 } \\
\beta\end{array}$ & $\begin{array}{l}\text { Step 3 } \\
\beta\end{array}$ & $\begin{array}{l}\text { Step } 4 \\
\beta\end{array}$ & $\begin{array}{l}\text { Step } 5 \\
\beta\end{array}$ \\
\hline Gender $^{\mathrm{a}}$ & $-.40^{* *}$ & $-.38 * *$ & $-.38 * *$ & $-.36^{* *}$ & $-.15 * *$ & $-.51 * *$ & $-.47 * *$ & $-.41 *$ & $-.37 * *$ & $-.16^{* *}$ & $-.29 * *$ & $-.30 * *$ & $-.28 * *$ & $-.28 * *$ & $-.12 *$ \\
\hline Educational level $^{\mathrm{a}}$ & $-.29 * *$ & $-.28 * *$ & $-.28 * *$ & $-.28 * *$ & $-.12 *$ & $-.33^{* *}$ & $-.31 * *$ & $-.23^{*}$ & $-.25 * *$ & -.11 & $-.26 * *$ & $-.28 * *$ & $-.30 * *$ & $-.29 * *$ & $-.13 *$ \\
\hline Age & $-.12 *$ & $-.12 *$ & $-.11 *$ & -.11 & -.02 & $-.17 *$ & $-.16^{*}$ & -.06 & -.06 & -.04 & -.08 & -.13 & $-.20 * *$ & $-.19 * *$ & -.02 \\
\hline BMI & $-.20 * *$ & $-.18^{*}$ & $-.16^{*}$ & $-.16^{*}$ & $-.10^{*}$ & $-.20 * *$ & -.11 & -.15 & -.13 & -.06 & $-.20 *$ & $-.23 * *$ & $-.14^{*}$ & $-.14 *$ & $-.13^{*}$ \\
\hline $\begin{array}{l}\text { Household } \\
\text { composition }^{\text {a }}\end{array}$ & .03 & .03 & .02 & .02 & .01 & .11 & .11 & .05 & .05 & .06 & -.05 & -.05 & -.06 & -.06 & -.06 \\
\hline Awareness ${ }^{\mathrm{a}}$ & & $.16^{*}$ & $.17 * *$ & $.18^{* *}$ & $.10^{*}$ & & & & & & & & & & \\
\hline $\begin{array}{l}\text { Risk perception: } \\
\text { susceptibility }\end{array}$ & & .04 & .05 & .07 & .06 & & -.06 & -.08 & -.06 & -.01 & & .13 & $.20^{*}$ & $.22 *$ & .12 \\
\hline $\begin{array}{l}\text { Risk perception: } \\
\text { severity }\end{array}$ & & $-.13^{*}$ & $-.12 *$ & $-.12 *$ & $-.10^{*}$ & & $-.19 *$ & -.09 & -.08 & -.08 & & -.08 & -.13 & -.12 & -.10 \\
\hline Attitude: pro & & & .01 & .03 & -.02 & & & -.03 & .02 & -.04 & & & -.03 & -.01 & .00 \\
\hline Attitude: con & & & .10 & .09 & .05 & & & $.23 * *$ & $.22 * *$ & $.12 *$ & & & -.03 & -.03 & .00 \\
\hline $\begin{array}{l}\text { Social influence: } \\
\text { subjective norm }\end{array}$ & & & .11 & .12 & .05 & & & $.20 *$ & $.23 * *$ & $.17 * *$ & & & .01 & .01 & -.11 \\
\hline $\begin{array}{l}\text { Social influence: } \\
\text { modelling }\end{array}$ & & & -.00 & -.02 & .02 & & & -.13 & $-.16^{*}$ & -.07 & & & .16 & .15 & .12 \\
\hline $\begin{array}{l}\text { Social influence: } \\
\text { social support }\end{array}$ & & & $-.12 *$ & -.10 & .02 & & & $-.24 * *$ & $-.22 * *$ & -.09 & & & -.03 & -.02 & .14 \\
\hline Self-efficacy & & & .04 & .05 & .05 & & & -.06 & -.06 & -.01 & & & $.17 *$ & $.18 *$ & .12 \\
\hline Intention & & & & $-.13 *$ & -.05 & & & & $-.19 * *$ & -.08 & & & & -.09 & -.06 \\
\hline $\begin{array}{l}\text { Eating in } \\
\text { moderation at } \\
\text { baseline }\end{array}$ & & & & & $.63^{* *}$ & & & & & $.58 * *$ & & & & & $.58 * *$ \\
\hline $\mathrm{R}^{2}$ & .20 & .24 & .26 & .27 & .57 & .25 & .27 & .36 & .38 & .62 & .25 & .27 & .36 & .38 & .62 \\
\hline
\end{tabular}

$* P<0.05$

${ }^{a}$ Gender: $1=$ male, $2=$ female. Educational level: 1=low, 2=medium, $3=$ high. Household composition: $1=$ alone, $2=$ together. Awareness: $1=$ aware, $2=$ unaware.

38 


\section{Discussion}

The purpose of this study was to examine which factors of the ICM were associated with eating in moderation and whether there was a difference in these factors between Dutch adults who were aware of their dietary behavior and those who were unaware.

In line with other studies on complex health-related behaviors (Bogers et al., 2004; Brug et al., 1994; Glanz, Brug, \& van Assema, 1997; Lechner et al., 2006; Lechner et al., 1997; Lechner et al., 1998; Ronda et al., 2001; van Sluijs et al., 2007), our study shows that half of the participants were unaware of whether or not they eat in moderation and that the majority of these people had an optimistic bias, i.e. they thought to eat in moderation while they did not. In line with the expectations, awareness of dietary behavior was found to be an important factor in the explanation of eating in moderation, since it was a moderator of the association between the ICM variables and behavior (Bogers et al., 2004; Brug et al., 1994; Kloek et al., 2004; Lechner et al., 1997; van Sluijs et al., 2007). The regression analyses with interaction terms showed that only when participants had a correct perception of whether or not they ate in moderation, motivational factors such as attitude, social influence, and intention were significantly associated with eating in moderation.

The finding that motivational factors were not predictive of behavior when people were unaware of their behavior is in line with the findings of previous studies (Bogers et al., 2004; Brug et al., 1994; Kloek et al., 2004; Lechner et al., 1997; van Sluijs et al., 2007). Without an adequate awareness of one's own risk behavior, these unaware people will not perceive a need to change and, accordingly, will have no motivation to change (Bogers et al., 2004; Brug et al., 1994; Lechner et al., 2006; Lechner et al., 1997; Ronda et al., 2001; van Sluijs et al., 2007; Weinstein, 1988). Further, unaware people could, in principle, be highly motivated to eat in moderation and can have a positive attitude. However, these factors are not very likely to predict behavior in this subgroup since they think that they already perform the desired behavior. Lack of awareness can thus act as a barrier in the process of behavior change and limit the effectiveness of interventions aimed to promote eating in moderation (Ronda et al., 2001).

In contrast to these results, the motivational factors were significantly associated with eating in moderation among participants who were aware of their dietary behavior. This implies that people should first be aware of their behavior, before motivational factors become of importance. This is supported by the fact that the explained variance was much higher in the aware group after adding the motivational factors compared to the unaware group. This finding is supported by previous research (Bogers et al., 2004; van Sluijs et al., 2007), which suggests that the predictive value of socio-cognitive theories is higher among people who are aware of their risk behavior. It should, however, be noted that in line with many reviews (Hagger, Chatzisarantis, \& Biddle, 2002; Kidwell \& Jewell, 2008; Sutton, 1994; Trost, Owen, Bauman, Sallis, \& Brown, 2002), baseline behavior was the strongest predictor of eating in moderation. However, it still is important to target the psychosocial cognitions, because by adding past behavior, behavior change is taken into account. 
It is important to recognize that awareness plays an essential role in eating in moderation as this calls for tailored intervention strategies for people who are unaware of their risk behavior (Brug et al., 1994; Lechner et al., 2006; Ronda et al., 2001). Based on our findings, we recommend that future interventions aimed at promoting eating in moderation first aim to achieve an adequate awareness level before targeting motivational factors. Giving feedback on behavior is an effective technique that can be used to create awareness (Bartholomew et al., 2011; Michie et al., 2011; Weinstein, 1988). It has been suggested that this can be done efficiently using web-based computer-tailored interventions, as these can raise people's awareness relatively easily by providing individualized feedback about their behavior (Oenema \& Brug, 2003).

Our findings were not completely in line with our hypothesis, since two significant associations were not in the expected direction among the unaware participants. The finding that having a high risk perception was associated with a lower level of eating in moderation can, however, be explained by the fact that these individuals were unaware of their dietary behavior and most of them incorrectly thought that they ate in moderation. As suggested by a review about factors affecting food choice (Pollard, Kirk, \& Cade, 2002), these unaware participants probably had an optimistic bias and consequently may have thought that they were less at risk (Weinstein \& Lyon, 1999). The finding that a high self-efficacy for eating in moderation was associated with a lower level of eating in moderation can also be related to this optimistic bias (Pollard et al., 2002; Weinstein \& Lyon, 1999). The largest part of the unaware participants thought that they already performed the desired behavior and they may have experienced that this behavior was relatively easy to achieve, which may be reflected in a high self-efficacy. However, in this case self-efficacy is not related to actual eating in moderation.

The finding that there was no significant relationship between self-efficacy and eating in moderation among the aware participants may be explained by the manner in which self-efficacy was measured. Thinking that something is easy to do, does not necessarily mean that one is able to perform the behavior successfully. Another remarkable finding among the aware participants was that the level of eating in moderation was lower among participants who indicated that important others thought that they should eat in moderation (i.e. subjective norm). This finding can, however, be explained by the presence of a suppressor effect (Lancaster, 1999): when the univariate association between two variables disappears or even turns around when adding other variables. Our results show that this happened for subjective norm, since a Pearson correlation analysis showed that the level of eating in moderation was significantly higher among participants who perceived a positive norm toward eating in moderation $(r=-.10, P=.006)$, while this relation was reversed in the regression analysis.

The results of this study should be interpreted in the light of three important limitations. The use of the median split to classify the daily energy intake into eating in moderation versus not eating in moderation was somewhat arbitrary. We have, however, 
three good reasons for using this procedure. First, about $50 \%$ of Dutch adults have an energy intake higher than the daily recommended energy intake (van Rossum, Fransen, Verkaik-Kloosterman, Buurma-Rethans, \& Ocké, 2011); therefore, we expected to have the same distribution in our sample. Second, the median split was made separately for men and women, as men are allowed to have a higher daily energy intake than women. Third, we also verified this approach using a more conservative approach in which we classified the average daily energy intake of the lowest $67 \%$ into eating in moderation and the top $33 \%$ into not eating in moderation. Performing the analyses using these cut-off points resulted in findings in the same direction. Another aspect to mention is the dichotomous classification of awareness, through which both awareness subgroups consisted of people who did and who did not eat in moderation. The subgroup of aware participants, for example, included both participants who correctly indicated that they usually ate in moderation as well as participants who were correctly aware of the fact that they did not eat in moderation. We did, however, also perform separate regression analyses for the four awareness subgroups. The results of these analyses did not demonstrate obvious differences between these groups. This implies that being aware is important in itself, regardless of whether this is the (un)awareness of eating in moderation or not eating in moderation. Furthermore, the use of the average daily energy intake as an indicator for eating in moderation can be considered as suboptimal. However, eating in moderation is a complex behavior which could be measured in many different ways. Currently, it is unknown what the best indicator for this behavior is and, eventually, everything comes down to the energy intake. We further also measured all aspects of eating in moderation in our questionnaire, such as the frequency, quantity, portion size and type of product. Finally, it should be noted that this is the first study that has identified the determinants of eating in moderation and examined the moderating role of awareness for this behavior. Further research on this behavior is recommended in order to replicate our findings.

\section{Conclusions}

The aim of this study was to identify determinants of eating in moderation and to study differential effects based on people's level of awareness in order to be able to target the most important determinants in interventions aimed at preventing overweight and obesity. This study shows that awareness of one's own risk behavior is a key factor in eating in moderation. Misperception of one's own risk behavior may act as a barrier when promoting eating in moderation as unaware people will not perceive a need to change and have no motivation to change. This can limit the efficacy of interventions that only focus on motivational factors. Hence, interventions aimed at promoting eating in moderation should first focus on achieving an adequate awareness level of one's own dietary behavior before targeting motivational factors, such as attitude, social influence, and intention. By following this sequence, the adoption of a moderate eating pattern can be promoted effectively in, for example, web-based computer-tailored interventions. 


\section{CHAPTER 3}

Are clusters of dietary patterns and cluster membership
stable over time? Results of a longitudinal cluster analysis
study

Published as: Walthouwer, M., Oenema, A., Soetens, K., Lechner, L., \& de Vries, H. (2014). Are clusters of dietary patterns and cluster membership stable over time? Results of a longitudinal cluster analysis study. Appetite, 82, 154-159. 


\begin{abstract}
Introduction: Developing nutrition education interventions based on clusters of dietary patterns can only be done adequately when it is clear if distinctive clusters of dietary patterns can be derived and reproduced over time, if cluster membership is stable, and if it is predictable which type of people belong to a certain cluster. Hence, this study aimed to: (1) identify clusters of dietary patterns among Dutch adults, (2) test the reproducibility of these clusters and stability of cluster membership over time, and (3) identify sociodemographic predictors of cluster membership and cluster transition.

Methods: This study had a longitudinal design with online measurements at baseline $(\mathrm{N}=483)$ and six months follow-up $(\mathrm{N}=379)$. Dietary intake was assessed with a validated food frequency questionnaire. A hierarchical cluster analysis was performed, followed by a $\mathrm{K}$-means cluster analysis. Multinomial logistic regression analyses were conducted to identify the sociodemographic predictors of cluster membership and cluster transition.

Results: At baseline and follow-up, a comparable three-cluster solution was derived, distinguishing a healthy, moderately healthy, and unhealthy dietary pattern. Male and lower educated participants were significantly more likely to have a less healthy dietary pattern. Further, 251 (66.2\%) participants remained in the same cluster, 45 (11.9\%) participants changed to an unhealthier cluster, and $83(21.9 \%)$ participants shifted to a healthier cluster. Men and people living alone were significantly more likely to shift towards a less healthy dietary pattern.

Conclusions: Distinctive clusters of dietary patterns can be derived. Yet, cluster membership is unstable and only few sociodemographic factors were associated with cluster membership and cluster transition. These findings imply that clusters based on dietary intake may not be suitable as a basis for nutrition education interventions.
\end{abstract}

Keywords: cluster analysis, dietary patterns, adults, longitudinal study, determinants 


\section{Introduction}

A healthy diet protects against several types of cancer, cardiovascular diseases, and overweight (WHO, 2002, 2003). Hence, it is important to develop nutrition education interventions that can promote healthy diets consisting of, for example, a limited intake of fats, sugars, and salt and a high intake of whole grains, lean meat, fruits, and vegetables (WHO, 2002, 2003, 2008). More and more it has been suggested that the dietary advices of such interventions can be enhanced when these are targeting whole dietary patterns instead of separate food products, since people do not consume foods in isolation but as part of an overall dietary pattern (Correa Leite et al., 2003; Hearty \& Gibney, 2009, 2013; Millen et al., 2001; Millen et al., 2005; Newby et al., 2003; Newby \& Tucker, 2004; Togo, Osler, Sørensen, \& Heitmann, 2001). Interventions that focus on a single nutrient or food product are unable to account for how these products act in combination and accordingly are less likely to provide tangible dietary advice (Millen et al., 2001; Newby \& Tucker, 2004; Quatromoni et al., 2002; Togo et al., 2001; Wang, Worsley, \& Hodgson, 2012).

Cluster analysis is a commonly used method to categorize people into nonoverlapping dietary pattern groups according to the similarity of foods consumed (Hearty \& Gibney, 2009; Newby \& Tucker, 2004). It can be used within nutrition education interventions to, for example, provide people with a more tailored dietary advice (Hearty \& Gibney, 2009, 2013; Newby \& Tucker, 2004; Togo et al., 2001). In order to be able to do this adequately, three important conditions should be met. First, it should be possible to derive distinctive clusters of dietary patterns, because otherwise no appropriate basis will be available for these interventions. Related to this, it is also important that these derived clusters are reproducible over time (Devlin, McNulty, Nugent, \& Gibney, 2012). Second, it is also necessary to know whether or not cluster membership is stable (Northstone, Smith, Newby, \& Emmett, 2013). For example, much changeability in individuals' dietary patterns over time could imply that it may be less effective to base interventions on clusters of dietary patterns. Third, the efficiency of nutrition education interventions can further also be improved when it is predictable which type of people belong to a certain cluster of dietary pattern, as this makes it possible to conduct these interventions in high-risk groups who have unhealthy dietary patterns (Correa Leite et al., 2003; Grieger, Scott, \& Cobiac, 2012; Gubbels, van Assema, \& Kremers, 2013; Quatromoni et al., 2002). These three aspects are essential to know and together largely determine the usefulness of cluster analysis within nutrition education interventions. If one of these three conditions is not met, developing interventions based on clusters of dietary patterns may not be of added value.

To date, much is unknown about the reproducibility, stability, and predictability of clusters of dietary patterns because most studies have used cross-sectional data (Devlin et al., 2012; Grieger et al., 2012; Newby et al., 2003; Northstone et al., 2013; Togo et al., 2001). These studies suggest that it is possible to derive clusters of dietary patterns ranging from, for example, very healthy to unhealthy (Devlin et al., 2012; Kant, 2004; Newby \& Tucker, 2004), but there is a lack of insight into the reproducibility of these clusters over 
time (Devlin et al., 2012). Predictors of cluster membership (i.e. dietary patterns) have also mostly been examined using cross-sectional data. A review based on cross-sectional studies, for example, showed that women and people with a high education level are more likely to have a healthy dietary pattern (Kant, 2004). However, an important finding of a longitudinal study is that cluster membership is not always stable and dietary patterns can thus change over time (Northstone et al., 2013). In addition, little is known about the predictors of cluster transition.

Because the results above are largely based on cross-sectional studies and much is unknown about clusters of dietary patterns over time, it can be concluded that more longitudinal cluster analysis research is needed. Hence, the aim of this study was to (1) identify clusters of dietary patterns among Dutch adults, (2) test the reproducibility of these clusters and the stability of cluster membership over time, and (3) identify sociodemographic predictors of cluster membership and cluster transition.

\section{Material and methods}

\section{Study design and participants}

This study was conducted as a longitudinal observational study with measurements at baseline (September 2011) and six months follow-up (February 2012). The study was fully conducted through the Internet and participants were recruited from among the members of an internet research panel (Flycatcher, the Netherlands). Participants were eligible to participate if they (a) were between 18 and 65 years old, (b) had a job, (c) had access to the Internet, and (d) were able to read and understand Dutch. At baseline, 942 members were approached, of which 483 participated (response=51\%). At follow-up, 379 of these 483 completed the online questionnaire (response=78\%). For the present study, only participants who completed the questionnaires at both measurement points were included in the analyses.

\section{Questionnaire}

At baseline and follow-up, participants' dietary intake was assessed with a validated food frequency questionnaire consisting of 66 items (van Assema et al., 2001). This questionnaire measured the intake levels of mainly energy-dense products from eight different food categories (i.e. dairy products, sandwiches and fillings, food at dinner, sweet and savory snacks, hot and cold beverages, alcohol, fruit, and vegetables). Each food product within a category was assessed with two or three items in order to capture the frequency (i.e. number of days per week), quantity (i.e. amount per day), and if applicable type of product (e.g. use of skimmed, semi-skimmed, or whole milk). By combining these three types of items with the energy value of each food product, the average daily intake of calories from energy-dense food products was calculated. For each food product, we also calculated the average daily frequency of consumption. These frequency scores were subsequently standardized to z-scores to ensure that all food products had equal weights in 
the cluster analysis and the clusters were not influenced by foods with high specific gravity (e.g. beverages) (Crozier, Robinson, Borland, \& Inskip, 2006; Pryer et al., 2001; Wishart, 2001). A complete overview of all included foods can be found in Table 1.

At baseline, several sociodemographic characteristics were assessed as well, including gender, age, educational level, household composition, and height and body weight to calculate BMI. Educational level (i.e. the highest level of education completed) was categorized into three categories: low (less than primary education, primary education, lower secondary education), medium (upper secondary education, post-secondary nontertiary education, short-cycle tertiary education), and high (bachelor's level, master's level, doctoral level) (UNESCO Institute for Statistics, 2012). Household composition was dichotomized into living alone versus living together.

\section{Statistical analyses}

A logistic regression analysis was performed to examine the possible presence of selective attrition between baseline and follow-up regarding the sociodemographic variables. To describe the demographic characteristics of the study population, descriptive statistics and frequencies were used. These were also used to describe the different cluster solutions of the cluster analysis.

To reduce the subjectivity inherent to cluster analysis, we first performed a hierarchical cluster analysis using Ward's method as clustering method (Milligan \& Cooper, 1987) followed by a K-means cluster analysis. To determine the number of cluster solutions in the hierarchical cluster analysis, the squared Euclidean distances of the agglomeration schedule were displayed using a scree plot, where higher ratios indicate a better separation of clusters. The dendogram of the hierarchical cluster analysis also aided in this decision. Next, to determine the actual number of cluster solutions in the K-means cluster analysis, we conducted the analysis with a variation of two to five cluster solutions using different starting positions (i.e. different sorting of variables and order of variables in dataset). One-way ANOVA's with Tukey post-hoc tests were performed to assess differences between the clusters in the mean consumption of each food product in order to validate the cluster solutions and to determine whether or not the identified clusters were significantly different from each other (Stricker et al., 2013). Labels to the clusters were given based on the most frequently consumed food products within each cluster (Newby \& Tucker, 2004). This whole procedure was done by two researchers independently from each other and discussed with a third independent researcher. We also examined changes in dietary patterns over time by creating a variable that reflected whether or not participants changed from cluster between baseline and follow-up ( $1=$ moved to a healthier cluster, $2=$ moved to a less healthy cluster; $3=$ =remained in same cluster). To examine to which degree participants changed from cluster between baseline and follow-up, we performed a cross-tab analysis using Cohen's Kappa and computed Spearman's correlation. Finally, multinomial logistic regression analyses were conducted to identify which factors (i.e. 
demographics and BMI) were associated with being a member of a cluster at baseline and follow-up and for cluster transition.

This whole procedure was applied to the data of both the baseline and follow-up measurement. All statistical analyses were conducted using SPSS 19.0, applying a significance level of 0.05 .

\section{Results}

\section{Participants}

The mean age of the 379 respondents was 44.0 years $(\mathrm{SD}=13.5)$. Men $(53.8 \%)$ were slightly overrepresented in this sample. The mean BMI was 26.7 ( $\mathrm{SD}=5.0)$. Further, 29.0\% had a low level of education, 38.3\% had a medium level of education, and $32.7 \%$ was highly educated. The majority of the participants (82.8\%) lived together with a partner, spouse, or child. The attrition analysis showed that attrition was significantly higher among participants with a lower $\mathrm{BMI}(\mathrm{OR}=0.93,95 \% \mathrm{CI}=0.88-0.98, P=.012)$.

\section{Cluster profiles}

Following the two-step cluster analysis and after testing several options, a three-cluster solution was derived at baseline, which we labeled 'healthy' (cluster 1), 'moderately healthy' (cluster 2), and 'unhealthy' (cluster 3) (see Table 1). Cluster 1 ( $\mathrm{N}=149 ; 39.3 \%)$ was named 'healthy' because members of this cluster consumed a high amount of fruit and vegetables, low-fat yoghurt, and brown bread. They further had a relatively low daily consumption of snacks, alcohol, and sweetened drinks. The average daily intake of calories from energy-dense food products in this cluster was 757.0 ( $\mathrm{SD}=293.7$ ) kilocalories (kcal). Cluster $2(\mathrm{~N}=171 ; 45.1 \%)$ was named 'moderately healthy' because members of this cluster had a high daily consumption of white bread, high-fat butter, sugar sweetened drinks, and only a little fruit and vegetables. However, this cluster did not reveal a very pronounced healthy or unhealthy pattern. The average daily intake of calories from energy-dense food products in this cluster was $954.7(\mathrm{SD}=385.8)$ kcal. Cluster $3(\mathrm{~N}=59 ; 15.6 \%)$ was considered to be the 'unhealthy' cluster, with high levels of snack consumption, tea or coffee with additions of milk and/or sugar, and chocolate spread. Participants in this cluster also had a higher than average daily consumption of brown bread and reduced-fat pudding, which is incongruent with this pattern. The average daily intake of calories from energydense food products in this cluster was 1,453.7 ( $\mathrm{SD}=430.8) \mathrm{kcal}$.

At follow-up, a comparable three-cluster solution was derived with a healthy $(\mathrm{N}=185 ; 48.8 \%)$, a moderately healthy $(\mathrm{N}=151 ; 39.8 \%)$, and an unhealthy cluster $(\mathrm{N}=43$; $11.4 \%$ ) as well (see Table 2). In line with the baseline clusters, participants in the healthy cluster had the lowest average daily intake of calories from energy-dense food products (Mean=832.0, SD=339.8), followed by the moderately healthy (Mean=878.1, SD=373.5) and unhealthy cluster (Mean=1,375.7, $\mathrm{SD}=401.6)$. There were a few small differences in the composition within the clusters among dairy products and sandwiches and fillings. 
Table 1. Cluster solution at baseline: mean z-scores for each food product per cluster

\begin{tabular}{|c|c|c|c|}
\hline & $\begin{array}{c}\text { Cluster 1: Healthy } \\
(\mathrm{N}=149)\end{array}$ & $\begin{array}{l}\text { Cluster 2: Moderately } \\
\text { healthy }(\mathrm{N}=171)\end{array}$ & $\begin{array}{c}\text { Cluster 3: Unhealthy } \\
\qquad(\mathrm{N}=59)\end{array}$ \\
\hline \multicolumn{4}{|l|}{ Dairy products } \\
\hline Full-fat milk/chocolate milk & -0.11 & -0.06 & 0.11 \\
\hline Reduced-fat milk/chocolate milk & $0.07^{\mathrm{a}}$ & $-0.20^{\mathrm{a}, \mathrm{b}}$ & $0.26^{\mathrm{b}}$ \\
\hline Full-fat yoghurt & -0.03 & -0.05 & -0.11 \\
\hline Reduced-fat yoghurt & $0.44^{\mathrm{a}, \mathrm{b}}$ & $-0.36^{\mathrm{a}}$ & $-0.12^{\mathrm{b}}$ \\
\hline Sugar-sweetened dairy beverages & $-0.16^{\mathrm{a}}$ & -0.04 & $0.33^{\mathrm{a}}$ \\
\hline Full-fat pudding & $-0.18^{\mathrm{a}}$ & 0.01 & $0.09^{\mathrm{a}}$ \\
\hline Reduced-fat pudding & $-0.05^{\mathrm{a}}$ & $-0.16^{\mathrm{b}}$ & $0.41^{\mathrm{a}, \mathrm{b}}$ \\
\hline \multicolumn{4}{|l|}{ Sandwiches and fillings } \\
\hline White bread & $-0.24^{\mathrm{a}, \mathrm{b}}$ & $0.11^{\mathrm{a}}$ & $0.02^{\mathrm{b}}$ \\
\hline Brown/whole meal bread & $0.26^{\mathrm{a}}$ & $-0.42^{\mathrm{a}, \mathrm{b}}$ & $0.55^{\mathrm{b}}$ \\
\hline High-fat meat products & -0.17 & 0.06 & 0.09 \\
\hline Lean meat products & 0.03 & $-0.12^{\mathrm{a}}$ & $0.25^{\mathrm{a}}$ \\
\hline Chocolate spread & $-0.09^{\mathrm{a}}$ & $-0.23^{\mathrm{b}}$ & $0.77^{\mathrm{a}, \mathrm{b}}$ \\
\hline High-fat butter spread & $-0.24^{\mathrm{a}}$ & $0.28^{b}$ & $-0.24^{\mathrm{a}, \mathrm{b}}$ \\
\hline Low-fat butter spread & $0.12^{\mathrm{a}}$ & $-0.38^{\mathrm{a}}$ & $0.82^{\mathrm{a}}$ \\
\hline \multicolumn{4}{|l|}{ Food at dinner } \\
\hline Processed meat & $-0.34^{\mathrm{a}}$ & $-0.03^{\mathrm{a}}$ & $0.92^{\mathrm{a}}$ \\
\hline Cheese & $-0.00^{\mathrm{a}}$ & $-0.19^{b}$ & $0.48^{a, b}$ \\
\hline Gravy & $-0.33^{\mathrm{a}}$ & $-0.01^{\mathrm{a}}$ & $0.82^{\mathrm{a}}$ \\
\hline \multicolumn{4}{|l|}{ Sweet and savory snacks } \\
\hline Fried snacks & $-0.27^{\mathrm{a}}$ & $-0.04^{\mathrm{a}}$ & $0.56^{\mathrm{a}}$ \\
\hline Peanuts and potato chips & $-0.29^{\mathrm{a}}$ & $-0.05^{b}$ & $0.75^{\mathrm{a}, \mathrm{b}}$ \\
\hline Cheese/sausages snacks & $-0.19^{\mathrm{a}, \mathrm{b}}$ & $0.06^{\mathrm{a}}$ & $0.15^{b}$ \\
\hline Cake & -0.05 & $-0.14^{\mathrm{a}}$ & $0.30^{\mathrm{a}}$ \\
\hline Chocolates, candy bars & 0.03 & $-0.15^{\mathrm{a}}$ & $0.21^{\mathrm{a}}$ \\
\hline Biscuits, cookies & $0.13^{\mathrm{a}}$ & $-0.25^{b}$ & $0.31^{\mathrm{a}, \mathrm{b}}$ \\
\hline \multicolumn{4}{|l|}{ Fruit and vegetables } \\
\hline Fruit & $0.69^{\mathrm{a}}$ & $-0.54^{\mathrm{a}}$ & $-0.19^{\mathrm{a}}$ \\
\hline Vegetables (cooked) & $0.42^{\mathrm{a}, \mathrm{b}}$ & $-0.35^{\mathrm{a}}$ & $-0.04^{\mathrm{a}}$ \\
\hline Vegetables (raw) & $0.38^{a, b}$ & $-0.26^{\mathrm{a}}$ & $-0.25^{\mathrm{b}}$ \\
\hline \multicolumn{4}{|l|}{ Hot and cold beverages } \\
\hline Coffee with milk and/or sugar & $-0.35^{\mathrm{a}}$ & $0.09^{\mathrm{a}}$ & $0.60^{\mathrm{a}}$ \\
\hline Tea with sugar or honey & $-0.20^{\mathrm{a}}$ & -0.07 & $0.49^{\mathrm{a}}$ \\
\hline Sugar sweetened drinks & $-0.29^{\mathrm{a}}$ & $0.20^{\mathrm{a}}$ & 0.03 \\
\hline Alcohol & $-0.22^{\mathrm{a}, \mathrm{b}}$ & $0.06^{\mathrm{a}}$ & $0.08^{\mathrm{b}}$ \\
\hline
\end{tabular}

One-way ANOVA's with Tukey post-hoc tests showed that the clusters significantly differed on nearly all included food products at both measurement points (see Table 1 and 2), indicating that the clusters were different from each other. For example, participants in the healthy baseline cluster had a significantly higher daily consumption of raw vegetables compared to participants in the two other baseline clusters. Further, the 
difference in the average daily intake of calories from energy-dense food products was also significantly different between the clusters at both baseline $(\mathrm{F}=76.324, P=.000)$ as followup $(\mathrm{F}=36.678, P=.000)$.

Table 2. Cluster solution at follow-up: mean z-scores for each food product per cluster

\begin{tabular}{|c|c|c|c|}
\hline & $\begin{array}{l}\text { Cluster 1: Healthy } \\
\qquad(\mathrm{N}=\mathbf{1 8 5})\end{array}$ & $\begin{array}{c}\text { Cluster 2: Moderately } \\
\text { healthy }(\mathrm{N}=151)\end{array}$ & $\begin{array}{c}\text { Cluster 3: Unhealthy } \\
\qquad(\mathrm{N}=43)\end{array}$ \\
\hline \multicolumn{4}{|l|}{ Dairy products } \\
\hline Full-fat milk/chocolate milk & $-0.12^{\mathrm{a}}$ & -0.03 & $0.12^{\mathrm{a}}$ \\
\hline Reduced-fat milk/chocolate milk & $-0.08^{\mathrm{a}}$ & $-0.15^{\mathrm{b}}$ & $0.85^{\mathrm{a}, \mathrm{b}}$ \\
\hline Full-fat yoghurt & 0.01 & -0.11 & -0.10 \\
\hline Reduced-fat yoghurt & $0.08^{\mathrm{a}}$ & $-0.17^{\mathrm{a}}$ & 0.16 \\
\hline Sugar-sweetened dairy beverages & $-0.11^{\mathrm{a}}$ & $-0.08^{\mathrm{b}}$ & $0.39^{\mathrm{a}, \mathrm{b}}$ \\
\hline Full-fat pudding & -0.07 & -0.05 & 0.06 \\
\hline Reduced-fat pudding & $-0.21^{\mathrm{a}}$ & $-0.12^{\mathrm{b}}$ & $1.12^{\mathrm{a}, \mathrm{b}}$ \\
\hline \multicolumn{4}{|l|}{ Sandwiches and fillings } \\
\hline White bread & $-0.25^{\mathrm{a}}$ & $0.24^{\mathrm{a}, \mathrm{b}}$ & $-0.20^{\mathrm{b}}$ \\
\hline Brown/whole meal bread & $0.46^{\mathrm{a}}$ & $-0.77^{\mathrm{a}, \mathrm{b}}$ & $0.71^{\mathrm{b}}$ \\
\hline High-fat meat products & $-0.20^{\mathrm{a}}$ & $0.13^{\mathrm{a}}$ & 0.03 \\
\hline Lean meat products & $0.17^{\mathrm{a}}$ & $-0.27^{\mathrm{a}}$ & 0.12 \\
\hline Chocolate spread & $-0.20^{\mathrm{a}}$ & $-0.12^{\mathrm{b}}$ & $1.17^{\mathrm{a}, \mathrm{b}}$ \\
\hline High-fat butter spread & -0.05 & 0.02 & 0.09 \\
\hline Low-fat butter spread & $0.31^{\mathrm{a}}$ & $-0.49^{\mathrm{a}, \mathrm{b}}$ & $0.41^{\mathrm{b}}$ \\
\hline \multicolumn{4}{|l|}{ Food at dinner } \\
\hline Processed meat & $-0.28^{\mathrm{a}}$ & $0.04^{\mathrm{a}}$ & $0.98^{\mathrm{a}}$ \\
\hline Cheese & $0.26^{\mathrm{a}}$ & $-0.39^{\mathrm{a}, \mathrm{b}}$ & $0.16^{b}$ \\
\hline Gravy & $-0.13^{\mathrm{a}}$ & 0.02 & $0.39^{\mathrm{a}}$ \\
\hline \multicolumn{4}{|l|}{ Sweet and savory snacks } \\
\hline Fried snacks & $-0.26^{\mathrm{a}}$ & $0.06^{\mathrm{a}}$ & $0.68^{\mathrm{a}}$ \\
\hline Peanuts and potato chips & $-0.07^{\mathrm{a}}$ & $-0.05^{\mathrm{b}}$ & $0.35^{\mathrm{a}, \mathrm{b}}$ \\
\hline Cheese/sausages snacks & $-0.13^{\mathrm{a}}$ & $-0.01^{b}$ & $0.34^{\mathrm{a}, \mathrm{b}}$ \\
\hline Cake & $-0.09^{\mathrm{a}}$ & $-0.14^{b}$ & $0.54^{\mathrm{a}, \mathrm{b}}$ \\
\hline Chocolates, candy bars & $-0.09^{\mathrm{a}}$ & $-0.13^{\mathrm{b}}$ & $0.54^{\mathrm{a}, \mathrm{b}}$ \\
\hline Biscuits, cookies & $-0.07^{\mathrm{a}}$ & $-0.07^{\mathrm{b}}$ & $0.44^{\mathrm{a}, \mathrm{b}}$ \\
\hline \multicolumn{4}{|l|}{ Fruit and vegetables } \\
\hline Fruit & $0.28^{\mathrm{a}}$ & $-0.35^{\mathrm{a}, \mathrm{b}}$ & $0.01^{\mathrm{b}}$ \\
\hline Vegetables (cooked) & $0.43^{\mathrm{a}, \mathrm{b}}$ & $-0.47^{\mathrm{a}}$ & $-0.17^{\mathrm{b}}$ \\
\hline Vegetables (raw) & $0.30^{a, b}$ & $-0.28^{a}$ & $-0.38^{\mathrm{b}}$ \\
\hline \multicolumn{4}{|l|}{ Hot and cold beverages } \\
\hline Coffee with milk and/or sugar & $-0.08^{\mathrm{a}}$ & $-0.12^{b}$ & $0.76^{\mathrm{a}, \mathrm{b}}$ \\
\hline Tea with sugar or honey & $-0.08^{\mathrm{a}}$ & -0.04 & $0.25^{\mathrm{a}}$ \\
\hline Sugar sweetened drinks & $-0.29^{\mathrm{a}, \mathrm{b}}$ & $0.26^{\mathrm{a}}$ & $0.16^{b}$ \\
\hline Alcohol & -0.08 & 0.06 & -0.11 \\
\hline
\end{tabular}




\section{Predictors of cluster membership}

The multinomial logistic regression analysis showed that only gender and level of education were significantly associated with cluster membership at baseline (see Table 3). Women were more likely than men to be in the healthy cluster compared to the moderately healthy $(\mathrm{OR}=2.11,95 \% \mathrm{CI}=1.18-3.77, P=.004)$ or unhealthy cluster $(\mathrm{OR}=4.94,95 \%$ $\mathrm{CI}=1.91-12.81, P=.000$ ). Participants with a low educational level were more likely than highly educated participants to be in the unhealthy cluster compared to the healthy $(\mathrm{OR}=9.38,95 \% \mathrm{CI}=2.99-29.42, P=.000)$ or moderately healthy cluster ( $\mathrm{OR}=5.51,95 \%$ $\mathrm{CI}=2.64-11.50, P=.001)$.

At follow-up, only level of education was significantly associated with cluster membership (see Table 4). Participants with a low educational level were more likely than highly educated participants to be in the unhealthy cluster compared to the healthy $(\mathrm{OR}=4.96,95 \% \mathrm{CI}=1.41-17.41, P=.010)$ or moderately healthy cluster $(\mathrm{OR}=3.53,95 \%$ $\mathrm{CI}=1.85-6.71, P=.000)$.

Table 3. Predictors of cluster membership at baseline

\begin{tabular}{lccc}
\hline Demographics & $\begin{array}{c}\text { Cluster 1: Healthy } \\
(\mathbf{N = 1 4 9})\end{array}$ & $\begin{array}{c}\text { Cluster 2: Moderately } \\
\text { healthy }(\mathbf{N = 1 7 1})\end{array}$ & $\begin{array}{c}\text { Cluster 3: Unhealthy } \\
(\mathbf{N}=59)\end{array}$ \\
\hline Gender $(\%)$ & $63(42.3)^{\mathrm{a}, \mathrm{b}}$ & $96(56.1)^{\mathrm{a}}$ & $45(76.3)^{\mathrm{b}}$ \\
$\quad$ Male & $86(57.7)$ & $75(43.9)$ & $14(23.7)$ \\
$\quad$ Female & & & \\
Educational level (\%) & $21(14.1)^{\mathrm{a}, \mathrm{b}}$ & $62(36.3)^{\mathrm{a}}$ & $27(45.8)^{\mathrm{b}}$ \\
$\quad$ Low & $61(40.9)^{\mathrm{a}, \mathrm{b}}$ & $63(36.8)^{\mathrm{a}}$ & $21(35.6)^{\mathrm{b}}$ \\
$\quad$ Middle & $67(45.0)$ & $46(26.9)$ & $11(18.6)$ \\
$\quad$ High & & & \\
Household composition & $31(20.8)$ & $26(15.2)$ & $8(13.6)$ \\
$(\%)$ & $118(79.2)$ & $145(84.8)$ & $51(86.4)$ \\
$\quad$ Alone & & & \\
$\quad$ Together & $42.4(14.1)$ & $44.3(13.0)$ & $43.5(12.8)$ \\
Age mean (SD) & $26.6(5.6)$ & $26.8(4.7)$ & $26.6(4.6)$ \\
BMI mean (SD) & &
\end{tabular}

$\overline{\mathrm{a}, \mathrm{b}}$ Values within a row with identical letters indicate a significant predictor of cluster membership $(P<0.05)$, as derived from multinomial logistic regression analyses.

\section{Cluster membership over time}

The level of agreement between the baseline cluster solution and the follow-up cluster solution was fairly low $(r=0.456, P=.000$; Cohen's Kappa $=0.45, P=.000)$. This indicates that cluster membership over time was not stable and that some participants changed from cluster between baseline and follow-up. Table 5 provides an overview of all cluster changes between baseline and follow-up. In total, 251 (66.2\%) participants remained in the same cluster, $45(11.9 \%)$ participants changed to an unhealthier cluster, and $83(21.9 \%)$ participants shifted to a healthier cluster. In the healthy cluster at baseline $(\mathrm{N}=149)$, most participants were in the same cluster after 6 months, whereas about one quarter changed to a less healthy cluster. In the moderately healthy cluster $(\mathrm{N}=171)$, the majority remained in 
the cluster, $52(30.4 \%)$ changed to the healthy cluster, and $10(5.8 \%)$ changed to the unhealthy cluster. Of the 59 participants in the unhealthy cluster, about half remained in the cluster after 6 months, while $52.5 \%$ changed to a healthier cluster.

Table 4. Predictors of cluster membership at follow-up

\begin{tabular}{|c|c|c|c|}
\hline Demographics & $\begin{array}{l}\text { Cluster 1: Healthy } \\
\qquad(\mathrm{N}=185)\end{array}$ & $\begin{array}{c}\text { Cluster 2: Moderately } \\
\text { healthy }(\mathrm{N}=151)\end{array}$ & $\begin{array}{c}\text { Cluster 3: Unhealthy } \\
\qquad(\mathrm{N}=43)\end{array}$ \\
\hline \multicolumn{4}{|l|}{ Gender (\%) } \\
\hline Male & $102(55.1)$ & $70(46.4)$ & $32(74.4)$ \\
\hline Female & $83(44.9)$ & $81(53.6)$ & $11(25.6)$ \\
\hline \multicolumn{4}{|c|}{ Educational level (\%) } \\
\hline Low & $40(21.6)^{\mathrm{a}, \mathrm{b}}$ & $53(35.1)^{\mathrm{a}}$ & $17(39.5)^{\mathrm{b}}$ \\
\hline Middle & $61(33.0)$ & $64(42.4)$ & $20(46.5)$ \\
\hline High & $84(45.4)$ & $34(22.5)$ & $6(14.0)$ \\
\hline \multicolumn{4}{|c|}{ Household composition } \\
\hline$(\%)$ & $37(20.0)$ & $27(17.9)$ & $1(2.3)$ \\
\hline Alone & $148(80.0)$ & $124(82.1)$ & $42(97.7)$ \\
\hline \multicolumn{4}{|l|}{ Together } \\
\hline Age mean (SD) & $44.1(13.5)$ & $42.5(13.7)$ & $43.9(12.0)$ \\
\hline BMI mean (SD) & $26.8(5.2)$ & $26.8(5.0)$ & $26.0(4.3)$ \\
\hline
\end{tabular}

${ }^{\mathrm{a}, \mathrm{b}}$ Values within a row with identical letters indicate a significant predictor of cluster membership $(P<0.05)$, as derived from multinomial logistic regression analyses

Table 5. Cluster membership over time: baseline by follow-up cluster membership

\begin{tabular}{llll}
\hline Baseline & Follow-up & & \\
\cline { 2 - 4 } & Healthy & Moderately healthy & Unhealthy \\
\hline Healthy & $114(76.5 \%)$ & $30(20.1 \%)$ & $5(3.4 \%)$ \\
Moderately healthy & $52(30.4 \%)$ & $109(63.7 \%)$ & $10(5.8 \%)$ \\
Unhealthy & $19(32.2 \%)$ & $12(20.3 \%)$ & $28(47.5 \%)$ \\
\hline
\end{tabular}

\section{Predictors of cluster transition}

The multinomial logistic regression analysis assessing the predictors of cluster transition showed that men were more likely than women to shift towards a healthier cluster compared to shifting towards an unhealthier cluster $(\mathrm{OR}=3.74,95 \% \mathrm{CI}=1.48-9.46, P=.009)$ and remaining in the same cluster $(\mathrm{OR}=1.96,95 \% \mathrm{CI}=1.04-3.68, P=.035)$ (see Table 6). Further, people who lived alone were more likely than people who lived together to be in the group that shifted towards an unhealthier cluster compared to the group who remained stable $(\mathrm{OR}=3.48,95 \% \mathrm{CI}=1.01-11.99, P=.045)$. 
Table 6. Predictors of cluster transition

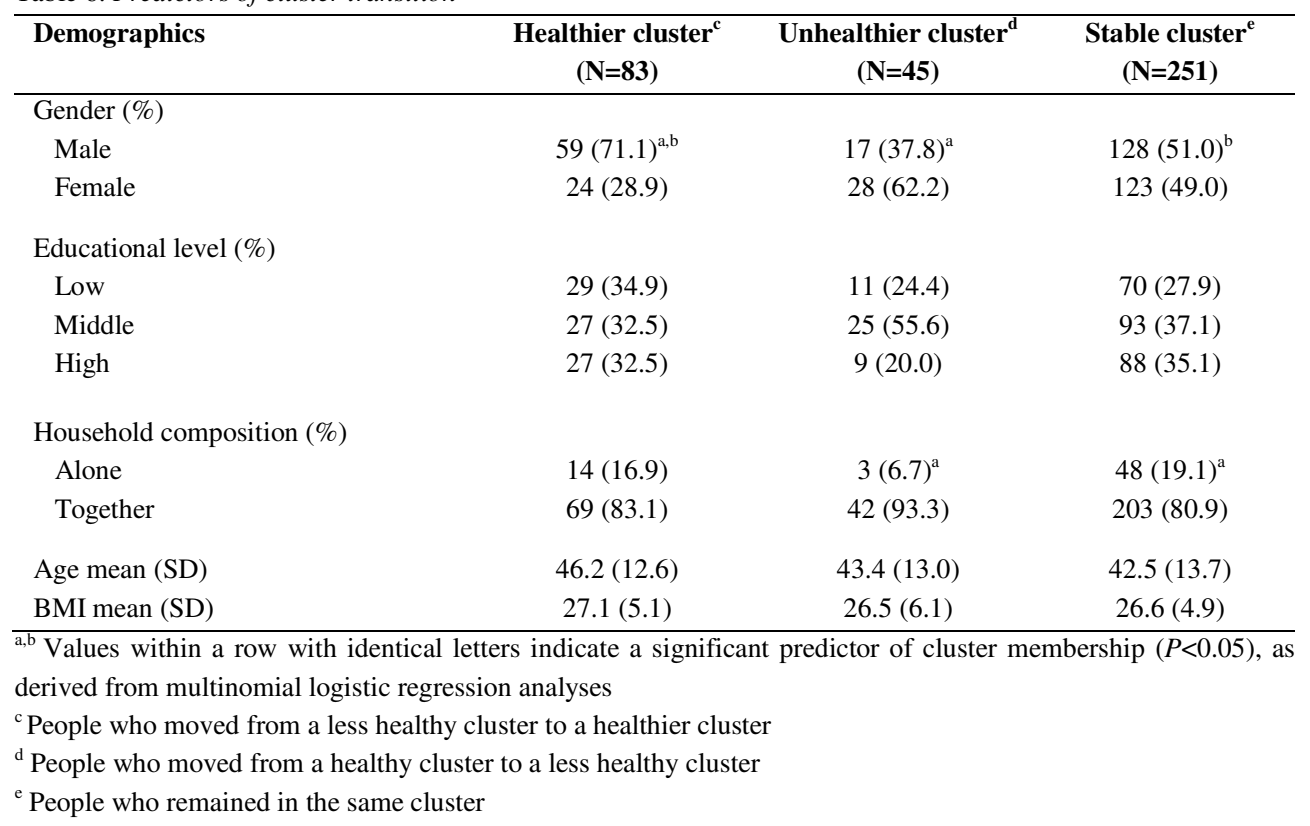

\section{Discussion}

The aim of this study was to determine whether or not it is useful to develop nutrition education intervention based on clusters of dietary patterns by examining the reproducibility of clusters and the stability of cluster membership at two time points in a six month period in a cohort of Dutch adults. Furthermore, sociodemographic determinants related to cluster membership and cluster transition were studied.

At both measurement points a comparable three-cluster solution was identified, distinguishing a healthy, moderately healthy, and unhealthy dietary pattern. As expected, the average daily intake of calories from energy-dense food products was lowest among people in the healthy cluster and highest in the unhealthy cluster (Bailey et al., 2006). In line with previous research, men and individuals with a low educational level were more likely to have a less healthy dietary pattern (Devlin et al., 2012; Greenwood et al., 2000; Martikainen, Brunner, \& Marmot, 2003; Newby \& Tucker, 2004). Our results further also show that about one third of our sample changed from dietary pattern during the study period, indicating that cluster membership is not stable. Most participants changed from a less healthy cluster to a healthier cluster. Men and people who lived alone were significantly more likely to shift towards a less healthy dietary pattern over time.

In order to be able to determine whether or not it is useful to develop nutrition education interventions based on clusters of dietary patterns, it is necessary to know if distinctive clusters of dietary patterns can be derived and reproduced over time, if cluster membership is stable, and if it is predictable which type of people belong to a certain cluster (Correa Leite et al., 2003; Devlin et al., 2012; Grieger et al., 2012; Gubbels et al., 
2013; Northstone et al., 2013). Our results show that the first of these three conditions is met. At both baseline and follow-up three distinctive clusters of dietary patterns were derived, which were also in line with previous research (Bailey et al., 2006; Crozier et al., 2006; Devlin et al., 2012; Newby \& Tucker, 2004) and the few longitudinal studies on this topic (Devlin et al., 2012; Hu et al., 1999). This finding adds to the scarce literature on this (Devlin et al., 2012) and implies that comparable and distinctive clusters of dietary patterns can be derived over time. It should, however, also be noted that although the classification of the baseline and follow-up clusters was comparable, the composition of the clusters was not $100 \%$ identical. There were, for example, a few differences in the composition among dairy products and sandwiches and fillings. This can be a consequence of filling out the baseline questionnaire, which can induce people to reflect on their behavior and lead to changes at follow-up. It is further also possible that participants changed spontaneously because they, for example, wanted to lose weight. However, although these differences in composition between the baseline and follow-up clusters are of importance, it can be concluded that the overall classification of the baseline and follow-up clusters was comparable, showing clusters of dietary patterns ranging from healthy to unhealthy.

Our results further also show that dietary patterns can change over time. While the majority of our study population remained in the same cluster between baseline and followup, about one third changed from cluster (i.e. dietary pattern). Although this change is lower than reported in a recent study among a cohort of 7 to 13 year old children (Northstone et al., 2013), this means that there is no fulfilment of the second condition (i.e. stability of cluster membership) since individuals' dietary patterns may change over time. This finding should, however, also be interpreted by taking into account the fact that cluster analysis is data driven. Any method of quantifying diet will result in some change over time and change of cluster can thus always be expected in longitudinal research. However, the size of change is important. Although the majority of our sample did not change from cluster of dietary pattern over time, it is debateable whether or not cluster analysis can be used as basis for nutrition education interventions. We, however, assume that cluster analysis is not suitable for this because using this approach would mean that one out of three would receive a misfitted (i.e. wrong) intervention.

In addition, in line with previous research our results also show that only few sociodemographic factors were associated with cluster membership (Newby \& Tucker, 2004; Northstone et al., 2013). Hence, it is difficult to predict in advance from certain characteristics if a person has an unhealthy, a moderately healthy, or a healthy dietary pattern. This makes it difficult to target interventions on high-risk groups with unhealthy dietary patterns. Only educational level and gender were significantly associated with one of the derived clusters. In addition, cluster transition was also merely predicted by two sociodemographic factors. These findings indicate that there also is no fulfilment of the third condition (i.e. predictability), which again implies that it may not be efficient to develop nutrition education interventions based on clusters of dietary patterns. 
The conclusion that clustering has only limited added value as a basis for intervention development should be interpreted by taking into account the limitations of our study. First, dietary intake was self-reported which may limit the validity of our study. However, to improve the reporting participants were provided with detailed information about, for example, the size of a standard portion (e.g. one glass of soda is equal to $200 \mathrm{ml}$ ). It should further be noted that the FFQ did not capture the whole diet. Possibly, the composition of the clusters would have been different if we had included additional food categories (e.g. breakfast cereals or fish). However, the aim of our FFQ was to obtain different types of dietary patterns based on energy-dense food products that are most common in the Dutch adult population. In addition, because the most common energydense food products were included, our FFQ was able to make a reliable distinction between healthy, moderately healthy, and unhealthy clusters. Second, a relatively short study period was used (i.e. 6 months) to examine the stability of food clusters and cluster membership. For future research, it is recommended to replicate our study with a longer follow-up period and examine the degree of cluster stability in more detail. It should further also be noted that participants belonging to the healthy cluster at baseline could only worsen (or remain stable), while participants in the unhealthy cluster could only improve (or remain stable). Finally, a limitation inherent to all research using cluster analysis is that this technique involves several subjective but important decisions, such as the grouping of foods, possible transformations of variables, choices about the method of clustering, labelling of the clusters, and the final number of clusters (Grafenauer et al., 2013; Grieger et al., 2012; Michels \& Schulze, 2005). It would, for example, also have been possible to use a two- or four- cluster solution. However, in-depth research showed that these alternative cluster solutions were inadequate (i.e. not interpretable and meaningless). By examining possible alternative cluster solutions, using a two-step clustering approach, and including three independent researchers during this process, we have tried to limit the subjectivity inherent to cluster analysis research as much as possible.

In contrast to these limitations, an important strength of this study is that it had a longitudinal design. Nearly all previous studies on dietary patterns have used crosssectional data to derive clusters of dietary patterns (Devlin et al., 2012; Grieger et al., 2012; Newby et al., 2003; Northstone et al., 2013; Togo et al., 2001). Further, thus far only one longitudinal study exists that has examined the stability of dietary patterns over time using cluster analysis (Northstone et al., 2013). This and the fact that we tried to identify the determinants of change of food cluster over time are important strengths of our study.

\section{Conclusions}

To conclude, because foods are consumed as part of an overall diet and not in isolation, it is suggested that focusing on clusters of dietary patterns can be a promising efficient strategy within nutrition education interventions (Correa Leite et al., 2003; Hearty \& Gibney, 2009, 2013; Millen et al., 2001; Millen et al., 2005; Newby et al., 2003; Newby \& Tucker, 2004; 
Togo et al., 2001). However, the use of clusters as basis for intervention development can only have added value when distinctive clusters of dietary patterns can be derived and reproduced over time, when cluster membership is stable over time, and when it is possible to predict who belongs to which cluster (Devlin et al., 2012; Gubbels et al., 2013; Northstone et al., 2013; Quatromoni et al., 2002). In our study we have examined these three aspects and showed that distinctive clusters of dietary patterns can indeed be derived and reproduced. However, about one third of our study population changed from one cluster to another over time and only few sociodemographic characteristics predicted cluster membership and cluster transition. Hence, it can be concluded that it may not be more efficient to target nutrition education interventions at clusters of dietary patterns. 


\section{PART 2}

\section{Intervention development and evaluation}




\section{CHAPTER 4}

Systematic development of a video and text version of a web-
based computer-tailored obesity prevention intervention

Published as: Walthouwer, M., Oenema, A., Soetens, K., Lechner, L., \& de Vries, H. (2013). Systematic development of a text-driven and a video-driven web-based computertailored obesity prevention intervention. BMC Public Health, 13(1), 978. 


\begin{abstract}
Background: This paper describes the systematic development of a text-driven and a video-driven web-based computer-tailored intervention aimed to prevent obesity among normal weight and overweight adults. We hypothesize that the video-driven intervention will be more effective and appealing for individuals with a low level of education.
\end{abstract}

Methods and design: The Intervention Mapping protocol was used to develop the interventions, which have exactly the same educational content but differ in the format in which the information is delivered. One intervention is fully text-based, while in the other intervention in addition to text-based feedback, the core messages are provided by means of videos. The aim of the interventions is to prevent weight gain or achieve modest weight loss by making small changes in dietary intake or physical activity. The content of the interventions is based on the I-Change Model and self-regulation theories and includes behavior change methods such as consciousness raising, tailored feedback on behavior and cognitions, goal setting, action and coping planning, and evaluation of goal pursuit. The interventions consist of six sessions. In the first two sessions, participants will set weight and behavioral change goals and form plans for specific actions to achieve the desired goals. In the remaining four sessions, participants' will evaluate their progress toward achievement of the behavioral and weight goals. They will also receive personalized feedback on how to deal with difficulties they may encounter, including the opportunity to make coping plans and the possibility to learn from experiences of others. The efficacy and appreciation of the interventions will be examined by means of a three-group randomized controlled trial using a waiting list control group. Measurements will take place at baseline and six and twelve months after baseline. Primary outcome measures are body mass index, physical activity, and dietary intake.

Discussion: The present paper provides insight into how web-based computer-tailored obesity prevention interventions consisting of self-regulation concepts and text-driven and video-driven messages can be developed systematically. The evaluation of the interventions will provide insight into their efficacy and will result in recommendations for future webbased computer-tailored interventions and the additional value of using video tailoring.

Keywords: obesity, physical activity, diet, intervention mapping protocol, computertailoring, weight management, education level 


\section{Background}

Obesity is a major global health problem (Finucane et al., 2011; Wang, McPherson, Marsh, Gortmaker, \& Brown, 2011). The World Health Organization estimated that in 2008 about 502 million adults worldwide were obese (Finucane et al., 2011) and it is expected that this figure will continue to rise in the future (Wang et al., 2011). An individual is classified as obese when the Body Mass Index (BMI: weight in kilograms / height in meters ${ }^{2}$ ) is 30 or higher (WHO, 2000). In the Netherlands, about $12 \%$ of the adult population is obese and it is expected that this proportion will rise to $18 \%$ by 2024 (Bemelmans, Hoogenveen, Visscher, Verschuren, \& Schuit, 2004). There is, however, a significant difference in obesity prevalence between individuals with a lower and a higher level of education (Devaux \& Sassi, 2011; Monteiro, Moura, Conde, \& Popkin, 2004; Uiters \& Verweij, 2012). In the Netherlands, $18.4 \%$ of individuals with a low level of education are obese, while this percentage is only $6.5 \%$ among people with a higher level of education, making the lower educated group an important target group for obesity prevention programs (Uiters $\&$ Verweij, 2012). These high and increasing prevalence rates are worrying, particularly because obesity is associated with a range of health problems, such as type 2 diabetes, cardiovascular diseases, musculoskeletal disorders, psychological disturbance, different types of cancer, and premature death (Dixon, 2010; Swinburn et al., 2004). Obesity also causes a considerable economic burden, due to health care use, absenteeism, disability payments, and loss of productivity (Dixon, 2010; Neovius et al., 2012; Swinburn et al., 2004; Wang et al., 2011). Because of the high burden of obesity, its prevention has become a high priority in many international and national public health policies (Bemelmans et al., 2004; Commision of the European Communities, 2007).

Nevertheless, effective and efficient interventions to prevent obesity that can reach large numbers of people are scarce (Gortmaker et al., 2011; Wing, 2003). Moreover, these interventions are not always specifically geared to meet the needs of individuals with a low level of education (Magnée et al., 2013; Monteiro et al., 2004; Uiters \& Verweij, 2012). Overall, it can be concluded that there is an urgent need for effective interventions to prevent obesity that can reach large numbers of people and that also appeal to and are effective for individuals with a lower level of education.

Ideally, obesity prevention interventions should have the possibility to adapt to an individual's characteristics, because in general each individual wants to manage his/her body weight in his/her own specific way (Kayman, Bruvold, \& Stern, 1990; Klem, Wing, McGuire, Seagle, \& Hill, 1997). Web-based computer-tailored interventions may fulfill this requirement since these interventions can provide personally adapted messages based on an individual's responses to, for example, an online questionnaire (de Vries \& Brug, 1999; Krebs et al., 2010). In the last decade, attention to web-based computer-tailored interventions has increased because of their potential advantages such as the ability to reach many individuals in a relatively cheap way (Cobiac, Vos, \& Barendregt, 2009) and the possibility for participants to use the intervention whenever they prefer (Oenema, Brug, 
Dijkstra, de Weerdt, \& de Vries, 2008). Further, as web-based computer-tailored interventions require minimal human contact, they are also potentially cost-effective (Oenema, Brug, \& Lechner, 2001). In addition, because the content of a web-based computer-tailored intervention can be adapted to an individual's characteristics, it is suggested that messages are better read, saved, and remembered as compared to nontailored materials (Brug et al., 2003). Several reviews have already reported the promising effects of web-based computer-tailored interventions for modifying dietary and physical activity behavior (Krebs et al., 2010; Kroeze, Werkman, \& Brug, 2006) and preventing weight gain (Wieland et al., 2012). Web-based computer-tailored interventions may, therefore, be a good innovation to help in targeting the public health problem of obesity.

We developed two versions of a web-based computer-tailored intervention aimed to prevent obesity among Dutch adults with a healthy weight or limited overweight (BMI between 18.5 and 30). Both interventions have exactly the same educational content, but differ in the format in which the information is delivered. One intervention is fully textbased, without the use of visual elements (text-text intervention), while the other provides the core messages by means of videos with additional text-based information (video-text intervention). Although tailoring is considered to be an effective health education technique, the way messages are communicated may be even more important. We hypothesize that the video-text intervention will be more appealing and effective for individuals with a low level of education. Lower educated individuals have, for example, lower literacy skills, making textual messages less effective for these individuals (PaascheOrlow et al., 2005). Providing messages by means of other communication formats, such as videos, may therefore be a better way to reach these individuals (Wilson et al., 2010). An advantage of video messages is the fact that these do not require abstract text to be translated into concrete actions (Petty \& Cacioppo, 1986a), which lower educated individuals often find harder to do (Cutler \& Lleras-Muney, 2010; Paasche-Orlow et al., 2005). Video messages reduce the cognitive effort needed to process information, which will free resources to process the main message and accordingly lead to a better understanding (Sweller, 1994). Research has already shown that videos can effectively attract attention and stimulate comprehension in lower educated individuals (Kathleen, 1997; Ma, Hua, Lu, \& Zhang, 2005; Wilson et al., 2010), while another study has shown a media preference in these individuals for video over text (Calvert, Rideout, Woolard, Barr, $\&$ Strouse, 2005). In addition, there is evidence that videos can be effective in improving cognitions such knowledge, attitude, self-efficacy, and intention (Krawczyk et al., 2012; J. Wang et al., 2012) as well as actual behavior (Armstrong et al., 2011; Wilson et al., 2012). Hence, video-text interventions may be a promising approach to effectively increase the attention and comprehension of individuals with a low level of education, which will facilitate information processing and ultimately behavior change (de Vries et al., 2003; McGuire, 1985). 
As the use of a planned approach during intervention development increases the likelihood for an intervention to be effective (Brug, Oenema, \& Ferreira, 2005), we used the Intervention Mapping (IM) protocol (Bartholomew et al., 2011) to develop the two versions of the intervention and the corresponding implementation and evaluation plan. The IM protocol is a framework for effective theory- and evidence-based decision making at each step in intervention development, implementation, and evaluation. The protocol consists of six succeeding steps that can be worked through in an iterative manner. The aim of this paper is to describe the systematic development of the two web-based computertailored interventions according to the six steps of the IM protocol.

\section{Methods \& design}

The interventions were developed by systematically going through the six steps of the IM protocol. In the first step of this protocol, a needs assessment is carried out using the PRECEDE model (Green \& Kreuter, 2005). The second step provides the foundation for the intervention by specifying in detail who and what will be changed through the intervention. In step three, theory-based methods and practical applications are identified that can be used to achieve the intervention goals. Step four concerns the actual program development, by using all the products of the previous steps; during this step, a pretest of the intervention is conducted as well. In step five, adoption and implementation plans are specified. Finally, in step six, both an effect and process evaluation plan are developed.

\section{Step 1: Needs assessment}

Obesity is caused by an energy imbalance, in which energy intake exceeds energy expenditure (van Kreijl, Knaap, \& van Raaij, 2006). Important obesity-related dietary behaviors associated with a high energy intake are consuming energy-dense food (i.e. high in fat, salt, and sugars), drinking sugared drinks and alcohol, and consuming large portions of foods (van Kreijl et al., 2006). Energy can be expended by means of physical activity, which can be divided in different sub-behaviors such as active transportation (e.g. cycling to work), daily activities (e.g. cleaning), leisure time activities (e.g. walking), and sports (Swinburn et al., 2004). Hence, physical activity and dietary intake are important targets in the prevention of obesity (Swinburn et al., 2004). By improving these two behaviors the energy balance can be restored, which in turn will prevent weight gain. In line with the fact that individuals with low levels of education have the highest rate of obesity, they also tend to have the highest energy intake (Giskes, Turrell, van Lenthe, Brug, \& Mackenbach, 2006) and the lowest physical activity levels (Mo, Turner, Krewski, \& Mo, 2005). This confirms the importance of developing interventions that are appealing and effective for these individuals.

Prevention of obesity is thought to be most successful when focusing on small changes in dietary intake and physical activity, since small changes are easier to initiate and maintain than large changes as strict diets or following an intensive sport schedule (Hill, 
2009). Further, achieving small changes could lead to an increased self-efficacy, which in turn could stimulate people to make additional small changes (Hill, 2009). This small changes approach assumes that changes of about 100 kilocalories (kcal) per day are sufficient to ensure an energy balance which is in equilibrium and accordingly prevent weight gain (Hill, 2009). This is equal to about 2,000 more steps of walking per day or 20 more minutes of other physical activities per day. To consume $100 \mathrm{kcal}$ less per day, individuals only have to consume a little less of energy-dense food or substitute food such as a regular soda for a diet soda or a snack for a piece of fruit. These kinds of moderate energy reductions are sufficient to result in sustained decreases in energy intake without increased feelings of hunger (Rolls et al., 2006). To lose a little weight (about five kilogram in one year), individuals have to make daily changes of $200 \mathrm{kcal}$.

Based on the small changes approach, the primary objective of the two web-based computer-tailored interventions is to realize small changes in physical activity and dietary intake of 100 or $200 \mathrm{kcal}$ per day in order to prevent obesity (i.e. maintain weight or achieve modest weight loss) among Dutch adults with a healthy weight or limited overweight.

\section{Step 2: Program objectives}

The goal of step two is to formulate change objectives, which are the most proximal goals of an intervention that help in achieving the behavioral and weight goals. The two components that are needed to formulate change objectives are performance objectives (i.e. sub-behaviors within the desired behavior) and their determinants (Bartholomew et al., 2011).

\section{Performance objectives}

It has been suggested that in particular self-regulation concepts are important to successfully achieve weight management goals, such as identification of a problem, setting goals, making plans, monitoring weight and behavior, and having coping skills (Brug, Oenema, \& Ferreira, 2005; de Ridder \& de Wit, 2006). Evidence has, for example, shown that individuals with adequate self-regulation skills are more successful in changing complex and habitual behaviors such as physical activity and dietary intake as well as in achieving weight maintenance (Chambers \& Swanson, 2012; Schnoll \& Zimmerman, 2001). The performance objectives (PO's) and accordingly the content of the interventions were, therefore, based on the concepts and phases of self-regulation models. For example, the first PO is 'individuals decide to prevent weight gain: maintain current weight or lose a little weight'. An overview of all PO's can be found in Table 1 (see appendix 1). It should be noted that although these PO's follow a sequence of action, it is possible to return to previous PO's. 


\section{Determinants}

The next step was to identify the most relevant (i.e. important and changeable) determinants of each PO. For this purpose we carried out an extensive literature review on determinant studies. During this process, the I-Change Model (de Vries et al., 2003) was used as framework to identify determinants for the pre-motivational, motivational, and postmotivational phase. This model is an integration of various behavior-oriented theories such as the Social Cognitive Theory, Health Belief Model, Theory of Planned Behavior, Transtheoretical Model, and Goal Setting theories. Based on a determinant analysis, the most relevant determinants that need to be targeted in the intervention are knowledge, awareness, risk perception, attitude, social influence, self-efficacy, intention, skills, and action planning (see Table 1 of appendix 1).

\section{Change objectives}

Change objectives (CO's) specify what individuals have to learn in order to accomplish PO's (Bartholomew et al., 2011). CO's are created by combining the PO's and determinants. In total, we formulated around $150 \mathrm{CO}$ 's. An example of a CO concerning PO 6 (i.e. make coping plans to deal with difficulties) and the determinant knowledge is 'individuals can explain how to deal with encountered difficulties'. Table 1 (see appendix 1) provides a selection of the most important CO's per PO and determinant.

\section{Step 3: Methods and applications}

In the third step, theoretical methods for changing each determinant were selected and translated to practical applications. Methods and applications were retrieved from literature concerning behavior change techniques and study protocols of previous studies (Abraham \& Michie, 2008; Bartholomew et al., 2011; Michie, Abraham, Whittington, McAteer, \& Gupta, 2009). Table 1 (see appendix 1) provides an overview of the most important theoretical methods and practical applications of the program. When applicable, this table also shows the differences in practical applications between the text-text and video-text intervention. The most important methods and applications are elucidated in more detail below. It should be noted that both interventions have exactly the same educational content in order to allow for a direct comparison between the text-text intervention and the videotext intervention. For the video-text intervention, particularly the practical applications related to PO 1, 2, 7, and 8 are converted into videos.

\section{Providing information}

To increase an individual's knowledge and skills, providing information is included as theoretical method. In line with the parameters of this method (e.g. information must be relevant) (Bartholomew et al., 2011), the program mainly consists of basic information that individuals are required to have in order to change their behavior successfully. Individuals will, for example, receive information about the small changes approach (PO 2). To prevent 
that participants have to read something that they already know or are not interested in, they mostly have the possibility to choose which information they want to read. The program, for example, consists of optional in-depth information, such as the amount of kcal in various food products, which can be read by clicking on hyperlinks. Important information, such as information about the small changes approach, is included in the regular sequence of the program, as this information is important for making further steps in the process. In both the video-text as text-text intervention, all information is provided by means of textbased messages.

\section{Consciousness raising and feedback}

Consciousness raising and feedback will be used to increase individuals' awareness about their body weight (PO 1 and 8), physical activity level, and dietary intake (PO 2, 7, and 9). For this purpose, individuals' have to answer various questions about, for example, their body weight and general dietary intake and physical activity pattern. Based on their answers, they will receive tailored feedback about how they can improve their weight and behavior. This feedback will help participants to set appropriate weight and behavior change goals. In the video-text intervention, all these messages will be delivered using videos. In these videos, the feedback is given by actors playing an expert in the field of nutrition and physical activity. Simultaneously with the video message, a table is shown below the videos consisting of very specific information, for example the different food products a participant consumes or a participant's BMI. In the text-text intervention, the same table will be provided only accompanied with text-based messages.

\section{Decisional balance}

To help participants decide which behavior change would suit them best (PO 2), a decisional balance format is included as well. For this purpose, individuals will be asked about their attitudinal, self-efficacy, and social influence beliefs regarding physical activity and dietary intake. Based on their answers, the program will suggest which behavior would be most suitable for them to change. A person who has indicated, for example, to have a positive attitude and sufficient self-efficacy expectations for a change in dietary intake will be advised to choose for a change in dietary behavior. Participants can decide whether or not they will work through this part of the program. This optional part will only be advised to individuals who indicate that they find it difficult to decide which behavior change would suit them best. Because this procedure is optional, this part is only elaborated by means of text and thus exactly the same in both versions of the intervention.

\section{Goal setting}

Goal setting is used as a method to help participants attain the desired behavior change (Latham \& Locke, 1991). In the program, participants can select a goal from a list with predefined options (i.e. goals) for weight, diet, and physical activity. We used a closed-ended 
goal setting format to prevent the formation of inadequate goals. Participants will be instructed about how to select a goal by means of written text. To further help participants set appropriate goals, they will receive extensive feedback on their behavior and weight, as mentioned before. After this feedback, participants have to decide whether they want to maintain their current weight or lose a little weight (PO 1) and how they want to achieve their weight goal, either by increasing their level of physical activity, improving dietary intake or both (PO 2). The possibility to change both behaviors is included, because several studies have shown that multiple health behavior interventions result in greater weight loss (Sweet \& Fortier, 2010), have higher participation rates (Robroek, van Lenthe, van Empelen, \& Burdorf, 2009), and may be more cost-effective (Sweet \& Fortier, 2010). Individuals subsequently have to choose specific sub-behavior(s) within the chosen main behavior. Within physical activity, individuals can choose from three sub-behaviors: active transportation, physical activity in leisure time, and sports. For dietary intake a distinction is made in: dairy produce, sandwiches, food at dinner, snacks, hot and cold beverages, and alcohol. Individuals can choose a maximum of two sub-behaviors per main behavior (i.e. dietary intake and physical activity). Finally, individuals also have to decide how they want to improve the chosen sub-behavior using again a pre-defined list with goals. For dietary intake, participants can choose to eat smaller portion sizes, consume a less energy-dense alternative or eat less of a certain food product. For example, an individual who chooses for snacks subsequently has to indicate whether he/she wants to eat fewer snacks, eat low energy-dense snacks or eat smaller portion sizes of snacks. For physical activity, individuals have to decide whether they want to increase the frequency or duration of activity. For example, an individual can decide to walk more times per week as well as to walk longer per time. As this whole goal setting application concerns choices and decisions individuals have to make, this part is exactly the same in both interventions and merely elaborated by means of text.

\section{Action, preparatory, and coping planning}

Research has shown that intended behavior changes are more likely to result in action when behavioral intentions are translated into concrete plans (Sheeran, Webb, \& Gollwitzer, 2005; Sniehotta, Scholzb, \& Schwarzerb, 2005). Hence, after individuals have set goals, they have to form plans for action to achieve the intended goals (PO 3). Action plans are generally made by means of the 'if-then' format (Sheeran et al., 2005). The 'if' specifies when a specific action has to be performed, while the 'then' describes what specific action will be taken. In the interventions, individuals have the opportunity to make three kinds of plans: action (i.e. plans to perform the specific behavior), preparatory (i.e. plans for preparation), and coping plans (i.e. plans to deal with difficult situations). Individuals first have to formulate one action plan for every chosen sub-behavior (e.g. if I have lunch break at work, then I will eat an apple instead of a candy bar). To realize the behavior change, individuals also have the opportunity to formulate a maximum of three preparatory plans 
for each chosen sub-behavior (e.g. If I go to the supermarket on Monday, I will buy five apples). Both the action and preparatory plans will be formulated using an open-ended 'ifthen' format. This format was chosen since a small pilot study among members of the target population has shown that this was the most preferred method for making action and preparatory plans. The 'if' and 'then' are predefined and individuals have to complete this sentence by filling out their own ideas and preferences. Individuals will also receive examples of good plans to help them formulate appropriate plans.

Individuals often encounter barriers that can hinder a successful behavior change (de Vries et al., 2003). Hence, a coping planning element will be introduced in the program once participants have started with the behavior change. They will be given the opportunity to make coping plans for situations in which they experience or expect difficulties to successfully perform the intended behavior. For each sub-behavior, individuals can specify a maximum of three coping plans using a closed-ended format, as the pilot study has also shown that this format was preferred for coping plans. Individuals can choose difficult situations from a pre-defined list. This list is composed based on findings from previous studies (Schulz et al., 2011; van Genugten et al., 2010) and a pilot study that was performed among members of the target population. To make a coping plan, individuals first have to choose which difficult situations they encountered or expect to encounter (e.g. a busy working day). Subsequently, they will receive feedback on how to deal with these situations followed by the opportunity to choose a coping response from a list for every chosen situation.

It should be noted that because of the large variety that can exist in plans and in order to provide clear examples of adequate plans, the planning part is carried out merely using textual messages. Hence, this part is exactly the same for the video-text and text-text intervention and there were no additional video messages.

\section{Modeling}

To target individuals' self-efficacy to carry out the desired behavior change and the corresponding plans (PO 6, 7, 8, and 10), modeling techniques are used. Narratives are used as a practical application for modeling. A narrative is a constructive format in which a sequence of events is described (Hinyard \& Kreuter, 2007). For each sub-behavior, two narratives are created. The narratives follow a chronological storyline in which a role model tells how his/her behavior change is going and how he/she deals with encountered difficulties. Each story consists of implicit tips and participants can choose whether they want to view these stories. In line with the parameters of modeling, all narratives show people who are struggling with their behavior change but eventually succeed in achieving their goal (Bartholomew et al., 2011). In the text-text intervention, the narratives merely consist of text, while the narratives in the video-text intervention are displayed by means of videos. In these videos an actor plays the role of a model (i.e. a person who also used the program). A different actor is used for each of the eighteen narratives in order to increase 
the credibility of the stories. To ensure that participants can identify themselves with the persons in the narratives, we used a variety of actors with differences in ethnic background, age, gender, educational level, and BMI. In the text-text intervention, these characteristics were described in text in the introduction of the narratives.

\section{Reinforcement and external attribution}

Besides modeling, participants' self-efficacy will also be targeted by means of reinforcement and external attribution. During the interventions, the progress toward enactment of plans and goal achievement of the participants will be evaluated. This evaluation will, for example, assess whether participants have gained, maintained, or lost weight (PO 7) as well as whether or not they have improved their physical activity level and/or dietary intake (PO 8). Participants who achieve their goals successfully will be complimented, while those who fail to achieve their goals will receive feedback in which the failure will be attributed to external factors that people cannot control in order to prevent a decrease in self-efficacy (Bandura, 1997). In the video-text intervention, this feedback will be provided by means of a video message, in which the progress of an individual is discussed. Simultaneously with the video message, a table is shown below the video consisting of an overview of participants' changes in body weight and dietary intake and/or physical activity during the program. In the text-text intervention, the same table will be provided, but then accompanied with text-based messages.

\section{Step 4: Program development}

\section{Scope and sequence}

The program consists of six sessions. Six sessions was considered to be sufficient to go through all phases of the I-Change Model and self-regulation theories. Figure 1 provides a flowchart of the six sessions, including a brief description of their content. In session one, participants have to decide whether they want to maintain their current weight or lose a little weight and how they want to achieve this goal: improving their physical activity level, dietary intake or both (PO 1 and 2). In session two, participants have to decide which specific sub-behaviors they want to change and how (PO 2), followed by the formation of action and preparatory plans (PO 3). It should be noted that the content of session one and two could have been combined into one large session, but because too much feedback may become too extensive for people to process (Kroeze et al., 2006), it was decided to use two separate sessions. However, at the end of session one, participants can decide to continue with session two directly. After session two, participants can start with enacting their plans and performing the desired behavior (PO 4 and 5). The remaining four sessions can be accessed in the next weeks, with at least one week between the sessions. The date for visiting the next sessions can be chosen by the participant. The focus of these sessions is on evaluating the progress toward meeting the weight and behavior change goals and enactment of the action plans and helping participants to deal with encountered or expected 
difficulties, for which they can make coping plans (PO 6, 7, and 8). In session four and five, participants have the possibility to change their goals and/or action plans (PO 9) and correspondingly go back to session two. In session four, five, and six, participants can further choose whether they want to view the narratives, which can help them in generating ideas for coping plans. Finally, the sixth session gives additional attention to how to maintain the behavior change on the long-term (PO 10). In total, participants can use the assigned intervention for a period of maximum three months, which is sufficient to follow all sessions. Each session lasts approximately 15 minutes, even though session two (in which current behavior is assessed) may take a bit longer to complete.

\section{Development}

The two interventions are developed using the Tailorbuilder software (OSE, the Netherlands), a program which is specifically designed to develop web-based computertailored interventions. To enhance participant retention and outcomes (Brug, Oenema, Kroeze, \& Raat, 2005), the interventions are integrated into a website (http://www.gewichtin-balans.info). This website provides information about the study, the intervention, and answers to frequently asked questions. Participants have to log in to the website in order to use their assigned intervention. Once logged in, participants also have the opportunity to read information and advices from previous sessions.

For the video-text intervention 240 videos were recorded. Because of budgetary constraints it was unfortunately not possible to convert all educational messages into videos. In all video messages an actor reads the messages aloud (i.e. the text in the text-text intervention). The video messages related to physical activity were recorded in a gym and given by a professional male actor, while the messages related to dietary intake were recorded in a cooking studio using a professional female actor. These two actors had the role as an expert in the field of nutrition and physical activity. For the narratives, two actors per sub-behavior were recruited, who each had to act in three videos. These videos were recorded in a film studio that was decorated as a living room in order to increase the authenticity of the stories. An example of the text-text intervention and the video-text intervention can be found in appendix 2 and 3 .

\section{Pretest of the interventions}

Two pretests were conducted before a definite version of the interventions was developed. First, a prototype of the text-text intervention was pretested among 25 members of the target population. Twenty individuals worked through the intervention at home, while five participants participated in a cognitive interview in the presence of a member of the project team. During this interview, participants were asked to talk out loud about the choices they made in the program and about their interpretation of the feedback, usability of the action planning tools and so on. This process yielded useful findings, for example about log in problems and unclear questions and feedback. Based on these findings, the text-text 
intervention was improved after which the videos were recorded. Subsequently, a pretest of the video-text intervention was conducted using the same procedure as the first pretest. This second pretest, however, focused more on the clarity of the video messages as well as the usability of the website in which the interventions are integrated. This pretest again resulted in several useful suggestions for improvement, such as speed problems during the program and difficulties with navigation through the website.

\section{Step 5: Planning for program implementation}

The purpose of step five is to develop a plan for program use, including adoption, implementation, and continuation. The need to facilitate program use was, however, already taken into account from the beginning of step one by developing the interventions in such a way that will enhance their potential for being adopted, implemented, and sustained. For example, to implement the interventions no human actions are required. Individuals who want to use the intervention can go the website and register, after which they will automatically receive programmed e-mails. Furthermore, the development process of the interventions may also increase the likelihood of program use. For example, several small pilot studies among members of the target population were conducted to identify the preferences and needs of the potential users regarding the content of the intervention, the visual aspects of the website and intervention, the format of making preparatory, action, and coping plans, the recruitment materials for the evaluation study (e.g. brochure), the included difficult situations, the name of the intervention, and the actors in the video messages. During these pilot studies, we particularly investigated the opinions of individuals with a low level of education to ensure their preferences are integrated in the intervention and all intervention parts were clear to them. Moreover, by pretesting the texttext intervention and subsequently the video-text intervention, we also tried to adapt the intervention as much as possible to users' preferences and needs.

To further facilitate program use, we also composed an advisory board consisting of potential adopters and implementers, including several occupational health services. Occupational health services have the objective to provide both cure and care for working people, which is in line with the purpose of the intervention. In the Netherlands, many employees receive an annual medical screening carried out by an occupational health service (RIVM, 2012). During these medical screenings, employees can be handed over a brochure about the intervention. Other implementation possibilities could be the use of advertisements in mass media, social media, and newsletters in companies. 
Figure 1. Overview of the content of the intervention

\begin{tabular}{|c|c|}
\hline & Session 1 \\
\hline Generic inf & rmation about aim and content of the program \\
\hline $\begin{array}{l}\text { Assessmen } \\
\text { towards we }\end{array}$ & $\begin{array}{l}\text { of demographic characteristics and questions concerning risk perception } \\
\text { ight gain }\end{array}$ \\
\hline $\begin{array}{l}\text { Feedback } 0 \\
\text { towards w }\end{array}$ & $\begin{array}{l}\text { n participants' BMI and their perceived severity and susceptibility beliefs } \\
\text { ight gain }\end{array}$ \\
\hline Generic inf & rmation about small changes approach and body weight \\
\hline Optional & Information about required changes to maintain weight or lose a little weight \\
\hline $\begin{array}{l}\text { Goal settin } \\
\text { weight loss }\end{array}$ & $\begin{array}{l}\text { : participants have to select a weight goal: weight maintenance or modest } \\
(5 \mathrm{~kg} \text { in one year) }\end{array}$ \\
\hline Short asses & ment of physical acitivity pattern and dietary intake \\
\hline $\begin{array}{l}\text { Feedback o } \\
\text { participant }\end{array}$ & $\begin{array}{l}\text { behaviors: it will be indicated which behavior change would fit the } \\
\text { best }\end{array}$ \\
\hline $\begin{array}{l}\text { Participan } \\
\text { are most } n\end{array}$ & $\begin{array}{l}\text { are asked if they want more information about for which behavior change they } \\
\text { otivated }\end{array}$ \\
\hline Optional & $\begin{array}{l}\text { Assessment of motivation: attitude, self-efficacy, and social influence beliefs } \\
\text { towards physical activity and dietary intake }\end{array}$ \\
\hline & $\begin{array}{l}\text { Feedback on beliefs and it will be indicated which behavior change fits best } \\
\text { with participants' motivation }\end{array}$ \\
\hline $\begin{array}{l}\text { Goal settin } \\
\text { dietary inta }\end{array}$ & $\begin{array}{l}\text { articipants have to select a weight goal: improve physical activity level, } \\
\text { or both }\end{array}$ \\
\hline Summary 0 & goals and overview of session 2 \\
\hline Choose dat & of next session: continue directly, after one week or choose own date \\
\hline & Session 2 \\
\hline Summary 0 & goals set in session 1 and overview of content session 2 \\
\hline Assessmen & of weight \\
\hline $\begin{array}{l}\text { Detailed as } \\
\text { intake or b }\end{array}$ & $\begin{array}{l}\text { essment of the chosen behavior change in session } 1 \text { (physical activity, dietary } \\
\text { th) }\end{array}$ \\
\hline $\begin{array}{l}\text { Feedback o } \\
\text { achieve the }\end{array}$ & $\begin{array}{l}\text { n behavior: it will be indicated which sub-behavior changes can be made to } \\
\text { weight goal }\end{array}$ \\
\hline $\begin{array}{l}\text { Goal settin } \\
\text { main behav }\end{array}$ & $\begin{array}{l}\text { : participants have to select specific sub-behavior change(s) within the chosen } \\
\text { ior (i.e. dietary intake and/or physical activity) }\end{array}$ \\
\hline Summary o & goals and information about planning \\
\hline $\begin{array}{l}\text { Action plan } \\
\text { specifying }\end{array}$ & $\begin{array}{l}\text { ng: for each sub-behavior participants have to form 'if-then' plans by } \\
\text { len, where, and how they want to carry out the desired behavior change }\end{array}$ \\
\hline Optional & $\begin{array}{l}\text { Preparatory planning: participants can form 'if-then' plans for preparations } \\
\text { that have to be made before the behavior change can be carried out }\end{array}$ \\
\hline Summary o & goals and action and preparatory plans \\
\hline Optional & Information on how to best carry out action and preparatory plans \\
\hline Generic inf & rmation about setting rewards \\
\hline Optional & $\begin{array}{l}\text { Setting rewards: participants can specify a future reward for when achieving } \\
\text { their weight goal }\end{array}$ \\
\hline $\begin{array}{l}\text { Summary } 0 \\
\text { Participant }\end{array}$ & $\begin{array}{l}\text { goals and action and preparatory plans and overview of session } 3 . \\
\text { will be instructed and motivated to carry out the behavior change }\end{array}$ \\
\hline Choose dat & of next session: after one week or choose own date \\
\hline
\end{tabular}

\begin{tabular}{|c|c|}
\hline \multicolumn{2}{|r|}{ Start behavior change } \\
\hline \multicolumn{2}{|r|}{$\downarrow$} \\
\hline \multicolumn{2}{|r|}{ Session 3} \\
\hline \multicolumn{2}{|c|}{ Summary of goals and action plan(s) and overview of content session 3} \\
\hline \multicolumn{2}{|c|}{ Assessment of weight } \\
\hline \multicolumn{2}{|c|}{ Assessment of the chosen sub-behavior(s) in session 2} \\
\hline \multicolumn{2}{|c|}{$\begin{array}{l}\text { Assessment of action plan(s) made in session } 2 \text { by asking participants when they failed to } \\
\text { carry out their plan(s) }\end{array}$} \\
\hline \multicolumn{2}{|c|}{$\begin{array}{l}\text { Feedback on sub-behavior(s): it will be indicated whether or not participants achieved } \\
\text { their behavioral goal by comparing their current behavior with their behavior in session } 2\end{array}$} \\
\hline \multicolumn{2}{|c|}{$\begin{array}{l}\text { Feedback on action plan(s): it will be indicated whether or not participants succeeded to } \\
\text { carry out their action plan(s) successfully }\end{array}$} \\
\hline \multicolumn{2}{|c|}{$\begin{array}{l}\text { Assessment of difficult situations: participants are asked if they encountered or expect to } \\
\text { encounter situations in which they find it difficult to carry out their action plan(s) }\end{array}$} \\
\hline \multirow{4}{*}{$\begin{array}{l}\text { Optional: } \\
\text { coping } \\
\text { planning }\end{array}$} & $\begin{array}{l}\text { Assessment of difficult situations: per sub-behavior participants can select a } \\
\text { maximum of two situations in which they experienced or expected to } \\
\text { experience difficulties in carrying out their action plan(s) }\end{array}$ \\
\hline & Feedback on how to deal with the selected difficult situation(s) \\
\hline & $\begin{array}{l}\text { Coping planning: participants can choose a coping option for how to deal with } \\
\text { the selected difficult situation(s) }\end{array}$ \\
\hline & Overview of coping plan(s) \\
\hline Optional & Generic information on how to deal with difficult situations in general \\
\hline \multicolumn{2}{|c|}{ Summary of goals and action and coping plans and overview of session 4} \\
\hline \multicolumn{2}{|c|}{ Choose date of next session: after one week or choose own date } \\
\hline
\end{tabular}

Choose date of next session: after one week or choose own date

$$
\text { Session } 4
$$

This session is idential to session 3 , only with the addition of:

After the feedback on sub-behavior(s) and action plan(s): participants have the possibility to change their goals and/or action plan(s). Participants who want to change their goals and/or action plan(s) will go back to session 2 and follow the program again with their new goals and/or new action plan(s)

Optional: $\quad$ After the optional coping planning part, participants are asked if they want to after $\mathrm{read} / \mathrm{view}$ narratives

coping

planning Narratives: per sub-behavior participants can read/view two narratives

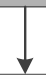

Session 5

This session is idential to session 4 , only with the addition of:

After the assessment of weight, behavior, and action plans, participants will for the first time receive feedback on their body weight during the program. It will be indicated whether or not they achieved their weight goal

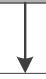

Session 6

This session is idential to session 5 , only with the addition of a part related to long-term changes at the end of this last session:

Generic information about how to maintain weight (changes) on the long-term

Goal setting: participants have to set a long-term weight goal by selecting a time-frame and desired future body weight

Summary of long-term weight goal and generic information about how to maintain behavior changes on the long-term

Optional Long-term action plan(s): participants can make new action plan(s) for how to maintain the behavior change on long-term

Summary of goals and action plan(s)

Optional Recapitulation of the essentials elements of the program

Official ending of the program 


\section{Step 6: Planning for program evaluation}

\section{Evaluation design and procedure}

A three-group randomized controlled trial will be conducted to evaluate the efficacy of both versions of the intervention in comparison to a waiting list control group on BMI, dietary intake, and physical activity. Participants who will be randomized to the control group can use one of the interventions after the study. Measurements will take place at baseline (T0) and at 6 (T2) and 12 months (T3) after baseline. During all measurements, participants will have to complete an online questionnaire via the study website.

A power calculation was carried out to estimate the required number of participants. In order to detect a medium sized effect (d=0.5) on BMI (Cohen, 1992), using a power of .90 , a significance level of .05 , and being able to test interaction effects between participants with a low, medium, and high level of education, about 1,000 participants need to complete the study. Taking into account a drop-out percentage of $50 \%$ between baseline and the last follow-up measurement (Eysenbach, 2005), this implies that approximately 2,000 participants are needed at baseline. These will be divided over the three study arms equally. Participants are eligible for inclusion when they are at least 18 years old, have a job, and have a BMI between 18.5 and 30. Although obese people (BMI $\geq 30)$ do not belong to the target population for the evaluation study, these individuals will be given the possibility to use the program. Participants who have a physical condition that may influence dietary or physical activity patterns (e.g. diabetes) are ineligible to participate. Participants will be recruited by various occupational health services during medical screenings as well as through companies without interference of the occupational health services. Brochures and messages in internal company newsletters will be used to invite people to participate in the study. Mass media advertisements and press releases will also be used to recruit participants. After registration at the study website, participants will be randomized to one of the three conditions and have the opportunity to fill out the baseline questionnaire. Participants in one of the intervention conditions have to wait two weeks before they can start using their assigned intervention. To decrease the likelihood of attrition in the evaluation study, one hundred cash prizes of $€ 100$ will be raffled among participants who complete all questionnaires (Robroek et al., 2009). Participants will also receive two reminders per intervention session and per measurement in order to improve retention in the study.

The Ethical Commission of the Open University Heerlen reviewed the study protocol and judged that no approval of the medical ethics committee was required. They also indicated that there was no objection to performance of the study. The study is registered in the Dutch Trial Registry (nr. NTR3501). Participants' approval will be obtained in line with the APA informed consent ethical principles. At the beginning of the study, all eligible participants will be provided with information about the study and asked to sign an online informed consent form. 


\section{Hypotheses}

The following hypotheses will be tested:

- The video-text intervention will be more effective than the text-text intervention in the prevention of weight gain (i.e. achieving weight maintenance or modest weight loss) and more appealing for individuals with low levels of education.

- The text-text intervention will be more effective than the video-text intervention in the prevention of weight gain and more appealing for individuals with high levels of education.

- Both the video-text as the text-text intervention will be more effective in the prevention of weight gain compared to the control group.

\section{Measurement instruments}

The primary outcomes of the study are self-reported body weight, BMI, physical activity, and dietary intake. These will be assessed at baseline and at 6 and 12 month follow-up. In a sample of the participants, body weight and height will also be measured objectively by various occupational health services at baseline and 12 month follow-up.

Further, several potential mediators and moderators of the intervention effects will be measured. Mediators (i.e. working mechanisms of the interventions) that will be assessed include self-regulation skills and self-efficacy and intention towards being more physically active and eating less of energy-dense food products. These will be measured at baseline and at 6 and 12 month follow-up. At baseline, various moderators will be measured as well, including demographic characteristics (e.g. gender, age, and educational level), information processing style, self-efficacy, intention, media preference, and perceived body image.

In addition, we will also conduct a process evaluation to examine potential moderating factors. This evaluation will take place directly after the last session of the intervention (T1) as well as briefly at the 6 month follow-up measurement (T2). Process measures that will be examined include action planning (i.e. the quality of action plans), intervention use (e.g. number of times participants logged in and amount of information viewed), and participants' appreciation of the intervention (e.g. source, message, and channel factors).

Figure 2 gives an overview of the concepts that will be measured at each time point. 


\section{Moderators:}

- Demographic characteristics (T0, T2, T3)

- Information processing style (T0, T2, T3)

- Self-efficacy (T0, T2, T3)

- Intention (T0, T2, T3)

- Program appreciation (T1, T2)

- Action planning (T1)

- Intervention use (T1)

- Media preference (T0)

- Perceived body image (T0)

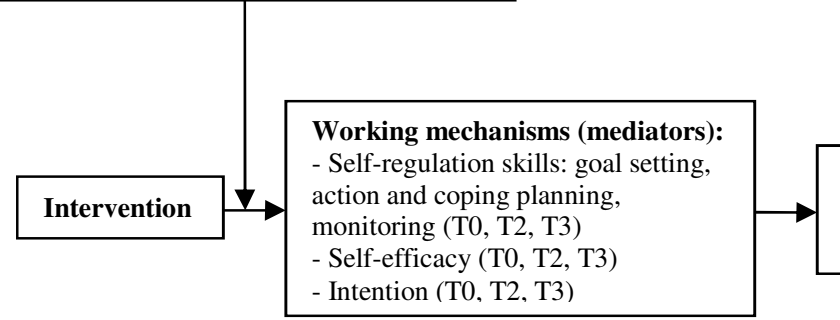

\section{Behavioral outcomes:}

- Physical activity (T0, T2, T3)

- Dietary intake (T0, T2, T3)
Health outcomes:

- Body weight and

BMI (T0, T2, T3)

Measurement times: T0 = baseline; T1 = after intervention session 6; T2 = 6-month follow-up; T3 = 12-month follow-up

\section{Discussion}

The aim of the present paper was to describe the systematic development of a text-driven and a video-driven web-based computer-tailored intervention aimed to prevent obesity among lower and higher educated Dutch adults with a healthy weight or limited overweight. Both interventions have exactly the same educational content but differ in the format in which the information is delivered. The video-text intervention provides the core messages by means of videos, while the text-text intervention is fully text-based without the use of visual elements. Both interventions follow the steps distinguished in the I-Change Model and self-regulation theories; individuals will be made aware of their health risk behavior, motivated to improve their dietary or physical activity behavior, set goals, make plans, monitor and evaluate their weight and behavior, and deal with difficult situations in order to achieve weight maintenance or modest weight loss.

The video-text intervention is specifically developed to respond to the need to develop interventions aimed at individuals with a low level of education. Despite the fact that these individuals have the highest rate of obesity (Monteiro et al., 2004; Uiters \& Verweij, 2012) and unhealthy dietary and physical activity patterns (Giskes et al., 2006; Mo et al., 2005), there is a lack of interventions that are appealing and effective for this high risk group. The video-text intervention may be a good way to reach these individuals, as research has shown that lower educated individuals have a preference for videos over text and evaluate video messages as more attractive and understandable than text (Calvert et al., 2005; Kathleen, 1997; Ma et al., 2005; Wilson et al., 2010). A related advantage of the video-text intervention is the fact that videos may reduce the amount of cognition needed to process information, which will free resources to process the main message (Sweller, 
1994). By evaluating the interventions, insight will be obtained into whether the video-text intervention is indeed more effective and appealing for lower educated individuals than the text-text intervention.

Components that may increase the efficacy of both interventions in general are the self-regulation framework and the small changes approach. Evidence indicates that these are promising ways to prevent obesity (Chambers \& Swanson, 2012; de Ridder \& de Wit, 2006; Schnoll \& Zimmerman, 2001). The fact that we used a planned approach to develop the interventions (i.e. the IM protocol) may also increase the overall efficacy (Brug, Oenema, \& Ferreira, 2005). Moreover, by adapting the interventions to potential users as much as possible, by integrating their preferences as indicated in several small pilot studies, the efficacy of the interventions may also be facilitated. Additionally, the interventions offer a lot of freedom. Participants can, for example, choose which goals they want to achieve and which information and advices they do or do not want to read. It has been assumed that this high sense of autonomy will enhance individuals' motivation, which in turn may increase the efficacy of the interventions (Ryan \& Deci, 2000).

To conclude, the use of the IM protocol has led to the development of two webbased computer-tailored interventions aimed to prevent obesity among Dutch adults with a healthy weight or limited overweight. By evaluating the interventions more insight will be obtained about their efficacy to prevent obesity, the efficacy of videos in web-based computer-tailored interventions, for which target group video tailoring is more effective, and by which processes effects may occur. These results can be used as input for future web-based computer-tailored interventions, specifically for those aimed at obesity prevention. If the interventions prove to be effective, an efficient obesity prevention tool that can reach large numbers of people against low costs will be available. 


\title{
CHAPTER 5
}

\begin{abstract}
Are there differences in effects and appreciation between a video and text version of a web-based computer-tailored obesity prevention intervention?
\end{abstract}

Accepted as: Walthouwer, M., Oenema, A., Lechner, L., \& de Vries, H. Are there differences in effects and appreciation between a video and text web-based computertailored obesity prevention intervention? A randomized controlled trial. Journal of Medical Internet Research. 


\begin{abstract}
Background: Web-based computer-tailored interventions have the potential to improve health behaviors in a cost-effective way. Yet, they often suffer from small effect sizes and high drop-out, in particular among people with a low educational level. Using videos as delivery format can possibly improve the effects and attractiveness of these interventions.
\end{abstract}

Objective: The main aim of this study was to evaluate the effects of a video and text version of a web-based computer-tailored obesity prevention intervention in comparison to a waiting list control condition on dietary intake, physical activity level, and BMI among Dutch adults. A related aim was to examine educational differences in efficacy of the two versions of the intervention. The final study aim was to evaluate differences in appreciation between the video and text version, including possible differences per educational level as well.

Methods: A three-armed randomized controlled trial was conducted with a baseline and 6 months follow-up measurement. The intervention was developed using the Intervention Mapping protocol and was based on self-regulation theories and the I-Change Model. The intervention consisted of six sessions, which each lasted about 15 minutes. In the video version, the core tailored information was provided by means of videos. In the text version the same tailored information was provided in text format. Outcome variables were selfreported and included BMI, physical activity, energy intake, and appreciation of the intervention. Multiple imputation was used to replace missing values. The effect analyses were carried out with multiple linear regression analyses and adjusted for confounders. The process evaluation data were analyzed with independent samples t-tests.

Results: The baseline questionnaire was completed by 1,419 participants and the 6-month follow-up measurement by 1,015 participants (71.5\%). No significant interaction effects of educational level were found on any of the outcome variables. Compared to the control condition, the video version resulted in lower BMI $(B=-0.25, P=.049)$ and lower average daily energy intake from energy-dense food products $(B=-175.58, P=.000)$, while the text version only had an effect on the latter one $(B=-163.05, P=.001)$. No effects on physical activity were found. The video version was rated significantly better than the text version on feelings of relatedness $(P=.041)$, usefulness $(P=.047)$, and grade given to the intervention $(P=.018)$.

Conclusions: The video version of the web-based computer-tailored obesity prevention intervention was the most effective intervention and appreciated best. Future research is needed to examine if it is cost-effective to use videos as delivery format of tailored health information in web-based computer-tailored obesity prevention interventions.

Keywords: RCT, web-based, computer-tailoring, obesity, educational level, delivery strategy 


\section{Introduction}

Overweight and obesity rates have increased rapidly during the last 30 years worldwide (Stevens et al., 2012; WHO, 2014b). In 2008, around 900 million adults were overweight and 502 million were obese (Finucane et al., 2011; WHO, 2014a). Among the Dutch adult population in 2012 , $48 \%$ was overweight whereas about $12 \%$ was obese (Bemelmans et al., 2004; van Bakel \& Zantinge, 2014). In many western countries, these figures are significantly higher among people with a low educational level (Devaux \& Sassi, 2011; McLaren, 2007; Roskam et al., 2010). For example, in the Netherlands the prevalence of overweight is $64.4 \%$ among adults with a low educational level compared to $40.1 \%$ among adults with a high educational level (Uiters \& Verweij, 2012).

To prevent weight gain, it is important to achieve a balance between calories consumed and calories expended (WHO, 2014a). This can be realized in many different ways. Recently, it has been suggested that weight management interventions can be best focused on small changes in energy intake and expenditure of about 100 or 200 kilocalories (kcal) per day (Hill, 2009). Such changes are sufficient to prevent weight gain (100 kcal) or achieve modest weight loss $(200 \mathrm{kcal})$ and are furthermore easier to initiate and maintain than aiming to achieve substantial weight loss, which requires strict diets or following an intensive sport schedule (Hill, 2009). Hence, weight management interventions should try to inform and help people how they can make such small changes and maintain them on the long-term.

Because overweight and obesity affect large numbers of people, these interventions should have the possibility to reach many people in an efficacious yet costeffective manner (Wieland et al., 2012). Furthermore, it is also important that these interventions have the possibility to adapt the information to an individual's unique behavior, preferences, and characteristics since in general each individual has his or her own preference about how he or she wants to improve their dietary intake and physical activity pattern (Kayman et al., 1990; Klem et al., 1997). Web-based computer-tailored interventions meet all these requirements, since these interventions aim to change people's health behavior by providing individually adapted information via the Internet (Kroeze et al., 2006). These interventions have the important advantage that they can be disseminated easily among a large target population against relatively low costs (Wieland et al., 2012). Research has already shown that web-based computer-tailored interventions can be costeffective in the promotion of various health behaviors (Schulz, Smit, et al., 2014; Smit, Evers, de Vries, \& Hoving, 2013). Another advantage is that web-based interventions can be used in privacy and at a time and place participants find convenient. Moreover, people are increasingly using the Internet as source for searching health-related information (Al Ghamdi \& Moussa, 2012; Beck et al., 2014; Internet World Stats, 2013; Siliquini et al., 2011), which further supports the choice for use of a web-based intervention.

Several reviews have demonstrated that web-based computer-tailored interventions can have positive effects on physical activity, dietary intake, short-term 
weight loss, and weight maintenance (Broekhuizen et al., 2012; Kroeze et al., 2006; Neville et al., 2009a; Wieland et al., 2012), even though there are also reviews that have concluded that the effectiveness for these interventions is inconclusive (Enwald \& Huotari, 2010; Neville et al., 2009b). All reviews conclude that effect sizes are mostly small and effects are mostly found at the short-term. Moreover, web-based computer-tailored interventions often suffer from high drop-out rates that reach up to around 50\% (Bennett \& Glasgow, 2009; Evers, Cummins, Prochaska, \& Prochaska, 2005; Eysenbach, 2005; Soetens et al., 2014). Web-based computer-tailored interventions have in particular problems with effectively reaching people with a low educational level (Magnée et al., 2013), while these people are most in need of change, given the higher prevalence of overweight and obesity. Hence, to optimize the potential of web-based computer-tailored obesity prevention interventions, it is necessary to examine how their impact and attractiveness can be improved, in particular for people with a low level of education (Magnée et al., 2013; van Genugten, van Empelen, Boon, et al., 2012; Walthouwer, Oenema, Soetens, Lechner, \& de Vries, 2013).

One possible solution may be to provide the information within these interventions by means of a delivery format that better fits the receivers' preferences (Brouwer et al., 2011; Wilson et al., 2010). All previous web-based computer-tailored weight management interventions have primarily used text-driven messages to provide information. However, people with a low educational level generally are less text-oriented and this could explain why the use and effectiveness are lower among these people (Paasche-Orlow et al., 2005). Recent studies, for example, highlight the potential of using videos as means to improve the use and effectiveness of web-based computer-tailored interventions among lower educated people in particular (Stanczyk et al., 2011; Walthouwer et al., 2013). Possibly, video messages are more appropriate for lower educated people because abstract text then does not have to be translated into concrete actions (Petty \& Cacioppo, 1986a), something that lower educated individuals often find harder to do (Cutler \& Lleras-Muney, 2010; PaascheOrlow et al., 2005). This will reduce the cognitive effort needed to process information and will free resources to process the main message and accordingly lead to a better understanding (Sweller, 1994).

To examine if the use of videos can indeed improve the effectiveness and attractiveness, we developed two versions of a web-based computer-tailored intervention aimed to achieve small changes in dietary intake and physical activity in order to prevent weight gain among Dutch adults with a healthy weight or with overweight (BMI between 18.5 and 30). Both versions had exactly the same content, but a different information delivery format. One intervention was fully text-based, without the use of visual elements (text version), while the other provided the core tailored information by means of videos (video version). The main aim of this study is to evaluate the effects of these two versions of the intervention in comparison to a waiting list control condition on dietary intake, physical activity level, and BMI among Dutch adults at 6 months follow-up. A related aim 
is to examine educational differences in efficacy of the two versions of the intervention. The final study aim is to examine differences in appreciation between the video and text version, including possible differences per educational level. We hypothesize that the video version will be more effective and appreciated better among lower educated participants.

\section{Methods}

The Ethical Committee of the Open University Heerlen reviewed the study protocol and decided that there was no objection to performance of the study. The study is registered in the Dutch Trial Register (NTR3501).

\section{Study design and respondents}

A three-armed randomized controlled trial was conducted with two experimental conditions (video and text intervention) and a waiting list control group that had the opportunity to use one of the interventions after the study. Measurements took place at baseline (T0) and 6 months (T1) after baseline. Criteria for participation were: being at least 18 years old, having a paid job (because of initial recruitment procedure), a BMI between 18.5 and 30, and sufficient command of the Dutch language. People with a physical condition that severely influenced their dietary or physical activity pattern (e.g. diabetes) were ineligible to participate.

It was estimated that 2,000 participants were needed to complete the baseline questionnaire in order to be able to detect a medium sized effect $(\mathrm{d}=0.5)$ on BMI and behavior with a power of .90 , a significance level of .05 , and taking into account a drop-out percentage of $50 \%$ between baseline and follow-up. This number of participants would also allow testing interaction effects between participants with a low, medium, and high level of education (Walthouwer et al., 2013).

\section{Procedure}

Participants were recruited from September 2012 until February 2013. Participants were initially recruited by handing out brochures during routine medical screenings by various occupational health centers. Unfortunately, this main recruitment strategy resulted in only few participants and additional recruitment strategies were necessary. Therefore, we also recruited participants directly through companies without interference of occupational health centers. An email was send to employees of the worksites that were willing to participate to inform them about the study. In addition, advertisements in national and local newspapers were also used to recruit participants. All recruitment materials (i.e. brochures, emails, advertisements) included information about the intervention and the study as well as a hyperlink of the study website where participants could register to participate. After registration and giving online informed consent, participants were randomly assigned to one of the three study conditions in a computer determined sequence. After randomization they received a username and password by e-mail that gave them access to the online 
baseline questionnaire. Two weeks after completion of this questionnaire, participants in the intervention conditions were given access to the intervention. Participants could use the assigned intervention for a maximum period of three months. Six months after baseline participants were asked by email to fill out the online follow-up questionnaire. To decrease the likelihood of attrition, participants were informed that they could win one of hundred cash prizes of $€ 100$ if they completed all questionnaires (Robroek et al., 2009). When participants did not fill out this questionnaire, they received a reminder after one and two weeks. If the questionnaire was still not completed after these reminders, respondents were called to administer a short telephone questionnaire.

\section{Intervention}

The web-based computer-tailored intervention was developed systematically using the Intervention Mapping protocol (Bartholomew et al., 2011). Detailed information about the development process and the content of the intervention can be found elsewhere (Walthouwer et al., 2013). The objective of the intervention was to prevent weight gain or achieve modest weight loss by making small changes in dietary intake and/or physical activity. In the video version, the core tailored information was provided by means of videos. These videos had a news-driven format in which professional actors read the tailored information aloud. This information was exactly the same as the information that could be read in text in the text version of the intervention. It should further be noted that the video version also consisted of text-driven information, particularly for delivering the general non-tailored information.

The theoretical framework of the intervention consisted of a combination of selfregulation theories (Leventhal et al., 1998; Zimmerman, 2000) and the I-Change Model (de Vries et al., 2003). The main determinants targeted in the intervention were knowledge, awareness, risk perception, attitude, social influence, self-efficacy, intention, skills, and action and coping planning. Mainly the following behavior change methods were used to bring about change in these determinants: consciousness raising, tailored feedback on behavior and cognitions, goal setting, action and coping planning, and evaluation of goal pursuit. The intervention consisted of six sessions and each session lasted about 15 minutes.

Session 1: The aim of session one was to inform participants about the different intervention sessions and to provide them with general information and tailored feedback about their weight, behavior (dietary intake and physical activity), and socio-cognitive beliefs towards improving their diet and physical activity level (risk perception, attitude, self-efficacy, and social influence). After receiving this information, participants subsequently had to set a goal by deciding if they wanted to maintain their current weight or lose a little weight. Participants also had to decide if they wanted to improve their physical activity level, their dietary intake, or both.

Session 2: The aim of session two was providing participants with detailed feedback on their chosen behavior in order to inform them which small changes they could 
make to achieve their weight goal. Based on this information, participants could make if then' plans by specifying when, where, and how they were going to undertake the behavior change. After session two, participants could start realizing their goals and plans.

Sessions 3-6: The last four sessions could be accessed in the subsequent weeks, with at least one week between the sessions. The aim of session three was to help participants carry out and maintain the behavior change by giving tailored feedback about their behavior change progress and offering them the possibility to receive tips about how to deal with hindering situations. Participants could make a coping plan for each chosen hindering situation by selecting a coping option from a list with pre-defined options. The last three sessions were identical to the third session but each new session also consisted of one or two new elements. Session four, for example, also consisted of narratives in which a role model told how his/her behavior change was going and how he/she dealt with difficult situations. Participants were further also given the possibility to change their goals and action plans. Session five was similar to session four, but in this session participants for the first time received tailored feedback on their weight change by indicating whether or not they had achieved their goal. Finally, session six was again similar to the previous session but additionally addressed the topic of how to maintain behavior changes on the long-term. For this purpose, participants had the possibility to set a long-term weight goal and make an action plan for achieving this goal. This last session ended with a recapitulation of the essential elements of the whole intervention, such as the steps to successfully self-regulate their behavior in future.

\section{Measurements}

\section{Outcome variables}

The outcome variables (i.e. BMI, dietary intake, and physical activity) were assessed using self-reports at both $\mathrm{T} 0$ and $\mathrm{T} 1$.

First, participants' body weight in kilograms and height in meters were assessed in order to calculate their BMI (weight/height ${ }^{2}$ ). To improve the adequacy of reporting, participants were asked to indicate their weight in the morning without clothes and shoes.

Dietary intake was assessed by means of a food frequency questionnaire consisting of 66 items, which was based on a validated questionnaire to assess fat intake (van Assema et al., 2001). The intake levels of mainly energy-dense products from six different food categories was assessed (i.e. dairy products, sandwiches and fillings, food at dinner, sweet and savory snacks, hot and cold beverages, and alcohol). For each food product, the frequency (i.e. number of days per week) and quantity (i.e. servings per day) were assessed. When applicable, type of product (e.g. use of skimmed, semi-skimmed, or whole milk) and portion size (e.g. size of candy bar) were assessed as well. These questions were combined with the energy value of each food product as indicated by the Dutch food composition table (RIVM, 2013) in order to calculate a score for the average daily intake of calories from energy-dense food products. 
Physical activity was assessed using the Short Questionnaire to Asses HealthEnhancing Physical Activity (SQUASH) (Wendel-Vos \& Schuit, 2002). This questionnaire has proven to be a reliable and valid tool to estimate the level of physical activity among Dutch adults (Wendel-Vos, Schuit, Saris, \& Kromhout, 2003). The SQUASH takes about five minutes to complete and assesses participants' level of physical activity per category (i.e. commuting activities, leisure time activities, household activities, and activities at work). For each activity, participants had to indicate on how many days per week they engage in this activity, average time per day spent in doing this activity, and the intensity of the activity (light, moderate, or vigorous). By combining the frequency, duration, and intensity of physical activity, a total score was calculated for the average daily minutes of moderate-to-vigorous intensity physical activity.

\section{Brief assessment of outcome measures}

Participants who had not completed the follow-up questionnaire after several email reminders were contacted by telephone to conduct a brief assessment of the outcome measures. The telephone questionnaire consisted of 11 items concerning participants' body weight, dietary intake, and physical activity level. The purpose of the telephone questionnaire was to integrate it with the online follow-up questionnaire in order to replace missing values on the outcome variables. Participants' body weight was assessed by asking about their current weight in the morning without clothes and shoes. Unfortunately, analyses showed that the measures of dietary intake and physical activity could not be compared with the measures obtained in the longer, validated online questionnaire.

\section{Demographics}

Demographic variables consisted of gender ( $1=$ male; $2=$ female), age, and educational level (i.e. the highest level of education completed) which was categorized into low (1=primary or basic vocational school), medium ( $2=$ secondary vocational school or high school), and high (3=higher vocational school or university) (UNESCO Institute for Statistics, 2012). These variables were all assessed at $\mathrm{T} 0$ and used to describe the study population as well as to correct for potential confounding variables in the effect analyses.

\section{Socio-cognitive variables}

All socio-cognitive variables (i.e. self-efficacy, intention, and self-regulation skills) were assessed at T0 using adapted measures of previous studies (Schulz, Kremers, et al., 2014; Stanczyk, Bolman, et al., 2014; van Genugten, van Empelen, Boon, et al., 2012) and a fivepoint Likert answering scale ranging from 1 (=low) to 5 (=high). These variables were assessed in order to correct for potential confounding variables in the effect analyses. A scale was computed by calculating a mean score. 
Participants' self-efficacy was measured separately for physical activity $(\alpha=.83)$ and dietary intake $(\alpha=.81)$ using four items per behavior. Participants were, for example, asked about their confidence and ability to improve their diet and physical activity level.

Intention was measured with one item per behavioral outcome by asking participants if they intended to improve their diet and physical activity level within the next six months.

Self-regulation skills were measured for the types of skills that are important for successfully translating intentions into behavior change (i.e. goal setting, action planning, monitoring, and coping planning). Items were derived from existing instruments (Brown, Miller, \& Lawendowski, 1999; Carey, Neal, \& Collins, 2004). Goal setting ( $\alpha=.72)$ was measured with three items by asking participants if they set a goal in advance when they, for example, want to manage their weight. Next, action planning was measured with three items per behavioral outcome. Participants were asked if they had a clear plan when, where, and how they wanted to improve their diet $(\alpha=.90)$ and their physical activity level $(\alpha=.94)$. Monitoring ( $\alpha=$.74) was measured using four general items. Participants were, for example, asked if they monitored their weight and behavior on a regular basis. Finally, two items per behavioral outcome were used to assess coping planning. Participants were asked to which degree they were able to identify hindering situations in advance and thought that they were able to deal with these situations for both diet $(\alpha=.70)$ and physical activity $(\alpha=.72)$.

\section{Process evaluation}

At T1, appreciation of the intervention was assessed by asking participants to which degree they thought the information and feedback in the intervention was interesting, useful, understandable, and fitted their own situation. Next, participants were asked about their feelings of autonomy, relatedness, and competence during the intervention. These concepts were derived from the Self-Determination Theory (Deci \& Ryan, 2010) and the items were developed using existing questionnaires (Soetens et al., 2014; Stanczyk, Bolman, et al., 2014; van Genugten, van Empelen, Boon, et al., 2012). Autonomy was assessed by two items. Participants were asked if they had the feeling that they could decide by themselves which goals they could set and which information they could read in the intervention. Relatedness was assessed with three items by asking participants if they felt involved and supported by the intervention. Competence was assessed with three items by asking participants if the intervention had increased their confidence in their ability to manage their weight, dietary intake, and physical activity behavior. Furthermore, participants were asked to give an overall rating of the intervention on a scale ranging from 1 (=low) to 10 (=high). All other questions could be answered on a five-point Likert scale, ranging from 1 (=low) to 5 (=high). Finally, login-data was used to determine how many participants actually used the intervention. 


\section{Statistical analyses}

At both T0 and T1, multiple imputation was used to replace missing values on the sociocognitive and outcome variables. Descriptive statistics and frequencies were used to describe the characteristics of the study population and the overall flow through the study. Baseline differences between the three study conditions were examined using analyses of variance with Tukey post-hoc tests for continuous variables and chi-square tests with Bonferroni correction $(P=0.05 / 3=.017)$ for categorical variables. To examine the possible presence of selective attrition between baseline and follow-up, a logistic regression analysis was performed with attrition at follow-up as outcome (not dropped out=0, dropped out=1) and study condition and all assessed baseline variables as predictors.

The effect analyses were conducted for each outcome variable separately (BMI, dietary intake, physical activity) using linear regression analyses with the enter method. These analyses were primarily conducted to examine the effects of the intervention conditions in comparison to the control condition. Therefore the study condition variable was recoded into two dummies (i.e. video versus control and text versus control). The analyses were adjusted for potential confounders (i.e. baseline behavior, predictors of attrition, and baseline differences). First, the analyses were run including study condition*educational level interaction terms in order to assess potential educational differences in intervention effects. When the interaction terms were significant, stratified analyses would be conducted. When interaction terms were not significant, the regression analyses were repeated without the interaction terms. Cohen's D effect sizes were calculated for all outcome variables (Cohen, 2013). As secondary analyses, we also compared the effects of the intervention conditions with each other. Moreover, complete case effect analyses were also performed in order to examine possible differences in results with the multiple imputation effect analyses.

Finally, the process evaluation data were analyzed. First, linear regression analyses with the enter method were performed including study condition*educational level interaction terms to identify potential educational differences in appreciation. When no interaction effects were found, independent samples t-tests were conducted to examine differences between the video and text condition on the process evaluation variables (i.e. appreciation).

All statistical analyses were conducted using SPSS 20.0, applying a significance level of 0.05 for single variables and 0.10 for interaction terms (Rosnow \& Rosenthal, 1989).

\section{Results}

\section{Study sample, baseline differences, and attrition analysis}

The CONSORT-EHEALTH flowchart (Eysenbach, 2011) (see Figure 1) shows the number of participants who were randomly assigned to one of the three study conditions as well as their flow through the study. In total, 1,419 participants completed the baseline 
questionnaire, of which 465 were assigned to the video condition, 491 to the text condition, and 463 to the control condition. At 6 month follow-up, data from 1,015 (71.5\%) participants was collected using the online and telephone questionnaires.

Figure 1. Flowchart of the enrollment, allocation, and participation of respondents

\begin{tabular}{|c|c|c|c|}
\hline$\stackrel{\Xi}{\Xi}$ & \multirow[b]{2}{*}{$\downarrow$} & $\begin{array}{l}\text { Completion of baseline questionnaire } \\
\qquad \mathrm{N}=1,419\end{array}$ & \\
\hline & & $\downarrow$ & $\downarrow$ \\
\hline 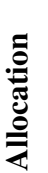 & $\begin{array}{l}\text { Video condition } \\
(\mathrm{N}=465) \\
\text { - Used intervention }(\mathrm{N}=328) \\
\text { - Did not use intervention } \\
(\mathrm{N}=137)\end{array}$ & $\begin{array}{l}\text { Text condition } \\
(\mathrm{N}=491) \\
\text { - Used intervention }(\mathrm{N}=364) \\
\text { - Did not use intervention } \\
(\mathrm{N}=127)\end{array}$ & Control condition $(\mathrm{N}=463)$ \\
\hline & $\downarrow$ & $\downarrow$ & $\downarrow$ \\
\hline 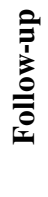 & $\begin{array}{l}\text { Completed follow-up } \\
\text { questionnaire }(\mathrm{N}=331) \\
\text { - Online }(\mathrm{N}=205) \\
\text { - Telephone }(\mathrm{N}=126) \\
\text { Lost to follow-up }(\mathrm{N}=134)\end{array}$ & $\begin{array}{l}\text { Completed follow-up } \\
\text { questionnaire }(\mathrm{N}=315) \\
\text { - Online }(\mathrm{N}=198) \\
\text { - Telephone }(\mathrm{N}=117) \\
\text { Lost to follow-up }(\mathrm{N}=176)\end{array}$ & $\begin{array}{l}\text { Completed follow-up } \\
\text { questionnaire }(\mathrm{N}=369) \\
\text { - Online }(\mathrm{N}=268) \\
\text { - Telephone }(\mathrm{N}=101) \\
\text { Lost to follow-up }(\mathrm{N}=94)\end{array}$ \\
\hline & $\downarrow$ & $\downarrow$ & $\downarrow$ \\
\hline 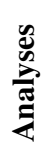 & $\begin{array}{l}\text { Analyzed multiple imputation } \\
(\mathrm{N}=465) \\
\text { Analyzed complete cases } \\
(\mathrm{N}=331)\end{array}$ & $\begin{array}{l}\text { Analyzed multiple imputation } \\
(\mathrm{N}=491) \\
\text { Analyzed complete cases } \\
(\mathrm{N}=315)\end{array}$ & $\begin{array}{l}\text { Analyzed multiple imputation } \\
(\mathrm{N}=463) \\
\text { Analyzed complete cases } \\
(\mathrm{N}=369)\end{array}$ \\
\hline
\end{tabular}

Table 1 provides a comprehensive overview of all baseline characteristics of the study sample, including baseline differences between the three study conditions. Participants' mean age was $48.1(\mathrm{SE}=0.31)$ and $58.6 \%$ were female. The mean BMI was $26.4(\mathrm{SE}=0.06)$ and $73.5 \%$ of the study sample was overweight. The majority had a high level of education (54.2\%), while fewest participants had a low educational level $(15.1 \%)$. The distribution of educational level differed significantly between the three study conditions (Pearson $\chi^{2}=10.38, P=.004$ ). Compared to the text and video condition, significantly more participants in the control condition had a low educational level. Moreover, the number of participants with a medium level of education was significantly higher in the text and control condition in comparison to the video condition. Lastly, participants' mean score on goal setting in the video condition was significantly higher in comparison to the text and control condition $(\mathrm{F}=4.740, P=.009)$. No other baseline differences were observed. 
Attrition analysis identified several significant predictors of drop-out. Participants in the video $(\mathrm{OR}=2.11,95 \% \mathrm{CI}=1.48-3.00, P=.000)$ and text condition $(\mathrm{OR}=3.23,95 \%$ $\mathrm{CI}=2.29-4.54, P=.000$ ) were significantly more likely to drop out compared to participants in the control condition. Attrition was further significantly higher among participants with a low $(\mathrm{OR}=2.15,95 \% \mathrm{CI}=1.46-3.16, P=.000)$ and medium $(\mathrm{OR}=1.37,95 \% \mathrm{CI}=1.02-1.85$, $P=.037)$ educational level in comparison to highly educated participants. Older participants were more likely to complete the follow-up questionnaire $(\mathrm{OR}=0.97,95 \% \mathrm{CI}=0.96-0.98$, $P=.000)$. Finally, participants who had lower levels of: self-efficacy to improve their diet $(\mathrm{OR}=1.36,95 \% \mathrm{CI}=1.06-1.76, P=.016)$, intention to improve their diet $(\mathrm{OR}=1.22,95 \%$ $\mathrm{CI}=1.02-1.46, P=.031)$, and coping planning regarding physical activity $(\mathrm{OR}=1.31,95 \%$ $\mathrm{CI}=1.04-1.64, P=.022)$ were significantly more likely to drop out.

Table 1. Characteristics of the study sample and differences between the study conditions

\begin{tabular}{|c|c|c|c|c|c|c|}
\hline Baseline characteristics & $\begin{array}{l}\text { Overall } \\
\text { sample } \\
(\mathrm{N}=1, \mathbf{4 1 9})\end{array}$ & $\begin{array}{l}\text { Video } \\
(\mathrm{N}=465)\end{array}$ & $\begin{array}{l}\text { Text } \\
(\mathrm{N}=491)\end{array}$ & $\begin{array}{l}\text { Control } \\
(\mathrm{N}=463)\end{array}$ & $\begin{array}{l}\text { F/Pearson } \\
\chi^{2}\end{array}$ & $P$ \\
\hline Gender (female), N (\%) & $831(58.6)$ & $273(58.7)$ & $284(57.8)$ & $274(59.2)$ & 0.18 & .913 \\
\hline Educational level, N (\%) & & & & & 10.38 & $.004 * *$ \\
\hline Low & $214(15.1)$ & $75(16.1)$ & $67(13.6)^{\mathrm{a}}$ & $72(15.6)^{\mathrm{a}}$ & & \\
\hline Medium & $436(30.7)$ & $118(25.4)^{\mathrm{a}, \mathrm{b}}$ & $161(32.8)^{\mathrm{a}}$ & $157(33.9)^{\mathrm{b}}$ & & \\
\hline High & $769(54.2)$ & $272(58.5)^{\mathrm{a}}$ & $263(53.6)$ & $234(50.5)^{\mathrm{a}}$ & & \\
\hline Age, mean (SE) & $48.1(0.31)$ & $48.1(0.09)$ & $47.8(0.08)$ & $48.5(0.08)$ & 0.41 & .667 \\
\hline $\begin{array}{l}\text { Self-efficacy improve physical } \\
\text { activity, mean (SE) }\end{array}$ & $3.3(0.02)$ & $3.4(0.01)$ & $3.4(0.01)$ & $3.3(0.01)$ & 0.56 & .571 \\
\hline $\begin{array}{l}\text { Self-efficacy improve diet, mean } \\
\text { (SE) }\end{array}$ & $3.3(0.02)$ & $3.3(0.00)$ & $3.3(0.00)$ & $3.2(0.00)$ & 0.83 & .436 \\
\hline $\begin{array}{l}\text { Intention improve physical } \\
\text { activity, mean (SE) }\end{array}$ & $4.0(0.03)$ & $4.0(0.01)$ & $4.0(0.01)$ & $4.0(0.01)$ & 0.05 & .953 \\
\hline $\begin{array}{l}\text { Intention improve diet, mean } \\
\text { (SE) }\end{array}$ & $4.1(0.03)$ & $4.1(0.01)$ & $4.1(0.01)$ & $4.0(0.01)$ & 0.65 & .520 \\
\hline Goal setting, mean (SE) & $3.5(0.02)$ & $3.6(0.01)^{\mathrm{a}, \mathrm{b}}$ & $3.5(0.01)^{\mathrm{a}}$ & $3.5(0.01)^{b}$ & 4.74 & $.009 * *$ \\
\hline $\begin{array}{l}\text { Action planning improve } \\
\text { physical activity, mean (SE) }\end{array}$ & $3.4(0.02)$ & $3.3(0.01)$ & $3.3(0.01)$ & $3.4(0.01)$ & 0.21 & .808 \\
\hline $\begin{array}{l}\text { Action planning improve diet, } \\
\text { mean (SE) }\end{array}$ & $3.2(0.02)$ & $3.2(0.01)$ & $3.2(0.01)$ & $3.3(0.01)$ & 0.69 & .502 \\
\hline Monitoring, mean (SE) & $3.3(0.02)$ & $3.3(0.01)$ & $3.3(0.01)$ & $3.4(0.01)$ & 0.66 & .520 \\
\hline $\begin{array}{l}\text { Coping planning improve } \\
\text { physical activity, mean (SE) }\end{array}$ & $3.4(0.02)$ & $3.4(0.00)$ & $3.4(0.01)$ & $3.4(0.01)$ & 0.75 & .475 \\
\hline $\begin{array}{l}\text { Coping planning improve diet, } \\
\text { mean (SE) }\end{array}$ & $3.3(0.02)$ & $3.3(0.01)$ & $3.3(0.01)$ & $3.3(0.01)$ & 0.06 & .941 \\
\hline BMI, mean (SE) & $26.4(0.06)$ & $26.4(0.02)$ & $26.5(0.02)$ & $26.4(0.02)$ & 0.13 & .878 \\
\hline $\begin{array}{l}\text { Average daily energy-intake, } \\
\text { mean (SE) }\end{array}$ & $1,296.9(13.40)$ & $1,308.4(3.56)$ & $1,314.7(3.51)$ & $1,266.5(3.75)$ & 1.33 & .266 \\
\hline $\begin{array}{l}\text { Average daily minutes moderate } \\
\text { and vigorous physical activity, } \\
\text { mean (SE) }\end{array}$ & $78.2(2.21)$ & $74.4(0.53)$ & $76.8(0.57)$ & $83.5(0.69)$ & 1.48 & .228 \\
\hline
\end{tabular}




\begin{tabular}{|c|c|c|c|c|}
\hline Follow-up characteristics & $\begin{array}{l}\text { Overall } \\
\text { sample } \\
(\mathbf{N}=1,419)\end{array}$ & $\begin{array}{l}\text { Video } \\
(\mathrm{N}=\mathbf{4 6 5})\end{array}$ & $\begin{array}{l}\text { Text } \\
(\mathrm{N}=491)\end{array}$ & $\begin{array}{l}\text { Control } \\
(\mathrm{N}=463)\end{array}$ \\
\hline BMI, mean (SE) & $26.1(0.08)$ & $25.9(0.02)$ & $26.1(0.02)$ & $26.2(0.02)$ \\
\hline $\begin{array}{l}\text { Average daily energy-intake, } \\
\text { mean (SE) }\end{array}$ & $1,072.6(20.79)$ & $1,016.5(3.56)$ & $1032.8(3.60)$ & $1,170.7(3.56)$ \\
\hline $\begin{array}{l}\text { Average daily minutes moderate } \\
\text { and vigorous physical activity, } \\
\text { mean (SE) }\end{array}$ & $107.7(5.71)$ & $103.2(0.80)$ & $108.4(0.80)$ & $111.8(0.88)$ \\
\hline \multicolumn{5}{|l|}{$* * P<0.01$} \\
\hline \multicolumn{5}{|c|}{$\begin{array}{l}* P<0.05 \\
\text { a, b } \text { Values within a row with identical letters were significantly different as determined by analyses of variance } \\
\text { with Tukey post-hoc test (for continuous variables) or chi-square tests with Bonferroni correction (for categorical }\end{array}$} \\
\hline
\end{tabular}

\section{Intervention effects on BMI, dietary intake, and physical activity}

We first examined the possible presence of interaction between type of study condition and educational level. There were no significant interaction effects for any of the outcome measures. Therefore, stratified analyses by educational level were not warranted.

The regression analyses without interaction terms showed several main intervention effects (see Table 2). We found that the video intervention resulted in a significantly lower BMI compared to the control condition $(\mathrm{B}=-0.25, P=.049)$, with a small Cohen's D effect size of .10 (Cohen, 2013). No significant difference was found between the text and control condition regarding BMI $(B=-0.09, P=.474)$. The analyses on dietary intake showed that both the video $(B=-175.58, P=.000)$ and text intervention $(B=-163.05$, $P=.001)$ resulted in a significantly lower average daily intake of calories from energy-dense food products compared to the control condition, with medium Cohen's D effect sizes of respectively .40 and .36 (Cohen, 2013). For physical activity no intervention effects were found for both the video $(\mathrm{B}=-1.45, P=.900)$ and text condition $(\mathrm{B}=1.88, P=.863)$ in comparison to the control condition.

In the additional analyses comparing the two intervention conditions, no significant differences were found for any of the outcome measures.

The complete case analyses resulted in the same significant findings as the effect analyses with the multiple imputation data that is described above.

\section{Process evaluation}

Table 3 provides an overview of the results from the process evaluation. In total, 355 participants completed the process evaluation questions. Overall, the mean scores of the process evaluation variables represented neutral to slightly positive scores for both versions of the intervention, without remarkable low scores. The intervention scored best on usefulness, understandability, and autonomy. The mean score for assessment of the intervention as a whole was 6.9 (SD 1.1) on the scale of 0 (=low) to 10 (=high). 
Regression analyses showed that there was no significant interaction effect of educational level regarding the process evaluation variables and, therefore, no stratified analyses were conducted. Independent samples t-tests showed that the information in the video condition was rated as more useful compared to the information provided in the text condition ( $\mathrm{t}=1.99, P=.047)$. Feelings of relatedness were also significantly higher among participants in the video condition $(\mathrm{t}=2.06, P=.041)$ as compared to the text condition. Finally, participants in the video condition rated the intervention significantly better than participants in the text condition $(\mathrm{t}=2.39, P=.018)$.

Table 2. Intervention effects on the outcome variables at follow-up as assessed by linear regression analyses

\begin{tabular}{|c|c|c|c|c|c|c|c|c|c|c|}
\hline \multirow{2}{*}{$\begin{array}{l}\text { Outcome } \\
\text { variables }\end{array}$} & \multicolumn{5}{|c|}{ Video $(1)$ versus control $(0)(n=928)^{a}$} & \multicolumn{5}{|c|}{ Text (1) versus control $(0)(n=954)^{a}$} \\
\hline & $\mathbf{B}$ & SE & $P$ & $95 \% \mathrm{CI}$ & ES & B & SE & $P$ & $95 \% \mathrm{CI}$ & ES \\
\hline BMI & -0.25 & 0.13 & $.049^{*}$ & $-0.50-0.00$ & .10 & -0.09 & 0.13 & .474 & $-0.35-0.16$ & .03 \\
\hline $\begin{array}{l}\text { Average daily } \\
\text { energy-intake }\end{array}$ & -175.58 & 45.13 & $.000 * *$ & $-265.24--85.92$ & .40 & -163.05 & 48.57 & $.001 *$ & $-259.78--66.32$ & .36 \\
\hline $\begin{array}{l}\text { Average daily } \\
\text { minutes moderate } \\
\text { and vigorous } \\
\text { physical activity }\end{array}$ & -1.45 & 11.48 & .900 & $-24.28-21.38$ & .01 & 1.88 & 10.88 & .863 & $-19.75-23.50$ & .02 \\
\hline
\end{tabular}

*** $P<0.01$

$* P<0.05$

${ }^{a}$ In the analyses the following covariates were included: baseline behavior, educational level, age, goal setting, self-efficacy to improve diet, coping planning regarding physical activity, and intention to improve diet.

$\mathrm{B}=$ Unstandardized regression coefficient; ES=Cohen's D effect size

Table 3. Mean and standard deviation (SD) of process evaluation variables at follow-up, including differences between the video and text condition

\begin{tabular}{|c|c|c|c|c|c|}
\hline Process evaluation variables & $\begin{array}{l}\text { Complete cases } \\
(\mathbf{N}=355)\end{array}$ & $\begin{array}{c}\text { Video } \\
(\mathbf{N}=177)\end{array}$ & $\begin{array}{c}\text { Text } \\
(\mathrm{N}=178)\end{array}$ & $\mathbf{t}$ & $P$ \\
\hline The feedback messages fit to my own situation & $3.4(0.9)$ & $3.4(0.9)$ & $3.3(0.9)$ & 0.87 & .387 \\
\hline The feedback messages were understandable & $3.9(0.8)$ & $3.9(0.8)$ & $3.8(0.8)$ & 0.77 & .441 \\
\hline The feedback messages were useful & $3.5(0.9)$ & $3.6(0.9)^{\mathrm{a}}$ & $3.4(0.9)^{\mathrm{a}}$ & 1.99 & $.047 *$ \\
\hline The feedback messages were interesting & $3.4(1.0)$ & $3.5(1.0)$ & $3.3(1.0)$ & 1.62 & .107 \\
\hline Feelings of autonomy & $4.0(0.8)$ & $4.1(0.7)$ & $3.9(0.8)$ & 1.82 & .070 \\
\hline Feelings of relatedness & $3.0(1.0)$ & $3.2(1.0)^{\mathrm{a}}$ & $2.9(1.0)^{\mathrm{a}}$ & 2.06 & $.041 *$ \\
\hline Feelings of competence & $3.2(1.0)$ & $3.2(1.0)$ & $3.1(1.0)$ & 1.50 & .135 \\
\hline Overall grade intervention (from 1 to 10 ) & $6.9(1.1)$ & $7.0(1.2)^{\mathrm{a}}$ & $6.7(1.1)^{\mathrm{a}}$ & 2.39 & $.018 *$ \\
\hline \multicolumn{6}{|l|}{$* * P<0.01$} \\
\hline$* P<0.05$ & & & & & \\
\hline
\end{tabular}




\section{Discussion}

\section{Principal findings}

The aim of this study was to evaluate the effects and appreciation of a video and text version of a web-based computer-tailored obesity prevention intervention among Dutch adults with a high and low level of education.

Our results show that there were no significant group*education interaction effects, implying that no stratified analyses were warranted and that both versions of the intervention were equally effective for all educational levels. The video version was the most effective intervention since it had a positive significant effect on self-reported BMI and dietary intake (compared to the control condition), while the text version only had a significant positive effect on dietary intake. No intervention effects on physical activity were found. Appreciation of the two intervention versions did also not differ per educational level. Yet, the video version was appreciated better than the text version on several aspects of importance for intervention use (i.e. usefulness of messages, feelings of relatedness, and grade given to intervention). Overall, it can be concluded that the video intervention performed better than the text intervention regardless of people's educational level.

Although our findings show that the video version was the most effective intervention for both lower and higher educated participants, this also implies that we did not find support for our hypothesis (i.e. video version more effective for people with a low educational level). This finding, however, is in line with a recent study that has used the same study design to test the effects of a video web-based computer-tailored smoking cessation intervention. In this study, a main effect of the video condition was demonstrated as well but differential effects per educational level could not be found either (Stanczyk, Bolman, et al., 2014). Furthermore, our hypothesis that video messages may work better for lower educated people (Walthouwer et al., 2013) was based on indications and assumptions derived from few previous studies but the evidence for this hypothesis was not compelling. In conclusion, our study adds to the evidence that the use of videos in a web-based computer-tailored intervention is effective in changing health behaviors regardless of educational level (Stanczyk, Bolman, et al., 2014).

Both the video and text version had the strongest effect on dietary intake, a finding in line with two reviews on web-based computer-tailored interventions (Kroeze et al., 2006; Neville et al., 2009a). The medium effect size indicates that this effect also is of clinical relevance (Cohen, 2013) and may suggest an important public health potential when the intervention is implemented at a large scale. In line with other studies (Broekhuizen et al., 2012; Wieland et al., 2012), only a small effect size was found regarding BMI (of the video version). This can be explained by the fact that BMI is a distal outcome and effects on BMI are generated by changes in energy intake and energy expenditure. However, even small intervention effects on BMI can have a large public health impact resulting in a significant reduction of many health problems, an improved quality of life, and cost savings 
(Finkelstein et al., 2012; Hill, 2009; Stevens et al., 2001; Verkleij, Adriaanse, Wendel-Vos, $\&$ Schuit, 2012). The fact that the text version did not have a significant effect on BMI can possibly be explained by the fact that the Cohen's D effect size for dietary intake (.36) was somewhat smaller compared to the effect size of the video version (.40). The fact that no intervention effect was found on physical activity is not surprising. Many reviews have reported mixed findings of web-based computer-tailored interventions for in particular physical activity with some studies reporting positive findings and others negative findings (Enwald \& Huotari, 2010; Kroeze et al., 2006; Neville et al., 2009b). One explanation for this can be that we encountered problems with the measurement of physical activity in our study. As can be seen in Table 1, the average daily minutes of moderate- to vigorousintensity physical activity scores were unrealistically high and about three quarters of our sample met the Dutch physical activity standard. Consequently, many participants did not receive the advice to increase their physical activity level within the intervention resulting in little improvement for this behavior. Recently, this problem has also been identified in a similar efficacy study and future studies should therefore take this problem into account when assessing physical activity and developing algorithms to deliver tailored messages (Schulz, Kremers, et al., 2014).

In line with two recent studies (Soetens et al., 2014; Stanczyk, Bolman, et al., 2014), it can further be concluded that a web-based computer-tailored intervention consisting of videos is appreciated better than an identical intervention that merely consists of text. This difference in appreciation can possibly also explain why the video version was more effective than the text version. The Elaboration Likelihood Model (Petty \& Cacioppo, 1986b), for example, suggests that when information is perceived as interesting and attractive it is more likely that central route processing will occur. Information that is processed via this central path will have a more long lasting persuasive effect on the receiver (Petty \& Cacioppo, 1986a). The better appreciation of the video version may therefore have resulted in more central route information processing. It further also is important that interventions are appreciated well because interventions that are appreciated better are more likely to be used and this will in turn increase their effectiveness (Magnée et al., 2013). This is in particular important for interventions that consist of multiple sessions, such as our intervention. Hence, to facilitate appreciation and accordingly the use and effectiveness of future web-based computer-tailored interventions, we recommend using videos as delivery format. However, since it is relatively expensive and time consuming to develop video messages, first more research is needed about the cost-effectiveness of both the video and text version. Based on the high drop-out rate, future research should further also aim to study how the use of the intervention could be improved. The video version can, for example, possibly be improved by demonstrating how self-regulation skills can be applied in practice instead of only reading the tailored message aloud. 


\section{Strengths and limitations}

An important strength is that, to our knowledge, this is the first study that has studied how the effectiveness and attractiveness of a web-based computer-tailored obesity prevention intervention can be improved among people with a low educational level. Thus far, only few studies have addressed the issue of improving the impact of these interventions among lower educated people (Magnée et al., 2013). Another strength is that our intervention met several criteria related to higher effectiveness of weight management interventions, such as the self-regulation (Leventhal et al., 1998; Magnée et al., 2013; Zimmerman, 2000) and small changes approach (Hill, 2009) as well as the use of the Intervention Mapping protocol to develop an intervention fully grounded on theory and empirical evidence (Bartholomew et al., 2011). A final strength is that we found exactly the same results regardless if the analyses were carried out with the multiple imputation data or the complete cases data. This provides further support for our findings.

A limitation of this study is that all outcome measures were self-reported (Matthews \& Welk, 2002; Schwarz, 1999). We tried to gather objective data for BMI by working together with occupational health centers, but this recruitment strategy was unfortunately unsuccessful. However, research has shown that self-reported BMI does not affect results when used as continuous variable in a longitudinal study (Dahl, Hassing, Fransson, \& Pedersen, 2010). As mentioned before, participants' physical activity scores were unrealistically high and future studies should therefore aim to use more reliable online measures for physical activity. Moreover, although our drop-out rate is somewhat lower compared to previous research (Bennett \& Glasgow, 2009; Evers et al., 2005; Eysenbach, 2005; Soetens et al., 2014), it still is relatively high and implies that the attractiveness of the intervention deserves further attention. The high drop-out also caused many missing values on the outcome variables at follow-up, which, however, were replaced adequately using multiple imputation. Possibly, when drop-out rates would have been lower and intervention use higher, the effect sizes would have been higher (Magnée et al., 2013). Finally, we used a relatively short follow-up period of 6 months. Research with a longer follow-up period is necessary to examine whether or not the intervention effects will be maintained on the long-term.

\section{Conclusions}

Based on our results, it can be concluded that a web-based computer-tailored obesity prevention intervention is most effective and attractive when it consists of video messages. Hence, the impact of future web-based computer-tailored obesity prevention interventions can be improved by including videos as delivery format of tailored health information. Our study shows that this is feasible and effective for Dutch adults with a healthy weight and limited overweight. However, more research with a longer follow-up period is needed to study if it is cost-effective to use videos as delivery format and how in particular people with a low educational level can be reached more effectively. 


\section{CHAPTER 6}

Use and effectiveness of a video and text version of a webbased computer-tailored obesity prevention intervention: does delivery format matter?

Accepted as: Walthouwer, M., Oenema, A., Lechner, L., \& de Vries, H. Use and effectiveness of a video- and text-driven web-based computer-tailored intervention: does delivery format matter? Journal of Medical Internet Research. 


\begin{abstract}
Background: Many web-based computer-tailored interventions are characterized by high drop-out rates. This limits the potential impact of these interventions.

Objective: This study had three aims: (1) examining if the use of a web-based computertailored obesity prevention intervention can be increased by using videos as delivery format and whether more use was related to better intervention effects; (2) examining if the delivery of intervention content via participants' preferred delivery format can increase intervention use and whether this is related to better intervention effects; (3) identifying which socio-demographic factors and intervention appreciation variables predict intervention use.

Methods: Data was used of a randomized controlled study into the efficacy of a video and text version of a web-based computer-tailored obesity prevention intervention, consisting of a baseline measurement, 6 weekly intervention sessions, and a 6-month follow-up measurement. ANCOVAs were conducted to assess differences in use between the video and text version and between participants allocated to a matching and mismatching intervention delivery format. Potential moderation by intervention use and (mis)matching delivery format on self-reported BMI, physical activity, and energy-intake was examined using regression analyses with interaction terms. Finally, regression analysis was performed to assess determinants of intervention use.
\end{abstract}

Results: In total 1,419 participants completed the baseline questionnaire (follow-up response $=71.5 \%$ ). Intervention use declined rapidly over time: the first two intervention sessions were completed by approximately half of the participants and only $10.9 \%$ of the study population completed all six sessions of the intervention. There were no significant differences in use between the video and text version. Intervention use was significantly higher among participants who were allocated to an intervention condition that matched their preferred intervention delivery format. There were no significant interaction terms for any of the outcome variables: a match and more intervention use were not associated with better intervention effects. Participants with a high BMI and participants who felt involved and supported by the intervention were more likely to use the intervention more often.

Conclusions: The use of web-based computer-tailored interventions can be improved by delivering intervention content via participants' preferred intervention delivery format and creating feelings of relatedness. Yet, first more research into this is needed because a match and more intervention use had not resulted in better intervention outcomes.

Keywords: intervention use, web-based, computer-tailoring, obesity, educational level, delivery strategy, matching 


\section{Introduction}

Web-based computer-tailored interventions are increasingly being tested to target healthrelated behaviors, such as physical activity, dietary intake, and smoking (Brug et al., 2003; Tate, Finkelstein, Khavjou, \& Gustafson, 2009). These interventions are potentially costeffective since they can reach many people with individualized information via the Internet for relatively low costs (Brug et al., 2003; Schulz, Smit, et al., 2014; Smit, Evers, et al., 2013; Wieland et al., 2012). Unfortunately, these interventions are often not optimally used by the intended target groups. A rapid decline in use of intervention sessions in the first weeks after initial participation is often seen, in particular among people with a low educational level (Bennett \& Glasgow, 2009; Glasgow, 2007; Leslie, Marshall, Owen, \& Bauman, 2005; Magnée et al., 2013; Schulz et al., 2012; Spittaels, de Bourdeaudhuij, \& Vandelanotte, 2007; Wangberg et al., 2008). As a result many people will not be exposed to all essential intervention content, that is often provided in multiple sessions that can be worked through over a longer period of time. This limits the potential impact of such interventions as evidence suggests that repeated intervention use is necessary to achieve sustainable behavioral changes (Eysenbach, 2005; Hansen et al., 2012; Lenert et al., 2003; Strecher et al., 2008; Vandelanotte et al., 2007; Verheijden, Jans, Hildebrandt, \& HopmanRock, 2007; Wantland, Portillo, Holzemer, Slaughter, \& McGhee, 2004). Hence, many scholars have highlighted the necessity to increase research on strategies that can improve the (prolonged) use and correspondingly the efficacy of web-based computer-tailored interventions, particularly among the highly relevant target group of people with a low educational level (Brouwer et al., 2011; Magnée et al., 2013; Schneider et al., 2012; Schulz et al., 2012; Stanczyk, Crutzen, Bolman, Muris, \& de Vries, 2013). However, although research into this area is growing, thus far studies yielded no or only modest improvements in intervention use (Bennett \& Glasgow, 2009; Schulz et al., 2012).

Hence, the main aim of the present study was to contribute to the required insight into how the use of web-based computer-tailored interventions can be improved. This study is part of a randomized controlled trial (RCT) that has demonstrated that a video version of a web-based computer-tailored obesity prevention intervention is more effective in reducing body mass index (BMI) and energy-intake than a similar text version (Walthouwer, Oenema, Lechner, \& de Vries, submitted). In line with this finding, the first study aim was to examine if the video version had also resulted in more intervention use compared to the text version among people with a low educational level in particular and whether more use was related to better intervention effects. To provide additional insight into possible ways to increase intervention use, the second study aim was to examine the assumption of prior research that the delivery of intervention content via persons' preferred intervention delivery format can increase intervention use and is related to better intervention outcomes (Dijkstra, Conijn, \& de Vries, 2006; Kiesler \& Auerbach, 2006; King, 2000; Swift \& Callahan, 2009; Williams-Piehota, Schneider, Pizarro, Mowad, \& 
Salovey, 2003). In addition, the third study aim was examining demographic factors and intervention appreciation variables as potential predictors of intervention use.

The idea to examine the additional effects of videos has originated from the fact that most web-based computer-tailored interventions merely consist of 'dry' text-based information. Video-based messages are livelier and therefore more likely to be engaging and to stimulate revisits (Alley et al., 2014; Lee, 2011; Stanczyk et al., 2011; Vandelanotte \& Mummery, 2011; Walthouwer et al., 2013). The use of videos may in particular be appropriate for persons with a low educational level since these individuals generally are less text-oriented (Paasche-Orlow et al., 2005; Stanczyk et al., 2013; Walthouwer et al., 2013). In addition, previous studies in varying research fields have shown that matching the delivery format of intervention content with participants' preferred intervention delivery format facilitates prolonged intervention use, decreases drop-out, and eventually leads to better health outcomes (Dijkstra et al., 2006; Kiesler \& Auerbach, 2006; King, 2000; Swift \& Callahan, 2009; Williams-Piehota et al., 2003). The Elaboration Likelihood Model has further indicated that an adequate match between persons' preferences and educational materials will stimulate central route processing, which accordingly makes it more likely that positive changes are induced (Dijkstra, 2008). Conversely, a mismatch can reduce participation and may result in negative outcomes such as dissatisfaction with the information and eventually result in poorer intervention effects (Kiesler \& Auerbach, 2006; King, 2000).

The use of web-based computer-tailored interventions can also be improved by gaining more insight into the factors that are predictive for intervention use. Prior research has, for example, suggested that interventions that are appreciated well are more likely to be used (Brouwer et al., 2011; Brouwer et al., 2008, 2009; Peels, de Vries, et al., 2013). Based on the Self-Determination Theory (SDT), it has further been suggested that intervention use may be higher when an intervention is evaluated well on factors that can increase a person's intrinsic motivation, such as perceived autonomy and relatedness (Deci \& Ryan, 2011; Ryan, Patrick, Deci, \& Williams, 2008). Research has also demonstrated that intervention use is influenced by demographic factors: men and people with a low educational level are more likely to discontinue a web-based computer-tailored intervention, while intervention use is higher among women and older persons (Brouwer et al., 2010; Schulz et al., 2012; Verheijden et al., 2007). More insight into the demographic characteristics predictive for intervention use offers the possibility to improve future interventions by including promotional strategies to engage and encourage revisits among people who are less likely to revisit a web-based computer-tailored intervention (Schulz et al., 2012).

In conclusion, the main aim of this study was to provide insight into how the use and effectiveness of web-based computer-tailored interventions can be improved. 


\section{Methods}

The Ethical Committee of the Open University Heerlen has reviewed the study protocol and has decided that there was no objection to performance of the study. The study is registered in the Dutch Trial Register (NTR3501).

\section{Study design and respondents}

For this study, we used data from a RCT into the efficacy of the video and text version of the web-based computer-tailored obesity prevention intervention. These two versions were compared to a waiting list control group. Baseline measurements (T0) took place between September 2012 and February 2013 and there was one follow-up measurement six months after baseline (T1). Participants were eligible to participate when they were at least 18 years old, had a paid job, a BMI between 18.5 and 30, and sufficient command of the Dutch language. People who had a physical condition that influenced their dietary or physical activity pattern (e.g. diabetes) were excluded from participation.

Participants were recruited during health screenings performed by occupational health centers, but mainly directly through worksites and via advertisements in newspapers. Participants had to register at the study website, where they could read more information about the study and the intervention. After registration, participants were randomly assigned to one of the three study conditions (video version, text version, control group) in a computer determined sequence after which they received a username and password by email. With their account they could $\log$ into the website and fill out the baseline questionnaire. Participants assigned to one of the two intervention conditions were given access to the intervention two weeks after completion of the baseline questionnaire. To decrease the likelihood of attrition, participants received two email reminders per questionnaire. Participants could further win one of hundred cash prizes of $€ 100$,- if they completed all questionnaires (Robroek et al., 2009).

\section{Intervention}

The video and text version of the web-based computer-tailored intervention had the objective to prevent weight gain or achieve modest weight loss by guiding people in making and maintaining small changes in dietary intake and physical activity. Both versions had exactly the same content. Yet, the core tailored feedback in the video version was provided via videos, whereas the text version provided the intervention content merely via text without any visual elements. In the videos professional actors read the messages aloud by means of a news-driven format. The video version further also contained some textual information regarding, for example, the goal setting and action planning tools. The I-Change Model (de Vries et al., 2003) and self-regulation theories (Leventhal et al., 1998; Zimmerman, 2000) were used as the theoretical basis of the intervention. All educational content that was necessary to guide users through all the steps of the self-regulation process was delivered in six different intervention sessions, which each lasted about 15 minutes. To 
decrease the likelihood of attrition, participants received two email reminders per session. Detailed information about the development of the intervention can be found elsewhere (Walthouwer et al., 2013).

The aim of session one was to help participants set an appropriate weight goal (i.e. maintain current weight or lose a little weight) and a behavior change goal (i.e. improve dietary intake, physical activity, or both). To guide participants in making a choice, they received tailored feedback about their BMI, dietary intake, and physical activity level as well as feedback on their socio-cognitive beliefs toward making changes in diet and physical activity (i.e. attitude, self-efficacy, and social influence).

Session two aimed to help participants make appropriate 'if then' action plans. For this purpose, participants first received extensive feedback on the chosen behavior in which it was indicated which specific behavior changes they could make in order to achieve their weight goal. Subsequently, participants had to specify when, where, and how they were going to perform the desired behavior change. After this session, participants could start with the planned behavior change.

The last four sessions could be accessed in the next weeks, with at least one week between each session. The main aim of these sessions was to indicate whether or not participants had achieved their goals. Session three, for example, provided tailored feedback about participants' behavior change progress and offered the possibility to make coping plans. In addition, session four also consisted of narratives in which a role model told how his/her behavior change was going and how he/she dealt with difficult situations. In this session, participants also received the possibility to change their goals and plans. Session five was similar to session four, but also provided iterative feedback concerning participants' success in attaining their weight goal. Finally, session six was again similar to the previous session but also offered the possibility to set a long-term weight goal and make plans for achieving this goal.

\section{Measurements}

\section{Outcome variables}

Intervention use was assessed by examining how many sessions were completed during the whole intervention period. Based on website tracking data, it was possible to assess whether or not participants had completed a particular intervention session. For each session, completion was scored as 1 and non-completion as 0 . These scores were summed which resulted in a total score for intervention use ranging from 0 to 6 completed sessions.

Participants' dietary intake was assessed at both T0 and T1 by means of a food frequency questionnaire consisting of 66 items. This questionnaire was based on a validated questionnaire concerning fat intake (van Assema et al., 2001) and mainly assessed intake of energy-dense products originating from six different food categories (i.e. dairy products, sandwiches and fillings, food at dinner, sweet and savory snacks, hot and cold beverages, and alcohol). For each food product, the frequency (i.e. number of days per week) and 
quantity (i.e. servings per day) were assessed and when applicable portion size and type of product (e.g. use of skimmed, semi-skimmed, or whole milk) were assessed as well. A score for the average daily intake of calories from energy-dense food products was calculated by combining these questions with the energy value of each food product as indicated by the Dutch food composition table (RIVM, 2013).

At T0 and T1, physical activity was assessed using the Short Questionnaire to Asses Health-Enhancing Physical Activity (SQUASH) (Wendel-Vos et al., 2003; WendelVos \& Schuit, 2002). Research has shown that this is a reliable and valid questionnaire to estimate the level of physical activity among Dutch adults (Wendel-Vos et al., 2003; Wendel-Vos \& Schuit, 2002). Per category (i.e. commuting activities, leisure time activities, household activities, and activities at work), participants had to indicate on how many days per week they engage in this activity, the average time per day spent in doing this activity, and the intensity of the activity (i.e. light, moderate, or vigorous). The scores on these questions were used to calculate a total score for the average daily minutes of moderate and vigorous intensity physical activity.

To assess BMI (weight/height ${ }^{2}$ ), participants were asked to report their height in meters and their body weight in kilograms as measured in the morning without clothes and shoes at both $\mathrm{T} 0$ and $\mathrm{T} 1$. In addition, participants who had not completed the online followup questionnaire at T1 were contacted by telephone to assess their body weight. In line with the online questionnaire, these participants were asked to indicate their body weight in kilograms as measured in the morning without clothes and shoes.

\section{Intervention delivery format preference}

To assess participants' preferred intervention delivery format, they were asked at baseline to indicate via which delivery format they preferred to receive information in web-based computer-tailored interventions (text only, videos only, combination of text and videos, or no preference). The answer to this question was subsequently combined with the assigned study condition to determine whether or not participants' preference matched with the delivery format of the allocated intervention condition. This resulted in two groups of participants: (1) participants with a matched preference (i.e. participants allocated to text condition and delivery preference for text only and participants allocated to video condition and delivery preference for a combination of video and text) and (2) participants with a mismatched preference (i.e. participants allocated to text condition and delivery preference for video only or combination of video and text and participants allocated to video condition and delivery preference for text only or video only). It should be noted that participants who indicated that they did not have a preference for a particular intervention delivery format were not included in this variable $(\mathrm{N}=320)$. 


\section{Appreciation of intervention}

At T1, appreciation of the intervention was assessed by means of eight concepts. First, participants were asked to indicate on a five-point Likert scale (1=low and 5=high) how they appreciated the information and feedback messages in the intervention: interesting, useful, understandable, and fitting to own situation. In addition, participants were also asked to give an overall rating of their impression of the intervention on a scale ranging from 1 (=very poor) to 10 (=excellent). Finally, participants' perceptions of the intervention regarding autonomy, relatedness, and competence were assessed. These three concepts were derived from the SDT (Deci \& Ryan, 2011) and measured on a five-point Likert scale ( 1 =low and $5=$ high). First autonomy was assessed with two items by asking participants to which degree they experienced freedom in setting own goals and plans as well as in deciding which information they could read. Next, competence was assessed using three items. Participants had to indicate if the intervention had increased their confidence in their ability to manage their weight, dietary intake, and physical activity behavior. Finally, relatedness was assessed using three items by asking participants if they felt involved and supported by the intervention. Eventually, for each of the three SDT concepts a mean score was calculated.

\section{Demographics}

Demographic characteristics were assessed at T0 and included gender ( $1=$ male; $2=$ female), age, and educational level (i.e. the highest level of education completed). Educational level was classified into three categories: low (1=primary or basic vocational school), medium ( $2=$ secondary vocational school or high school), and high ( $3=$ higher vocational school or university) (UNESCO Institute for Statistics, 2012).

\section{Statistical analyses}

All statistical analyses were conducted using SPSS 20.0, applying a significance level of 0.05 for single variables and 0.10 for interaction terms (Rosnow \& Rosenthal, 1989).

Descriptive statistics and frequencies were used to describe the demographic characteristics of the study population at baseline as well as use of the different intervention sessions. Potential differences between the three study conditions at baseline were examined using analyses of variance (ANOVA) with Tukey post-hoc tests for continuous variables and chi-square tests with Bonferroni correction for categorical variables.

Difference in use between the video and text intervention was assessed using analysis of covariance (ANCOVA). Another ANCOVA was performed to examine differences in use between the video and text intervention per educational level. Difference in use between participants who were assigned to an intervention condition that matched or mismatched their preferred intervention delivery format was also assessed with an ANCOVA. 
To examine whether the intervention effects were moderated by (1) intervention use and (2) (mis)matching intervention delivery format, linear regression analyses with interaction terms (i.e. study condition*intervention use and study condition*(mis)matching intervention delivery format) were performed. Separate analyses were conducted for each outcome variable (i.e. BMI, dietary intake, physical activity) and the effects of the intervention conditions were examined in comparison to the control condition, for which the study condition variable was recoded into two dummies (i.e. video versus control and text versus control). The regression analyses were further adjusted for potential confounders (e.g. baseline behavior and baseline differences). In addition, all moderation analyses were performed with both a multiple imputation as completers only dataset.

Finally, a multiple linear regression analysis with the enter method was carried out to assess which demographics and intervention appreciation variables predicted intervention use.

\section{Results}

\section{Study sample}

The CONSORT-EHEALTH flowchart (Eysenbach, 2011) shows the number of participants who were randomly assigned to one of the three study conditions as well as their use of the intervention and participation throughout the study (see Figure 1). In total, 1,419 participants had completed the baseline questionnaire and at 6-month follow-up data was collected of 1,015 (71.5\%) participants. Of the participants who had completed the baseline questionnaire, only 328 (70.5\%) participants in the video condition and $364(74.1 \%)$ in the text condition also completed the first intervention session. Session one and two were completed by about half of the participants and only $44(9.5 \%)$ participants in the video condition and $60(12.2 \%)$ in the text condition followed all intervention sessions. Overall, only $10.9 \%$ of the participants completed all six sessions of the intervention.

Participants' mean age was $48.1(\mathrm{SD}=11.5)$ and 831 (58.6\%) participants were female (see Table 1). The distribution of educational level between the three study conditions differed significantly (Pearson $\chi^{2}=10.38, P=.004$ ) at baseline (see Table 1). The number of participants with a low educational level was significantly higher in the control condition compared to the text condition. Moreover, the number of participants with a medium educational level was significantly higher in the text and control condition in comparison to the video condition. In addition, compared to the control condition significantly more participants in the video condition had a high educational level.

Most participants preferred to receive information in web-based computer-tailored interventions via text only $(40.8 \%)$ followed by a combination of video and text $(34.5 \%)$. Only $30(2.1 \%)$ participants preferred to receive information via video only and 320 $(22.6 \%)$ participants had no preference regarding the delivery format. In total, $368(48.6 \%)$ participants were assigned to an intervention condition that matched their preferred intervention delivery format. For example, 206 (42.0\%) participants who were assigned to 
the text condition also preferred to receive information in web-based computer-tailored interventions via text only. The distribution of (mis)matching intervention delivery format between the two intervention conditions differed significantly (Pearson $\chi^{2}=4.74, P=.030$ ). Compared to participants in the control condition, significantly more participants in the text condition had an intervention delivery format preference for video only or a combination of video and text. This result was also found when we included participants who had no preference for a particular intervention delivery format in the matching group (Pearson $\left.\chi^{2}=8.31, P=.004\right)$.

Figure 1. Flowchart of the enrollment and participation of respondents

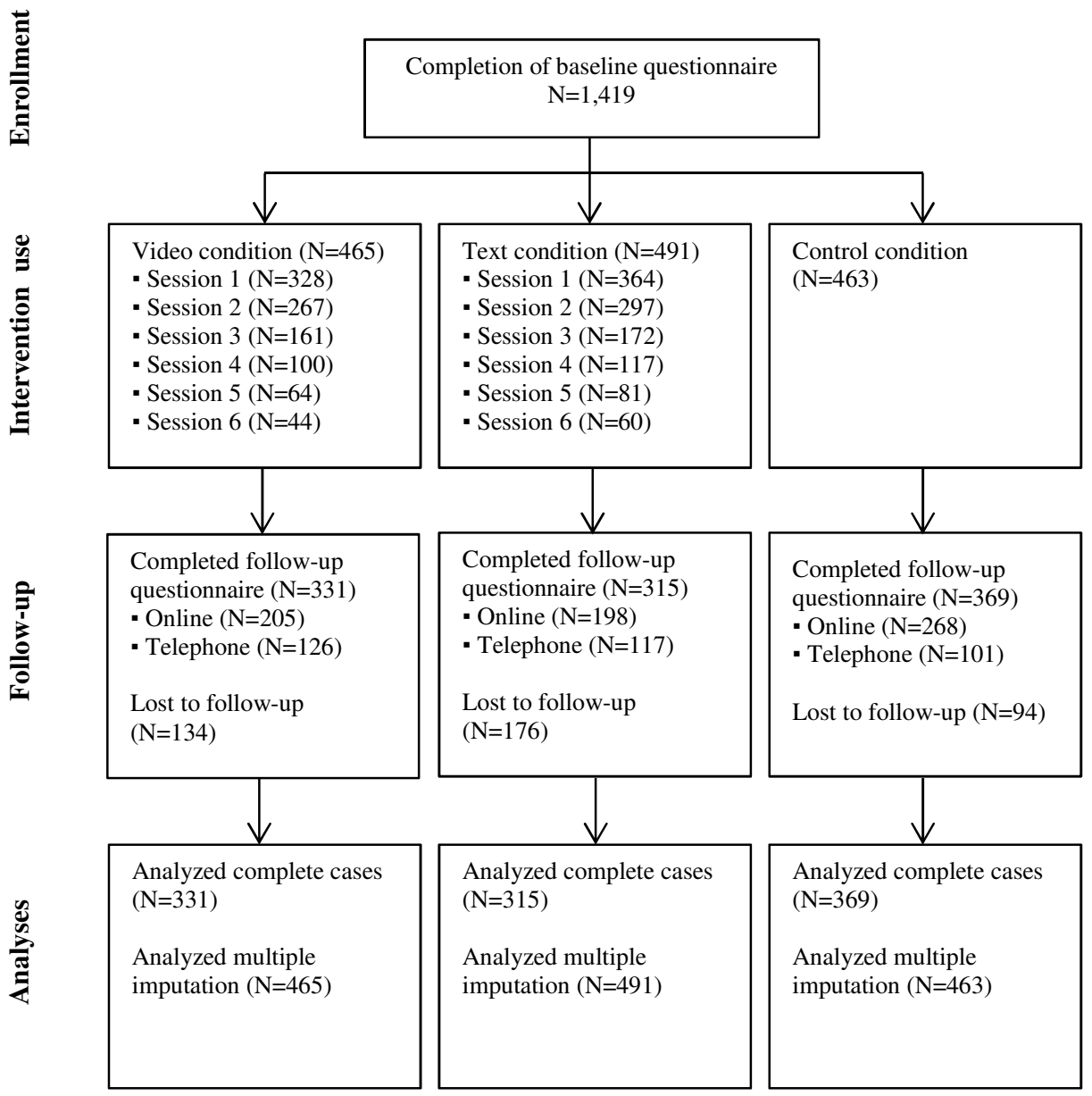


Table 1. Characteristics of the study sample and differences between the study conditions

\begin{tabular}{|c|c|c|c|c|c|c|}
\hline Baseline & $\begin{array}{l}\text { Full sample } \\
(\mathrm{N}=1,419)\end{array}$ & $\begin{array}{l}\text { Video } \\
(\mathrm{N}=465)\end{array}$ & $\begin{array}{l}\text { Text } \\
(\mathrm{N}=491)\end{array}$ & $\begin{array}{l}\text { Control } \\
(\mathrm{N}=463)\end{array}$ & $\begin{array}{l}\text { F/Pearson } \\
\chi^{2}\end{array}$ & $P$ \\
\hline Gender (female), n (\%) & $831(58.6)$ & $273(58.7)$ & $284(57.8)$ & $274(59.2)$ & 0.18 & .913 \\
\hline Educational level, n (\%) & & & & & 10.38 & $.004 * *$ \\
\hline Low & $214(15.1)$ & $75(16.1)$ & $67(13.6)^{\mathrm{a}}$ & $72(15.6)^{\mathrm{a}}$ & & \\
\hline Medium & $436(30.7)$ & $118(25.4)^{\mathrm{a}, \mathrm{b}}$ & $161(32.8)^{\mathrm{a}}$ & $157(33.9)^{b}$ & & \\
\hline High & $769(54.2)$ & $272(58.5)^{\mathrm{a}}$ & $263(53.6)$ & $234(50.5)^{\mathrm{a}}$ & & \\
\hline Age, mean (SD) & $48.1(11.5)$ & $48.1(12.1)$ & $47.8(11.6)$ & $48.5(10.9)$ & 0.41 & .667 \\
\hline BMI, mean (SD) & $26.4(2.3)$ & $26.4(2.3)$ & $26.5(2.4)$ & $26.4(2.4)$ & 0.13 & .878 \\
\hline $\begin{array}{l}\text { Average daily minutes } \\
\text { moderate and vigorous } \\
\text { physical activity, mean (SD) }\end{array}$ & $78.2(83.4)$ & $74.4(73.3)$ & $76.8(81.1)$ & $83.5(94.5)$ & 1.48 & .228 \\
\hline $\begin{array}{l}\text { Average daily energy-intake, } \\
\text { mean (SD) }\end{array}$ & $1,296.9(501.0)$ & $1,308.4(490.4)$ & $1,314.7(497.4)$ & $1,266.5(515.1)$ & 1.33 & .266 \\
\hline $\begin{array}{l}\text { Intervention delivery format } \\
\text { preference, } \mathrm{n}(\%)\end{array}$ & & & & & 6.76 & .344 \\
\hline Text only & $579(40.8)$ & $194(41.7)$ & $206(42.0)$ & $179(38.7)$ & & \\
\hline Video only & $30(2.1)$ & $8(1.7)$ & $12(2.4)$ & $10(2.2)$ & & \\
\hline Combination video/text & $489(34.5)$ & $162(34.8)$ & $175(35.7)$ & $152(32.8)$ & & \\
\hline No preference & $320(22.6)$ & $101(21.7)$ & $97(19.8)$ & $122(26.3)$ & & \\
\hline $\begin{array}{l}\text { Matching intervention } \\
\text { delivery format, } \mathrm{n}(\%)\end{array}$ & & & & & 4.74 & $.030 *$ \\
\hline Match & $368(48.6)$ & $162(44.5)^{\mathrm{a}}$ & $206(52.4)^{\mathrm{a}}$ & - & & \\
\hline Mismatch & $389(51.4)$ & $202(55.5)^{\mathrm{a}}$ & $187(47.6)^{\mathrm{a}}$ & - & & \\
\hline Follow-up & $\begin{array}{l}\text { Full sample } \\
(\mathrm{N}=\mathbf{1 , 0 1 5})\end{array}$ & $\begin{array}{l}\text { Video } \\
(\mathbf{N}=331)\end{array}$ & $\begin{array}{l}\text { Text } \\
(\mathrm{N}=315)\end{array}$ & $\begin{array}{l}\text { Control } \\
(\mathrm{N}=369)\end{array}$ & & \\
\hline BMI, mean (SD) & $26.0(2.6)$ & $25.9(2.3)$ & $26.0(2.9)$ & $26.1(2.5)$ & & \\
\hline $\begin{array}{l}\text { Average daily minutes } \\
\text { moderate and vigorous } \\
\text { physical activity, mean (SD) }\end{array}$ & $114.9(109.7)$ & $114.8(100.6)$ & $113.8(97.5)$ & $115.5(120.5)$ & & \\
\hline $\begin{array}{l}\text { Average daily energy-intake, } \\
\text { mean (SD) }\end{array}$ & 1,079.0 (448.3) & $1,017.7(434.5)$ & $992.9(460.4)$ & $1,157.6(436.1)$ & & \\
\hline $\begin{array}{l}* * P<0.01 \\
* P<0.05 \\
\text { a, b Values within } \\
\text { Bonferroni correc }\end{array}$ & ow with iden & ter & differ & nined & 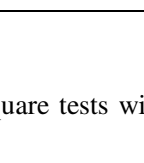 & \\
\hline
\end{tabular}

\section{Intervention use}

The average number of completed sessions was $2.1(\mathrm{SD}=1.9)$ in the video condition and 2.2 $(\mathrm{SD}=2.0)$ in the text condition, but this difference was not statistically significant $(\mathrm{F}=1.55$, $P=$.214). In the analyses stratified by level of education also no significant differences were found in the average number of completed sessions between the text and video condition per educational level: low $(\mathrm{F}=0.04, P=.841)$, medium $(\mathrm{F}=2.47, P=.117)$, and high $(\mathrm{F}=0.23$, $P=$.634) educational level.

Yet, there was a significant difference in use between participants who were allocated to an intervention condition that matched their preferred intervention delivery 
format and those with a mismatch $(\mathrm{F}=4.58 ; P=.033)$. The average number of completed session was $2.2(\mathrm{SD}=2.0)$ among participants with a match, while this was $1.9(\mathrm{SD}=1.9)$ among participants with a mismatch.

\section{Influence of intervention use and (mis)matching intervention delivery format on intervention outcomes}

There were no significant condition*intervention use interaction terms for any of the outcome variables (see Table 2). This implies that intervention use did not have a moderating impact on the intervention outcomes. In addition, we also did not find significant condition*(mis)matching intervention delivery format interaction terms for any of the outcome variables (see Table 2). This implies that the intervention outcomes were not influenced by whether or not participants were allocated to an intervention condition that matched their preferred intervention delivery format. These results were found with both the complete cases as multiple imputation dataset.

Table 2. Results of interactions terms regarding intervention use and (mis)matching intervention delivery format

\begin{tabular}{lcc}
\hline Interaction terms & $\boldsymbol{\beta}$ & $\boldsymbol{P}$ \\
\hline Energy-intake & .06 & .589 \\
Video condition*(mis)match & .06 & .558 \\
Text condition*(mis)match & -.04 & .273 \\
Video condition*intervention use & -.06 & .101 \\
Text condition*intervention use & & \\
BMI & .00 & .952 \\
Video condition*(mis)match & .04 & .467 \\
Text condition*(mis)match & -.09 & .320 \\
Video condition*intervention use & -.08 & .329 \\
Text condition*intervention use & & \\
Physical activity & -.04 & .787 \\
Video condition*(mis)match & -.07 & .590 \\
Text condition*(mis)match & .02 & .826 \\
Video condition*intervention use & -.02 & .809 \\
Text condition*intervention use & & \\
\hline
\end{tabular}

\section{Determinants of intervention use}

The determinant analysis shows that participants with a higher BMI were significantly more likely to use the intervention more often (see Table 3). In addition, participants who felt involved and supported by the intervention (i.e. feelings of relatedness) were also significantly more likely to use the intervention more often. The explained variance of the regression model was $16.0 \%$. 
Table 3. Determinants of intervention use (number of completed sessions) as assessed by multiple linear regression analysis

\begin{tabular}{llc}
\hline Determinants & $\boldsymbol{\beta}$ & $\boldsymbol{P}$ \\
\hline Study condition & .06 & .372 \\
Age & .09 & .228 \\
Gender & .13 & .083 \\
Educational level: low versus medium & .01 & .924 \\
Educational level: low versus high & .09 & .453 \\
BMI & .18 & $.015^{*}$ \\
Average daily minutes moderate and vigorous & .09 & .241 \\
physical activity & & .560 \\
Average daily energy-intake & -.04 & .470 \\
The feedback messages fit to my own situation & -.08 & .880 \\
The feedback messages were understandable & .02 & .275 \\
The feedback messages were useful & .12 & .240 \\
The feedback messages were interesting & -.13 & .495 \\
Overall grade intervention (from 1 to 10) & -.08 & .100 \\
Feelings of autonomy & .15 & $.047^{*}$ \\
Feelings of relatedness & .29 & .898 \\
Feelings of competence & .02 & \\
$* *$ P<0.01 & & \\
\hline$<0.05$ & &
\end{tabular}

\section{Discussion}

\section{Principal findings}

The main aim of this study was to examine how the use and effectiveness of web-based computer-tailored interventions can be improved. For this purpose, we first examined if the use of a web-based computer-tailored obesity prevention intervention can be increased by using videos as delivery format and whether more use was related to better intervention effects. Secondly, we examined if the delivery of intervention content via participants' preferred delivery format can increase intervention use and whether this is related to better intervention outcomes. The final aim of the study was to identify which socio-demographic factors and intervention appreciation variables predict intervention use.

Intervention use (i.e. numbers of completed intervention sessions) declined rapidly over time in both versions of the intervention. Contradicting our hypothesis, the video version was not used more often than the text version, neither by the total study population nor among participants with a low educational level. However, the intervention was used more often among participants who received intervention content via their preferred intervention delivery format. Our results further indicate that more intervention use and a matching intervention delivery format did not result in better intervention effects. In general, the intervention was more likely to be used more often by participants with a high BMI and participants who felt involved and supported by the intervention.

The steep decline in intervention use can possibly be explained by the fact that the intervention consisted of six information-rich sessions which required a high level of active involvement (e.g. making plans and answering questions). This probably was too 
demanding for participants and may have resulted in an overload and premature leaves (Brouwer et al., 2009; Prochaska, 2008; Schulz et al., 2012). Another explanation could be the fact that the most important intervention content was included in the first two sessions. It is possible that the content of these sessions was sufficient for participants to enable them to change their behavior successfully. Hence, not all participants may have had the need to also use the last intervention sessions in which their behavior and weight goals were evaluated. These explanations are confirmed by previous research that has shown that people are primarily interested in a simple comparison of their behavior against the relevant guidelines, whereas there is a lack of interest in behavior change counselling sessions that require a high level of active involvement (Verheijden, Jans, \& Hildebrandt, 2008; Verheijden et al., 2007). As in all web-based interventions, drop-out can also be the cause of technical problems, such as errors on the website and slow video buffering (Booth, Nowson, \& Matters, 2008; Krishnan \& Sitaraman, 2013; Kypri \& McAnally, 2005). A study has, for example, shown that participants will quit an intervention when it takes more than two seconds to load a video, with each incremental delay of one second resulting in a $5.8 \%$ increase in drop-out rate (Krishnan \& Sitaraman, 2013). Finally, although the use of videos and tailoring can be considered sophisticated, recent technical developments, such as gamification and mHealth, may have raised users' expectations of new products (Bert, Giacometti, Gualano, \& Siliquini, 2014; Blohm \& Leimeister, 2013; Kumar et al., 2013; PWC, 2012). Hence, the video intervention possibly did not consist of sufficient innovative and attractive characteristics. In conclusion, these findings imply that still more research is needed into how the use of future web-based computer-tailored interventions can be improved. Until strategies have been identified that can increase intervention use successfully, it is recommended to minimize the number of sessions of future web-based computer-tailored interventions and include the most important information for achieving a successful behavior change in the first sessions. Nevertheless, our results show that the use of web-based computer-tailored interventions can be slightly increased by delivering intervention content via users' preferred intervention delivery format. Although this finding is in line with our hypothesis, a recent similar study has concluded that a matching intervention delivery format does not result in more intervention use (Vandelanotte et al., 2012). Hence, first more research into this is needed before firm recommendations can be made about whether or not future interventions should offer participants a delivery format choice.

Moreover, no support was found for the hypothesis that providing intervention content via videos would result in more intervention use among people with a low educational level in particular. This is an interesting finding since a previous study into the efficacy of this intervention has shown that the video version was appreciated significantly better than the text version (Walthouwer et al., submitted). However, two recent studies that have also concluded that the use of videos as delivery format has no effect on intervention adherence (Stanczyk et al., 2013; Vandelanotte et al., 2012). A possible explanation for the 
fact that a better appreciation of the video version has not resulted in more intervention use is that appreciation is also influenced by many factors other than delivery format, such as the usability of an intervention and participants' motivation to change (Brouwer et al., 2009).

The fact that there was no relationship between intervention use and intervention outcomes can possibly also explain why allocation to a matching intervention delivery format did not result in better intervention outcomes despite that this increased intervention use. This finding is further in line with a recent study that has also concluded that the provision of intervention content via a matching delivery format does not result in better intervention outcomes (Vandelanotte et al., 2012). In addition, more intervention use has possibly also not result in better intervention outcomes since the most important information relevant for achieving a successful behavior change was included in the first two intervention sessions and only few participants had completed the whole intervention. It has further been suggested that the relationship between intervention use and health outcomes is curvilinear instead of linear, implying that there is a saturation point after which no further benefit will be obtained (Donkin et al., 2011). More is not always better and sometimes increasing requirements for participants can even have iatrogenic effects, such as a lowered engagement (Glasgow et al., 2007). However, there is also evidence that people need to be exposed to educational content multiple times before intervention effects can be expected (An et al., 2006; Brug, Glanz, van Assema, Kok, \& van Breukelen, 1998; Couper et al., 2010; Dijkstra, de Vries, Roijackers, \& van Breukelen, 1998; Wantland et al., 2004). Prolonged intervention use is, for example, necessary for learning and practicing skills over time. Because of these mixed findings, future research is needed to identify what the optimum number of intervention sessions is in terms of maximizing use and effects.

Finally, the regression analysis concerning the determinants of intervention use also provides indications about how intervention use can be improved. Our results, for example, show that participants with a high BMI and participants who felt involved and supported by the intervention (i.e. feelings of relatedness) were more likely to use the intervention more often. Hence, a potential way to increase intervention use is by creating feelings of relatedness. According to the SDT, creating feelings of relatedness will also result in an increased intrinsic motivation to change and this will make successful behavior changes more likely (Deci \& Ryan, 2011). Based on a previous study into the intervention, it can be concluded that the perceived relatedness can be increased via the inclusion of video messages since the video version was evaluated significantly better on feelings of relatedness compared to the text version (Walthouwer et al., submitted). Possibly, participants feel more involved and supported by a video delivery format since in videos a person is actually talking to them and because it is easier to show empathy via spoken words. In addition, participants with a high BMI were probably more likely to use the intervention more often because they needed more help with changing their behavior 
successfully compared to, for example, participants with a healthy BMI who may have had no direct need to change.

\section{Strengths and limitations}

Our study is characterized by several limitations. First, intervention use was assessed by the number of completed sessions. Although it has been suggested that there is a high correlation between number of completed sessions and time spend at the intervention (Wangberg et al., 2008), this does not give any information about the exposure to and engagement with the intervention content. Other measures, such as use of specific pages and amount of information read, may give a better indication of actual intervention use (Brouwer et al., 2010; Danaher \& Seeley, 2009). Hence, future studies examining the use of web-based computer-tailored interventions should include a more extensive measurement of intervention use (Bennett \& Glasgow, 2009; Brouwer et al., 2011). A second limitation concerns the measurement of preferred intervention delivery format. In contrast to directly asking participants for their preference, it has been suggested that it may be better to assess the preference strength on a scale ranging from low to high (Swift, Callahan, \& Vollmer, 2011). Third, in order to be able to draw clear conclusions about the influence of (mis)matching intervention delivery format, it would have been better to first stratify for intervention delivery format preference before randomizing people to study conditions. The current procedure thus limits the strengths of the conclusions.

Despite these limitations and the fact that we did not find support for all of our hypotheses, this study provides a valuable contribution to the required research into this area. An important strength is, for example, that we used a relatively new strategy (i.e. use of tailored videos) to examine if the use of web-based computer-tailored interventions can be improved among people with a low educational level in particular. Another strength is the fact that the analyses with the multiple imputation data have resulted in exactly the same findings as the analyses with the complete cases data.

\section{Conclusions}

The use of the web-based computer-tailored obesity prevention intervention declined sharply per session and the provision of intervention content via videos did not increase its use, regardless of participants' educational level. This finding and the fact that more intervention use did not result in better intervention outcomes implies that an intervention does not have to consist of many information-rich sessions. Possibly, it is sufficient to develop only two sessions that include the most important information that is necessary for achieving a successful behavior change. However, since only few participants had completed three or more sessions, first more research is needed into the optimum number of intervention sessions in terms of maximizing use and effects. In addition, our study also shows that intervention use can be increased by the provision of intervention content via participants' preferred intervention delivery format. However, since this did not lead to 
better intervention outcomes, first more research is needed to examine if it is cost-effective to include a delivery format preference-based tailoring option. Finally, it can be concluded that intervention use can be increased by ensuring that participants feel involved and supported by the intervention. 


\title{
CHAPTER 7
}

\begin{abstract}
A qualitative study on barriers and facilitators to the implementation of a web-based computer-tailored obesity prevention intervention by occupational health centers
\end{abstract}

Submitted as: Walthouwer, M., Oenema, A., Soetens, K., Lechner, L., \& de Vries, H. A qualitative study on barriers and facilitators to the implementation of a web-based computer-tailored intervention by occupational health centers. 


\begin{abstract}
Background: The aim of this qualitative study was to identify barriers and facilitators to the adoption and particularly the implementation of a web-based computer-tailored obesity prevention intervention by occupational health centers.

Methods: Participants were directors of Dutch occupational health centers who had adopted and implemented the intervention for the corresponding efficacy study $(\mathrm{N}=8)$ as well as non-adopters $(\mathrm{N}=12)$. Individual semi-structured interviews were carried out to study barriers and facilitators related to the intervention, the user, the organization, and the sociopolitical environment. All interviews were carried out by telephone, audio-recorded, and transcribed verbatim. The transcripts were analyzed using a directed content approach and coded by two persons.

Results: There were important differences in perceptions between adopters and nonadopters, particularly on barriers and facilitators related to the intervention and the personal beliefs of the implementer. The relative advantages of the intervention were considered to be most important. Participants also indicated that their personal attitudinal and selfefficacy beliefs influenced their implementation efforts. Regarding the organization, the possibilities to increase profits and integrate the intervention within the organization were considered to be important facilitators for the implementation. Participants mentioned few implementation barriers and facilitators related to the socio-political environment.

Conclusions: Strategies to improve the implementation of web-based computer-tailored interventions by occupational health centers should be tailored to implementers' unique perceptions and particularly address the perceived (dis)advantages of the intervention, attitudinal and self-efficacy beliefs, and the potential to increase organizations' profits and competitiveness.
\end{abstract}

Keywords: occupational health centers, qualitative study, implementation, determinants, web-based intervention 


\section{Background}

Worksites are increasingly interested in improving employee health with the purpose to increase sustainable work and productivity as well as to decrease health care costs (Hughes, Patrick, Hannon, Harris, \& Ghosh, 2011). There are many different strategies that can be used to provide employees with health promotion programs. One promising implementation strategy is providing such programs in collaboration with occupational health centers. These organizations have the objective to provide both cure and care for employees including, for example, performing health screenings and providing health promotion programs (Hulshof, Verbeek, van Dijk, Van der Weide, \& Braam, 1999). Hence, occupational health centers have the potential to reach many employees in a relatively easy way.

Nevertheless, broad scale and successful implementation of worksite health promotion programs in daily practice often fails and this limits their potential impact (Durlak \& Dupre, 2008; Grimshaw, Eccles, \& Tetroe, 2004; Grol \& Grimshaw, 2003; Grol $\&$ Wensing, 2004). Before an intervention can be implemented, an organization should first decide to adopt the intervention. Adoption is defined as the decision to make full use of an intervention, while implementation occurs when an individual (or other decision-making unit) puts a new idea (i.e. intervention) into use (Rogers, 2010; Weiner, Lewis, \& Linnan, 2009). For both phases, different barriers and facilitators may be of relevance. Adoption of an intervention is the first necessary step but the quality of implementation determines the health benefits of employees (Durlak \& Dupre, 2008; Klein \& Sorra, 1996). Many health care organizations such as occupational health centers often adopt interventions with great anticipation, yet to fail to realize an optimal implementation (Helfrich, Weiner, McKinney, $\&$ Minasian, 2007). Proper implementation frequently proves to be too challenging, timeconsuming, and costly for many of these organizations (Shortell, Bennett, \& Byck, 1998).

It has been suggested that web-based interventions may improve implementation rates as these interventions are easy to implement. Web-based interventions are provided to users via a website on the Internet and can be used with, for example, computers or mobile devices such as tablets and mobile phones. These interventions further have the potential to provide information that is tailored to the unique perceptions of the user; these interventions are defined as web-based computer-tailored interventions (Eysenbach, 2011). In the last decade attention for these interventions has increased because they have the potential to reach many people with individualized information in an easy way against low costs (Cobiac et al., 2009; Oenema et al., 2001). These interventions further do not necessitate high levels of involvement of health professionals since they only have to refer clients to these interventions, which should make implementation easy and time-saving. Nevertheless, implementation rates of web-based interventions by health professionals are relatively low (Bennett \& Glasgow, 2009). Health professionals are reluctant to accept and utilize new information and communication technologies (ICT) (Schaper \& Pervan, 2007). Hence, there is a need to improve the implementation of future web-based computer- 
tailored interventions by, for example, occupational health centers. It has been suggested that this can be done most effectively by addressing the most salient barriers and facilitators in implementation strategies (Murray et al., 2011; Sorensen \& Barbeau, 2004).

A comprehensive study on barriers and facilitators to adoption and implementation of ICT by occupational therapists has demonstrated that personal beliefs and convenience of the intervention are important facilitators that should be targeted with implementation strategies (Schaper \& Pervan, 2007). One study among managers of occupational health centers has further shown that the psychosocial domain and work environment influence the implementation of interventions aimed to tackle ill-health (Whysall, Haslam, \& Haslam, 2006). Another study has demonstrated that the implementation of an occupational health guideline by occupational physicians is mainly influenced by barriers and facilitators related to their own role, such as (lack of) support from colleagues and limited knowledge, as well as the relative advantages of the innovation itself (Verweij et al., 2012). However, although these studies provide indications about how the implementation of interventions by occupational health professionals can be improved, there is insufficient evidence with respect to the barriers and facilitators to the implementation of in particular web-based computer-tailored interventions by occupational health centers.

To gain a better understanding of the barriers and facilitators to the adoption and particularly the implementation of a web-based computer-tailored intervention by occupational health centers in the Netherlands, we have carried out the present exploratory qualitative study. In the Netherlands, employers are required by law to ensure support on occupational health and absenteeism. For this purpose, companies can hire commercial companies such as occupational health centers. Within these organizations, many types of people are employed, including occupational physicians, physical therapists, dieticians, and managers. In smaller occupational health centers, the decision maker (director) often also is one of the implementers.

For this study we used a theoretical framework (Fleuren, Wiefferink, \& Paulussen, 2004) that has integrated various models, such as the Theory of Planned Behavior (Ajzen, 1991) and Rogers's Diffusion of Innovation Theory (Rogers, 2010). This framework has been used successfully in the past to, for example, explore the determinants influencing the implementation of new technologies among nursing staff (de Veer, Fleuren, Bekkema, \& Francke, 2011). According to this framework, four different types of factors (i.e. determinants) can positively or negatively influence the innovation process (i.e. dissemination, adoption, implementation, and continuation) (see Figure 1). Although this model can be used to study factors related to all phases in the innovation process (from dissemination to continuation), this study focused on the barriers and facilitators related to adoption and implementation only. The framework firstly demonstrates that barriers and facilitators related to the intervention itself influence implementation, such as the perceived complexity of the intervention or the relative benefits it offers compared to existing interventions (Damschroder et al., 2009; de Veer et al., 2011; Fleuren et al., 2004; Francke, 
Smit, de Veer, \& Mistiaen, 2008; Peels et al., 2014; Verweij et al., 2012). Second, characteristics of the user (i.e. the adopter or implementer) can also have a significant influence on implementation. The implementation of an intervention will, for example, be facilitated when the user has sufficient skills, positive outcome expectations, and a high self-efficacy with regard to implementing the intervention (Damschroder et al., 2009; Fleuren et al., 2004; Peels et al., 2014; Smit, de Vries, \& Hoving, 2013; Verweij et al., 2012). Third, barriers and facilitators related to the organization can also influence the success of implementation, such as staff capacity, organizational climate, and management support (de Veer et al., 2011; Fleuren et al., 2004; Murray et al., 2011; Verweij et al., 2012). Finally, the socio-political environment has an influence on implementation efforts as well, including governmental policy, societal developments (e.g. economic crisis), and competition within the work area (Fleuren et al., 2004; Verweij et al., 2012).

In this study we have examined which barriers and facilitators influence the adoption and particularly the implementation of a web-based computer-tailored obesity prevention intervention by Dutch occupational health centers. The results of this study can be used to develop more appropriate and effective implementation strategies.

Figure 1. Theoretical framework of Fleuren and colleagues (2004): Determinants of Innovation Processes

Innovation determinants

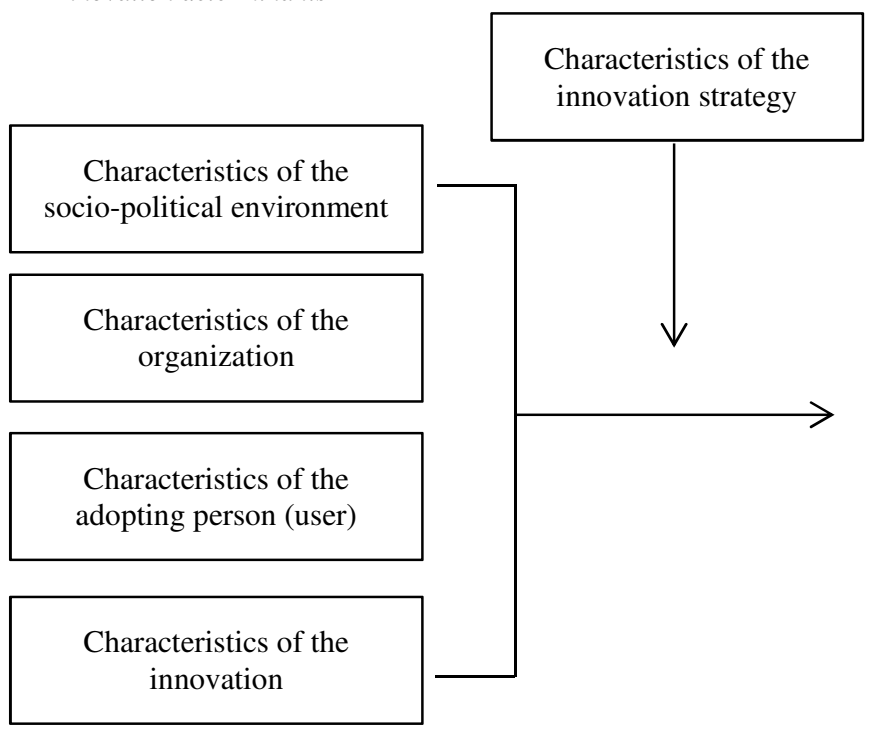

Innovation process

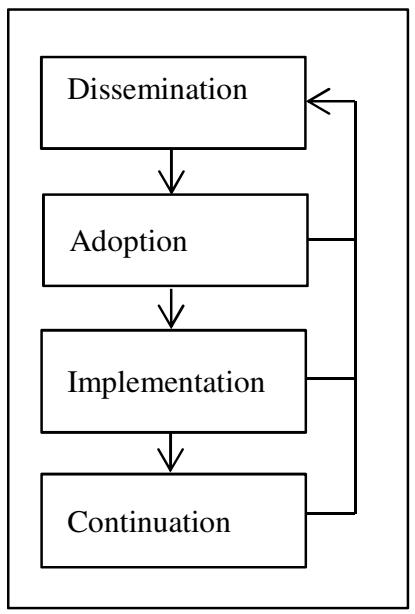

\section{Methods}

The Ethics Committee of the Open University Heerlen reviewed the study protocol and decided that no approval of the medical ethics committee was required. Relevant aspects of this study are reported following the Consolidated Criteria for Reporting Qualitative Research (COREQ) (Tong, Sainsbury, \& Craig, 2007). 


\section{Respondents and procedure}

This qualitative study was carried out as part of a larger research project: a randomized controlled trial (RCT) to test the effects of two versions of a web-based computer-tailored obesity prevention intervention (named 'Weight in the Balance') addressing dietary intake, physical activity, and BMI among Dutch adults with a healthy weight or limited overweight (Walthouwer et al., 2013). To recruit participants for this RCT, 41 Dutch occupational health centers were approached by e-mail or telephone to inquire if they were willing to recruit participants and thereby implement the intervention (and study). Eventually, 16 of these 41 occupational health centers had made a positive adoption decision and agreed with participation in the intervention study. To implement the intervention, these organizations were asked to provide information about the intervention and offer a flyer about the intervention study to their clients during medical screenings. Unfortunately, this strategy turned out to be unsuccessful and yielded only about one hundred participants. Postimplementation, thus after the end of the RCT, the present qualitative study was conducted.

To recruit participants for this study, we approached the directors of the 16 occupational health centers who had adopted and implemented our web-based computertailored obesity prevention intervention as well as directors who had refused this (i.e. nonadopters), as it is important to include the opinion of both users and non-users (Fleuren et al., 2004). Eventually, twenty semi-structured telephone interviews were held with directors of occupational health centers (response $=58.8 \%$ ). Ten of these directors indicated that they were not fully involved in the actual implementation of interventions and mostly had a managing director role, whereas the other ten directors were both manager and occupational physician and accordingly also actually implemented interventions themselves. Concurrent data analysis showed that data saturation was reached after these twenty interviews. The interviews were carried out by a female (KS) and a male researcher (MJLW). Both researchers had experience with qualitative research and a master's degree in health promotion. Interviews lasted from 18 to 41 minutes (mean=26 minutes). All participants gave their verbal informed consent via the telephone prior to the interview.

\section{Measures}

The semi-structured interviews were carried out using an interview guide, which was developed based on the theoretical framework described before (Fleuren et al., 2004). The interview guide was tested in two pilot interviews and adapted where needed. The interview guide consisted of ten open-ended questions, which were augmented with prompts and examples. First, several demographics were assessed, including gender, educational level, age, and years of relevant work experience (i.e. experience in the field or in related fields). The main interview started with a question on why participants had agreed or refused to participate in our RCT (i.e. adoption decision). Subsequently, implementation barriers and facilitators related to the intervention, the implementer, the organization, and the sociopolitical environment were assessed using one open-ended question per factor. Participants 
were asked to elaborate on their answer and to think of impeding as well as facilitating factors. Prompts were used to facilitate elaboration on each topic. First, barriers and facilitators related to the intervention were assessed by asking participants: 'Which intervention characteristics are for you of influence on the implementation of an intervention such as Weight in the Balance?'. Next, barriers and facilitators related to the participants' own beliefs were assessed with the question: 'What are important reasons for you to implement an intervention such as Weight in the Balance?'. Subsequently, barriers and facilitators related to the organization were assessed by posing the following question: 'Which factors within your organization are of influence on the implementation of an intervention such as Weight in the Balance?'. Finally, barriers and facilitators related to the socio-political environment were assessed by asking participants: 'Which factors within the socio-political environment are of influence on the implementation of an intervention such as Weight in the Balance?'. It should be noted that the implementation questions were hypothetical for the participants who had not adopted the intervention, since these participants had no actual experience with implementing the intervention.

\section{Data analysis}

All interviews were audio-recorded and transcribed verbatim. Transcripts were not returned to participants for comments. We used a directed content approach to the data analysis. With a directed approach, the analysis starts with a theory or relevant research findings as guidance for initial codes. A directed approach is appropriate if existing theory and earlier research about a phenomenon (e.g. the implementation of an intervention) are incomplete or would benefit from further description (Hsieh \& Shannon, 2005). In accordance with this approach, a code book was developed using the model of Fleuren and colleagues (2004) and the results of prior research (Chaudoir, Dugan, \& Barr, 2013; Hughes et al., 2011; Verweij et al., 2012; Wierenga et al., 2013). The transcripts were independently coded by the two interviewers (MJLW and KS) using standard text processing software. During this process, additional codes were added to the initial coding scheme. The inter-rater reliability between the coding of the two coders was calculated using a crosstab analysis with Cohen's Kappa. The agreement was $0.83(P=.000)$, which implies a very high agreement (Landis \& Koch, 1977). Disagreement in coding was discussed until consensus was reached.

\section{Results}

The results are presented per factor of the theoretical framework of Fleuren and colleagues (2004) using quotes to clarify answers. Table 1 and 2 further provide an overview of the most relevant themes (i.e. barriers and facilitators), separately for participants of occupational health centers who had adopted (and accordingly implemented) the intervention and those who had not. 


\section{Sample}

The mean age of the twenty interviewees was $44.3(\mathrm{SD}=7.1)$ and they all had a high educational level. Eight participants were female (40.0\%) and the average working experience was 13.8 years $(\mathrm{SD}=6.3)$. Further, eight of the twenty participants $(40.0 \%)$ came from occupational health centers that had adopted and implemented our intervention (i.e. adopters).

\section{Barriers and facilitators to adoption}

The twelve participants who had decided not to adopt the intervention (i.e. not participate in the RCT) named several reasons for this decision (see Table 1). The most frequently mentioned reasons were that the implementation would be too time-consuming and a lack of resources such as money and support staff. Seven participants also indicated that the intervention would be competitive with their own interventions that worksites have to pay for and they were therefore afraid that offering our intervention would decrease their profits. A less frequently mentioned reason for non-adoption was that the occupational health center was not ready for offering web-based computer-tailored interventions. One participant, for example, said: 'the ICT in my organization is outdated and we are unable to offer interventions via the Internet. This will require a large update of our ICT system, which I currently cannot afford'.

The eight persons who had decided to adopt and accordingly implement the intervention indicated that they mainly made this decision because the intervention was easily accessible and did not cost money. Another frequently mentioned reason for this decision was that the intervention fits well with occupational health centers own activities and services. One participant, for example, highlighted: 'Our organization is more likely to adopt an intervention when it can be integrated within our own health services. This is important because our clients should have the idea that they are using our own intervention'. Finally, three participants indicated that their adoption decision was based on the fact that they like to be part of innovative research projects and are interested in new developments in their work area.

Table 1. Reasons for (non-)adoption, separately for adopting and non-adopting occupational health centers

\begin{tabular}{ll}
\hline & \multicolumn{1}{c}{ Reasons for adoption $(\mathbf{N}$ times mentioned, \%) } \\
\cline { 2 - 2 } Adopting occupational health centers $(\mathbf{N}=\mathbf{8})$ & Non-adopting occupational health centers $(\mathbf{N}=\mathbf{1 2})$ \\
\hline - Intervention does not cost money $(5,62.5 \%)$ & - Time-consuming and lack of resources $(8,66.7 \%)$ \\
- Intervention is easily accessible $(5,62.5 \%)$ & - Competitive with own interventions $(7,58.3 \%)$ \\
- Intervention fits with activities $(4,50.0 \%)$ & - Afraid of decrease of profits $(5,41.7 \%)$ \\
- Intervention is complementary to own health services & - Organization not ready for eHealth $(3,25.0 \%)$ \\
$(4,50.0 \%)$ & \\
- Interested in innovative project/new developments $(3$, \\
$37.5 \%)$
\end{tabular}




\section{Barriers and facilitators to implementation}

The intervention

As can be noted from Table 2, the occupational health centers that had adopted the intervention $(\mathrm{N}=8)$ indicated that implementation of a web-based computer-tailored intervention (such as Weight in the Balance) had many important relative advantages. About half of the participants perceived advantages such as the intervention is online and can be used whenever and where people prefer, the personalized approach of the intervention, and the large amount of freedom in the intervention. These facilitators were highlighted by one participant, who said: 'A web-based intervention can be used at home, at a time when this is most suitable for our clients. That is an important advantage. Another advantage is the fact that the intervention also provides tailored information, which ensures that participants may still perceive to receive an individualized treatment'. Six interviewees also indicated that they were more likely to implement the intervention because they perceived Weight in the Balance as an actual innovation. Furthermore, three participants preferred to implement an intervention that is developed by a University. On the contrary, six participants of the twelve non-adopting occupational health centers indicated that they would not implement the intervention because they found it an important disadvantage that the intervention was fully online and did not consist of a personal face-to-face component. In particular these non-adopters doubted if providing information via the Internet only would be sufficient to motivate people, achieve behavior change, and bind clients to an intervention. In addition, compatibility of an intervention was considered to be important as well. Eight participants, for example, indicated that they were more inclined to implement the intervention when it would be possible to integrate it with their own interventions and ICT.

As presented in Table 2, complexity and trialability were regarded as less important for the implementation of the web-based computer-tailored intervention. Six interviewees raised concerns about the complexity of such interventions for both themselves and their clients, especially for the elderly. One participant, for example, said: 'To implement a web-based computer-tailored intervention successfully, it should be easy in use, especially because the Internet can already be complex for some people. Every component of the intervention needs to be clear, including the navigation through the intervention, the log-in procedure, and the information'. Regarding trialability, five participants indicated that they were more inclined to implement the intervention when they could try it in advance before offering it to their clients.

\section{The implementer}

Table 2 shows that the implementation of web-based computer-tailored interventions such as Weight in the Balance is influenced by implementers' own beliefs, in particular their attitude and self-efficacy and to a lesser extent their perceived social norm. Overall, there 
were conflicting beliefs between participants from occupational health centers who had adopted (and accordingly implemented) the intervention and those who had not.

In particular the participants who had adopted the intervention indicated that the implementation of the intervention had important advantages, including the possibility to broaden their offer of health promotion programs, attract new clients, and improve the quality of their health services. Participants from the non-adopting occupational health centers mentioned much less personal advantages. Interestingly, only four participants explicitly stated that achieving health benefits for their clients was an important reason to implement the intervention. In addition, five participants of the non-adopting organizations indicated that the RCT related to the intervention would be an important barrier to the implementation as the trial required additional efforts for the participants (e.g. filling out a baseline and follow-up questionnaire) and included a control condition that would not receive any intervention. Inversely, the trial was considered to be a facilitating factor by four participants of the adopting occupational health centers. These participants indicated that they had implemented the intervention and the corresponding trial to get an overview of the study results and improve their own knowledge. Furthermore, several participants doubted if a web-based computer-tailored intervention can actually bind clients and were therefore less inclined to implement the intervention.

Participants also named several barriers related to their self-efficacy. Especially those who had not adopted the intervention indicated that they lacked knowledge and skills regarding eHealth and therefore did not feel comfortable to implement a web-based computer-tailored intervention. In addition, lack of time was also indicated as a barrier to implementation by five participants. One participant, for example, said 'a web-based computer-tailored intervention, such as Weight in the Balance, has to be simple in nature and straightforward. If I have to explain how the intervention should be used, the implementation will become too difficult and too time-consuming for me'.

Finally, about half of the participants indicated that the opinion of their colleagues was important as well. Six participants, for example, indicated that they would be more inclined to implement a new intervention when their colleagues would like to offer it as well. In addition, five participants highlighted that they would only implement the intervention if this would not increase the workload for their colleagues.

\section{The organization}

Participants named various barriers and facilitators within their organization of influence on the implementation of interventions such as Weight in the Balance (see Table 2). Overall, there were no differences in perceptions between the participants of occupational health centers who had adopted the intervention and those who had not. Thirteen participants highlighted that the implementation of a web-based computer-tailored intervention such as Weight in the Balance will be facilitated when this would result in an increased profit for their organization. Several participants actually said that they had decided not to adopt and 
implement Weight in the Balance because this would not increase their profits. Related to this, about half of the participants indicated that the implementation will be facilitated when the intervention would be complementary to their own health services and does not compete with their own interventions. One participant, for example, said 'when a client needs a health promotion program I will of course first offer one of our own services instead of a similar service of an external organization, particularly when I will earn less by offering this external intervention. Hence, the external intervention will always be secondchoice when I have a similar own health service'. About half of the participants also stressed that they were more likely to implement an externally developed intervention when it can be integrated with the organizations' own interventions, protocols, and data systems. One participant, for example, said 'it is very important that an externally developed intervention looks the same as our own interventions. Otherwise, this will be confusing for both our clients and personnel'. A clear communication process and implementation policy were also named by several interviewees as of importance for the implementation. One participant, for example, said 'it is important that I receive all necessary information and know exactly what I have to do. I would further also appreciate it if I would receive regular updates and visits from the project team'. Finally, five participants perceived the implementation as an additional workload and of those three indicated that the implementation would be facilitated when the development team could deliver personnel, such as students, to carry out the implementation.

\section{The socio-political environment}

As can be seen in Table 2, the smallest number of facilitators and barriers were related to the socio-political environment. In addition, there were no differences in answers between the adopting and non-adopting occupational health centers. The majority of the participants indicated that the economy was an important barrier that hindered the implementation of Weight in the Balance. Since the economic recession of 2008, twelve interviewees experienced that clients as well as companies are less interested to engage in worksite health promotion. One participant, for example, said 'many companies want to save money on everything that has no direct effect. They only want to invest money in current problems and pay less attention to things that have an influence on the long-term, such as health checks and health promotion programs'. Seven participants indicated that the offer of competing organizations also has a significant influence on their implementation efforts. These participants, for example, indicated that they always tried to be competitive and offer comparable health services. Almost half of the participants also indicated that societal issues play an important role in their efforts to implement health promotion interventions. These participants, for example, said that obesity is a hot topic and that they are often approached about this by companies. Hence, obesity received a lot of attention within their organization. One participant, for example, said 'our offer of health services is largely determined by the demand of our customers. Currently, many companies pay most attention 
to obesity and we therefore have multiple services targeted at the treatment and prevention of obesity. Focusing on hot topics, such as obesity, further is the most effective way for increasing our profits'. Several participants also indicated that because of the increasing societal focus on cost-effective health care, they were increasingly interested in implementing web-based interventions. Finally, three interviewees indicated that they thought that the implementation of worksite health promotion interventions in general would be facilitated if the Dutch law would make it mandatory for companies to offer such programs. 
Table 2. Reasons for implementation, separately for adopting and non-adopting occupational health centers

\begin{tabular}{|c|c|c|c|c|}
\hline & \multicolumn{2}{|l|}{ Facilitating (N times mentioned, \% $)^{a}$} & \multicolumn{2}{|c|}{ 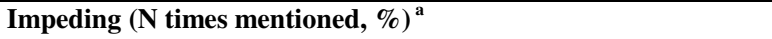 } \\
\hline & $\begin{array}{l}\text { Adopting occupational health } \\
\text { centers }(\mathrm{N}=8)\end{array}$ & $\begin{array}{l}\text { Non-adopting occupational } \\
\text { health centers }(\mathrm{N}=12)\end{array}$ & $\begin{array}{l}\text { Adopting occupational health } \\
\text { centers }(\mathrm{N}=8)\end{array}$ & $\begin{array}{l}\text { Non-adopting occupational } \\
\text { health centers }(\mathrm{N}=12)\end{array}$ \\
\hline \multicolumn{5}{|l|}{ Characteristics of the intervention } \\
\hline \multirow[t]{7}{*}{ Relative advantage/disadvantage } & - Intervention is online $(7,87.5 \%)$ & - Intervention is online $(2,16.7 \%)$ & $\begin{array}{l}\text { - Intervention is fully online, no } \\
\text { personal contact }(1,12.5 \%)\end{array}$ & $\begin{array}{l}\text { - Intervention is fully online, no } \\
\text { personal contact }(6,50.0 \%)\end{array}$ \\
\hline & $\begin{array}{l}\text { - Personalized approach of } \\
\text { intervention }(4,50.0 \%)\end{array}$ & $\begin{array}{l}\text { - Personalized approach of } \\
\text { intervention }(2,16.7 \%)\end{array}$ & & \\
\hline & $\begin{array}{l}\text { - Lot of freedom in intervention (4, } \\
50.0 \%)\end{array}$ & & & \\
\hline & $\begin{array}{l}\text { - Intervention is actual innovation (3, } \\
37.5 \%)\end{array}$ & $\begin{array}{l}\text { - Intervention is actual innovation } \\
(3,25.0 \%)\end{array}$ & & \\
\hline & - Intervention is developed by & - Intervention is developed by & & \\
\hline & University $(2,25.0 \%)$ & University $(1,8.3 \%)$ & & \\
\hline & & & & $\begin{array}{l}\text { - A web-based intervention is } \\
\text { ineffective }(7,58.3 \%)\end{array}$ \\
\hline Compatibility & $\begin{array}{l}\text { - Integration possibilities with own } \\
\text { services }(3,37.5 \%)\end{array}$ & $\begin{array}{l}\text { - Integration possibilities with } \\
\text { own services }(5,41.7 \%)\end{array}$ & & \\
\hline Complexity & & & $\begin{array}{l}\text { - A web-based intervention is } \\
\text { complex }(3,37.5 \%)\end{array}$ & $\begin{array}{l}\text { - A web-based intervention is } \\
\text { complex }(3,25.0 \%)\end{array}$ \\
\hline Trialability & $\begin{array}{l}\text { - Possibility to try intervention }(2 \text {, } \\
25.0 \%)\end{array}$ & $\begin{array}{l}\text { - Possibility to try intervention (3, } \\
25.0 \%)\end{array}$ & & \\
\hline \multicolumn{5}{|l|}{ Characteristics of the implementer } \\
\hline \multirow[t]{3}{*}{ Attitude } & $\begin{array}{l}\text { - Increase offer of interventions and } \\
\text { attract new clients }(7,87.5 \%) \\
\text { - Improve quality of health services } \\
(5,62.5 \%)\end{array}$ & $\begin{array}{l}\text { - Increase offer of interventions } \\
\text { and attract new clients }(4,33.3 \%) \\
\text { - Improve quality of health } \\
\text { services }(2,16.7 \%)\end{array}$ & & \\
\hline & $\begin{array}{l}\text { - Research related to intervention }(6 \text {, } \\
75.0 \%)\end{array}$ & $\begin{array}{l}\text { - Research related to intervention } \\
(2,16.7 \%)\end{array}$ & & $\begin{array}{l}\text { - Research requires too much } \\
\text { effort }(5,41.7 \%)\end{array}$ \\
\hline & $\begin{array}{l}\text { - Possible health benefits for clients } \\
(3,37.5 \%)\end{array}$ & $\begin{array}{l}\text { - Possible health benefits for } \\
\text { clients }(1,8.3 \%)\end{array}$ & & \\
\hline
\end{tabular}


Self-efficacy

Social norm

- Participation of colleagues (2, $25.0 \%)$

- Participation of colleagues (4, $33.3 \%)$

\section{Characteristics of the}

\section{organization}

- Economic benefit: increase of profits
$(6,75.0 \%)$
- Complementary to own services $(6$,
$75.0 \%)$
- Clear policy for implementation of
intervention $(3,37.5 \%)$

Characteristics of the sociopolitical environment
- Economic benefit: increase of profits $(7,58.3 \%)$

- Complementary to own services $(8,66.7 \%)$

- Clear policy for implementation of intervention $(5,41.7 \%)$

- Assistance during

implementation $(3,25.0 \%)$
- Difficulties to bind participants with eHealth $(1,12.5 \%)$

- Lack of skills regarding eHealth

$(2,25.0 \%)$

- Insufficient time to implement

intervention $(1,12.5 \%)$

- Increased workload for colleagues $(2,25.0 \%)$
- Difficulties to bind participants with eHealth $(5,41.7 \%)$

- Lack of skills regarding eHealth $(7,58.3 \%)$

- Insufficient time to implement intervention $(4,33.3 \%)$

- Increased workload for colleagues $(3,25.0 \%)$
- Additional workload for colleagues $(4,33.3 \%)$
- Offer of competing organizations (4, $50.0 \%$ )

- Societal issues: hot topic $(4,50.0 \%)$

- Laws and regulations $(1,12.5 \%)$
- Offer of competing organizations $(3,25.0 \%)$

- Societal issues: hot topic (5,

41.7\%)

- Laws and regulations (2, 16.7\%)
- Additional workload for colleagues $(1,12.5 \%)$ 


\section{Discussion}

The aim of this exploratory qualitative study was to identify barriers and facilitators to the adoption and particularly the implementation of a web-based computer-tailored intervention by Dutch occupational health centers.

Our study shows that most barriers and facilitators were related to the personal beliefs of the implementer (i.e. user) and the characteristics of the intervention. Participants mentioned less barriers and facilitators related to the organization and the least related to the socio-political environment. Interestingly, there were large differences in answers between the participants of occupational health centers who had adopted and implemented the intervention and those who had not: the directors of the adopting organizations had a much more positive attitude and a more positive perception of the characteristics of the intervention than the directors of the non-adopting organizations.

Despite the fact that health care organizations are increasingly interested in webbased interventions, adoption rates are still low worldwide (Li, Land, \& Ray, 2008). In line with a recent review about reasons for adopting eHealth (Li, Talaei-Khoei, Seale, Ray, \& MacIntyre, 2013), our study shows that adoption is particularly determined by the perceived effort it takes to implement the intervention, accessibility of an intervention for employees, complementarity to organizations' own health services, and possibilities to increase profits. It is, however, questionable whether targeting these beliefs will actually increase adoption rates, since there are large differences in perception of the same intervention. Directors of occupational health centers who had decided to implement the intervention, for example, indicated that it is easy to implement a web-based computertailored intervention, while the non-adopters indicated that the implementation would be too time-consuming and too complex. These differences in perception suggest that having one standard adoption strategy may not be suitable for every organization. Possibly, these strategies will be more effective when they are tailored to the unique perceptions of the person who is responsible for the adoption and implementation. This, for example, offers the possibility to correct misperceptions (which were common among non-adopters in our study) and provide counterarguments for barriers that are of relevance for a specific person.

Our study further shows that the actual implementation of a web-based computertailored intervention by occupational health centers is mainly influenced by barriers and facilitators related to the intervention and the beliefs of the implementers (i.e. users). In particular the perceived advantages of this type of intervention appeared to facilitate implementation, while the perceived disadvantages impeded implementation. In line with previous research (Damschroder et al., 2009; Fleuren et al., 2004; Greenhalgh, Robert, Macfarlane, Bate, \& Kyriakidou, 2004; Helmink, Kremers, van Boekel, van Brussel-Visser, \& de Vries, 2012; Koppelaar, Knibbe, Miedema, \& Burdorf, 2009), participants mentioned many personal advantages for implementing the intervention, such as the possibility to attract new clients and improving their health services. There, however, were important differences in perceptions of these barriers and facilitators between directors of 
occupational health centers who had adopted the intervention and those who had not. In particular the non-adopters perceived many disadvantages hindering implementation of a web-based computer-tailored intervention, such as the difficulty to bind participants with this type of intervention and the fact that the intervention was fully online. As suggested before, a tailored implementation strategy may therefore be most suitable to improve the implementation of web-based computer-tailored interventions by occupational health centers. This offers the possibility to focus implementation strategies on the most salient beliefs toward implementing an intervention and take people's unique (mis)perceptions into account. This suggestion is strengthened by previous research among general practitioners that has shown that the use of tailored implementation strategies leads to a better implementation than having one general implementation strategy (Baker, Reddish, Robertson, Hearnshaw, \& Jones, 2001; Grol \& Grimshaw, 2003).

The large differences between the adopting and non-adopting occupational health centers can possibly be explained by the fact that they could belong to a different adopter category (Rogers, 2010). The organizations that had adopted and implemented Weight in the Balance could be seen as innovators or early adopters: people who are interested in new ideas and able to cope with uncertainty about an innovation at the time of adoption. These people are in general more willing to use (i.e. implement) a new innovation (such as a webbased computer-tailored intervention). On the contrary, the organizations that had refused to implement the intervention could be part of the later adopter categories, such as the late majority or laggards. These people are usually more skeptical about new developments and often adopt an intervention because of economic necessity (Rogers, 2010). When a new innovation is launched, this last group of people often perceives many barriers for not using it. Because early and late adopters generally differ in personality variables and communication behavior, this again implies that the use of a tailored implementation strategy may be more suitable than having one general strategy (Rogers, 2010).

Another interesting finding is that only one quarter of the participants indicated that they would implement a web-based computer-tailored intervention in order to achieve health benefits for their clients. For nearly all occupational health centers, an increased profit for the organization as a result of implementing the intervention was eventually the predominant motivation. Even though it may sound counterintuitive to health promoters, this may indicate that future implementation strategies should pay explicit attention to the potential to increase profits and to a lesser extent the possibility to achieve health benefits for clients. Other organizational aspects that have to be taken into account in these strategies are the possibility to integrate the intervention within the organization and the potential to have a unique intervention that is complementary to occupational health centers' own offer.

Finally, our results show that few implementation barriers and facilitators were related to the socio-political environment. This contradicts previous implementation research that has shown that the socio-political environment includes many barriers and 
facilitators influencing the implementation of health promotion programs (Fleuren et al., 2004; Verweij et al., 2012). It is, however, possible that the socio-political environment is just less relevant for web-based computer-tailored interventions since several studies in other settings have shown that the implementation of these interventions is mostly influenced by factors related to the implementer's own beliefs and the characteristics of the intervention (Mair et al., 2012; Murray et al., 2011; Schers, Wensing, Huijsmans, van Tulder, \& Grol, 2001; Smit, de Vries, et al., 2013). Nevertheless, future implementation strategies will still benefit from paying attention to the socio-political environment since our results suggest that the implementation of web-based computer-tailored interventions can be facilitated further when they are considered to increase organizations' competitiveness and fit with societal issues.

In conclusion, several recommendations for practice and future research can be made. First, when developing implementation strategies, it is crucial to analyze and target the most salient barriers and facilitators to the implementation of web-based computertailored interventions. Our results indicate that these strategies should take into account that the perception of the implementer is more important than the objectively valued characteristics of an intervention. For example, although it seems reasonable that the implementation of a web-based computer-tailored intervention does not require much time, implementers can still perceive this as too time-consuming. This discrepancy should be addressed in future implementation strategies by tailoring them to the unique perceptions of the implementer. These strategies should in particular focus on the perceived advantages of the intervention, the attitudinal and self-efficacy beliefs of the implementer, and the potential to increase organizations' profits and competitiveness. However, the identified barriers and facilitators were only described in qualitative terms and not evaluated on their influence on the effectiveness of implementation strategies. Future research should therefore examine if developing tailored implementation strategies in which these factors are addressed will also improve the implementation of web-based computer-tailored interventions by occupational health centers. It has been suggested that implementation rates can also be improved by involving occupational health centers in the development process of an intervention (Bartholomew et al., 2011). This makes it possible to integrate their opinion in the elaboration of the different intervention components. This is crucial because a match between the intervention and occupational health centers' preferences will make it more likely that the implementation will succeed (Bartholomew et al., 2011).

The conclusions and recommendations above should be interpreted taking into account the strengths and limitations of our study. An important strength is that this study included both adopters and non-adopters of the web-based computer-tailored intervention, which may give a better overview of the importance of the barriers and facilitators to adoption and implementation. The fact that we only interviewed directors of occupational health centers can, however, be considered as a limitation of the study. The experience of a director may not represent the experience of others in the organization. However, since 
there was much overlap in responses between participants and the fact that we reached data saturation relatively fast, we probably have identified all relevant factors. It should further also be noted that we interviewed the participants after they had made the decision to (not) implement our intervention. This could have influenced participants' answers since they may have wanted to provide a justification for their decision. The applied procedure may thus have ensured that the differences in answers between the adopting and non-adopting organizations are somewhat enlarged. Finally, as in all qualitative research, this study is limited regarding its generalizability. It has, however, been suggested that underlying beliefs are better captured through qualitative studies (Rashidian, Eccles, \& Russell, 2008).

\section{Conclusions}

To conclude, this study provides an in-depth insight into the barriers and facilitators regarding the adoption and in particular the implementation of web-based computertailored interventions by Dutch occupational health centers. Most barriers and facilitators were related to the personal beliefs of the implementer and the characteristics of the intervention. Since directors of occupational health centers can have a totally different perception of the same intervention, it is recommended to tailor implementation strategies to the unique beliefs of the person who has the responsibility for the adoption and implementation of the intervention. These implementation strategies should in particular focus on the perceived advantages of web-based computer-tailored interventions, the attitudinal and self-efficacy beliefs of the implementer, and the potential to increase occupational health centers' profits and competitiveness. 


\section{CHAPTER 8}

General discussion 
The main aim of this dissertation was to examine the appreciation, use, and efficacy of a video and text version of a web-based computer-tailored obesity prevention intervention among lower and higher educated Dutch adults with a healthy weight or limited overweight. It was hypothesized that the video version would result in better effects among people with a low educational level in particular.

The first part of this dissertation included two studies that provided insight into the psychosocial determinants of eating in moderation and the potential usefulness of clusters of dietary patterns as target for weight management interventions. The second part of this dissertation concerned the studies related to the actual intervention study. In this part insight was given into the development process as well as the efficacy and process evaluation of the video and text version of the web-based computer-tailored intervention that aimed to achieve small changes in dietary intake and physical activity in order to prevent obesity (i.e. achieve weight maintenance or modest weight loss) among Dutch adults.

This chapter will first provide an overview and reflection on the main findings of the different studies presented in this dissertation, followed by an overview of the methodological considerations. Subsequently an overview will be provided of recommendations for practice and future research. The chapter ends with a general conclusion.

\subsection{Main findings - Part 1}

The aim of the first study in part one of this dissertation was to identify the determinants of eating in moderation and to examine the possible moderating role of awareness of one's own risk behavior. The findings of this study demonstrate that half of the participants were unaware of whether or not they eat in moderation: many participants incorrectly thought that they generally ate in moderation. The average daily energy intake was significantly higher among these unaware participants. Moreover, only among the aware participants eating in moderation was significantly predicted by the psychosocial ICM factors attitude, subjective norm, social support, modelling, and intention. In line with prior research into complex behaviors (Bogers et al., 2004; Brug, Conner, et al., 2005; Greene et al., 1999; Lechner et al., 2006; Ronda et al., 2001), this study thus showed that motivational factors only predict a complex behavior (in this case eating in moderation) when people are aware of their behavior. This implies that when people do not know that their behavior is unhealthy, they will not perceive a need to change and accordingly will have no motivation to change their behavior. This result has also been found in a recent study that has shown that only a correct perception of one's BMI significantly predicts the intention to prevent weight gain (van Genugten, van Empelen, \& Oenema, 2012). Hence, our study again confirms that weight management interventions should first aim to achieve an adequate awareness of one's own risk behavior. It should further be noted that eating in moderation consists of multiple sub-behaviors (e.g. eating small portions and frequency of eating) and 
therefore cannot be regarded as one straightforward behavior such as smoking. Most sociocognitive theories, including the TPB (Ajzen, 1991) and ICM (de Vries et al., 2003), are expected to be most powerful in explaining one specific univocal behavior and it remains to be a question whether these theories can also be used for explaining a composite behavior such as eating in moderation. Our findings indicate that the ICM seems feasible for this, as the explained variances of $38 \%$ (model with psychosocial factors) and $62 \%$ (model with past behavior) are in line with studies that aimed to explain only one specific univocal behavior (Abraham \& Sheeran, 2003; Ajzen, 2011; Plotnikoff, Costigan, Karunamuni, \& Lubans, 2013).

The aim of the second study in part one was to examine if it would be more efficient to target weight management interventions at clusters of dietary patterns. For this purpose, three criteria were considered to be of importance: (1) distinctive clusters of dietary patterns can be derived, (2) cluster membership is stable over time, and (3) demographic factors can predict cluster membership and cluster transition (Correa Leite et al., 2003; Devlin et al., 2012; Grieger et al., 2012; Gubbels et al., 2013; Northstone et al., 2013; Quatromoni et al., 2002). This study has demonstrated that the first condition was met: comparable and distinctive clusters of dietary patterns were derived over time (i.e. a healthy, moderately healthy, and unhealthy dietary pattern). However, the second condition was not fully met since cluster membership was not fully stable within a 6-month follow-up period. Although not all participants changed from cluster of dietary pattern, still a large proportion of about one-third of the participants did change from dietary pattern. This implies that it may not be optimal to target interventions at a person's identified dietary pattern since it is possible that this person will have a different dietary pattern in a later stadium, which accordingly would result in delivering a misfitted intervention. In addition, the third condition was not met fully either: only few demographic factors were significantly associated with cluster membership and cluster transition. This implies that it may be difficult to target interventions at specific high-risk groups with unhealthy dietary patterns based on specific demographic factors. In conclusion, because two of the three preconditions were not met, the main conclusion of this exploratory study is that it may not be efficient to target weight management interventions at clusters of dietary patterns. It seems better to indicate via which separate food products people can achieve their behavioral and weight goal instead of addressing their whole dietary pattern. By targeting interventions at separate food products, it may still be possible to achieve improvements on other food products as well. For example, a successful change in one food product (e.g. candy bars) can stimulate a person's self-efficacy to make a change in another food product (e.g. chocolate) (Hill, 2009).

\subsection{Main findings - Part 2}

The second part of this dissertation consisted of four studies. The first study described the development process of the video and text version of the web-based computer-tailored 
obesity prevention intervention, whereas the other three studies concerned the effect and process evaluation.

\section{Efficacy}

The efficacy evaluation of the web-based computer-tailored obesity prevention intervention was presented in chapter 5. At 6-month follow-up, the video version had resulted in lower BMI and lower average daily intake of calories from energy-dense food products, while the text version only had an effect on the latter outcome (compared to a no-treatment control group). No significant effects on physical activity were found. In addition, the intervention effects were not moderated by educational level: the video and text version were equally effective for lower and higher educated participants. In conclusion, these results imply that the video version is the most effective version of the web-based computer-tailored intervention, regardless of people's educational level.

Positively, our findings contradict many previous intervention studies that have concluded that web-based computer-tailored weight management interventions generally achieve no or only small effects on both short- and long-term (Bennett \& Glasgow, 2009; Broekhuizen et al., 2012; Enwald \& Huotari, 2010; Friederichs, Bolman, Oenema, Guyaux, \& Lechner, 2014; Neville et al., 2009a, 2009b; Spittaels \& de Bourdeaudhuij, 2006; van Stralen et al., 2011; Vandelanotte et al., 2007; Wangberg et al., 2008). At 6-month followup, the video version had resulted in a significant effect on both BMI and average daily energy intake. The effect sizes of .10 for BMI and .40 for energy intake indicate that the effects are also of clinical relevance (Cohen, 2013). The small effect size on BMI will, for example, have a large public health impact when large numbers of people are reached, resulting in a significant reduction of many health problems, an improved quality of life, and cost savings (Finkelstein et al., 2012; Hill, 2009; Stevens et al., 2012; Verkleij et al., 2012). In addition, since effects on BMI are generated by changes in energy intake and energy expenditure, the potential impact of the intervention can be increased even further when it will be able to achieve a significant effect on physical activity as well.

The finding that both the video and text version did not achieve a significant effect on physical activity is in line with several reviews that have concluded that the majority of web-based computer-tailored interventions rarely achieve a significant effect on this behavior (Broekhuizen et al., 2012; Enwald \& Huotari, 2010; Friederichs et al., 2014; Neville et al., 2009b; van Stralen et al., 2011; Vandelanotte et al., 2007). A potential explanation for this could be the difficulties and problems that are common with web-based self-reported measurements of physical activity (Matthews \& Welk, 2002; Schwarz, 1999). People generally overestimate their level of activity, which results in unrealistically high physical activity scores (Shephard, 2003). This has also happened in our study as threequarters of the sample met the Dutch physical activity standard. Hence, many participants did not receive the advice to increase their physical activity level and consequently decided to make a change in their dietary intake. This makes it very unlikely and difficult to find 
improvements on physical activity. The use of a better self-report measure or an objective measurement for physical activity, such as activity monitors or GPS, would probably have prevented this problem (Reilly et al., 2008). In addition, it is also possible that no effects on physical activity were found because the intervention did not address environmental factors. Research has, for example, shown that factors within both the social (e.g. income and culture) and physical environment (e.g. (un)availability of healthy foods and sports facilities) have an important and proximal influence on people's physical activity level (Kremers et al., 2006; Wendel-Vos, Droomers, Kremers, Brug, \& van Lenthe, 2007). Yet, previous intervention studies that have targeted environmental factors have demonstrated mixed effects and this will therefore not automatically result in better intervention outcomes (Peels, Bolman, et al., 2013; Prins, Brug, van Empelen, \& Oenema, 2012; Springvloet, Lechner, de Vries, \& Oenema, 2015; van Stralen, de Vries, Bolman, Mudde, \& Lechner, 2010).

As mentioned before, we have not found support for the hypothesis that the video version would be in particular more effective for participants with a low educational level. Yet, the fact that the intervention was equally effective for both lower and higher educated participants is positive since many previous weight management interventions have only found to be effective for people with a high educational level (Magnée et al., 2013). Hence, the video version is a promising intervention that has the potential to contribute to the prevention of obesity in a general population, regardless of people's educational level. The finding that a video version is not necessarily more effective for persons with a low educational level is further in line with a recent comparable study that could not find differential effects for level of education as well, but that has demonstrated that video computer-tailoring is effective in achieving smoking cessation (Stanczyk, Bolman, et al., 2014). The absence of educational differences can further be explained by the fact that there were also no significant differences in appreciation of the intervention between lower and higher educated participants. In addition, people with a low educational level did also not prefer a video delivery format above a text format. Based on the CPM (McGuire, 1981), it can therefore be reasoned why there were no differences in intervention effects per educational level: the channel (i.e. delivery format) was not in particular more appropriate for the user (i.e. people with a low educational level) and therefore no differences in outputs (i.e. intervention outcomes) per educational level were found.

Finally, it should be noted that although this dissertation has only presented the results of the efficacy evaluation at 6-month follow-up, we also collected data at 12-month follow-up. This data can provide a better indication about whether or not the intervention is able to maintain the effects on the long-term. Preliminary analyses of this data show promising results. At 12-month follow-up, both the video and text version have resulted in a significantly lower BMI compared to the control condition. However, with regards to the secondary outcomes, both the video and text version have not achieved significant effects on average daily energy intake and physical activity. In addition, at 12-month follow-up 
again no interaction effects of educational level were found. In conclusion, these findings suggest that both the video and text version have the potential to contribute to the prevention of obesity at long-term, regardless of participants' educational level. Nevertheless, based on the better short-term effects of the video version and its higher effect sizes, it can be argued that the video version should be preferred above the text version.

\section{Appreciation}

The study presented in chapter 5 also included an evaluation of the appreciation of the webbased computer-tailored obesity prevention intervention. This evaluation firstly showed that there were no differences in appreciation between the video and text version per educational level. In contrast to the hypothesis, lower educated participants had thus not evaluated the video version better than the text version. Yet, three significant differences in appreciation were found between the video and text version regardless of participants' educational level: participants felt more related to the video version, evaluated it as more useful, and gave a higher grade to it. A potential explanation for this better evaluation may be the innovative character of the video version. Although the provision of tailored feedback via the Internet is considered to be advanced, the use of tailored video messages may be even more innovative. Hence, the video version may fit better with recent technical developments and users' expectations regarding new products (Lustria et al., 2009; Rogers, 2010). In addition, the better evaluation of the video version on feelings of relatedness can possibly also explain the higher effect sizes of this intervention. The SDT (Deci \& Ryan, 2011), for example, assumes that relatedness is an important basic need (in addition to competence and autonomy), which when satisfied can enhance persons' intrinsic motivation to change. Because the video version scored higher on feelings of relatedness, participants allocated to the video condition may have had a higher intrinsic motivation to achieve their goals (Deci \& Ryan, 2011; Ryan et al., 2008). This explanation is in line with the ELM (Petty \& Cacioppo, 1986b), which assumes that a better appreciation will stimulate central route processing. Information that is processed via this route results in stronger attitudes and a more long-lasting persuasive effect on the behavior of the receiver compared to information that is processed via the peripheral route (which is common among unmotivated persons) (Braverman, 2008; Petty \& Cacioppo, 1986b).

\section{Intervention use}

The study presented in chapter 6 provides detailed insight into the use of the video and text version as well as the influence of intervention use on the intervention outcomes. This study has demonstrated that the video version was not used more frequently than the text version, regardless of participants' level of education. Also contradicting our hypotheses, we found that more frequent intervention use did not lead to better intervention outcomes. Finally, the study demonstrated that the intervention was used more frequently when there was a match 
between the intervention delivery format and participants' preferred intervention delivery format. For example, participants who were allocated to the text condition and also preferred to receive intervention content via this delivery format (i.e. text only) had used the intervention significantly more often compared to those with a mismatch. The intervention was further also more likely to be used among participants who felt involved and supported by the intervention (i.e. feelings of relatedness).

In contrast to the expectations, the better evaluation of the video version had not resulted in more frequent intervention use. This result should, however, be interpreted in light of the fact that the use and appreciation of an intervention are also dependent on many other factors on which no significant differences could be found, such as the understandability and interestingness of intervention content (McGuire, 1981). Possibly, when the video version would have excelled on these factors as well, it would have been used more frequently.

Overall, use of both the video and text version was low and declined rapidly per session. For example, the first two intervention sessions were completed by only half of the participants and only $10.9 \%$ of the study population completed all six sessions of the intervention. Although previous studies have reported comparable figures (Bennett \& Glasgow, 2009; Spittaels, de Bourdeaudhuij, \& Vandelanotte, 2007; J. Wang et al., 2012; Wangberg et al., 2008), (repeated) intervention use was lower as expected since an explicit aim of the video version was to increase intervention use and it was pre-tested extensively. A possible explanation for the high drop-out rate from the intervention sessions may be the self-regulatory approach of the intervention: previous research has shown that there may be a lack of interest in behavior change counselling sessions (Verheijden et al., 2008; Verheijden et al., 2007). People are primarily interested in a simple comparison of their behavior against the relevant guidelines (Verheijden et al., 2008; Verheijden et al., 2007). Hence, participants may have not been interested in the last intervention sessions and accordingly had no motivation to revisit the intervention. The steep decline in repeated intervention use can possibly also be explained by the fact that the intervention required a high level of active involvement (e.g. making plans and answering a lot of questions), while participants maybe expected to receive only simple solutions. This intensive approach could have been too demanding for the participants, resulting in premature leaves (Brouwer et al., 2009; Prochaska, 2008; Schulz et al., 2012). The content of the intervention can, however, also explain the low repeated intervention use in a different way: the most crucial information for achieving a successful behavior change was included in the first two sessions. It is possible that participants were able to change their behavior successfully with the information that they received during these two sessions and consequently felt no need to visit the remaining intervention sessions. On the contrary, failure to improve may have caused that participants dropped their motivation to change and therefore stopped using the intervention. 
In line with the low (repeated) intervention use and lack of effective strategies to increase this, it seems better to limit the number of sessions of future web-based computertailored interventions and include the most important information at an early stage. This suggestion is strengthened by our finding that more intervention use was not associated with better intervention outcomes. However, there is also evidence that more intervention use does lead to better health outcomes and that people need to be exposed to educational content multiple times before effects can be expected (An et al., 2006; Brug et al., 1998; Couper et al., 2010; Dijkstra et al., 1998; Wantland et al., 2004). Hence, future interventions may still benefit from more research into strategies that can promote revisits. Our study presented in chapter 6 shows that intervention use can, for example, be stimulated by matching the delivery format of intervention content with participants' preferred intervention delivery format. In contrast to a recent study (Vandelanotte et al., 2012), our intervention was used more frequently among participants who were allocated to an intervention condition that matched their preferred intervention delivery format. However, since this match was not associated with better intervention outcomes, first more research is needed before clear conclusions can be drawn about the added value of including a preference-based delivery format tailoring option in future interventions.

\section{Recruitment}

The last study presented in part two of this dissertation provided insight into the barriers and facilitators to the implementation of a web-based computer-tailored obesity prevention intervention by directors of Dutch occupational health centers. It has been suggested that occupational health centers can implement interventions in an easy way among large numbers of people via, for example, health screenings (Hulshof et al., 1999). However, this assumed high potential implementation strategy turned out to be unsuccessful in our RCT and yielded only about one hundred participants. The findings from the qualitative study presented in chapter 7 offer a potential explanation for this failure: directors of occupational health centers can have a totally different perception of the same intervention. The directors of the adopting and implementing occupational health centers had a much more positive attitude and a more positive perception of the characteristics of the intervention than the directors of the non-adopting organizations. Hence, the use of one standard implementation strategy, as we did, will probably not be suitable since each occupational health center will take different factors into account in determining whether or not an intervention will be implemented. In line with previous research, it seems better to tailor future implementation strategies to the unique perceptions of the person who has the responsibility for the implementation of the intervention (Baker et al., 2001; Grol \& Grimshaw, 2003). Based on our findings, it can be concluded that these strategies should be tailored to the relative advantages of the intervention, occupational directors' attitude and self-efficacy, and the potential to increase occupational health centers' profits and competitiveness. In addition, according to the Intervention Mapping protocol the implementation may also be facilitated 
by involving occupational health centers in the development of an intervention (Bartholomew et al., 2011). By including the preferences and needs of the future implementers, the chance will be high that an intervention will be implemented adequately (Bartholomew et al., 2011).

However, since the implementation of our intervention by occupational health centers can be considered a failure it remains questionable whether or not collaboration with these organizations is an effective strategy for implementing web-based computertailored obesity prevention interventions. Other strategies may be more feasible for reaching and retaining large numbers of people, such as collaborating with practice nurses or mass media advertising (Smit, Hoving, Cox, \& de Vries, 2012).

\subsection{Methodological considerations}

The results of the different studies presented in this dissertation have to be interpreted in the light of numerous methodological considerations that may have affected internal and external validity. This paragraph will provide an overview of the most important methodological concerns regarding the study designs, the intervention, recruitment and response, the measurements, and the statistical analyses.

\section{Study designs}

An important strength of the studies described in the first part of this dissertation (i.e. chapter 2 and 3) is that they had a longitudinal design with a 6-month follow-up measurement. Although a longer follow-up period would have been preferred, this ensures that conclusions can be drawn about causal relationships in contrast to much cross-sectional research (Lindell \& Whitney, 2001). Moreover, a strength of the quantitative studies presented in the second part of this dissertation is the fact that they were performed with a RCT, which is considered to be the gold standard when evaluating the effects of an intervention.

Nevertheless, the RCT was also characterized by threats to the external validity. The two versions of the intervention were, for example, only compared to the control condition because the relatively small sample size prohibited a direct comparison between the two versions of the intervention. A direct comparison between the video and text version will provide a better insight into which version of the intervention should be implemented on large scale.

\section{Intervention}

The development process of the web-based computer-tailored obesity prevention intervention is an important strength. The intervention was developed in a systematic manner using the Intervention Mapping protocol and members from the target population were given the possibility to provide input during the development process (Bartholomew et al., 2011). Previous research has shown that such a well-planned and theory- and 
evidence-based development process increases the likelihood that an intervention will be effective (Bartholomew et al., 2011; Webb, Joseph, Yardley, \& Michie, 2010). Another strength is the theoretical framework that underpinned the intervention. The six sessions of the intervention were based on the consecutive behavior change steps distinguished in selfregulation theories (Leventhal et al., 1998; Zimmerman, 2000), including raising awareness, setting goals, making plans, and monitoring progress toward achieving goals. Previous research has shown that these concepts are important to successfully achieve weight management goals (Brug, Oenema, \& Ferreira, 2005; Chambers \& Swanson, 2012; de Ridder \& de Wit, 2006; Schnoll \& Zimmerman, 2001).

\section{Recruitment and response}

The high study drop-out is an important limitation of the studies related to the RCT as this lowered the power of the statistical analyses (Lenth, 2001). However, performing analyses with a very large study sample has the disadvantage that an effect of little scientific importance can be statistically detectable (Lenth, 2001). In addition, the low use of the intervention makes it difficult to draw firm conclusions about the added value of the last intervention sessions since only a small minority of the participants had completed three or more sessions. When intervention use would have been higher, the (video version of the) intervention, for example, may have been more effective since the last sessions consisted of new innovative videos (i.e. the narratives).

Another limitation is the fact that the process evaluation questionnaire was completed by only a small minority of the participants. Consequently, many participants' opinions were not reflected in the process evaluation scores. Since it was not possible to assess the reasons for drop-out, it is unclear if the inclusion of drop-outs would have led to lower or higher scores. Participants may, for example, have dropped out because they had achieved their goal or because they did not enjoy the intervention. The latter may result in a more negative evaluation, while drop-out because of success may result in a better evaluation of the intervention.

\section{Measurements}

The measurements used in the studies presented in this dissertation are also subject to limitations. All independent and dependent variables were, for example, assessed via selfreported questionnaires. Self-reported data are more likely to be inaccurate than objectively assessed variables (Nawaz, Chan, Abdulrahman, Larson, \& Katz, 2001). In particular the health outcomes (e.g. BMI and physical activity) would benefit from objective measurements (Nawaz et al., 2001; Reilly et al., 2008). Nevertheless, the conclusions of our studies are still considered to be valid. A previous study has, for example, shown that there is a high agreement between self-reported and objectively measured BMI (Dahl et al., 2010). This study has further concluded that self-reported BMI does not affect results when used as continuous variable in a longitudinal study (Dahl et al., 2010). In addition, the fact 
that participants' BMI was assessed via both online questionnaires and telephone has possibly also biased the results. It is better to assess a variable in the same manner among all participants in order to prevent measurement errors (Bland \& Altman, 2003). However, not performing an additional telephone measurement would have resulted in less data, which in turn could have prohibited statistical analyses because of a too low power.

The measurement method of intervention use can be seen as another potential limitation since it was assessed by the number of completed sessions. Although previous research has suggested that there is a high correlation between number of completed sessions and time spend at the intervention (Wangberg et al., 2008), this does not give any information about the exposure to and engagement with the intervention content. Other measures such as use of specific pages and amount of information read may, for example, give a better indication of actual intervention use (Brouwer et al., 2010; Danaher \& Seeley, 2009). Finally, appreciation of the video and text version was measured about three months after participants had last used the intervention. This long time span was chosen to prevent overload and consequently study drop-out, but may have caused that participants' could not remember the intervention in sufficient detail, which possibly has resulted in biased answers.

\section{Statistical analyses}

All statistical analyses have been performed in line with the most recent insights. However, there is ongoing debate about which statistical approaches are most suitable. Scholars, for example, differ in opinion about how interaction analyses (chapter 2) and cluster analyses (chapter 3) should be performed (Hayes, 2013; Newby \& Tucker, 2004). Moreover, an important challenge when evaluating interventions is deciding how to deal with missing values. There are many imputation techniques and each technique has strengths but also limitations, which accordingly can result in biased estimates (Donders, Van der Heijden, Stijnen, \& Moons, 2006; Musil, Warner, Yobas, \& Jones, 2002). Because it is unclear which imputation technique is most suitable, we have performed the effect analyses for the RCT with a completers only dataset as well as a dataset in which missing values were replaced using multiple imputation. An important strength is that similar results were found, regardless with which dataset the analyses were performed. It should, however, be noted that multiple imputation is not the perfect solution to deal with missing values (Blankers, Koeter, \& Schippers, 2010; White, Royston, \& Wood, 2011). There is, for example, no consensus about the required number of imputed datasets and the maximum percentage of missing values. In addition, variables that are measured multiple times are easier to replace than a variable that is only measured once such as a demographic characteristic. 


\subsection{Recommendations}

Based on the studies presented in this dissertation, the following section will provide recommendations for practice and future research.

\section{Recommendations for practice}

- The most important recommendation of this dissertation is based on the studies related to the RCT. These studies have demonstrated that the video and text version of the web-based computer-tailored intervention have the potential to decrease the BMI of both lower and higher educated adults. Since the video version had better short-term effects, higher effect sizes, and was appreciated better than the text version, the video version seems most suitable for implementation in practice.

- The video version can further be optimized by using a better measurement method for physical activity. Objective physical activity measurements such as by using GPS data can, for example, prevent the encountered problems with the assessment of physical activity in the RCT and intervention (Reilly et al., 2008). This will possibly increase the likelihood that the intervention will achieve a significant effect on this behavior. When objective measurements are not possible because of, for example, budgetary constraints, it is recommended to take people's misperception into account in tailoring algorithms by including a correction factor (Schulz, Kremers, et al., 2014).

- Based on the low and rapid decline in intervention use, it is recommended to include the most important educational content needed for achieving a successful behavior change in the first sessions. This strategy was also applied in our web-based computertailored obesity prevention intervention. The findings of the RCT imply that this strategy is effective in achieving significant and clinically relevant effects, even when only a small number of people complete all intervention sessions.

- Intervention use was higher when there was a match between the intervention delivery format and participants' preference for delivery format. Hence, to improve the use of future web-based computer-tailored interventions, it can be considered to include a delivery format preference-based tailoring option which gives participants the choice via which delivery format they want to receive intervention content. However, since a preference matched delivery format was not associated with better intervention effects, first more research is needed to determine whether or not it is cost-effective to include this option in future interventions.

- The moderating role of awareness concerning eating in moderation indicates that it is essential to firstly focus interventions on people's (un)awareness of their risk behavior. Accordingly, interventions to promote eating in moderation should first aim to create an adequate awareness level of one's own dietary behavior before targeting motivational factors. This can, for example, be done via web-based computer-tailored interventions by giving tailored feedback about how a person's behavior compares to the standards and to their peers. 
- Our findings demonstrate that dietary patterns can change over time and that it is not predictable which persons have a certain dietary pattern. This implies that dietary patterns may not be the best target for weight management interventions. Accordingly, it is recommended to target interventions at the intake of separate food products by indicating via which food products small daily energy reductions of 100 or 200 kilocalories can be made.

\section{Recommendations for future research}

- Although the video version of the web-based computer-tailored obesity prevention intervention seems to be promising, future research is needed in order to optimize this intervention and improve its effects, appreciation, and use.

- It is further recommended to examine how the intervention can be optimized among people with a low educational level. For this purpose, insight is needed into which determinants and behavior change techniques (BCT's) are most effective for these people (Abraham \& Michie, 2008; Magnée et al., 2013; Michie, Fixsen, Grimshaw, \& Eccles, 2009). The use of edutainment (i.e. combination of soap opera episodes and interactive learning modules) is, for example, considered to be a promising BCT for low health literacy patients (Jibaja-Weiss et al., 2011). Although our intervention also consisted of a type of edutainment (i.e. the narratives), only few participants received these narratives because they were included in the last intervention sessions. Future research should examine if inclusion of these narratives starting directly from the first session will result in better effects.

- Since the web-based computer-tailored intervention was able to significantly reduce the BMI of participants with a healthy weight and limited overweight (BMI lower than 30), it may be interesting to examine if the intervention can achieve a positive impact on the BMI of obese people as well. Hence, it is recommended to examine if the video and text version are also effective for people with a BMI of 30 or more.

- Intervention use was measured by means of the number of completed intervention sessions. As there are more and better indicators for intervention use, it is recommended to measure intervention use more extensively in future intervention studies, such as with log files (Kelders \& van Gemert-Pijnen, 2013). These files can, for example, indicate at which part of a session a lot of participants stopped using the intervention. This insight offers the possibility to provide additional persuasive triggers to the motivations of the user at these parts in order to support maintenance (Kelders \& van Gemert-Pijnen, 2013).

- Future research should aim at recruiting a larger study sample in order to increase the power of statistical analyses. The sample size of our RCT appeared to be too small for making a direct comparison between the effects of the video and text version (Lenth, 2001). Although it is possible to compare the effect sizes of the video and text version, 
this limits the possibility to draw valid conclusions about which version of the intervention works better and for whom.

- Although the efficacy of the intervention has been examined at 6- and 12-month follow-up, it is recommended to use a longer follow-up period in future intervention studies. Assessing intervention outcomes at, for example, two years follow-up will provide a better indication about whether or not the web-based computer-tailored intervention is actually able to prevent obesity on the long-term.

- In the studies related to the RCT, all missing values were replaced using multiple imputation. As outlined before, this technique may have shortcomings. Because attrition will never be solved completely, it is recommended to examine how the application of multiple imputation can be improved or if more optimal imputation techniques exist.

\subsection{General conclusion}

The studies presented in this dissertation show that both the video and text version of the web-based computer-tailored intervention have the potential to contribute to the prevention of obesity among Dutch adults with a healthy weight or limited overweight. In contrast to the main hypothesis, no differences in efficacy, appreciation, and intervention use between lower and higher educated people could be found. Overall, the video version appeared to be the most promising intervention as this version yielded the best short-term effects, the highest effects sizes, and was appreciated significantly better than the text version. Nevertheless, this dissertation also shows that the use of a video delivery format is not the solution to reduce the high drop-out rates that are common in web-based intervention studies: use of both the video and text version declined rapidly over time.

In conclusion, the video version of the web-based computer-tailored intervention is an effective intervention that has the potential to contribute to the prevention of obesity among large numbers of people, regardless of their educational level. Yet, future research is needed to further optimize the (video version of the) intervention in order to improve its effects, appreciation, and use. Research suggests that this can be done most efficiently by performing additional research into the effectiveness of BCT's and research on how to realize higher long-term use of the intervention. 


\section{VALORIZATION ADDENDUM}


The results of the studies presented in this dissertation provide knowledge with a high societal and economic value, which can be used for the development, optimization, and dissemination of web-based computer-tailored obesity prevention interventions. This valorization addendum will discuss the relevance of the study results, the target groups for which the results are of interest, the developed health products, the degree of innovativeness of these products, and the planning for valorization.

\section{Relevance}

Currently, about 2 billion people worldwide are overweight or obese, which is nearly $30 \%$ of the global population (Finucane et al., 2011; Stevens et al., 2012; WHO, 2015). Overweight and obese people have a significantly increased risk of developing a wide range of health problems, such as coronary heart disease, type 2 diabetes mellitus, various types of cancer, musculoskeletal disorders, respiratory problems, and mental disorders ( $\mathrm{Ng}$ et al., 2014; Visscher, van Bakel, \& Zantinge, 2014; WHO, 2015; Wyatt, Winters, \& Dubbert, 2006). Hence, overweight and obesity are associated with high direct medical costs. Research has, for example, shown that obese people spend $42 \%$ more on healthcare costs than people who have a healthy weight (Finkelstein, Trogdon, Cohen, \& Dietz, 2009). Overweight and obesity also have high indirect costs related to, for example, decreased productivity, restricted activity, and absenteeism (CDC, 2012; Finkelstein, Ruhm, \& Kosa, 2005; Neovius, Neovius, Kark, \& Rasmussen, 2012; Wyatt et al., 2006). Hence, successful weight gain prevention efforts have the potential to result in improved quality of life as well as substantial savings for both health care and society.

Web-based computer-tailored interventions are considered to be a cost-effective approach in the prevention of overweight and obesity as they can reach many people, have the potential to be effective, and can be distributed on a large scale for relatively low costs. These interventions aim to improve people's health status by providing tailored information and feedback via the Internet about, for example, people's BMI, dietary intake, physical activity level, and motivational beliefs. The studies in the second part of this dissertation show that in particular the (partial) delivery of intervention content via videos in a webbased computer-tailored intervention is an effective way to reduce adults' BMI and energy intake from energy-dense food products. Hence, the implementation of this intervention may contribute to the stabilization as well as the reduction of overweight and obesity prevalence rates among Dutch adults.

\section{Target groups}

The research described in this dissertation was funded by ZonMw, the Netherlands Organization for Health Research and Development. This organisation aims to fund health research and stimulates use of scientific knowledge to improve healthcare in the Netherlands. ZonMw acts as an intermediary between policy, research, and practice. 
Accordingly, the results presented in this dissertation are of interest to ZonMw and several other target groups.

First and most important, the results of this dissertation are of value for the actual target group of the intervention: Dutch adults with a healthy weight or overweight who want to prevent weight gain or achieve modest weight loss. The implementation of this intervention will offer these people a tool that can help them to manage their body weight.

Another important target group are health professionals, including among others occupational physicians, general practitioners, dieticians, and physical therapists. These people can use the results of the presented studies to optimize their own health services. Moreover, they are also the intermediaries that can help to implement the intervention on a large scale since they have a lot of personal contact with potential participants. The intervention can further also be implemented via national institutions involved in healthy eating, physical activity, and body weight, such as the Netherlands Institute for Sport and Physical Activity and the Netherlands Nutrition Centre Foundation. These organizations can inform administrators, policymakers, and professionals about the study results and the intervention.

The Dutch Ministry of Health, Welfare and Sport (Ministry of VWS) will also be interested in the results of the studies presented in this dissertation. The ambition of this ministry is to keep everyone in a healthy condition as long as possible and cure people with a disease as soon as possible. A primary aim is to achieve this goal by means of affordable and high-quality health care. The video version of the web-based computer-tailored obesity prevention intervention meets this aim as it can reach many people in an effective way for low costs.

The results of this dissertation may also be of interest for profit as well as nonprofit organizations that are active in the field of obesity prevention and health promotion via the Internet. For example, in the Netherlands, there is a growth in commercial organizations that are specialized in the development and implementation of eHealth. In addition, non-profit organizations, such as a municipal health service (GGD), may use the results as input for improving their own health services.

The Dutch Association of eHealth (NVEH) will also be interested in the study results. The objectives of the NVEH are among others improving the quality of eHealth products and offering a platform for interaction between eHealth producers. The NVEH also aims to act as a point of mutual interest in eHealth for patient organizations, public authorities, health insurance companies, health professionals, research, and education. In the Netherlands, nearly all health insurance companies are, for example, active in the field of health promotion and prevention. Several health insurance companies also actually reimburse participation in online health promotion interventions.

Finally, the results will also be of interest for the Center for Healthy Living (in Dutch: Centrum Gezond Leven). This organization is part of the National Institute for Public Health and the Environment (RIVM), which is an agency of the Ministry of VWS. 
The mission of the Center for Healthy Living is to contribute to the health of all Dutch citizens by presenting information about available and effective lifestyle interventions to health promotion professionals via their website. The center further presents information on the quality and efficiency of health promotion interventions.

\section{Products}

The main products of this dissertation are the video and text version of the web-based computer-tailored obesity prevention intervention. This dissertation shows that both the video and text version of the intervention are able to prevent obesity (i.e. maintain weight or achieve modest weight loss) among Dutch adults with a healthy weight or limited overweight, regardless of their educational level. Overall, the video version turned out to be the most promising intervention as this version yielded the best short-term effects, the largest effect sizes, and was appreciated significantly better than the text version.

The intervention consists of six sessions and each session takes about ten to fifteen minutes to complete. In the first session, participants receive general information and tailored feedback about their body weight, dietary intake, physical activity level, and sociocognitive beliefs toward improving their diet and physical activity level. The aim of this feedback is to help participants set an appropriate weight goal (maintain current weight or achieve modest weight loss) and behavior change goal (improve physical activity, dietary intake, or both). In session two, participants receive detailed feedback on the chosen behavior in order to help them with planning how to take feasible steps toward a successful behavior change. After this second session, participants can start with the desired changes according to their set goals and plans. The aim of session three till six is to help participants carry out and maintain the behavior change by evaluating their progress and providing tailored feedback about how to deal with barriers. These sessions are similar in content and can be used with at least one week between each session.

\section{Innovation}

Compared to existing interventions, in particular the video version of the intervention can be considered as innovative. Nearly all previous web-based computer-tailored interventions have used text-driven messages to deliver intervention content. The use of videos as delivery format is an innovative strategy that may fit better with people's current preferences as videos can deliver intervention content livelier and more interactively. In addition, the studies presented in this dissertation are one of the first that have examined the hypothesis that the use of videos to deliver intervention content would be suitable for persons with a low educational level in particular since these people generally have lower literacy skills and more difficulties with the translation of abstract text into concrete actions. The results of these studies provide important new evidence that the use of videos as delivery format in web-based computer-tailored interventions can achieve clinically relevant effects on BMI and energy intake, regardless of people's educational level. The 
finding that the video version of the intervention was equally effective for both lower and higher educated participants is also very relevant since many previous weight management interventions have only found to be effective for people with a high educational level (Magnée et al., 2013).

Besides the delivery format, our intervention is also innovative because of its content. For example, only few interventions have been based on the small changes approach which assumes that small and relatively easy to achieve behavior changes of approximately 100 kilocalories per day can prevent weight gain (Hill, 2009; Hill, Wyatt, \& Peters, 2012). In addition, in contrast to many static and predetermined interventions, our intervention also offers a lot freedom. Participants can, for example, decide themselves which goals they want to achieve and whether or not they want to follow certain intervention parts.

\section{Schedule \& Implementation}

Based on the promising results, large scale implementation of the video version of the webbased computer-tailored obesity prevention is recommended.

A potential way to facilitate large scale implementation of the video version is by submitting it for certification to the Center for Healthy Living. This center can certify the intervention as an evidence-based intervention and promote it as an appropriate obesity prevention intervention. To obtain this certificate, a committee of experts will assess the quality of the intervention based on several criteria, such as whether the intervention has been accurately described and documented, the empirical and theoretical foundation of the intervention, and the extent to which its effectiveness has been proven by means of appropriate studies. We are of the opinion that the intervention and the related studies meet all these criteria. After the quality assessment, the results of this procedure are published online in a database on the website of the Center for Healthy Living.

The video version of the web-based computer-tailored obesity prevention can be implemented in several ways. The preferred and presumed best way to implement the intervention among the most relevant target group is by collaborating with healthcare organizations, such as general practices, occupational health centers, lifestyle agencies, and eHealth organizations. Yet, the findings of this dissertation show that the implementation will only be successful when sufficient attention is paid to the development of appropriate implementation strategies. If these strategies will, for example, not address the barriers and facilitators that are considered to be relevant for the decision-maker, there is a high chance that the implementation will fail. Hence, to improve the implementation of the video version it is recommended to tailor implementation strategies to the unique perceptions of the person within an organization who has the responsibility for the implementation of the intervention. The implementation can also be improved by involving decision-makers during the further optimization process of the intervention. This offers the possibility to integrate their preferences in the intervention, which will increase the likelihood that the 
intervention will be implemented adequately (Bartholomew, Parcel, Kok, Gottlieb, \& Fernandez, 2011). Regardless of the chosen implementation strategy, it is also important that an organization will actively promote the intervention among potential implementers. For this purpose, Vision2Health can be consulted. This organization has the aim to take ownership of effective web-based lifestyle interventions and to offer these interventions to potential implementers.

When these recommendations are followed, the video version of the web-based computer-tailored obesity prevention intervention can have a large public health impact for relatively low costs. The only costs associated with the implementation of the intervention will be the hosting costs of the tailoring software package and the website. As these costs are not very high, there are hardly any risks associated with the large scale implementation of the intervention. Nevertheless, someone (e.g. the implementing organization) should take responsibility for bearing these costs, otherwise the intervention cannot be used in practice. 


\section{REFERENCES}


Abraham, C., \& Michie, S. (2008). A taxonomy of behavior change techniques used in interventions. Health Psychology, 27(3), 379-387.

Abraham, C., \& Sheeran, P. (2003). Acting on intentions: The role of anticipated regret. British Journal of Social Psychology, 42(4), 495-511.

Adams, J., \& White, M. (2005). Why don't stage-based activity promotion interventions work? Health Education Research, 20(2), 237-243.

Ajzen, I. (1991). The Theory of Planned Behavior. Organizational Behavior and Human Decision Processes, 50(2), 179-211.

Ajzen, I. (2011). The Theory of Planned Behaviour: reactions and reflections. Psychology \& Health, 26(9), 1113-1127.

Al Ghamdi, K., \& Moussa, N. (2012). Internet use by the public to search for health-related information. International Journal of Medical Informatics, 81(6), 363-373.

Alley, S., Jennings, C., Persaud, N., Plotnikoff, R., Horsley, M., \& Vandelanotte, C. (2014). Do personally tailored videos in a web-based physical activity intervention lead to higher attention and recall?-an eye-tracking study. Frontiers in Public Health, 2.

An, L., Perry, C., Lein, E., Klatt, C., Farley, D., Bliss, R., . . . Ahluwalia, J. (2006). Strategies for increasing adherence to an online smoking cessation intervention for college students. Nicotine \& Tobacco Research, 8(1), 7-12.

Arem, H., \& Irwin, M. (2011). A review of web-based weight loss interventions in adults. Obesity Reviews, 12(5), 236-243.

Armstrong, A., Idriss, N., \& Kim, R. (2011). Effects of video-based, online education on behavioral and knowledge outcomes in sunscreen use: a randomized controlled trial. Patient Education and Counseling, 83(2), 273-277.

Bailey, R., Gutschall, M., Mitchell, D., Miller, C., Lawrence, F., \& Smiciklas-Wright, H. (2006). Comparative strategies for using cluster analysis to assess dietary patterns. Journal of the American Dietetic Association, 106(8), 1194-1200.

Baker, R., Reddish, S., Robertson, N., Hearnshaw, H., \& Jones, B. (2001). Randomised controlled trial of tailored strategies to implement guidelines for the management of patients with depression in general practice. British Journal of General Practice, 51(470), 737-741.

Bandura, A. (1997). Self-efficacy: The exercise of control. New York: Freeman.

Bartholomew, L., Parcel, G., Kok, G., Gottlieb, N., \& Fernandez, M. (2011). Planning health promotion programs: An intervention mapping approach (3rd ed.). San Francisco: Jossey-Bass.

Beck, F., Richard, J., Nguyen-Thanh, V., Montagni, I., Parizot, I., \& Renahy, E. (2014). Use of the Internet as a health information resource among French young adults: Results from a nationally representative survey. Journal of Medical Internet Research, 16(5), 128. 
Bemelmans, W., Hoogenveen, R., Visscher, T., Verschuren, W., \& Schuit, A. (2004). Toekomstige ontwikkelingen in overgewicht - Inschatting effecten op de volksgezondheid. Bilthoven: RIVM.

Bennett, G., \& Glasgow, R. (2009). The delivery of public health interventions via the Internet: actualizing their potential. Annual Review of Public Health, 30, 273-292.

Bert, F., Giacometti, M., Gualano, M., \& Siliquini, R. (2014). Smartphones and health promotion: a review of the evidence. Journal of Medical Systems, 38(1), 1-11.

Bland, J., \& Altman, D. (2003). Applying the right statistics: analyses of measurement studies. Ultrasound in Obstetrics and Gynecology, 22(1), 85-93.

Blankers, M., Koeter, M., \& Schippers, G. (2010). Missing data approaches in eHealth research: simulation study and a tutorial for nonmathematically inclined researchers. Journal of Medical Internet Research, 12(5).

Blohm, I., \& Leimeister, J. (2013). Gamification. Business \& Information Systems Engineering, 5(4), 275-278.

Bogers, R., Brug, J., van Assema, P., \& Dagnelie, P. (2004). Explaining fruit and vegetable consumption: the Theory of Planned Behaviour and misconception of personal intake levels. Appetite, 42(2), 157-166.

Booth, A., Nowson, C., \& Matters, H. (2008). Evaluation of an interactive, Internet-based weight loss program: a pilot study. Health Education Research, 23(3), 371-381.

Braverman, J. (2008). Testimonials versus informational persuasive messages: The moderating effect of delivery mode and personal involvement. Communication Research, 35(5), 666-694.

Broekhuizen, K., Kroeze, W., van Poppel, M., Oenema, A., \& Brug, J. (2012). A systematic review of randomized controlled trials on the effectiveness of computer-tailored physical activity and dietary behavior promotion programs: an update. Annals of Behavioral Medicine, 44(2), 259-286.

Brouwer, W., Kroeze, W., Crutzen, R., de Nooijer, J., de Vries, N., Brug, J., \& Oenema, A. (2011). Which intervention characteristics are related to more exposure to internetdelivered healthy lifestyle promotion interventions? A systematic review. Journal of Medical Internet Research, 13(1).

Brouwer, W., Oenema, A., Crutzen, R., de Nooijer, J., de Vries, N., \& Brug, J. (2008). An exploration of factors related to dissemination of and exposure to internetdelivered behavior change interventions aimed at adults: a Delphi study approach. Journal of Medical Internet Research, 10(2).

Brouwer, W., Oenema, A., Crutzen, R., de Nooijer, J., de Vries, N., \& Brug, J. (2009). What makes people decide to visit and use an Internet-delivered behavior-change intervention? A qualitative study among adults. Health Education, 109(6), 460473.

Brouwer, W., Oenema, A., Raat, H., Crutzen, R., de Nooijer, J., de Vries, N., \& Brug, J. (2010). Characteristics of visitors and revisitors to an Internet-delivered computer- 
tailored lifestyle intervention implemented for use by the general public. Health Education Research, 25(4), 585-595.

Brown, J., Miller, W., \& Lawendowski, L. (1999). The self-regulation questionnaire. In L. Vande Creek, \& T. Jackson (Eds.), Innovations in clinical practice: A sourcebook. Sarasota: Professional Resource Press.

Brug, J., Campbell, M., \& van Assema, P. (1999). The application and impact of computergenerated personalized nutrition education: a review of the literature. Patient Education and Counseling, 36(2), 145-156.

Brug, J., Conner, M., Harré, N., Kremers, S., McKellar, S., \& Whitelaw, S. (2005). The Transtheoretical Model and stages of change: a critique Observations by five Commentators on the paper by Adams, J. and White, M. (2004) Why don't stagebased activity promotion interventions work? Health Education Research, 20(2), 244-258.

Brug, J., Glanz, K., van Assema, P., Kok, G., \& van Breukelen, G. (1998). The impact of computer-tailored feedback and iterative feedback on fat, fruit, and vegetable intake. Health Education \& Behavior, 25(4), 517-531.

Brug, J., Oenema, A., \& Campbell, M. (2003). Past, present, and future of computertailored nutrition education. American Journal of Clinical Nutrition, 77(4), 10281034.

Brug, J., Oenema, A., \& Ferreira, I. (2005). Theory, evidence and Intervention Mapping to improve behavior nutrition and physical activity interventions. International Journal of Behavioral Nutrition and Physical Activity, 2(1), 2.

Brug, J., Oenema, A., Kroeze, W., \& Raat, H. (2005). The Internet and nutrition education: challenges and opportunities. European Journal of Clinical Nutrition, 59, 130-139.

Brug, J., van Assema, P., Kok, G., Lenderink, T., \& Glanz, K. (1994). Self-rated dietary fat intake: association with objective assessment of fat, psychosocial factors, and intention to change. Journal of Nutrition Education, 26(5), 218-223.

Calvert, S., Rideout, V., Woolard, J., Barr, R., \& Strouse, G. (2005). Age, ethnicity, and socioeconomic patterns in early computer use: a national survey. American Behavioral Scientist, 48(5), 590-607.

Campbell, M., DeVellis, B., Strecher, V., Ammerman, A., DeVellis, R., \& Sandler, R. (1994). Improving dietary behavior: the effectiveness of tailored messages in primary care settings. American Journal of Public Health, 84(5), 783-787.

Campbell, M., Honess-Morreale, L., Farrell, D., Carbone, E., \& Brasure, M. (1999). A tailored multimedia nutrition education pilot program for low-income women receiving food assistance. Health Education Research, 14(2), 257-267.

Carey, K., Neal, D., \& Collins, S. (2004). A psychometric analysis of the self-regulation questionnaire. Addictive Behaviors, 29(2), 253-260. 
Carr, D., \& Friedman, M. (2005). Is obesity stigmatizing? Body weight, perceived discrimination, and psychological well-being in the United States. Journal of Health and Social Behavior, 46(3), 244-259.

CDC. (2001). Increasing physical activity. A report on recommendations of the task force on community preventive services. Atlanta: CDC.

CDC. (2012). What causes overweight and obesity?. Retrieved 2 April, 2014, from http://www.cdc.gov/obesity/adult/causes/

Chambers, J., \& Swanson, V. (2012). Stories of weight management: Factors associated with successful and unsuccessful weight maintenance. British Journal of Health Psychology, 17(2), 223-243.

Chaudoir, S., Dugan, A., \& Barr, C. (2013). Measuring factors affecting implementation of health innovations: a systematic review of structural, organizational, provider, patient, and innovation level measures. Implementation Science, 8(1), 22.

Church, T., Thomas, D., Tudor-Locke, C., Katzmarzyk, P., Earnest, C., Rodarte, R., . . . Bouchard, C. (2011). Trends over 5 decades in US occupation-related physical activity and their associations with obesity. PloS one, 6(5), e19657.

Cobiac, L., Vos, T., \& Barendregt, J. (2009). Cost-effectiveness of interventions to promote physical activity: a modelling study. PLoS medicine, 6(7), e1000110.

Cohen, J. (1992). A power primer. Psychological Bulletin, 112(1), 155-159.

Cohen, J. (2013). Statistical power analysis for the behavioral sciences (2nd ed.). New York: Routledge Academic.

Commision of the European Communities. (2007). A strategy for Europe on nutrition, overweight, and obesity related health issues. Brussel: European Communities.

Correa Leite, M., Nicolosi, A., Cristina, S., Hauser, W., Pugliese, P., \& Nappi, G. (2003). Dietary and nutritional patterns in an elderly rural population in Northern and Southern Italy: A cluster analysis of food consumption. European Journal of Clinical Nutrition, 57(12), 1514-1521.

Couper, M., Alexander, G., Zhang, N., Little, R., Maddy, N., Nowak, M., . . . Stopponi, M. (2010). Engagement and retention: measuring breadth and depth of participant use of an online intervention. Journal of Medical Internet Research, 12(4).

Crozier, S., Robinson, S., Borland, S., \& Inskip, H. (2006). Dietary patterns in the Southampton Women's Survey. European Journal of Clinical Nutrition, 60(12), 1391-1399.

Crutzen, R. (2009). Hard to get, hard to keep: dissemination of and exposure to Internetdelivered health behaviour change interventions aimed at adolescents. Maastricht: Maastricht University.

Cutler, D., \& Lleras-Muney, A. (2010). Understanding differences in health behaviors by education. Journal of Health Economics, 29(1), 1-28. 
Dahl, A., Hassing, L., Fransson, E., \& Pedersen, N. (2010). Agreement between selfreported and measured height, weight and body mass index in old age. A longitudinal study with 20 years of follow-up. Age and Ageing, 39(4), 445-451.

Damschroder, L., Aron, D., Keith, R., Kirsh, S., Alexander, J., \& Lowery, J. (2009). Fostering implementation of health services research findings into practice: a consolidated framework for advancing implementation science. Implement Science, 4(1), 50.

Danaher, B., Jazdzewski, S., McKay, H., \& Hudson, C. (2005). Bandwidth constraints to using video and other rich media in behavior change websites. Journal of Medical Internet Research, 7(4), e49.

Danaher, B., \& Seeley, J. (2009). Methodological issues in research on web-based behavioral interventions. Annals of Behavioral Medicine, 38(1), 28-39.

Darmon, N., \& Drewnowski, A. (2008). Does social class predict diet quality? American Journal of Clinical Nutrition, 87(5), 1107-1117.

de Nooijer, J., Oenema, A., Kloek, G., Brug, H., de Vries, H., \& de Vries, N. (2005). Bevordering van gezond gedrag via het Internet: nu en in de toekomst. Maastricht: Maastricht University.

de Ridder, D., \& de Wit, J. (2006). Self-regulation in health behavior. Chichester: John Wiley and Sons Ltd.

de Veer, A., Fleuren, M., Bekkema, N., \& Francke, A. (2011). Successful implementation of new technologies in nursing care: a questionnaire survey of nurse-users. $B M C$ Medical Informatics and Decision Making, 11(1), 67.

de Vries, H., \& Brug, J. (1999). Computer-tailored interventions motivating people to adopt health promoting behaviours: Introduction to a new approach. Patient Education and Counseling, 36(2), 99-105.

de Vries, H., Mudde, A., Leijs, I., Charlton, A., Vartiainen, E., Buijs, G., . . Kremers, S. (2003). The European Smoking Prevention Framework Approach (EFSA): an example of integral prevention. Health Education Research, 18(5), 611-626.

Deci, E., \& Ryan, R. (2010). Self-determination. New Jersey: John Wiley \& Sons, Inc.

Deci, E., \& Ryan, R. (2011). Self-determination theory. Handbook of theories of social psychology, 1, 416-433.

Devaux, M., \& Sassi, F. (2011). Social inequalities in obesity and overweight in 11 OECD countries. European Journal of Public Health, 23(3):464-469.

Devlin, U., McNulty, B., Nugent, A., \& Gibney, M. (2012). The use of cluster analysis to derive dietary patterns: methodological considerations, reproducibility, validity and the effect of energy mis-reporting. Proceedings of the Nutrition Society, 71(4), 599-609.

Dijkstra, A. (2008). The psychology of tailoring-ingredients in computer-tailored persuasion. Social and Personality Psychology Compass, 2(2), 765-784. 
Dijkstra, A., Conijn, B., \& de Vries, H. (2006). A match-mismatch test of a stage model of behaviour change in tobacco smoking. Addiction, 101(7), 1035-1043.

Dijkstra, A., de Vries, H., Roijackers, J., \& van Breukelen, G. (1998). Tailoring information to enhance quitting in smokers with low motivation to quit: three basic efficacy questions. Health Psychology, 17(6), 513.

Dipietro, L. (1994). Physical activity, body weight, and adiposity: an epidemiologic perspective. Exercise and Sport Sciences Reviews, 23, 275-303.

Dixon, J. (2010). The effect of obesity on health outcomes. Molecular and Cellular Endocrinology, 316(2), 104-108.

Donders, A., Van der Heijden, G., Stijnen, T., \& Moons, K. (2006). Review: A gentle introduction to imputation of missing values. Journal of Clinical Epidemiology, 59(10), 1087-1091.

Donkin, L., Christensen, H., Naismith, S., Neal, B., Hickie, I., \& Glozier, N. (2011). A systematic review of the impact of adherence on the effectiveness of e-therapies. Journal of Medical Internet Research, 13(3).

Droomers, M., Schrijvers, C., \& Mackenbach, J. (2001). Educational level and decreases in leisure time physical activity: predictors from the longitudinal GLOBE study. Journal of Epidemiology and Community Health, 55(8), 562-568.

Dumith, S., Hallal, P., Reis, R., \& Kohl, H. (2011). Worldwide prevalence of physical inactivity and its association with human development index in 76 countries. Preventive Medicine, 53(1), 24-28.

Durlak, J., \& Dupre, E. (2008). Implementation matters: A review of research on the influence of implementation on program outcomes and the factors affecting implementation. American Journal of Community Psychology, 41(3-4), 327-350.

Ello-Martin, J., Ledikwe, J., \& Rolls, B. (2005). The influence of food portion size and energy density on energy intake: implications for weight management. American Journal of Clinical Nutrition, 82(1), 236-241.

Enwald, H., \& Huotari, M. (2010). Preventing the obesity epidemic by second generation tailored health communication: an interdisciplinary review. Journal of Medical Internet Research, 12(2), e24.

Evers, K., Cummins, C., Prochaska, J., \& Prochaska, J. (2005). Online health behavior and disease management programs: are we ready for them? Are they ready for us? Journal of Medical Internet Research, 7(3), e27.

Eysenbach, G. (2005). The law of attrition. Journal of Medical Internet Research, 7(1), e11.

Eysenbach, G. (2011). CONSORT-EHEALTH: improving and standardizing evaluation reports of Web-based and mobile health interventions. Journal of Medical Internet Research, 13(4), e126. 
Finkelstein, E., Khavjou, O., Thompson, H., Trogdon, J., Pan, L., Sherry, B., \& Dietz, W. (2012). Obesity and severe obesity forecasts through 2030. American Journal of Preventive Medicine, 42(6), 563-570.

Finkelstein, E., Ruhm, C., \& Kosa, K. (2005). Economic causes and consequences of obesity. Annual Review of Public Health, 26, 239-257.

Finucane, M., Stevens, G., Cowan, M., Danaei, G., Lin, J., Paciorek, C., . . Bahalim, A. (2011). National, regional, and global trends in body-mass index since 1980: systematic analysis of health examination surveys and epidemiological studies with 960 country-years and 9.1 million participants. Lancet, 377(9765), 557-567.

Fleuren, M., Wiefferink, K., \& Paulussen, T. (2004). Determinants of innovation within health care organizations: Literature review and delphi study. International Journal for Quality in Health Care, 16(2), 107-123.

Floyd, A., \& Moyer, A. (2010). Effects of participant preferences in unblinded randomized controlled trials. Journal of Empirical Research on Human Research Ethics, 5(2), 81-93.

Francke, A., Smit, M., de Veer, A., \& Mistiaen, P. (2008). Factors influencing the implementation of clinical guidelines for health care professionals: a systematic meta-review. BMC Medical Informatics and Decision Making, 8(1), 38.

Frenn, M., Malin, S., Brown, R., Greer, Y., Fox, J., Greer, J., \& Smyczek, S. (2005). Changing the tide: an Internet/video exercise and low-fat diet intervention with middle-school students. Applied Nursing Research, 18(1), 13-21.

Friederichs, S., Bolman, C., Oenema, A., Guyaux, J., \& Lechner, L. (2014). Motivational interviewing in a web-based physical activity intervention with an avatar: Randomized controlled trial. Journal of Medical Internet Research, 16(2), e48.

Gerber, B., Brodsky, I., Lawless, K., Smolin, L., Arozullah, A., Smith, E., . . . Eiser, A. (2005). Implementation and evaluation of a low-literacy diabetes education computer multimedia application. Diabetes Care, 28(7), 1574-1580.

Giskes, K., Avendaňo, M., Brug, J., \& Kunst, A. (2010). A systematic review of studies on socioeconomic inequalities in dietary intakes associated with weight gain and overweight/obesity conducted among European adults. Obesity Reviews, 11(6), 413-429.

Giskes, K., Turrell, G., van Lenthe, F., Brug, J., \& Mackenbach, J. (2006). A multilevel study of socio-economic inequalities in food choice behaviour and dietary intake among the Dutch population: the GLOBE study. Public Health Nutrition, 9(1), 7583.

Glanz, K., Brug, J., \& van Assema, P. (1997). Are awareness of dietary fat intake and actual fat consumption associated? a Dutch-American comparison. European Journal of Clinical Nutrition, 51(8), 542-547.

Glasgow, R. (2007). eHealth evaluation and dissemination research. American Journal of Preventive Medicine, 32(5), 119-126. 
Glasgow, R., Nelson, C., Kearney, K., Reid, R., Ritzwoller, D., Strecher, V., . . . Wildenhaus, K. (2007). Reach, engagement, and retention in an Internet-based weight loss program in a multi-site randomized controlled trial. Journal of Medical Internet Research, 9(2), e11.

Glasgow, R., Vogt, T., \& Boles, S. (1999). Evaluating the public health impact of health promotion interventions: the RE-AIM framework. American Journal of Public Health, 89(9), 1322-1327.

Gortmaker, S., Swinburn, B., Levy, D., Carter, R., Mabry, P., Finegood, D., . . . Moodie, M. (2011). Changing the future of obesity: science, policy, and action. Lancet, 378(9793), 838-847.

Grafenauer, S., Tapsell, L., Beck, E., \& Batterham, M. (2013). Baseline dietary patterns are a significant consideration in correcting dietary exposure for weight loss. European Journal of Clinical Nutrition, 67(4), 330-336.

Green, L., \& Kreuter, M. (2005). Health promotion planning: An educational and ecological approach (4th ed.). New York: McGraw-Hill.

Greene, G., Rossi, S., Rossi, J., Velicer, W., Fava, J., \& Prochaska, J. (1999). Dietary applications of the stages of change model. Journal of the American Dietetic Association, 99(6), 673-678.

Greenhalgh, T., Robert, G., Macfarlane, F., Bate, P., \& Kyriakidou, O. (2004). Diffusion of innovations in service organizations: systematic review and recommendations. Milbank Quarterly, 82(4), 581-629.

Greenwood, D., Cade, J., Draper, A., Barrett, J., Calvert, C., \& Greenhalgh, A. (2000). Seven unique food consumption patterns identified among women in the UK Women's Cohort Study. European Journal of Clinical Nutrition, 54(4), 314-320.

Gretebeck, R., Ferraro, K., Black, D., Holland, K., \& Gretebeck, K. (2012). Longitudinal change in physical activity and disability in adults. American Journal of Health Behavior, 36(3), 385-394.

Grieger, J., Scott, J., \& Cobiac, L. (2012). Cluster analysis and food group consumption in a national sample of Australian girls. Journal of Human Nutrition and Dietetics, 25(1), 75-86.

Grimshaw, J., Eccles, M., \& Tetroe, J. (2004). Implementing clinical guidelines: current evidence and future implications. Journal of Continuing Education in the Health Professions, 24(1), 31-37.

Grol, R., \& Grimshaw, J. (2003). From best evidence to best practice: effective implementation of change in patients' care. Lancet, 362(9391), 1225-1230.

Grol, R., \& Wensing, M. (2004). What drives change? Barriers to and incentives for achieving evidence-based practice. The Medical Journal of Australia, 180(6), 57 60 .

Gubbels, J., van Assema, P., \& Kremers, S. (2013). Physical activity, sedentary behavior, and dietary patterns among children. Current Nutrition Reports, 1-8. 
Hagger, M., Chatzisarantis, N., \& Biddle, S. (2002). A meta-analytic review of the theories of reasoned action and planned behavior in physical activity: Predictive validity and the contribution of additional variables. Journal of Sport \& Exercise Psychology, 24(1), 3-32.

Haines, P., Siega-Riz, A., \& Popkin, B. (1999). The Diet Quality Index revised: a measurement instrument for populations. Journal of the American Dietetic Association, 99(6), 697-704.

Hall, K., Heymsfield, S., Kemnitz, J., Klein, S., Schoeller, D., \& Speakman, J. (2012). Energy balance and its components: implications for body weight regulation. American Journal of Clinical Nutrition, 95(4), 989-994.

Hall, K., Sacks, G., Chandramohan, D., Chow, C., Wang, Y., Gortmaker, S., \& Swinburn, B. (2011). Quantification of the effect of energy imbalance on bodyweight. Lancet, 378(9793), 826-837.

Hallal, P., Andersen, L., Bull, F., Guthold, R., Haskell, W., \& Ekelund, U. (2012). Global physical activity levels: surveillance progress, pitfalls, and prospects. Lancet, 380(9838), 247-257.

Hansen, A., Grønbæk, M., Helge, J., Severin, M., Curtis, T., \& Tolstrup, J. (2012). Effect of a web-based intervention to promote physical activity and improve health among physically inactive adults: a population-based randomized controlled trial. Journal of Medical Internet Research, 14(5), e145.

Hawkins, R., Kreuter, M., Resnicow, K., Fishbein, M., \& Dijkstra, A. (2008). Understanding tailoring in communicating about health. Health Education Research, 23(3), 454-466.

Hayes, A. (2013). Introduction to mediation, moderation, and conditional process analysis: A regression-based approach. New York: Guilford Press.

Hearty, A., \& Gibney, M. (2009). Comparison of cluster and principal component analysis techniques to derive dietary patterns in Irish adults. British Journal of Nutrition, 101(4), 598-608.

Hearty, A., \& Gibney, M. (2013). Dietary patterns in Irish adolescents: a comparison of cluster and principal component analyses. Public Health Nutrition, 16(5), 848-857.

Helfrich, C., Weiner, B., McKinney, M., \& Minasian, L. (2007). Determinants of implementation effectiveness: Adapting a framework for complex innovations. Medical Care Research and Review, 64(3), 279-303.

Helmink, J., Kremers, S., van Boekel, L., van Brussel-Visser, F., \& de Vries, N. (2012). Factors determining the motivation of primary health care professionals to implement and continue the 'Beweegkuur' lifestyle intervention programme. Journal of Evaluation in Clinical Practice, 18(3), 682-688.

Hill, J. (2006). Understanding and addressing the epidemic of obesity: an energy balance perspective. Endocrine reviews, 27(7), 750-761. 
Hill, J. (2009). Can a small-changes approach help address the obesity epidemic? A report of the Joint Task Force of the American Society for Nutrition, Institute of Food Technologists, and International Food Information Council. American Journal of Clinical Nutrition, 89(2), 477-484.

Hill, J., Wyatt, H., \& Melanson, E. (2000). Genetic and environmental contributions to obesity. Medical Clinics of North America, 84(2), 333-346.

Hill, J., Wyatt, H., \& Peters, J. (2012). Energy balance and obesity. Circulation, 126(1), 126-132.

Hill, J., Wyatt, H., Reed, G., \& Peters, J. (2003). Obesity and the environment: where do we go from here? Science, 299(5608), 853-855.

Hinyard, L., \& Kreuter, M. (2007). Using narrative communication as a tool for health behavior change: A conceptual, theoretical, and empirical overview. Health Education \& Behavior, 34(5), 777-792.

Houts, P., Doak, C., Doak, L., \& Loscalzo, M. (2006). The role of pictures in improving health communication: a review of research on attention, comprehension, recall, and adherence. Patient Education and Counseling, 61(2), 173-190.

Hsieh, H., \& Shannon, S. (2005). Three approaches to qualitative content analysis. Qualitative Health Research, 15(9), 1277-1288.

Hu, F., Rimm, E., Smith-Warner, S., Feskanich, D., Stampfer, M., Ascherio, A., . . . Willett, W. (1999). Reproducibility and validity of dietary patterns assessed with a food-frequency questionnaire. American Journal of Clinical Nutrition, 69(2), 243249.

Hughes, M., Patrick, D., Hannon, P., Harris, J., \& Ghosh, D. (2011). Understanding the decision-making process for health promotion programming at small to midsized businesses. Health Promotion Practice, 12(4), 512-521.

Hulshof, C., Verbeek, J., van Dijk, F., Van der Weide, W., \& Braam, I. (1999). Evaluation research in occupational health services: general principles and a systematic review of empirical studies. Occupational and Environmental Medicine, 56(6), 361-377.

Internet World Stats. (2013). Internet usage in Europe: Internet user statistics and population for 53 European countries and regions. Retrieved 20 October, 2013, from http://www.internetworldstats.com/stats4.htm

Internet World Stats. (2014). Internet users in Europe. Retrieved 30 April 2014, from http://www.internetworldstats.com/stats4.htm

Jacobson, T., Thomas, D., Morton, F., Offutt, G., Shevlin, J., \& Ray, S. (1999). Use of a low-literacy patient education tool to enhance pneumococcal vaccination rates: a randomized controlled trial. Journal of the American Medical Association, 282(7), 646-650.

Janz, N., \& Becker, M. (1984). The health belief model: A decade later. Health Education \& Behavior, 11(1), 1-47. 
Jibaja-Weiss, M., Volk, R., Granchi, T., Neff, N., Robinson, E., Spann, S., . . . Beck, J. (2011). Entertainment education for breast cancer surgery decisions: a randomized trial among patients with low health literacy. Patient Education and Counseling, 84(1), 41-48.

Kandula, N., Nsiah-Kumi, P., Makoul, G., Sager, J., Zei, C., Glass, S., . . . Baker, D. (2009). The relationship between health literacy and knowledge improvement after a multimedia type 2 diabetes education program. Patient Education and Counseling, 75(3), 321-327.

Kant, A. (2004). Dietary patterns and health outcomes. Journal of the American Dietetic Association, 104(4), 615-635.

Kathleen, E. (1997). Video-mediated communication. New Jersey: Lawrence Erlbaum Associates.

Kayman, S., Bruvold, W., \& Stern, J. (1990). Maintenance and relapse after weight loss in women: behavioral aspects. American Journal of Clinical Nutrition, 52(5), 800807.

Kelders, S., \& van Gemert-Pijnen, J. (2013). Using log-data as a starting point to make eHealth more persuasive. Persuasive Technology, 99-109.

Kidwell, B., \& Jewell, R. (2008). The influence of past behavior on behavioral intent: An information-processing explanation. Psychology \& Marketing, 25(12), 1151-1166.

Kiesler, D., \& Auerbach, S. (2006). Optimal matches of patient preferences for information, decision-making and interpersonal behavior: evidence, models and interventions. Patient Education and Counseling, 61(3), 319-341.

King, M. (2000). The effects of patients' and practitioners' preferences on randomized clinical trials. Palliative Medicine, 14(6), 539-542.

King, M., Nazareth, I., Lampe, F., Bower, P., Chandler, M., Morou, M., . . Lai, R. (2005). Impact of participant and physician intervention preferences on randomized trials: a systematic review. Journal of the American Medical Association, 293(9), 10891099.

Klein, K., \& Sorra, J. (1996). The challenge of innovation implementation. Academy of Management Review, 21(4), 1055-1080.

Klem, M., Wing, R., McGuire, M., Seagle, H., \& Hill, J. (1997). A descriptive study of individuals successful at long-term maintenance of substantial weight loss. American Journal of Clinical Nutrition, 66(2), 239-246.

Kloek, G., van Lenthe, F., van Nierop, P., \& Mackenbach, J. (2004). Stages of change for fruit and vegetable consumption in deprived neighborhoods. Health Education \& Behavior, 31(2), 223-241.

Konttinen, H., Sarlio-Lähteenkorva, S., Silventoinen, K., Männistö, S., \& Haukkala, A. (2013). Socio-economic disparities in the consumption of vegetables, fruit and energy-dense foods: the role of motive priorities. Public Health Nutrition, 16(05), 873-882. 
Koppelaar, E., Knibbe, H., Miedema, H., \& Burdorf, A. (2009). Determinants of implementation of primary preventive interventions on patient handling in healthcare: a systematic review. Occupational and Environmental Medicine, 66(6), 353-360.

Kral, T., Roe, L., \& Rolls, B. (2004). Combined effects of energy density and portion size on energy intake in women. American Journal of Clinical Nutrition, 79(6), $962-$ 968.

Krawczyk, A., Lau, E., Perez, S., Delisle, V., Amsel, R., \& Rosberger, Z. (2012). How to inform: comparing written and video education interventions to increase human papillomavirus knowledge and vaccination intentions in young adults. Journal of American College Health, 60(4), 316-322.

Krebs, P., Prochaska, J., \& Rossi, J. (2010). A meta-analysis of computer-tailored interventions for health behavior change. Preventive Medicine, 51(3), 214-221.

Kremers, S., de Bruijn, G., Visscher, T., van Mechelen, W., de Vries, N., \& Brug, J. (2006). Environmental influences on energy balance-related behaviors: A dual-process view. International Journal of Behavioral Nutrition and Physical Activity, 3(1), 9.

Kreuter, M., \& Skinner, C. (2000). Tailoring: what's in a name? Health Education Research, 15(1), 1-4.

Krishnan, S., \& Sitaraman, R. (2013). Video stream quality impacts viewer behavior: inferring causality using quasi-experimental designs. IEEE/ACM Transactions on Networking, 21(6), 2001-2014.

Kroeze, W., Werkman, A., \& Brug, J. (2006). A systematic review of randomized trials on the effectiveness of computer-tailored education on physical activity and dietary behaviors. Annals of Behavioral Medicine, 31(3), 205-223.

Krouse, H. (2001). Video modelling to educate patients. Journal of Advanced Nursing, 33(6), 748-757.

Kumar, S., Nilsen, W., Abernethy, A., Atienza, A., Patrick, K., Pavel, M., . . S Spruijt-Metz, D. (2013). Mobile health technology evaluation: the mHealth evidence workshop. American Journal of Preventive Medicine, 45(2), 228-236.

Kypri, K., \& McAnally, H. (2005). Randomized controlled trial of a web-based primary care intervention for multiple health risk behaviors. Preventive Medicine, 41(3), 761-766.

Lancaster, B. (1999). Defining and interpreting suppressor effects: Advantages and limitations. In B. Thompson (Ed.), Advances in social science methodology (pp. 139-148). Stamford: JAI Press.

Landis, J., \& Koch, G. (1977). The measurement of observer agreement for categorical data. Biometrics, 159-174.

Latham, G., \& Locke, E. (1991). Self-regulation through goal setting. Organizational Behavior and Human Decision Processes, 50, 212-247. 
Lechner, L., Bolman, C., \& Van Dijke, M. (2006). Factors related to misperception of physical activity in The Netherlands and implications for health promotion programmes. Health Promotion International, 21(2), 104-112.

Lechner, L., Brug, J., \& de Vries, H. (1997). Misconceptions of fruit and vegetable consumption: differences between objective and subjective estimation of intake. Journal of Nutrition Education, 29(6), 313-320.

Lechner, L., Brug, J., de Vries, H., van Assema, P., \& Mudde, A. (1998). Stages of change for fruit, vegetable and fat intake: consequences of misconception. Health Education Research, 13(1), 1-11.

Lee, J. (2011). Effect of web-based interactive tailored health videos on users' attention, interactivity, overall evaluation, preference and engagement. Proceedings of the American Society for Information Science and Technology, 48(1), 1-3.

Lenert, L., Muñoz, R., Stoddard, J., Delucchi, K., Bansod, A., Skoczen, S., \& Pérez-Stable, E. (2003). Design and pilot evaluation of an internet smoking cessation program. Journal of the American Medical Informatics Association, 10(1), 16-20.

Lenth, R. (2001). Some practical guidelines for effective sample size determination. American Statistician, 55(3), 187-193.

Leslie, E., Marshall, A., Owen, N., \& Bauman, A. (2005). Engagement and retention of participants in a physical activity website. Preventive Medicine, 40(1), 54-59.

Leventhal, H., Leventhal, E., \& Contrada, R. (1998). Self-regulation, health, and behavior: A perceptual-cognitive approach. Psychology \& Health, 13(4), 717-733.

Li, J., Land, L., \& Ray, P. (2008). Humanitarian Technology Challenge (HTC)-electronic health records perspective. IEEE and United Nations Foundation.

Li, J., Talaei-Khoei, A., Seale, H., Ray, P., \& MacIntyre, C. (2013). Health care provider adoption of eHealth: Systematic literature review. Interactive Journal of Medical Research, 2(1), e7.

Lindell, M., \& Whitney, D. (2001). Accounting for common method variance in crosssectional research designs. Journal of Applied Psychology, 86(1), 114-121.

Lustria, M., Cortese, J., Noar, S., \& Glueckauf, R. (2009). Computer-tailored health interventions delivered over the Web: review and analysis of key components. Patient Education and Counseling, 74(2), 156-173.

Lutes, L., Winett, R., Barger, S., Wojcik, J., Herbert, W., Nickols-Richardson, S., \& Anderson, E. (2008). Small changes in nutrition and physical activity promote weight loss and maintenance: 3-month evidence from the ASPIRE randomized trial. Annals of Behavioral Medicine, 35(3), 351-357.

Ma, Y., Hua, X., Lu, L., \& Zhang, H. (2005). A generic framework of user attention model and its application in video summarization. IEEE Transactions on Multimedia, 7(5), 907-919. 
Magnée, T., Burdorf, A., Brug, J., Kremers, S., Oenema, A., van Assema, P., . . HopmanRock, M. (2013). Equity-specific effects of 26 Dutch obesity-related lifestyle interventions. American Journal of Preventive Medicine, 44(6), 61-70.

Mair, F., May, C., O'Donnell, C., Finch, T., Sullivan, F., \& Murray, E. (2012). Factors that promote or inhibit the implementation of e-health systems: an explanatory systematic review. WHO, 90(5), 357-364.

Malterud, K., \& Tonstad, S. (2009). Preventing obesity: Challenges and pitfalls for health promotion. Patient Education and Counseling, 76(2), 254-259.

Marcus, B., Nigg, C., Riebe, D., \& Forsyth, L. (2000). Interactive communication strategies: implications for population-based physical-activity promotion. American Journal of Preventive Medicine, 19(2), 121-126.

Marcus, B., Owen, N., Forsyth, L., Cavill, N., \& Fridinger, F. (1998). Physical activity interventions using mass media, print media, and information technology. American Journal of Preventive Medicine, 15(4), 362-378.

Martikainen, P., Brunner, E., \& Marmot, M. (2003). Socioeconomic differences in dietary patterns among middle-aged men and women. Social Science \& Medicine, 56(7), 1397-1410.

Matthews, C., \& Welk, G. (2002). Use of self-report instruments to assess physical activity. Physical Activity Assessments for Health-Related Research, 107-123.

Mayer, R., \& Massa, L. (2003). Three facets of visual and verbal learners: cognitive ability, cognitive style, and learning preference. Journal of Educational Psychology, 95(4), 833-846.

McCrory, M., Howarth, N., Roberts, S., \& Huang, T. (2011). Eating frequency and energy regulation in free-living adults consuming self-selected diets. Journal of Nutrition, 141(1), 148-153.

McGuire, W. (1981). Theoretical foundations of campaigns. In R. Rice, \& W. Paisley (Eds.), Public communication campaigns (pp. 41-70). Beverly Hills: Sage.

McGuire, W. (1985). Attitudes and attitude change. In G. Aronson (Ed.), Handbook of social psychology (pp. 233-246). New York: Lawrence Erlbaum Associates.

McGuire, W. (2000). Standing on the shoulders of ancients: Consumer research, persuasion, and figurative language. Journal of Consumer Research, 27(1), 109114.

McLaren, L. (2007). Socioeconomic status and obesity. Epidemiologic Reviews, 29(1), 2948.

Meade, C. (1996). Producing videotapes for cancer education: methods and examples. Oncology Nursing Forum, 23(5), 837-846.

Michels, K., \& Schulze, M. (2005). Can dietary patterns help us detect diet-disease associations? Nutrition Research Reviews, 18(02), 241-248. 
Michie, S., Abraham, C., Whittington, C., McAteer, J., \& Gupta, S. (2009). Effective techniques in healthy eating and physical activity interventions: A metaregression. Health Psychology, 28(6), 690-701.

Michie, S., Ashford, S., Sniehotta, F., Dombrowski, S., Bishop, A., \& French, D. (2011). A refined taxonomy of behaviour change techniques to help people change their physical activity and healthy eating behaviours: The CALO-RE taxonomy. Psychology \& Health, 26(11), 1479-1498.

Michie, S., Fixsen, D., Grimshaw, J., \& Eccles, M. (2009). Specifying and reporting complex behaviour change interventions: the need for a scientific method. Implementation Science, 4(40), 1-6.

Millen, B., Quatromoni, P., Copenhafer, D., Demissie, S., O'Horo, C., \& D'Agostino, R. (2001). Validation of a dietary pattern approach for evaluating nutritional risk: the Framingham Nutrition Studies. Journal of the American Dietetic Association, 101(2), 187-194.

Millen, B., Quatromoni, P., Pencina, M., Kimokoti, R., Nam, B., Cobain, S., . . . D'Agostino, R. (2005). Unique dietary patterns and chronic disease risk profiles of adult men: the Framingham nutrition studies. Journal of the American Dietetic Association, 105(11), 1723-1734.

Milligan, G., \& Cooper, M. (1987). Methodology review: Clustering methods. Applied Psychological Measurement, 11(4), 329-354.

Mo, F., Turner, M., Krewski, D., \& Mo, F. (2005). Physical inactivity and socioeconomic status in Canadian adolescents. International Journal of Adolescent Medicine and Health, 17(1), 49-56.

Moen, A., Hackl, W., Hofdijk, J., van Gemert-Pijnen, L., Ammenwerth, E., Nykänen, P., \& Hoerbst, A. (2012). eHealth in Europe: status and challenges. European Journal for Biomedical Informatics, 8(1), 2-7.

Monteiro, C., Moura, E., Conde, W., \& Popkin, B. (2004). Socioeconomic status and obesity in adult populations of developing countries: a review. WHO, 82, 940-946.

Mozaffarian, D., Hao, T., Rimm, E., Willett, W., \& Hu, F. (2011). Changes in diet and lifestyle and long-term weight gain in women and men. New England Journal of Medicine, 364(25), 2392-2404.

Murray, E., Burns, J., May, C., Finch, T., O'Donnell, C., Wallace, P., \& Mair, F. (2011). Why is it difficult to implement e-health initiatives? A qualitative study. Implementation Science, 6(6).

Musil, C., Warner, C., Yobas, P., \& Jones, S. (2002). A comparison of imputation techniques for handling missing data. Western Journal of Nursing Research, 24(7), 815-829.

Nawaz, H., Chan, W., Abdulrahman, M., Larson, D., \& Katz, D. (2001). Self-reported weight and height: Implications for obesity research. American Journal of Preventive Medicine, 20(4), 294-298. 
Neovius, K., Neovius, M., Kark, M., \& Rasmussen, F. (2012). Association between obesity status and sick-leave in Swedish men: nationwide cohort study. European Journal of Public Health, 22(1), 112-116.

Neville, L., O'Hara, B., \& Milat, A. (2009a). Computer-tailored dietary behaviour change interventions: a systematic review. Health Education Research, 24(4), 699-720.

Neville, L., O'Hara, B., \& Milat, A. (2009b). Computer-tailored physical activity behavior change interventions targeting adults: a systematic review. International Journal of Behavioral Nutrition and Physical Activity, 6(1), 30.

Newby, P., Muller, D., Hallfrisch, J., Qiao, N., Andres, R., \& Tucker, K. (2003). Dietary patterns and changes in body mass index and waist circumference in adults. American Journal of Clinical Nutrition, 77(6), 1417-1425.

Newby, P., \& Tucker, K. (2004). Empirically derived eating patterns using factor or cluster analysis: a review. Nutrition Reviews, 62(5), 177-203.

Ng, M., Fleming, T., Robinson, M., Thomson, B., Graetz, N., Margono, C., . . . Abera, S. (2014). Global, regional, and national prevalence of overweight and obesity in children and adults during 1980-2013: a systematic analysis for the Global Burden of Disease Study 2013. Lancet, 384(9945), 766-781.

NHLBI. (2012). What causes overweight and obesity?. Retrieved 16 October, 2012, from http://www.nhlbi.nih.gov/health/health-topics/topics/obe/causes.html

Northstone, K., Smith, A., Newby, P., \& Emmett, P. (2013). Longitudinal comparisons of dietary patterns derived by cluster analysis in 7- to 13-year-old children. British Journal of Nutrition, 109(11), 2050-2058.

Oenema, A., \& Brug, J. (2003). Feedback strategies to raise awareness of personal dietary intake: results of a randomized controlled trial. Preventive Medicine, 36(4), 429439.

Oenema, A., Brug, J., Dijkstra, A., de Weerdt, I., \& de Vries, H. (2008). Efficacy and use of an internet-delivered computer-tailored lifestyle intervention, targeting saturated fat intake, physical activity and smoking cessation: a randomized controlled trial. Annals of Behavioral Medicine, 35(2), 125-135.

Oenema, A., Brug, J., \& Lechner, L. (2001). Web-based tailored nutrition education: results of a randomized controlled trial. Health Education Research, 16(6), 647-660.

Paasche-Orlow, M., Parker, R., Gazmararian, J., Nielsen-Bohlman, L., \& Rudd, R. (2005). The prevalence of limited health literacy. Journal of General Internal Medicine, 20(2), 175-184.

Peels, D., Bolman, C., Golsteijn, R., de Vries, H., Mudde, A., van Stralen, M., \& Lechner, L. (2013). Long-term efficacy of a printed or a web-based tailored physical activity intervention among older adults. International Journal of Behavioral Nutrition and Physical Activity, 10, 104.

Peels, D., de Vries, H., Bolman, C., Golsteijn, R., van Stralen, M., Mudde, A., \& Lechner, L. (2013). Differences in the use and appreciation of a web-based or printed 
computer-tailored physical activity intervention for people aged over 50 years. Health Education Research, 28(4), 715-731.

Peels, D., Mudde, A., Bolman, C., Golsteijn, R., de Vries, H., \& Lechner, L. (2014). Correlates of the intention to implement a tailored physical activity intervention: Perceptions of intermediaries. International Journal of Environmental Research and Public Health, 11(2), 1885-1903.

Petty, R., \& Cacioppo, J. (1986a). Communication and persuasion: Central and peripheral routes to attitude change. New York: Springer-Verlag.

Petty, R., \& Cacioppo, J. (1986b). The Elaboration Likelihood Model of persuasion. Advances in Experimental Social Psychology, 19, 123-205.

Plotnikoff, R., Costigan, S., Karunamuni, N., \& Lubans, D. (2013). Social cognitive theories used to explain physical activity behavior in adolescents: a systematic review and meta-analysis. Preventive Medicine, 56(5), 245-253.

Pollard, J., Kirk, S., \& Cade, J. (2002). Factors affecting food choice in relation to fruit and vegetable intake: a review. Nutrition Research Reviews, 15(2), 373-388.

Portnoy, D., Scott-Sheldon, L., Johnson, B., \& Carey, M. (2008). Computer-delivered interventions for health promotion and behavioral risk reduction: a meta-analysis of 75 randomized controlled trials, 1988-2007. Preventive Medicine, 47(1), 3-16.

Prins, R., Brug, J., van Empelen, P., \& Oenema, A. (2012). Effectiveness of YouRAction, an intervention to promote adolescent physical activity using personal and environmental feedback: a cluster RCT. PloS one, 7(3), e32682.

Prochaska, J. (2008). Multiple health behavior research represents the future of preventive medicine. Preventive Medicine, 46(3), 281-285.

Prochaska, J., \& Velicer, W. (1997). The Transtheoretical Model of health behavior change. American Journal of Health Promotion, 12(1), 38-48.

Pryer, J., Nichols, R., Elliott, P., Thakrar, B., Brunner, E., \& Marmot, M. (2001). Dietary patterns among a national random sample of British adults. Journal of Epidemiology and Community Health, 55(1), 29-37.

PWC. (2012). Emerging mHealth: paths for growth. Retrieved 30 April, 2014, from http://www.pwc.com/gx/en/healthcare/mhealth/assets/pwc-emerging-mhealthfull.pdf

Quatromoni, P., Copenhafer, D., Demissie, S., D'Agostino, R., O'Horo, C., Nam, B., \& Millen, B. (2002). The internal validity of a dietary pattern analysis. The Framingham Nutrition Studies. Journal of Epidemiology and Community Health, 56(5), 381-388.

Rashidian, A., Eccles, M., \& Russell, I. (2008). Falling on stony ground? A qualitative study of implementation of clinical guidelines' prescribing recommendations in primary care. Health Policy, 85(2), 148-161. 
Reilly, J., Penpraze, V., Hislop, J., Davies, G., Grant, S., \& Paton, J. (2008). Objective measurement of physical activity and sedentary behaviour: review with new data. Archives of Disease in Childhood, 93(7), 614-619.

RIVM. (2012). Bevolkingsonderzoeken en screeningen. Retrieved 29 July, 2012, from http://www.rivm.nl/Onderwerpen/B/Bevolkingsonderzoeken_en_screeningen/Ach tergrondinformatie/Screening_de_theorie/Wie_bieden_screeningen_aan

RIVM. (2013). Dutch Food Composition Dataset (NEVO). Den Haag: Voedingscentrum.

Robroek, S., van Lenthe, F., van Empelen, P., \& Burdorf, A. (2009). Determinants of participation in worksite health promotion programmes: a systematic review. International Journal of Behavioral Nutrition and Physical Activity, 6, 26.

Rodearmel, S., Wyatt, H., Stroebele, N., Smith, S., Ogden, L., \& Hill, J. (2007). Small changes in dietary sugar and physical activity as an approach to preventing excessive weight gain: the America on the Move family study. Pediatrics, 120(4), 869-879.

Rogers, E. (2010). Diffusion of innovations. New York: Simon and Schuster.

Rogers, R., \& Prentice-Dunn, S. (1997). Protection Motivation Theory. In D. Gochman (Ed.), Handbook of health behavior research I: Personal and social determinants (pp. 113-132). New York: Plenum Press.

Rolls, B. (2009). The relationship between dietary energy density and energy intake. Physiology \& Behavior, 97(5), 609-615.

Rolls, B., Roe, L., \& Meengs, J. (2006). Reductions in portion size and energy density of foods are additive and lead to sustained decreases in energy intake. American Journal of Clinical Nutrition, 83(1), 11-17.

Rolls, B., Roe, L., \& Meengs, J. (2007). The effect of large portion sizes on energy intake is sustained for 11 days. Obesity, 15(6), 1535-1543.

Ronda, G., van Assema, P., \& Brug, J. (2001). Stages of change, psychological factors and awareness of physical activity levels in the Netherlands. Health Promotion International, 16(4), 305-314.

Roskam, A., Kunst, A., van Oyen, H., Demarest, S., Klumbiene, J., Regidor, E., . . . Mackenbach, J. (2010). Comparative appraisal of educational inequalities in overweight and obesity among adults in 19 European countries. International Journal of Epidemiology, 39(2), 392-404.

Rosnow, R., \& Rosenthal, R. (1989). Statistical procedures and the justification of knowledge in psychological science. American Psychologist, 44(10), 1276.

Ruiter, R., Kessels, L., Jansma, B., \& Brug, J. (2006). Increased attention for computertailored health communications: An event-related potential study. Health Psychology, 25(3), 300.

Ryan, R., \& Deci, E. (2000). Self-determination theory and the facilitation of intrinsic motivation, social development, and well-being. American Psychologist, 55(1), 68-78. 
Ryan, R., Patrick, H., Deci, E., \& Williams, G. (2008). Facilitating health behaviour change and its maintenance: Interventions based on Self-Determination Theory. European Health Psychologist, 10(1), 2-5.

Salomon, G. (1984). Television is "easy" and print is "tough": The differential investment of mental effort in learning as a function of perceptions and attributions. Journal of Educational Psychology, 76(4), 647.

Salomon, G., \& Gardner, H. (1986). The computer as educator: Lessons from television research. Educational Researcher, 15(1), 13-17.

Savelkoul, M., \& Uiters, E. (2014). Overgewicht: Zijn er verschillen naar sociaal economische status?. Bilthoven: RIVM.

Schaper, L., \& Pervan, G. (2007). ICT and OTs: A model of information and communication technology acceptance and utilisation by occupational therapists. International Journal of Medical Informatics, 76, 212-221.

Schers, H., Wensing, M., Huijsmans, Z., van Tulder, M., \& Grol, R. (2001). Implementation barriers for general practice guidelines on low back pain: a qualitative study. Spine, 26(15), 348-353.

Schneider, F., Schulz, D., Pouwels, L., de Vries, H., \& van Osch, L. (2013). The use of a proactive dissemination strategy to optimize reach of an internet-delivered computer tailored lifestyle intervention. BMC Public Health, 13(1), 721.

Schneider, F., van Osch, L., Kremers, S., Schulz, D., van Adrichem, M., \& de Vries, H. (2011). Optimizing diffusion of an online computer tailored lifestyle program: a study protocol. BMC Public Health, 11(1), 480.

Schneider, F., van Osch, L., Schulz, D., Kremers, S., \& de Vries, H. (2012). The influence of user characteristics and a periodic email prompt on exposure to an internetdelivered computer-tailored lifestyle program. Journal of Medical Internet Research, 14(2), e40.

Schnoll, R., \& Zimmerman, B. (2001). Self-regulation training enhances dietary selfefficacy and dietary fiber consumption. Journal of the American Dietetic Association, 101(9), 1006-1011.

Schulz, D., Kremers, S., van Osch, L., Schneider, F., van Adrichem, M., \& de Vries, H. (2011). Testing a Dutch web-based tailored lifestyle programme among adults: a study protocol. BMC Public Health, 11(1), 108.

Schulz, D., Kremers, S., Vandelanotte, C., van Adrichem, M., Schneider, F., Candel, M., \& de Vries, H. (2014). Effects of a web-based tailored multiple-lifestyle intervention for adults: a two-year randomized controlled trial comparing sequential and simultaneous delivery modes. Journal of Medical Internet Research, 16(1).

Schulz, D., Schneider, F., de Vries, H., van Osch, L., van Nierop, P., \& Kremers, S. (2012). Program completion of a web-based tailored lifestyle intervention for adults: differences between a sequential and a simultaneous approach. Journal of Medical Internet Research, 14(2), e26. 
Schulz, D., Smit, E., Stanczyk, N., Kremers, S., de Vries, H., \& Evers, S. (2014). Economic evaluation of a web-based tailored lifestyle intervention for adults: Findings regarding cost-effectiveness and cost-utility from a randomized controlled trial. Journal of Medical Internet Research, 16(3), e91.

Schwarz, N. (1999). Self-reports: how the questions shape the answers. American Psychologist, 54(2), 93.

Shaw, B., \& Spokane, L. (2008). Examining the association between education level and physical activity changes during early old age. Journal of Aging and Health, 20(7), 767-787.

Sheeran, P., Webb, T., \& Gollwitzer, P. (2005). The interplay between goal intentions and implementation intentions. Personality \& Social Psychology Bulletin, 31(1), 8798.

Shephard, R. J. (2003). Limits to the measurement of habitual physical activity by questionnaires. British Journal of Sports Medicine, 37(3), 197-206.

Shortell, S., Bennett, C., \& Byck, G. (1998). Assessing the impact of continuous quality improvement on clinical practice: what it will take to accelerate progress. Milbank Quarterly, 76(4), 593-624.

Siliquini, R., Ceruti, M., Lovato, E., Bert, F., Bruno, S., de Vito, E., . . . La Torre, G. (2011). Surfing the internet for health information: an italian survey on use and population choices. BMC Medical Informatics and Decision Making, 11(1), 21.

Skinner, C., Strecher, V., \& Hospers, H. (1994). Physicians' recommendations for mammography: do tailored messages make a difference? American Journal of Public Health, 84(1), 43-49.

Smit, E., de Vries, H., \& Hoving, C. (2013). Determinants of practice nurses' intention to implement a new smoking cessation intervention: the importance of attitude and innovation characteristics. Journal of Advanced Nursing, 69(12), 2665-2674.

Smit, E., Evers, S., de Vries, H., \& Hoving, C. (2013). Cost-effectiveness and cost-utility of Internet-based computer tailoring for smoking cessation. Journal of Medical Internet Research, 15(3), e57.

Smit, E., Hoving, C., Cox, V., \& de Vries, H. (2012). Influence of recruitment strategy on the reach and effect of a web-based multiple tailored smoking cessation intervention among Dutch adult smokers. Health Education Research, 27(2), 191199.

Sniehotta, F., Scholzb, U., \& Schwarzerb, R. (2005). Bridging the intention-behaviour gap: Planning, self-efficacy, and action control in the adoption and maintenance of physical exercise. Psychology \& Health, 20(2), 143-160.

Soetens, K., Vandelanotte, C., de Vries, H., \& Mummery, K. (2014). Using online computer tailoring to promote physical activity: A randomized trial of text, video, and combined intervention delivery modes. Journal of Health Communication, 19(12), 1377-1392. 
Sorensen, G., \& Barbeau, E. (2004). Steps to a healthier US workforce: integrating occupational health and safety and worksite health promotion: state of the science. Washington: CDC.

Sparling, P., Franklin, B., \& Hill, J. (2013). Energy balance: the key to a unified message on diet and physical activity. Journal of Cardiopulmonary Rehabilitation and Prevention, 33(1), 12-15.

Spittaels, H., \& de Bourdeaudhuij, I. (2006). Implementation of an online tailored physical activity intervention for adults in Belgium. Health Promotion International, 21(4), 311-319.

Spittaels, H., de Bourdeaudhuij, I., Brug, J., \& Vandelanotte, C. (2007). Effectiveness of an online computer-tailored physical activity intervention in a real-life setting. Health Education Research, 22(3), 385-396.

Spittaels, H., de Bourdeaudhuij, I., \& Vandelanotte, C. (2007). Evaluation of a websitedelivered computer-tailored intervention for increasing physical activity in the general population. Preventive Medicine, 44(3), 209-217.

Springvloet, L., Lechner, L., de Vries, H., \& Oenema, A. (2015). Long-term efficacy of a web-based computer-tailored nutrition education intervention for adults including cognitive and environmental feedback: a randomized controlled trial. BMC Public Health, 15(1), 372.

Stanczyk, N., Bolman, C., Muris, J., \& de Vries, H. (2011). Study protocol of a Dutch smoking cessation e-health program. BMC Public Health, 11(1), 847.

Stanczyk, N., Bolman, C., van Adrichem, M., Candel, M., Muris, J., \& de Vries, H. (2014). Comparison of text and video computer-tailored interventions for smoking cessation: randomized controlled trial. Journal of Medical Internet Research, 16(3), e69.

Stanczyk, N., Crutzen, R., Bolman, C., Muris, J., \& de Vries, H. (2013). Influence of delivery strategy on message-processing mechanisms and future adherence to a dutch computer-tailored smoking cessation intervention. Journal of Medical Internet Research, 15(2), e28.

Stanczyk, N., Smit, E., Schulz, D., de Vries, H., Bolman, C., Muris, J., \& Evers, S. (2014). An economic evaluation of a video-and text-based computer-tailored intervention for smoking cessation: A cost-effectiveness and cost-utility analysis of a randomized controlled trial. PloS one, 9(10), e110117.

Steenhuis, I., Leeuwis, F., \& Vermeer, W. (2010). Small, medium, large or supersize: trends in food portion sizes in The Netherlands. Public Health Nutrition, 13(6), 852-857.

Steenhuis, I., \& Vermeer, W. (2009). Portion size: review and framework for interventions. International Journal of Behavioral Nutrition and Physical Activity, 6(1), 58. 
Stevens, G., Singh, G., Lu, Y., Danaei, G., Lin, J., Finucane, M., . . Cowan, M. (2012). National, regional, and global trends in adult overweight and obesity prevalences. Population Health Metrics, 10(1), 22.

Stevens, V., Obarzanek, E., Cook, N., Lee, I., Appel, L., West, D., . . . Bragg, C. (2001). Long-term weight loss and changes in blood pressure: results of the Trials of Hypertension Prevention, phase II. Annals of Internal Medicine, 134(1), 1-11.

Strecher, V., McClure, J., Alexander, G., Chakraborty, B., Nair, V., Konkel, J., . . Wiese, C. (2008). The role of engagement in a tailored web-based smoking cessation program: randomized controlled trial. Journal of Medical Internet Research, 10(5).

Stricker, M., Onland-Moret, N., Boer, J., van der Schouw, Y., Verschuren, W., May, A., . . . Beulens, J. (2013). Dietary patterns derived from principal component- and kmeans cluster analysis: Long-term association with coronary heart disease and stroke. Nutrition, Metabolism and Cardiovascular Diseases, 23(3), 250-256.

Stroebele, N., de Castro, J., Stuht, J., Catenacci, V., Wyatt, H., \& Hill, J. (2009). A smallchanges approach reduces energy intake in free-living humans. Journal of the American College of Nutrition, 28(1), 63-68.

Sutton, S. (1994). The past predicts the future: Interpreting behaviour-behaviour relationships in social psychological models of health behaviour.

Sweet, S., \& Fortier, M. (2010). Improving physical activity and dietary behaviours with single or multiple health behaviour interventions? A synthesis of meta-analyses and reviews. International Journal of Environmental Research and Public Health, 7(4), 1720-1743.

Sweller, J. (1994). Cognitive load theory, learning difficulty, and instructional design. Learning and Instruction, 4(4), 295-312.

Swift, J., \& Callahan, J. (2009). The impact of client treatment preferences on outcome: a meta-analysis. Journal of Clinical Psychology, 65(4), 368-381.

Swift, J., Callahan, J., \& Vollmer, B. (2011). Preferences. Journal of Clinical Psychology, 67(2), 155-165.

Swinburn, B., Caterson, I., Seidell, J., \& James, W. (2004). Diet, nutrition and the prevention of excess weight gain and obesity. Public Health Nutrition, 7(1), 123146.

Tabachnick, B., \& Fidell, L. (2001). Using multivariate statistics. Boston: Allyn and Bacon.

Tam, K., \& Ho, S. (2005). Web personalization as a persuasion strategy: An elaboration likelihood model perspective. Information Systems Research, 16(3), 271-291.

Tate, D., Finkelstein, E., Khavjou, O., \& Gustafson, A. (2009). Cost effectiveness of internet interventions: review and recommendations. Annals of Behavioral Medicine, 38(1), 40-45. 
The World Bank. (2015). Internet users. Retrieved 11 November, 2014 from http://data.worldbank.org/indicator/it.net.user.p2

Togo, P., Osler, M., Sørensen, T., \& Heitmann, B. (2001). Food intake patterns and body mass index in observational studies. International Journal of Obesity, 25(12), 1741-1751.

Tong, A., Sainsbury, P., \& Craig, J. (2007). Consolidated criteria for reporting qualitative research (COREQ): a 32-item checklist for interviews and focus groups. International Journal for Quality in Health Care, 19(6), 349-357.

Tremblay, M., Colley, R., Saunders, T., Healy, G., \& Owen, N. (2010). Physiological and health implications of a sedentary lifestyle. Applied Physiology, Nutrition, and Metabolism, 35(6), 725-740.

Trost, S., Owen, N., Bauman, A., Sallis, J., \& Brown, W. (2002). Correlates of adults' participation in physical activity: review and update. Medicine \& Science in Sports \& Exercise, 34(12), 1996-2001.

Tuong, W., Larsen, E., \& Armstrong, A. (2014). Videos to influence: a systematic review of effectiveness of video-based education in modifying health behaviors. Journal of Behavioral Medicine, 37(2), 218-233.

Uiters, E., \& Verweij, A. (2012). Overgewicht: Zijn er verschillen naar sociaaleconomische status?. Retrieved 3 July, 2012, from: http://www.nationaalkompas.nl/gezondheidsdeterminanten/persoonsgebonden/lichaamsgewicht/verschillensociaaleconomisch/

UNESCO Institute for Statistics. (2012). International Standard Classification of education 2011. Montreal: UNESCO Institute for Statistics.

van Assema, P., Brug, J., Ronda, G., \& Steenhuis, I. (2001). The relative validity of a short Dutch questionnaire as a means to categorize adults and adolescents to total and saturated fat intake. Journal of Human Nutrition and Dietetics, 14(5), 377-390.

van Bakel, A., \& Zantinge, E. (2014). Neemt het aantal mensen met overgewicht toe of af?. Retrieved 30 April, 2014, from http://www.nationaalkompas.nl/gezondheidsdeterminanten/persoonsgebonden/overgewicht/trend/

Van den Brink, C., \& Savelkoul, M. (2013). Gezondheidsmonitor GGD'en, CBS en RIVM. Bilthoven: RIVM.

van Deursen, A., \& van Dijk, J. (2012). Trendrapport internetgebruik 2012. Een Nederlands en Europees perspectief. Enschede: Universiteit Twente.

van Gemert-Pijnen, J., Peters, O., \& Ossebaard, H. (2013). Improving eHealth. Amsterdam: Eleven International Publishing.

van Genugten, L., van Empelen, P., Boon, B., Borsboom, G., Visscher, T., \& Oenema, A. (2012). Results from an online computer-tailored weight management intervention for overweight adults: randomized controlled trial. Journal of Medical Internet Research, 14(2), e44. 
van Genugten, L., van Empelen, P., Flink, I., \& Oenema, A. (2010). Systematic development of a self-regulation weight-management intervention for overweight adults. BMC Public Health, 10(1), 649.

van Genugten, L., van Empelen, P., \& Oenema, A. (2012). From weight management goals to action planning: identification of a logical sequence from goals to actions and underlying determinants. Journal of Human Nutrition and Dietetics, 25(4), 354364.

van Kreijl, C., Knaap, A., \& van Raaij, J. (2006). Our food, our health. Bilthoven: RIVM.

van Rossum, C., Fransen, H., Verkaik-Kloosterman, J., Buurma-Rethans, E., \& Ocké, M. (2011). Dutch National Food Consumption Survey 2007-2010. Diet of children and adults aged 7 to 69 years. Bilthoven: RIVM.

van Sluijs, E., Griffin, S., \& van Poppel, M. (2007). A cross-sectional study of awareness of physical activity: associations with personal, behavioral and psychosocial factors. International Journal of Behavioral Nutrition and Physical Activity, 4(1), 53.

van Stralen, M., de Vries, H., Bolman, C., Mudde, A., \& Lechner, L. (2010). Exploring the efficacy and moderators of two computer-tailored physical activity interventions for older adults: a randomized controlled trial. Annals of Behavioral Medicine, 39(2), 139-150.

van Stralen, M., de Vries, H., Mudde, A., Bolman, C., \& Lechner, L. (2011). The long-term efficacy of two computer-tailored physical activity interventions for older adults: Main effects and mediators. Health Psychology, 30(4), 442.

Vandelanotte, C., Duncan, M., Plotnikoff, R., \& Mummery, W. (2012). Do participants' preferences for mode of delivery (text, video, or both) influence the effectiveness of a web-based physical activity intervention? Journal of Medical Internet Research, 14(1), e37.

Vandelanotte, C., \& Mummery, W. (2011). Qualitative and quantitative research into the development and feasibility of a video-tailored physical activity intervention. International Journal of Behavioral Nutrition and Physical Activity, 8(1), 70.

Vandelanotte, C., Spathonis, K., Eakin, E., \& Owen, N. (2007). Website-delivered physical activity interventions: a review of the literature. American Journal of Preventive Medicine, 33(1), 54-64.

Verheijden, M., Jans, M., \& Hildebrandt, V. (2008). Web-based tailored lifestyle programs: exploration of the target group's interests and implications for practice. Health Promotion Practice, 9(1), 82-92.

Verheijden, M., Jans, M., Hildebrandt, V., \& Hopman-Rock, M. (2007). Rates and determinants of repeated participation in a web-based behavior change program for healthy body weight and healthy lifestyle. Journal of Medical Internet Research, 9(1). 
Verkleij, S., Adriaanse, M., Wendel-Vos, G., \& Schuit, A. (2012). Longitudinal relation between weight change and quality of life in a community-based population: a prospective cohort study. European Journal of Public Health, 285-290.

Verweij, A. (2013). Onderwijsdeelname: Indeling opleidingsniveau. Bilthoven: RIVM.

Verweij, L., Proper, K., Leffelaar, E., Weel, A., Nauta, A., Hulshof, C., \& van Mechelen, W. (2012). Barriers and facilitators to implementation of an occupational health guideline aimed at preventing weight gain among employees in the Netherlands. Journal of Occupational and Environmental Medicine, 54(8), 954-960.

Visscher, T., van Bakel, A., \& Zantinge, E. (2014). Wat zijn de mogelijke gezondheidsgevolgen van overgewicht?. Retrieved 21 October, 2014 from http://www.nationaalkompas.nl/gezondheidsdeterminanten/persoonsgebonden/ove rgewicht/wat-zijn-de-mogelijke-gezondheidsgevolgen-van-overgewicht/

Walthouwer, M., Oenema, A., Lechner, L., \& de Vries, H. (submitted). Are there differences in effects and appreciation between a video and text web-based computer-tailored obesity prevention intervention? A randomized controlled trial.

Walthouwer, M., Oenema, A., Soetens, K., Lechner, L., \& de Vries, H. (2013). Systematic development of a text-driven and a video-driven web-based computer-tailored obesity prevention intervention. BMC Public Health, 13(1), 978.

Wang, J., Schwartz, M., Luta, G., Maxwell, A., \& Mandelblatt, J. (2012). Intervention tailoring for Chinese American women: comparing the effects of two videos on knowledge, attitudes and intentions to obtain a mammogram. Health Education Research, 27(3), 523-536.

Wang, W., Worsley, A., \& Hodgson, V. (2012). Classification of main meal patterns-a latent class approach. British Journal of Nutrition, 1(1), 1-12.

Wang, Y., McPherson, K., Marsh, T., Gortmaker, S., \& Brown, M. (2011). Health and economic burden of the projected obesity trends in the USA and the UK. Lancet, 378(9793), 815-825.

Wangberg, S., Bergmo, T., \& Johnsen, J. (2008). Adherence in Internet-based interventions. Patient Preference and Adherence, 2, 57-65.

Wantland, D., Portillo, C., Holzemer, W., Slaughter, R., \& McGhee, E. (2004). The effectiveness of web-based vs. non-web-based interventions: a meta-analysis of behavioral change outcomes. Journal of Medical Internet Research, 6(4), e40.

Webb, T., Joseph, J., Yardley, L., \& Michie, S. (2010). Using the internet to promote health behavior change: a systematic review and meta-analysis of the impact of theoretical basis, use of behavior change techniques, and mode of delivery on efficacy. Journal of Medical Internet Research, 12(1), e4.

Weiner, B., Lewis, M., \& Linnan, L. (2009). Using organization theory to understand the determinants of effective implementation of worksite health promotion programs. Health Education Research, 24(2), 292-305.

Weinstein, N. (1988). The Precaution Adoption Process. Health Psychology, 7(4), 355. 
Weinstein, N., \& Lyon, J. (1999). Mindset, optimistic bias about personal risk and healthprotective behaviour. British Journal of Health Psychology, 4(4), 289-300.

Weiss, E., Galuska, D., Khan, L., Gillespie, C., \& Serdula, M. (2007). Weight regain in US adults who experienced substantial weight loss, 1999-2002. American Journal of Preventive Medicine, 33(1), 34-40.

Wendel-Vos, G. (2014a). Hoeveel mensen zijn voldoende lichamelijk actief?. Retrieved 22 October, 2014 from http://www.nationaalkompas.nl/gezondheidsdeterminanten/leef-stij1/lichamelijkeactiviteit/hoeveel-mensen-zijn-voldoende-lichamelijk-actief/

Wendel-Vos, G. (2014b). Neemt het aantal mensen met voldoende lichamelijke activiteit toe of af? $\quad$ Retrieved 22 October, 2014 from http://www.nationaalkompas.nl/gezondheids-determinanten/leefstijl/lichamelijkeactiviteit/trend/

Wendel-Vos, G., Schuit, A., Saris, W., \& Kromhout, D. (2003). Reproducibility and relative validity of the short questionnaire to assess health-enhancing physical activity. Journal of Clinical Epidemiology, 56(12), 1163-1169.

Wendel-Vos, G., \& Schuit, J. (2002). SQUASH: Short Questionnaire to Assess Health Enhancing Physical Activity. RIVM, 1-55.

Wendel-Vos, G., Droomers, M., Kremers, S., Brug, J., \& van Lenthe, F. (2007). Potential environmental determinants of physical activity in adults: a systematic review. Obesity Reviews, 8(5), 425-440.

White, I., Royston, P., \& Wood, A. (2011). Multiple imputation using chained equations: issues and guidance for practice. Statistics in Medicine, 30(4), 377-399.

WHO. (2000). Obesity: preventing and managing the global epidemic. Geneva: WHO.

WHO. (2002). The world health report. Reducing risks, promoting healthy life. Geneva: WHO.

WHO. (2003). Diet, nutrition and the prevention of chronic diseases. Report of a joint WHO/FAO expert consultation. Geneva: WHO.

WHO. (2008). Global strategy on diet, physical activity and health. Geneva: WHO.

WHO. (2013a). Obesity and overweight. Retrieved 12 March, 2013 from http://www.who.int/mediacentre/factsheets/fs311/en/

WHO. (2013b). What are the health consequences of being overweight?. Retrieved 21 October, 2014 from http://www.who.int/features/qa/49/en/

WHO. (2014a). Obesity and overweight. Retrieved 30 April, 2014 from http://www.who.int/mediacentre/factsheets/fs311/en/

WHO. (2014b). Obesity: situation and trends. Retrieved 30 April, 2014 from http://www.who.int/gho/ncd/risk_factors/obesity_text/en/

WHO. (2015a). Obesity. Retrieved 1 March, 2015 from http://www.who.int/topics/obesity/en/ 
WHO. (2015b). Obesity: situation and trends. Retrieved 21 January, 2015 from http://www.who.int/gho/ncd/risk_factors/obesity_text/en/

WHO. (2015c). Physical activity. Retrieved 21 January, 2015 from http://www.who.int/mediacentre/factsheets/fs385/en/

Whysall, Z., Haslam, C., \& Haslam, R. (2006). Implementing health and safety interventions in the workplace: An exploratory study. International Journal of Industrial Ergonomics, 36(9), 809-818.

Wieland, L., Falzon, L., Sciamanna, C., Trudeau, K., Brodney, S., Schwartz, J., \& Davidson, K. (2012). Interactive computer-based interventions for weight loss or weight maintenance in overweight or obese people. Cochrane, 8(8).

Wierenga, D., Engbers, L., van Empelen, P., Duijts, S., Hildebrandt, V., \& van Mechelen, W. (2013). What is actually measured in process evaluations for worksite health promotion programs: a systematic review. BMC Public Health, 13(1), 1190.

Williams-Piehota, P., Schneider, T., Pizarro, J., Mowad, L., \& Salovey, P. (2003). Matching health messages to information-processing styles: Need for cognition and mammography utilization. Health Communication, 15(4), 375-392.

Wilson, E., Makoul, G., Bojarski, E., Bailey, S., Waite, K., Rapp, D., . . Wolf, M. (2012). Comparative analysis of print and multimedia health materials: A review of the literature. Patient Education and Counseling, 89(1), 7-14.

Wilson, E., Park, D., Curtis, L., Cameron, K., Clayman, M., Makoul, G., . . . Wolf, M. (2010). Media and memory: the efficacy of video and print materials for promoting patient education about asthma. Patient Education and Counseling, 80(3), 393-398.

Wing, R. (2003). Behavioral interventions for obesity: recognizing our progress and future challenges. Obesity Research, 11, 3-6.

Wishart, D. (2001). ClustanGraphics 5. Edinburgh: Clustan Ltd.

Wyatt, S., Winters, K., \& Dubbert, P. (2006). Overweight and obesity: prevalence, consequences, and causes of a growing public health problem. American Journal of the Medical Sciences, 331(4), 166-174.

Zimmerman, B. (2000). Attaining self-regulation: A social cognitive perspective. In M. Boekaerts, P. Pintrich, \& M. Zeidner (Eds.), Handbook of self-regulation (pp. 1340). San Diego: Academic Press. 
APPENDICES 
Appendix 1. Overview of performance objectives, determinants, change objectives, theoretical methods, and practical applications

Table 1. Overview of performance objectives, determinants, change objectives, theoretical methods, and practical applications

\begin{tabular}{|c|c|c|c|c|}
\hline Performance objectives & Determinants & Change objectives & Theoretical methods & Practical applications \\
\hline \multirow[t]{4}{*}{$\begin{array}{l}\text { 1. Individuals decide to prevent } \\
\text { weight gain: maintain current } \\
\text { weight }(100 \mathrm{kcal}) \text { or lose a little } \\
\text { weight }(200 \mathrm{kcal})\end{array}$} & Knowledge & $\begin{array}{l}\text { Can explain what the energy balance is } \\
\text { and which efforts are required to } \\
\text { maintain weight and lose a little weight }\end{array}$ & Providing information & $\begin{array}{l}\text { Video-text intervention: an actor (i.e. expert in the field of } \\
\text { nutrition and physical activity) will give factual information } \\
\text { about the energy balance and body weight. Participants will } \\
\text { also receive basic information about the efforts that are } \\
\text { required to maintain weight and lose a little weight. } \\
\text { Text-text intervention: this information is described in text. }\end{array}$ \\
\hline & Awareness & $\begin{array}{l}\text { Can express whether own body weight } \\
\text { is healthy or not }\end{array}$ & $\begin{array}{l}\text { Consciousness raising } \\
\text { Feedback }\end{array}$ & $\begin{array}{l}\text { Video-text intervention: an actor will give feedback on } \\
\text { participants' BMI by comparing their BMI to the BMI } \\
\text { categories. Below the video message, participants' actual BMI } \\
\text { will be shown. } \\
\text { Text-text intervention: this feedback is described in text. }\end{array}$ \\
\hline & $\begin{array}{l}\text { Risk } \\
\text { perception }\end{array}$ & $\begin{array}{l}\text { Acknowledge health consequences of } \\
\text { weight gain and obesity }\end{array}$ & $\begin{array}{l}\text { Personalize risk } \\
\text { Providing information }\end{array}$ & $\begin{array}{l}\text { Video-text intervention: an actor will give feedback on } \\
\text { participants' risk perception by giving information about } \\
\text { participants' personal chances to gain weight in future as well } \\
\text { as the consequences of being overweight and obese. } \\
\text { Text-text intervention: this feedback is described in text. }\end{array}$ \\
\hline & Self-efficacy & $\begin{array}{l}\text { Can indicate for which weight goal they } \\
\text { have the highest level of confidence }\end{array}$ & Persuasion & $\begin{array}{l}\text { Video-text intervention: an actor will give information about } \\
\text { the size of the changes participants should make for } \\
\text { maintaining weight or losing a little weight. } \\
\text { Text-text intervention: this information is described in text. }\end{array}$ \\
\hline \multirow[t]{2}{*}{$\begin{array}{l}\text { 2. Individuals decide to make at } \\
\text { least one small change in dietary } \\
\text { intake and/or physical activity }\end{array}$} & Knowledge & $\begin{array}{l}\text { Can explain which small changes are } \\
\text { required in dietary intake and/or } \\
\text { physical activity }\end{array}$ & Providing information & $\begin{array}{l}\text { Video-text intervention: an actor will give factual information } \\
\text { about the small changes approach as well as the required } \\
\text { change in dietary intake and physical activity in order to } \\
\text { maintain weight and lose a little weight. } \\
\text { Text-text intervention: this information is described in text. }\end{array}$ \\
\hline & Awareness & $\begin{array}{l}\text { Can express which behavior change } \\
\text { (physical activity or dietary intake) } \\
\text { offers most possibilities for } \\
\text { improvement }\end{array}$ & $\begin{array}{l}\text { Consciousness raising } \\
\text { Feedback }\end{array}$ & $\begin{array}{l}\text { Video-text intervention: participants will fill out a } \\
\text { questionnaire about their physical activity pattern and dietary } \\
\text { intake. Based on their answers, an actor will give feedback on } \\
\text { their behaviors and indicate which behavior change would be } \\
\text { best for them. } \\
\text { Text-text intervention: this feedback is described in text. }\end{array}$ \\
\hline
\end{tabular}




\begin{tabular}{|c|c|c|c|c|}
\hline & Attitude & $\begin{array}{l}\text { Can indicate which behavior change } \\
\text { they feel most positive about }\end{array}$ & $\begin{array}{l}\text { Feedback } \\
\text { Decisional balance } \\
\text { Counter conditioning }\end{array}$ & $\begin{array}{l}\text { Participants can choose advantages and disadvantages of } \\
\text { dietary and physical activity behavior. Advantages will be } \\
\text { confirmed, while disadvantages will be countered. It will also } \\
\text { be indicated which behavior change they are most positive } \\
\text { about. }\end{array}$ \\
\hline & Self-efficacy & $\begin{array}{l}\text { Can indicate for which behavior change } \\
\text { they have the highest level of } \\
\text { confidence }\end{array}$ & $\begin{array}{l}\text { Feedback } \\
\text { Decisional balance }\end{array}$ & $\begin{array}{l}\text { Participants will answer self-efficacy questions and based on } \\
\text { their answers, it will be indicated which behavior change they } \\
\text { are most confident about. }\end{array}$ \\
\hline & $\begin{array}{l}\text { Social } \\
\text { influence }\end{array}$ & $\begin{array}{l}\text { Can indicate for which behavior change } \\
\text { they will receive most social support }\end{array}$ & $\begin{array}{l}\text { Feedback } \\
\text { Information about others' } \\
\text { approval } \\
\text { Decisional balance }\end{array}$ & $\begin{array}{l}\text { Participants will answer social influence questions and based } \\
\text { on their answers, it will be indicated for which behavior } \\
\text { change they will get most support. They will also receive } \\
\text { information about the use of social support. }\end{array}$ \\
\hline \multirow[t]{3}{*}{$\begin{array}{l}\text { 3. Individuals make plans (action } \\
\text { and preparatory) for how to carry } \\
\text { out the behavior change }\end{array}$} & Knowledge & Can explain how to make plans & Providing information & $\begin{array}{l}\text { Participants will receive information about the structure of } \\
\text { good plans and how they can identify the most personal } \\
\text { relevant 'if' and 'then'. }\end{array}$ \\
\hline & Self-efficacy & $\begin{array}{l}\text { Are able to make plans where they are } \\
\text { confident about }\end{array}$ & $\begin{array}{l}\text { Guided practice } \\
\text { Persuasion }\end{array}$ & $\begin{array}{l}\text { Participants will be shown examples of good plans and } \\
\text { informed how they can make plans step by step. }\end{array}$ \\
\hline & Skills & Apply information to make plans & $\begin{array}{l}\text { Implementation intentions } \\
\text { Providing information }\end{array}$ & $\begin{array}{l}\text { Based on the 'if-then' format, participants will make plans for } \\
\text { how to carry out the behavior change. }\end{array}$ \\
\hline \multirow[t]{2}{*}{$\begin{array}{l}\text { 4. Individuals start with the } \\
\text { chosen behavior change }\end{array}$} & Self-efficacy & $\begin{array}{l}\text { Express confidence in ability to perform } \\
\text { desired behavior }\end{array}$ & Persuasion & $\begin{array}{l}\text { The plans and goals will be summarized and participants will } \\
\text { be explained that by using their plans they are capable of } \\
\text { performing the desired behavior. }\end{array}$ \\
\hline & Skills & Are able to perform the desired behavior & Providing information & $\begin{array}{l}\text { Video-text intervention: an actor will give information about } \\
\text { how to apply action and preparatory plans in practice. } \\
\text { Text-text intervention: this information is described in text. }\end{array}$ \\
\hline \multirow[t]{2}{*}{$\begin{array}{l}\text { 5. Individuals monitor their } \\
\text { behavior and weight }\end{array}$} & Skills & $\begin{array}{l}\text { Are able to monitor behavior change } \\
\text { and compare it with goal }\end{array}$ & Self-reevaluation & $\begin{array}{l}\text { Video-text intervention: an actor will instruct participants to } \\
\text { compare their behavior with the desired behavior as specified } \\
\text { in their plans. } \\
\text { Text-text intervention: this information is described in text. }\end{array}$ \\
\hline & Self-efficacy & $\begin{array}{l}\text { Have confidence to deal with deviations } \\
\text { from the desired behavior }\end{array}$ & Persuasion & $\begin{array}{l}\text { Video-text intervention: an actor will inform participants how } \\
\text { they can monitor their behavior and how to take action when } \\
\text { the desired behavior is not performed adequately. } \\
\text { Text-text intervention: this information is described in text. }\end{array}$ \\
\hline $\begin{array}{l}\text { 6. Individuals make coping plans } \\
\text { how to deal with difficulties } \\
\text { during the behavior change }\end{array}$ & Knowledge & $\begin{array}{l}\text { Can explain how to deal with difficult } \\
\text { situations }\end{array}$ & Providing information & $\begin{array}{l}\text { Participants will receive information about how to deal with } \\
\text { expected or encountered difficult situations during the } \\
\text { behavior change. }\end{array}$ \\
\hline
\end{tabular}




\begin{tabular}{|c|c|c|c|c|}
\hline & Self-efficacy & $\begin{array}{l}\text { Are able to make coping plans where } \\
\text { they are confident about }\end{array}$ & $\begin{array}{l}\text { Guided practice } \\
\text { Modeling }\end{array}$ & $\begin{array}{l}\text { Participants will be shown examples of suitable coping plans } \\
\text { and informed how they can make coping plans step by step. } \\
\text { They will also receive examples of how other people (i.e. } \\
\text { actors) deal with difficult situations during the behavior } \\
\text { change (narratives). }\end{array}$ \\
\hline & Skills & Apply information to make coping plans & $\begin{array}{l}\text { Implementation intentions } \\
\text { Providing information }\end{array}$ & $\begin{array}{l}\text { Based on the 'if-then' format, participants will make coping } \\
\text { plans for how to deal with difficult situations. }\end{array}$ \\
\hline \multirow[t]{2}{*}{$\begin{array}{l}\text { 7. Individuals evaluate their } \\
\text { behavior change }\end{array}$} & Awareness & $\begin{array}{l}\text { Recognize when behavior change is } \\
\text { (un)successful }\end{array}$ & $\begin{array}{l}\text { Consciousness raising } \\
\text { Feedback }\end{array}$ & $\begin{array}{l}\text { Video-text intervention: an actor will indicate whether or not } \\
\text { participants have achieved their behavior change goal by } \\
\text { comparing participants' current behavior with their behavior } \\
\text { at the start of the program. Below this video message, a table } \\
\text { will be shown that provides an overview of participants' } \\
\text { current and previous behavior. } \\
\text { Text-text intervention: this feedback is described in text and } \\
\text { includes the same table. }\end{array}$ \\
\hline & Self-efficacy & $\begin{array}{l}\text { Have confidence about how to evaluate } \\
\text { behavior change }\end{array}$ & $\begin{array}{l}\text { Reinforcement } \\
\text { External attribution } \\
\text { Modeling }\end{array}$ & $\begin{array}{l}\text { Video-text intervention: an actor will reinforce participants } \\
\text { who achieved their behavior change goal. Failure will be } \\
\text { attributed to external factors. Multiple actors will further also } \\
\text { act in role model stories (narratives) by serving as examples } \\
\text { of how other people deal with (un)successful behavior } \\
\text { changes. } \\
\text { Text-text intervention: this feedback and the narratives are } \\
\text { described in text. }\end{array}$ \\
\hline \multirow[t]{2}{*}{$\begin{array}{l}\text { 8. Individuals evaluate their } \\
\text { weight change }\end{array}$} & Awareness & $\begin{array}{l}\text { Recognize when weight change is } \\
\text { (un)successful }\end{array}$ & $\begin{array}{l}\text { Consciousness raising } \\
\text { Feedback }\end{array}$ & $\begin{array}{l}\text { Video-text intervention: an actor will indicate whether or not } \\
\text { participants achieved their weight goal by comparing } \\
\text { participants' current weight with their weight at the start of } \\
\text { the program. Below this video message, a table will be shown } \\
\text { that provides an overview of participants' weight during the } \\
\text { program. } \\
\text { Text-text intervention: this feedback is described in text and } \\
\text { includes the same table. }\end{array}$ \\
\hline & Self-efficacy & $\begin{array}{l}\text { Have confidence about how to evaluate } \\
\text { weight change }\end{array}$ & $\begin{array}{l}\text { Reinforcement } \\
\text { External attribution } \\
\text { Modeling }\end{array}$ & $\begin{array}{l}\text { Video-text intervention: an actor will reinforce participants } \\
\text { who have achieved their weight goal. Failure will be } \\
\text { attributed to external factors. Multiple actors will further also } \\
\text { act in role model stories (narratives) by serving as examples } \\
\text { of how other people deal with (un)successful weight changes. } \\
\text { Text-text intervention: this feedback and the narratives are } \\
\text { described in text. }\end{array}$ \\
\hline
\end{tabular}




\begin{tabular}{|c|c|c|c|c|}
\hline \multirow{2}{*}{$\begin{array}{l}\text { 9. Individuals decide to maintain } \\
\text { the behavior change or to make a } \\
\text { new behavior change (and go back } \\
\text { to PO 2) }\end{array}$} & Awareness & $\begin{array}{l}\text { Are aware of their weight and behavior } \\
\text { change progress }\end{array}$ & $\begin{array}{l}\text { Consciousness raising } \\
\text { Feedback }\end{array}$ & $\begin{array}{l}\text { Video-text intervention: an actor will indicate whether or not } \\
\text { participants achieved their weight and behavior change goals. } \\
\text { Text-text intervention: this information is described in text. }\end{array}$ \\
\hline & Self-efficacy & $\begin{array}{l}\text { Have confidence in weight and behavior } \\
\text { change goals }\end{array}$ & $\begin{array}{l}\text { Persuasion } \\
\text { Reinforcement } \\
\text { External attribution }\end{array}$ & $\begin{array}{l}\text { Video-text intervention: an actor will indicate that participants } \\
\text { who achieved their weight goal successfully can try to make a } \\
\text { more challenging behavior change. Participants who failed to } \\
\text { achieve their weight goal will be asked if they want to make } \\
\text { an easier behavior change. } \\
\text { Text-text intervention: this information is described in text. }\end{array}$ \\
\hline \multirow[t]{4}{*}{$\begin{array}{l}\text { 10. Individuals maintain the } \\
\text { behavior change }\end{array}$} & Knowledge & $\begin{array}{l}\text { Can tell how to maintain behavior } \\
\text { change on long-term }\end{array}$ & Providing information & $\begin{array}{l}\text { Video-text intervention: an actor will give information about } \\
\text { how participants can maintain behavior changes on the long- } \\
\text { term. } \\
\text { Text-text intervention: this information is described in text. }\end{array}$ \\
\hline & Skills & $\begin{array}{l}\text { Apply information to set a long-term } \\
\text { weight goal }\end{array}$ & Providing information & $\begin{array}{l}\text { Participants will set a long-term weight goal by choosing a } \\
\text { time period and a desired future weight. }\end{array}$ \\
\hline & Self-efficacy & $\begin{array}{l}\text { Have confidence to maintain behavior } \\
\text { change }\end{array}$ & $\begin{array}{l}\text { Providing information } \\
\text { Persuasion }\end{array}$ & $\begin{array}{l}\text { Video-text intervention: an actor will explain that participants } \\
\text { can maintain behavior change(s) by means of the skills they } \\
\text { have acquired during the intervention. } \\
\text { Text-text intervention: this information is described in text. }\end{array}$ \\
\hline & Intention & $\begin{array}{l}\text { Are motivated to maintain behavior } \\
\text { change }\end{array}$ & Arguments & $\begin{array}{l}\text { Video-text intervention: an actor will give factual information } \\
\text { about the advantages of maintaining behavior changes on the } \\
\text { long-term. } \\
\text { Text-text intervention: this information is described in text. }\end{array}$ \\
\hline
\end{tabular}




\section{Appendix 2. Example of the text intervention}

\section{Welcome to Weight in the Balance!}

Dear participant,

Welcome to the program Weight in the Balance! Before starting with the program, you can first read some information about how the program works.

\section{What is the program Weight in the Balance?}

Weight in the Balance provides you with help and support to control your weight. Controlling your weight refers to preventing weight gain or achieving modest weight loss. This can already be achieved by making small changes in your diet and physical activity behavior.

Weight in the Balance does not focus on losing a lot of weight. To lose a lot of weight it is necessary to follow a strict diet and an intensive sport schedule. Most people experience this as very difficult. Therefore, this program focuses on small changes in dietary intake and physical activity that can help you in maintaining your weight or achieving modest weight loss.

\section{Next}

On the following pages you can read more about the program. By clicking on 'next' you can go through the program.

\section{Appendix 3. Example of the video intervention}

Welcome to Weight in the Balance!

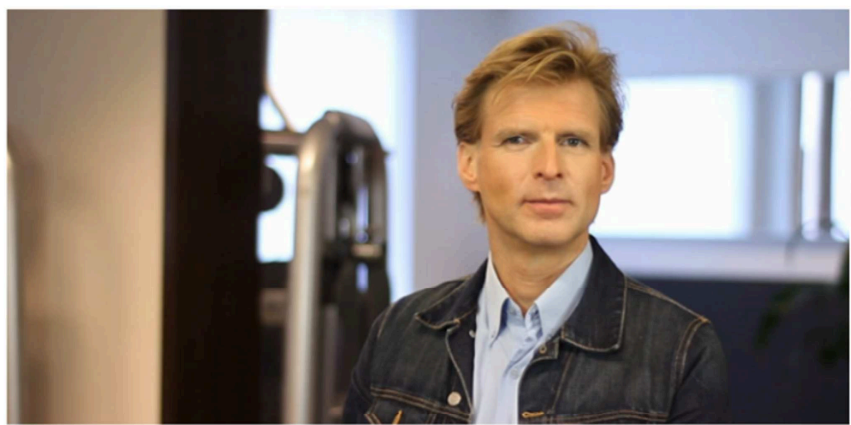




\section{SUMMARY}


Overweight and obese people have an increased risk of developing health problems such as cardiovascular diseases, type 2 diabetes mellitus, mental disorders, and different types of cancer. Currently, about 1.4 billion adults are overweight and over 600 million adults are obese. Overweight and obesity rates are further significantly higher among people with a low educational level. Hence, there is a need for weight gain prevention interventions that are effective and available for adults with a low educational level in particular. These interventions should aim to achieve a balance between energy intake (via diet) and energy expenditure (via physical activity). Prevention of weight gain can be achieved with small changes in dietary intake or physical activity of approximately 100 kilocalories per day, whereas daily changes of 200 kilocalories can result in modest weight loss (i.e. about 5 kilograms per year).

It has been suggested that web-based computer-tailored interventions are a promising approach for preventing weight gain since these interventions can provide highly individualized information to large numbers of people against relatively low costs. However, much previous research on web-based computer-tailored weight management interventions has reported problems with their use and efficacy, which limits their potential impact. It has been suggested that the use of videos to deliver intervention content may improve the use and efficacy of these interventions among people with a low educational level in particular. Videos are interactive and reduce the cognitive effort needed to process information. The main aim of this dissertation was to examine the appreciation, use, and efficacy of a video and text version of a web-based computer-tailored obesity prevention intervention among lower and higher educated Dutch adults with a healthy weight or limited overweight.

The dissertation was divided into two parts. The aim of the first part, which consisted of two exploratory studies, was to examine how eating in moderation should be stimulated in interventions. The second part of the dissertation consisted of four studies that aimed to provide insight into the development process as well as the effect and process evaluation of the video and text version of the web-based computer-tailored obesity prevention intervention.

Chapter 1 provides a general introduction of the background and theoretical underpinnings of the different studies presented in this dissertation, including an overview of the different research questions.

Chapter 2 describes a longitudinal study that aimed to examine the psychosocial determinants of eating in moderation and the possible moderating role of awareness at 6month follow-up ( $\mathrm{N}=379)$. Compared to unaware participants $(48.1 \%)$, participants who were aware of their dietary behavior had a significantly lower average daily energy intake. Among the aware participants, eating in moderation was predicted by demographic variables and risk perception, attitude, subjective norm, modelling, and intention. Among the unaware participants, besides several demographics, only risk perception and selfefficacy were significantly associated with eating in moderation. Based on the findings of 
this study, it can be concluded that interventions aimed at promoting eating in moderation should first focus on improving people's awareness of their dietary behavior before targeting motivational factors.

Chapter 3 describes the results of a longitudinal study that aimed to (1) identify clusters of dietary patterns among Dutch adults, (2) test the reproducibility of these clusters and stability of cluster membership over time, and (3) identify sociodemographic predictors of cluster membership and cluster transition. At both baseline $(\mathrm{N}=483)$ and 6-month follow-up ( $\mathrm{N}=379)$, a healthy, moderately healthy, and unhealthy dietary pattern were identified. Only gender and living situation were significantly associated with cluster membership and cluster transition. Further, only 251 (66.2\%) participants remained in the same cluster, while $45(11.9 \%)$ participants changed to an unhealthier cluster and 83 (21.9\%) participants shifted to a healthier cluster. Since one-third of the participants changed from cluster of dietary pattern over time and only few factors predicted cluster membership and cluster transition, it can be concluded that it may not be efficient to target interventions at clusters of dietary patterns.

Chapter 4 describes the systematic development process of the video and text version of the web-based computer-tailored obesity prevention intervention for Dutch adults by means of the Intervention Mapping protocol. Both versions had exactly the same educational content, but in the video version the core messages were provided via tailored videos in addition to text-based general information. The aim of the intervention was to achieve small changes in dietary intake and physical activity in order to prevent obesity (i.e. achieve weight maintenance or modest weight loss). The intervention was framed around self-regulation theories and the I-Change Model and consisted of six sessions. In the first two sessions, participants had to set weight and behavioral change goals and form plans for specific actions to achieve the desired goals. In the remaining four sessions, participants' evaluated their progress toward achievement of their goals and they had the opportunity to make coping plans for difficult situations.

Chapter 5 describes the results of a randomized controlled trial (RCT) that aimed to evaluate the effects of the video and text version of the intervention in comparison to a waiting list control condition on self-reported dietary intake, physical activity level, and BMI among Dutch adults, including possible moderation of educational level. Another study aim was to examine differences in appreciation between the video and text version, including possible differences per educational level as well. At 6 month follow-up $(\mathrm{N}=1,015)$, the video version had resulted in lower BMI and lower average daily energy intake from energy-dense food products, while the text version only had an effect on the latter one. For physical activity no intervention effects were found for both the video and text condition in comparison to the control condition. In addition, no significant interaction effects of educational level were found on any of the outcome variables. These results were found with both the multiple imputation as completers only dataset. Furthermore, the video version was rated significantly better than the text version on feelings of relatedness, 
usefulness, and grade given to the intervention. Based on these results it can be concluded that the video version of the web-based computer-tailored obesity prevention intervention was the most effective intervention and appreciated best, regardless of participants' educational level.

Chapter 6 describes the results of a RCT that aimed to examine if there were differences in intervention use between the video and text version as well as between allocation to a matching and mismatching intervention delivery format. Another study aim was to assess the possible moderating role of intervention use and (mis)matching delivery format in the intervention effects on self-reported BMI, physical activity, and energy intake. The final study aim was to identify the predictors of intervention use. The results showed that use of both the video and text version declined rapidly over time and that there were no significant differences in use between the two versions, regardless of participants' educational level. The average number of completed sessions was significantly higher among participants who were allocated to an intervention condition that matched their preferred intervention delivery format compared to participants with a mismatched delivery format. Yet, the intervention effects were not moderated by intervention use and allocation to a (mis)matching intervention delivery format. These results were found with both the multiple imputation as completers only dataset. Moreover, participants with a high BMI and those who felt highly related to the intervention were more likely to use the intervention more frequently. Based on these findings it can be concluded that the use of web-based computer-tailored interventions needs improvement and that this can possibly be achieved by delivering intervention content via participants' preferred intervention delivery format and creating feelings of relatedness.

Chapter 7 describes a qualitative study that aimed to identify barriers and facilitators to the adoption and particularly the implementation of the web-based computertailored obesity prevention intervention by occupational health centers. Participants were directors of Dutch occupational health centers who had decided to either participate $(\mathrm{N}=8)$ or not participate $(\mathrm{N}=12)$ in the RCT regarding the evaluation of the intervention. A semistructured telephone interview was conducted with each participant and the results were analyzed by means of a directed content analysis approach. There were important differences in opinions between participants who had adopted and implemented the intervention and those who had not, particularly on barriers and facilitators related to the intervention and the personal beliefs of the implementer. The relative advantages of the intervention and interviewees' personal attitudinal and self-efficacy beliefs were considered to be most important. Regarding the organization, the possibilities to increase profits and integrate the intervention within the organization were considered to be important facilitators. Based on the findings of this study, it can be concluded that strategies to facilitate the implementation of web-based computer-tailored obesity prevention interventions by occupational health centers should be tailored to implementers' unique perceptions toward implementing these interventions. 
Finally, chapter 8 provides a general discussion of the results of all the studies presented in this dissertation, including an overview of the methodological considerations and recommendations for practice and future research. Preliminary analyses with the 12month follow-up data indicate that the effects of the video version on BMI are maintained, whereas the text version also has an effect on this outcome measure. In conclusion, the results indicate that the video version of the web-based computer-tailored intervention seems most suitable for implementation in practice since it achieved better short-term effects, higher effect sizes, and was appreciated better than the text version. Yet, future research is needed in order to examine how the (video version of the) web-based computertailored obesity prevention intervention can be further optimized. 


\section{SAMENVATTING}


Wereldwijd zijn er op dit moment ongeveer 1,4 miljard volwassenen met overgewicht en meer dan 600 miljoen volwassenen met zwaar overgewicht (obesitas). Mensen met overgewicht en obesitas hebben een verhoogde kans op het krijgen van gezondheidsproblemen zoals hart- en vaatziekten, suikerziekte, psychische stoornissen en verschillende soorten kanker. Gezien de ernst en omvang van deze problemen is er behoefte aan gezondheidsbevorderende programma's die overgewicht en obesitas op een effectieve manier kunnen voorkomen en veel mensen kunnen bereiken. Overgewicht en obesitas kunnen worden voorkomen door ervoor te zorgen dat het gewicht van mensen gezond blijft en niet steeds verder toeneemt. Het voorkomen van gewichtstoename kan al worden bereikt met kleine veranderingen in eten of bewegen van 100 kilocalorieën per dag. Dit is gelijk aan ongeveer 15 minuten wandelen of het drinken van een glas water in plaats van een glas cola. Met het dubbele, dagelijkse veranderingen van 200 kilocalorieën, kan zelfs een kleine gewichtsafname van ongeveer vijf kilogram per jaar worden bereikt.

Advies-op-maat internetprogramma's kunnen mogelijk bijdragen aan het voorkomen van overgewicht en obesitas op een kosteneffectieve manier. In deze programma's zijn de adviezen en informatie helemaal afgestemd op de persoonlijke kenmerken van de gebruiker. Doordat deze programma's via het internet kunnen worden verspreid, kunnen grote groepen mensen worden bereikt tegen relatief lage kosten. Echter, eerder onderzoek heeft aangetoond dat de effecten van deze programma's over het algemeen klein tot middelgroot zijn. Daarnaast worden deze programma's meestal maar kortdurend gebruikt, waardoor mensen vaak onvoldoende inzicht en vaardigheden mee krijgen om hun gedrag succesvol en blijvend te veranderen. Het is daarom belangrijk om te onderzoeken hoe het gebruik en de effectiviteit van advies-op-maat internetprogramma's kan worden verbeterd. Het aanbieden van informatie en adviezen in de vorm van video's (beeldmateriaal) is hier mogelijk een oplossing voor. Video's zijn namelijk interactiever dan tekst en verminderen de inspanning die vereist is om informatie te verwerken. Video's zijn daarom mogelijk in het bijzonder geschikt voor mensen met een laag opleidingsniveau. Deze mensen vormen een belangrijke doelgroep aangezien ze vaker overgewicht en obesitas hebben.

Dit proefschrift beschrijft het onderzoek naar de ontwikkeling en evaluatie van een video- en tekstversie van een advies-op-maat internetprogramma. Het doel van dit programma was het bereiken van kleine veranderingen in energie-inname en lichamelijke activiteit om obesitas te voorkomen (bereiken van gewichtsbehoud of kleine gewichtsafname) onder Nederlandse volwassenen.

Het proefschrift bestaat uit twee onderdelen. Het eerste onderdeel bevat twee verkennende onderzoeken, die als doel hadden meer inzicht te krijgen in hoe een gezond eetpatroon gestimuleerd kan worden in obesitaspreventieprogramma's. Het tweede onderdeel van dit proefschrift bestaat uit vier onderzoeken en had als doel inzicht te geven in het ontwikkelingsproces en de effect- en procesevaluatie van de video- en tekstversie van het advies-op-maat internetprogramma. 
Hoofdstuk 1 bestaat uit achtergrondinformatie en onderbouwing van het onderzoek dat is beschreven in dit proefschrift, inclusief een overzicht van de verschillende onderzoeksvragen.

Hoofdstuk 2 beschrijft een longitudinaal onderzoek $(\mathrm{N}=379)$ dat als doel had het vaststellen of er een verschil was in redenen (determinanten) om met mate te eten tussen mensen die terecht dachten dat ze met mate aten (mensen die bewust waren van hun gedrag) en mensen die dachten dat ze met mate aten, maar waarbij dat niet zo bleek te zijn (onbewust van eigen gedrag). De onbewuste deelnemers $(48,1 \%)$ hadden vergeleken met de bewuste deelnemers een hogere gemiddelde dagelijkse energie-inname. De determinanten van met mate eten bij bewuste deelnemers waren verscheidene demografische kenmerken, risicoperceptie, attitude, sociale norm, modelling en intentie. Onder de onbewuste deelnemers werden enkel een aantal demografische kenmerken en risicoperceptie en eigeneffectiviteit geïdentificeerd als determinanten van met mate eten. Op basis van deze bevindingen kan worden geconcludeerd dat gezondheidsbevorderende programma's mensen eerst bewust moeten maken van hun eigen gedrag alvorens ze te kunnen motiveren om met mate te gaan eten.

Hoofdstuk 3 beschrijft de resultaten van een longitudinaal onderzoek dat drie doelen had: (1) het identificeren van eetpatronen, (2) het testen van de stabiliteit van eetpatronen, (3) het identificeren van determinanten van eetpatronen en verandering hiervan. Op baseline $(\mathrm{N}=483)$ en zes maanden later $(\mathrm{N}=379)$ werden er drie eetpatronen geïdentificeerd: een gezond, matig gezond en ongezond eetpatroon. Enkel geslacht en woonsituatie waren significante determinanten van eetpatronen en verandering hiervan. Verder hadden slechts $251(66,2 \%)$ deelnemers een stabiel eetpatroon. Na zes maanden hadden $45(11,9 \%)$ deelnemers een ongezonder eetpatroon en $83(21,9 \%)$ een gezonder eetpatroon. Aangezien eetpatronen niet per definitie stabiel zijn en verandering van eetpatroon moeilijk is te voorspellen, kan er worden geconcludeerd dat het waarschijnlijk niet effectief zal zijn om programma's te richten op eetpatronen. Het is wellicht beter om programma's te richten op losse voedingsproducten en mensen hierover zo persoonlijk mogelijk advies te geven.

Hoofdstuk 4 beschrijft het systematische ontwikkelingsproces van de video- en tekstversie van het advies-op-maat internetprogramma. Beide versies hadden exact dezelfde inhoud, maar in de videoversie werd ongeveer $75 \%$ van de informatie en adviezen gegeven aan de hand van video's. De overige $25 \%$ werd gegeven aan de hand van tekst. De tekstversie bestond enkel uit tekst. Het doel van het programma was het stimuleren van kleine veranderingen in energie-inname en beweging om gewichtsbehoud of een kleine gewichtsafname te realiseren bij Nederlandse volwassenen. Het programma was gebaseerd op zelfregulatietheorieën en het I-Change Model en bestond uit zes wekelijkse sessies. In de eerste twee sessies konden deelnemers een gewichts- en gedragsdoel kiezen en plannen hoe ze deze doelen wilden bereiken. In de resterende vier sessies konden deelnemers hun 
vooruitgang evalueren en hadden ze de mogelijkheid om plannen te maken voor het omgaan met barrières.

Hoofdstuk 5 beschrijft de resultaten van een gerandomiseerd gecontroleerd experiment (RCT) naar de effecten van de video- en tekstversie op energie-inname, beweging en BMI en de verschillen in waardering tussen de twee versies. Na zes maanden had de videoversie $(\mathrm{N}=465)$ geresulteerd in een lagere BMI en lagere energie-inname in vergelijking met de deelnemers van de controle groep $(\mathrm{N}=463)$. De tekstversie $(\mathrm{N}=491)$ had enkel geresulteerd in een lagere energie-inname. Zowel de video- als de tekstversie had geen effecten op beweging. Daarnaast werden er ook geen verschillen in effecten gevonden naar opleidingsniveau. De videoversie werd beter gewaardeerd dan de tekstversie. De videoversie scoorde hoger op gevoelens van betrokkenheid bij het programma, bruikbaarheid van de informatie en er werd een hoger waarderingscijfer toegekend. Er werden geen verschillen in waardering per opleidingsniveau gevonden. Op basis van deze resultaten kan worden geconcludeerd dat de videoversie het meest effectief is en het beste gewaardeerd wordt, ongeacht mensen hun opleidingsniveau.

Hoofdstuk 6 beschrijft de resultaten van dezelfde RCT als in hoofdstuk 5. Het eerste doel van dit onderzoek was het vaststellen van verschillen in gebruik tussen de video- en tekstversie. Een ander doel was te onderzoeken of gebruik van het programma hoger was bij deelnemers die een versie van het programma hadden gebruik die paste bij hun voorkeur. Het derde doel was te onderzoeken of de effecten van het programma beter waren bij deelnemers die het programma meer gebruikt hadden en een versie gebruikt hadden die paste bij hun voorkeur. Het laatste onderzoeksdoel was het identificeren van determinanten van gebruik. De resultaten toonden aan dat het gebruik van zowel de videoals tekstversie snel afnam per sessie. Er waren geen verschillen in gebruik tussen de videoen tekstversie, ongeacht het opleidingsniveau van de deelnemers. Echter, deelnemers die een versie van het programma hadden gebruikt die paste bij hun voorkeur hadden het programma vaker gebruikt dan de deelnemers met een niet-passende voorkeur. Het programma was verder niet effectiever wanneer het vaker werd gebruikt of wanneer deelnemers een versie hadden gevolgd die paste bij hun voorkeur. Tot slot, de determinanten van hoger gebruik van het programma waren het hebben van een hoge BMI en een hoge score op gevoelens van betrokkenheid bij het programma. Op basis van het lage gebruik van zowel de video- en tekstversie kan er geconcludeerd worden dat er verder onderzoek nodig is om te onderzoeken hoe het gebruik van het programma verbeterd kan worden. De resultaten tonen aan dat een hoger gebruik mogelijk kan worden bewerkstelligd door deelnemers een versie van het programma te laten gebruiken die past bij hun voorkeur en het creëren van gevoelens van betrokkenheid bij het programma.

Hoofdstuk 7 beschrijft een kwalitatief onderzoek met als doel het identificeren van factoren die de adoptie en in het bijzonder de implementatie van het advies-op-maat internetprogramma door arbodiensten kunnen belemmeren en stimuleren. Deelnemers waren directeuren van Nederlandse arbodiensten die besloten hadden om deel te nemen aan 
de RCT en bereid waren ons programma te implementeren $(\mathrm{N}=8)$ evenals directeuren die dit hadden geweigerd $(\mathrm{N}=12)$. Met elke deelnemer werd een telefonisch interview gehouden. De resultaten toonden aan dat er grote verschillen waren in opvattingen over factoren die de implementatie kunnen belemmeren en stimuleren tussen deelnemers die het programma hadden geïmplementeerd en degenen die dit hadden geweigerd. De deelnemers verschilden vooral in opvatting op het gebied van factoren gerelateerd aan het programma en hun persoonlijke overtuigingen. De voordelen van het programma en de attitude en eigeneffectiviteit van deelnemers bleken een belangrijke invloed te hebben op de implementatie. De mogelijkheid om winst te vergroten en het programma te integreren binnen het bedrijf waren belangrijke stimulerende factoren. Op basis van dit onderzoek kan geconcludeerd worden dat directeuren van arbodiensten verschillende opvattingen kunnen hebben over factoren die de implementatie van advies-op-maat internetprogramma's kunnen belemmeren en stimuleren. Het is daarom belangrijk om implementatiestrategieën te richten op directeuren hun unieke opvatting.

Tot slot, hoofdstuk 8 bestaat uit een discussie van de resultaten van alle onderzoeken in dit proefschrift. Daarnaast bevat dit hoofdstuk ook een overzicht van methodologische overwegingen en aanbevelingen voor de praktijk en toekomstig onderzoek. In dit hoofdstuk worden ook de effecten van het programma na twaalf maanden besproken. Hieruit blijkt dat het effect van de videoversie op BMI gehandhaafd blijft, terwijl de tekstversie dan ook een effect op BMI heeft. Samenvattend kan er geconcludeerd worden dat de videoversie van het advies-op-maat internetprogramma de voorkeur verdient boven de tekstversie aangezien de videoversie betere korte termijn effecten had, grotere effecten had en beter werd gewaardeerd. Toekomstig onderzoek is nodig om vast te stellen hoe de effecten van de videoversie kunnen worden geoptimaliseerd en hoe de implementatie van dit programma in de praktijk het beste kan worden bevorderd. 


\section{CURRICULUM VITAE}




\section{Biography}

Michel Walthouwer was born on 14 July 1987 in Maastricht, the Netherlands. After completing secondary school (Atheneum) in 2005 at Stella Maris College Meerssen, he started studying Health Sciences at Maastricht University. His specializations were Health Education (major) and Mental Health Science (minor). During his study, Michel started working at MAASTRO Clinic as a research assistant in 2009. After finishing his bachelor, he graduated as a master of Health Education and Promotion with distinction at Maastricht University in 2011. He received an award for belonging to the top 3\% students of Maastricht University during the academic year 2010-2011 (master). Immediately after finishing his master, Michel started working as a $\mathrm{PhD}$ student at the department of Health Promotion at Maastricht University to conduct the research presented in this dissertation. He has presented his research at different national and international conferences. Moreover, Michel also developed a web-based physical activity intervention for the University of Québec in Trois-Rivières, Canada. Since August 2015, Michel is working as statistical researcher at Statistics Netherlands.

\section{Publications}

d.d. 01-09-2015

\section{Publications presented in this dissertation}

Walthouwer, M., Oenema, A., Candel, M., Lechner, L., \& de Vries, H. (2015). Eating in moderation and the essential role of awareness: a Dutch longitudinal study identifying psychosocial predictors. Appetite, 87, 152-159.

Walthouwer, M., Oenema, A., Soetens, K., Lechner, L., \& de Vries, H. (2014). Are clusters of dietary patterns and cluster membership stable over time? Results of a longitudinal cluster analysis study. Appetite, 82, 154-159.

Walthouwer, M., Oenema, A., Soetens, K., Lechner, L., \& de Vries, H. (2013). Systematic development of a text-driven and a video-driven web-based computer-tailored obesity prevention intervention. BMC Public Health, 13, 978.

Walthouwer, M., Oenema, A., Lechner, L., \& de Vries, H. (accepted). Are there differences in effects and appreciation between a video and text web-based computer-tailored obesity prevention intervention? A randomized controlled trial. Journal of Medical Internet Research.

Walthouwer, M., Oenema, A., Lechner, L., \& de Vries, H. (accepted). Use and effectiveness of a video- and text-driven web-based computer-tailored intervention: does delivery format matter? Journal of Medical Internet Research.

Walthouwer, M., Oenema, A., Soetens, K., Lechner, L., \& de Vries, H. (under review). Implementation of web-based interventions by Dutch occupational health centers. 


\section{Other publications}

Walthouwer, M., Olberding, M., Hoving, C., Op de Coul, M., Janssen, E., van Kann, D., Lechner, L., de Vries, H., \& van Osch, L. (2012). Kennis van kankersymptomen en de rol van psychosociale factoren bij tijdig medisch hulpzoekgedrag. Tijdschrift voor Gezondheidswetenschappen, 1, 36-44. 


\section{DANKWOORD}


Dit proefschrift zou niet tot stand zijn gekomen zonder de bijdrage van velen. Ik wil iedereen hiervoor van harte bedanken, maar een aantal mensen wil ik in het bijzonder noemen.

Hein, allereerst wil ik je bedanken voor de kans die je me gaf om te kunnen promoveren. Ook wil ik je bedanken voor je vertrouwen in mij en de vrijheid die ik kreeg tijdens mijn ontwikkeling als onderzoeker. Je wetenschappelijke expertise en enorme creativiteit zijn voor mij erg waardevol geweest. Daarnaast wist je met je praktische en af en toe humoristische zienswijze mijn artikelen naar een hoger niveau te tillen. Jouw ideeën, creativiteit en enorme enthousiasme hebben me absoluut geïnspireerd.

Lilian, ook jou wil ik bedanken voor de kans die je me gaf om te kunnen promoveren. Je nuchtere en kritische blik hebben enorm stimulerend gewerkt en geholpen om belangrijke knopen door te hakken. Hoewel je iets meer op afstand betrokken was, hield je het overzicht en wist je altijd precies waar ik mee bezig was. Ik heb met heel veel plezier met jou samengewerkt.

Anke, je bent voor mij van hele grote waarde geweest bij de totstandkoming van dit proefschrift. Ik wil je heel erg bedanken voor je betrokkenheid en tijd om mijn ideeën en artikelen van opbouwende feedback te voorzien. Maar vooral ook bedankt voor je luisterend oor en je vele adviezen. Ik heb ontzettend veel van je geleerd en het was erg prettig dat je altijd voor me klaar stond. Je was een bijzonder fijne begeleider en collega.

Collega's van de vakgroep Gezondheidsbevordering, bedankt voor de prettige en leerzame tijd die ik hier samen met jullie heb gehad. Naast een prettige inhoudelijke samenwerking zorgden jullie ook voor voldoende gezellige momenten.

Vrienden en familie, het is onmogelijk om jullie allemaal bij naam te bedanken voor jullie vriendschap, onvoorwaardelijke steun en oprechte interesse in mijn onderzoek tijdens de afgelopen jaren. Ik ben jullie daar erg dankbaar voor.

Manon, tot slot wil ik jou bedanken voor je onvoorwaardelijke steun en geduld. Dankzij jou is mijn doorzettingsvermogen enorm gegroeid en uiteindelijk dit proefschrift tot stand gekomen. Jij compenseerde de stressvolle periodes en stimuleerde mij om het beste uit mezelf te halen. Dank dat je me altijd laat voelen dat je trots op me bent!

\section{Michel}


